\title{
The influence of the family environment on adaptive functioning in the classroom: A longitudinal study of children with developmental disabilities
}

Author: Miriam Heyman

Persistent link: http://hdl.handle.net/2345/bc-ir:104136

This work is posted on eScholarship@BC, Boston College University Libraries.

Boston College Electronic Thesis or Dissertation, 2015

Copyright is held by the author, with all rights reserved, unless otherwise noted. 


\section{BOSTON COLLEGE Lynch School of Education}

Department of Counseling, Developmental, and Educational Psychology

Applied Developmental and Educational Psychology

THE INFLUENCE OF THE FAMILY ENVIRONMENT ON ADAPTIVE FUNCTIONING IN THE CLASSROOM: A LONGITUDINAL STUDY OF CHILDREN WITH DEVELOPMENTAL DISABILITIES

Dissertation

by

MIRIAM HEYMAN

submitted in partial fulfillment

of the requirements for the degree of

Doctor of Philosophy

May 2015 
(C) Copyright by Miriam Heyman 


\title{
The Influence of the Family Environment on Adaptive Functioning in the Classroom: A Longitudinal Study of Children with Developmental Disabilities
}

\author{
Miriam Heyman \\ Dissertation Director: Penny Hauser-Cram, Ed.D.
}

This dissertation utilized data from the Early Intervention Collaborative Study (EICS), a longitudinal study of children with developmental disabilities (DD) and their families (Hauser-Cram, Warfield, Shonkoff, \& Krauss, 2001). The sample for this dissertation consisted of 170 children with DD, their parents, and their teachers. During home visits at ages 2 and 3, mothers and fathers reported on indicators of the home and family environment, and interactions between children and their mothers were observed. At ages 3, 5, 8, 10, and 15, teachers reported on children's levels of classroom-based adaptive functioning. Multilevel modeling was used to examine children's trajectories of classroom-based adaptive functioning. Indicators of the early childhood home and family environment were explored as predictors of these trajectories.

The following research questions were addressed: (1) What are the trajectories of classroom-based adaptive functioning among children with DD from ages 3 to 15? (2) Are levels of adaptive functioning in the classroom stable over time, between the ages of 3 and 15 ? (3) Is there variability in rates of change in adaptive functioning over time, with some children developing more rapidly than others? (4) Do characteristics of the early childhood home and family longitudinally predict children's adaptive functioning in the classroom at age 3 and from ages 3 to15? (5) Which domains of classroom adaptive functioning are predicted by characteristics of the home and family?

Results indicated that children's classroom-based adaptive functioning raw scores increase over time. In each domain of adaptive functioning (socialization, 
communication, and daily living skills) there was significant variability in initial status and rate of change. As hypothesized, quality of early childhood mother-child interaction was predictive of adaptive functioning, with higher quality mother-child interaction associated with more positive functioning. Contrary to hypotheses, the number of negative life events experienced by the family during early childhood was also positively related to classroom adaptive functioning, with more events related to higher levels of functioning. Overall, the findings indicated the influence of the early childhood home and family environment on classroom-based adaptive functioning over time. Policy implications and areas for future research are discussed. 


\section{Acknowledgments}

I would like to express tremendous gratitude to everyone who has made this dissertation possible. I am incredibly grateful to my dissertation chair, Dr. Penny HauserCram, for all of the mentoring that she has provided during my graduate career. The experiences that she has provided, in addition to her support, encouragement, and wisdom, have contributed enormously to my personal and professional development. I would also like to thank my other committee members, Dr. Jacqueline Lerner and Dr. Patrick Proctor. This work would not have been possible without their insight, guidance, and generosity.

I would also like to thank my family, who never stopped believing that I would one day complete my dissertation. To Alex, who provides constant support and perspective. And to Caleb, whose smiles are an invaluable source of encouragement.

Finally, thank you to the Early Intervention Collaborative Study participants. Your time and commitment has made this research possible. 


\section{Table of Contents}

$\underline{\text { Title }}$

$\underline{\text { Page \# }}$

Chapter 1: Problem Statement.................................... 1

Chapter 2: Literature Review.................................. 5

Introduction............................................ 5

Adaptive Functioning...................................... 6

The Early Childhood Home and Family Context.................. 23

Goals of the Current Study.................................. 46

Chapter 3: Methodology.......................................... 49

Participants............................................ 49

Procedure................................................... 53

Measures................................................ 54

Analytic Approach........................................... 67

Chapter 4: Results............................................. 82

Preliminary Analyses................................... 82

Primary Analyses......................................... 92

Chapter 5: Discussion.......................................... 125

Trajectories of Growth.................................. 126

Early Childhood Home and Family Environment Predictors........ 134

Conclusions........................................... 149

References...................................................... 154 


\section{List of Tables}

$\underline{\text { Title }}$

$\underline{\text { Page \# }}$

Table 1: Family and Child Demographic Characteristics at T3..........

Table 2: Teacher Characteristics at Multiple Time Points................

Table 3: List of Measures.......................................... 180

Table 4: Percentage of Missing Data: Child and Family ................. 182

Table 5: Percentage of Missing Data: Teacher and Classroom .............. 183

Table 6: Descriptive Statistics: Continuous Variables.................... 184

Table 7: Descriptive Statistics: Categorical Variables................... 186

Table 8: Correlations Between Adaptive Functioning Raw Scores At

Each Time Point ...........................................

Table 9: Correlations Between Covariates and Adaptive

Functioning Outcomes ..................................... 188

Table 10: Correlations Between Predictors and Adaptive

Functioning Outcomes......................................... 189

Table 11: Correlations Between Predictors and Covariates................. 190

Table 12: Multilevel Models Predicting Socialization....................... 191

Table 13: Multilevel Models Predicting Communication ...................... 192

Table 14: Multilevel Models Predicting Daily Living Skills.................. 193

Table 15: Multilevel Models Predicting Total Scores of Adaptive Functioning...........................................

Table 16: Negative Life Events Experienced by Families ..................... 195 
Table 17: Predicted Values of Adaptive Functioning Raw Scores for Children with Down Syndrome (based on indicators of the early childhood home and family environment) and Corresponding Age Equivalents...................

Table 18: Predicted Values of Adaptive Functioning Raw Scores for Children with Motor Impairment (based on indicators of the early childhood home and family environment) and Corresponding Age Equivalents...................

Table 19: Predicted Values of Adaptive Functioning Raw Scores for Children with Developmental Delay of Unknown Etiology (based on indicators of the early childhood home and family environment) and Corresponding Age Equivalents .................................. 


\section{List of Figures}

$\underline{\text { Title }}$

$\underline{\text { Page \# }}$

Figure 1: Unconditional Model Predicting Socialization Raw Scores......

Figure 2: Unconditional Model Predicting Communication Raw Scores....

Figure 3: Unconditional Model Predicting Daily Living Raw Scores.........

Figure 4: Unconditional Model Predicting

Total Adaptive Functioning Raw Scores........................... 200

Figure 5: Mother-Child Interaction as a Predictor of Socialization Scores for Children with Down Syndrome and Motor Impairment...........

Figure 6: Mother-Child Interaction as a Predictor of Socialization Scores for Children with Developmental Delay of Unknown Etiology........

Figure 7: Negative Life Events as a Predictor of Socialization Scores

for Children with Down Syndrome and Motor Impairment.............

Figure 8: Negative Life Events as a Predictor of Socialization Scores

for Children with Developmental Delay of Unknown

Etiology

Figure 9: Mother-Child Interaction as a Predictor of Communication Scores

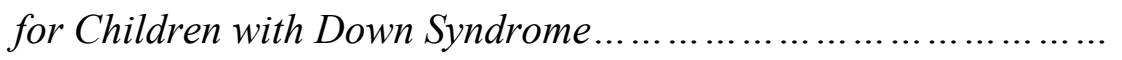

Figure 10: Mother-Child Interaction as a Predictor of Communication Scores

for Children with Motor Impairment ........................................ 203

Figure 11: Mother-Child Interaction as a Predictor of Communication Scores

for Children with Developmental Delay of Unknown Etiology....

Figure 12: Negative Life Events as a Predictor of Communication Scores 
for Children with Down Syndrome

Figure 13: Negative Life Events as a Predictor of Communication Scores

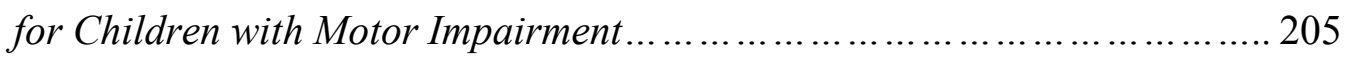

Figure 14: Negative Life Events as a Predictor of Communication Scores

for Children with Developmental Delay of Unknown Etiology................. 205

Figure 15: Mother-Child Interaction as a Predictor of Daily Living Scores

for Children with Down Syndrome.................................. 206

Figure 16: Mother-Child Interaction as a Predictor of Daily Living Scores

for Children with Motor Impairment......................................... 206

Figure 17: Mother-Child Interaction as a Predictor of Daily Living Scores

for Children with Developmental Delay of Unknown Etiology................. 207

Figure 18: Negative Life Events as a Predictor of Daily Living Scores

for Children with Down Syndrome.............................. 207

Figure 19: Negative Life Events as a Predictor of Daily Living Scores

for Children with Motor Impairment........................................ 208

Figure 20: Negative Life Events as a Predictor of Daily Living Scores

for Children with Developmental Delay of Unknown Etiology................. 208

Figure 21: Mother-Child Interaction as a Predictor of Total

Adaptive Functioning Raw Scores for Children with Down Syndrome ........ 209

Figure 22: Mother-Child Interaction as a Predictor of Total

Adaptive Functioning Raw Scores for Children with Motor Impairment ...... 209

Figure 23: Mother-Child Interaction as a Predictor of Total

Adaptive Functioning Raw Scores for Children with Developmental 
Delay of Unknown Etiology

Figure 24: Negative Life Events as a Predictor of Total

Adaptive Functioning Raw Scores for Children with Down Syndrome........ 210

Figure 25: Negative Life Events as a Predictor of Total

Adaptive Functioning Raw Scores for Children with Motor Impairment.....

Figure 26: Negative Life Events as a Predictor of Total

Adaptive Functioning Raw Scores for Children with Developmental

Delay of Unknown Etiology.

211 


\section{Chapter 1: Problem Statement}

Current theoretical approaches to the study of child development stress the interaction between the individual and his or her context (Bronfenbrenner \& Morris, 2006; Lerner, Theokas, \& Jelicic, 2005). Development emanates from these interactions, and therefore in order to understand development, it is crucial to understand factors contributing to the interactions that occur in each of the primary developmental contexts. For many children, these contexts include home and school or other early childhood setting, such as preschool. These are the places that contain the majority of the personenvironment interactions that subsequently shape development.

The level of a person's functioning within a context influences the extent to which that person is able to engage in the type of stimulating interactions that foster development (Bronfenbrenner \& Morris, 2006). For individuals with developmental disabilities (DD), adaptive functioning is especially important to consider. Adaptive functioning includes communication, socialization, and daily living abilities, and it therefore underlies the ability to participate in many significant life activities. While there is a body of research that has investigated adaptive functioning within the home (Dieterich, Herbert, Landry, Swank, \& Smith, 2004; Fenning \& Baker, 2012; HauserCram, Warfield, Shonkoff, Krauss, Upshur, \& Sayer, 1999), research on adaptive functioning within the school is relatively scarce. This is a critical gap in the literature, since positive functioning in the classroom environment is likely a necessary prerequisite for meaningful participation in school-based activities. In turn, meaningful participation in school activities can help prepare individuals for future tasks, such as independent living and employment. 
Because an individual's functioning in a given context contributes to his or her ability to engage in developmentally fruitful interactions, individual characteristics cultivated in one context will influence interactions and related outcomes that transpire in a second (Bronfenbrenner \& Morris, 2006). Research with typically developing children explicitly addresses this component of contextual theories. More specifically, there are relationships between interactions that transpire at home during early childhood and school-based outcomes, including academic achievement and social-emotional functioning in the classroom (Aram \& Aviram, 2009; Baker \& Iruka, 2013). From a theoretical perspective, the home is important due to the importance of early relationships with caregivers. For example, attachment theory posits that young children with sensitive and responsive mothers acquire confidence to explore the world and engage socially with others (Bowlby, 1969). This exploration and social engagement contributes to the achievement of developmental outcomes throughout the life span, thus indicating the primacy of the early childhood home and family environment (Bowlby, 1969). Extant research suggests that dominant theories of development generally apply to children with DD (Hauser-Cram et al., 1999). For children with DD, very few studies explicitly address the hypothesis derived from contextual theories that processes observed in one context result in developmental outcomes manifested in another.

However, there is empirical support for the relationship between the early childhood family environment, including quality of interaction between parents and children, and children's adaptive functioning (Dieterich, et al., 2004; Fenning \& Baker, 2012; Hauser-Cram et al., 1999). Parents might directly teach skills related to adaptive functioning, such as how to bathe, prepare food, give simple directions, etc. Parents 
might also model positive communication behaviors, and provide opportunities to practice these behaviors during routines such as meals. Socialization theory suggests that the quality of parent-child interactions during episodes of teaching, modeling, and practicing likely influences the effectiveness of these episodes (Grusec, Goodnow, \& Kuczynski, 2000). For example, young children are especially motivated to please and imitate parents when the relationship is characterized by warmth, responsiveness, and security (Grusec et al., 2000).

Each study that has linked characteristics of the home and family during early childhood to adaptive functioning measured adaptive functioning via parent-report (Dieterich, et al., 2004; Fenning \& Baker, 2012; Hauser-Cram et al., 1999). As such, these measures of adaptive functioning likely reflect functioning in the context most familiar to the parent: the home. In contrast, there is a dearth of research that has explored adaptive functioning in school, where there are context-specific levels of support, demands, and resources. For example, schools can offer professionals who are specifically trained to provide individually tailored supports for children with disabilities. However, professionals work with several children, and relationships between professionals and children are often limited in duration. Parents have the unique opportunity to gain profound knowledge and understanding of their children over time. They can apply this knowledge to implement supports to enhance the functioning of their children.

This dissertation explores trajectories of classroom adaptive functioning over time. It is important to understand these trajectories because adaptive functioning in the classroom impacts children's abilities to participate meaningfully in classroom activities 
and benefit from opportunities for cognitive, academic, and social/emotional development. This development will be important for achievement of long-term outcomes, including employment, adult relationships, and independent living. This dissertation also investigates relationships between experiences at home and with family members during early childhood and adaptive functioning in the classroom, where task demands undoubtedly differ from the demands within the home and family context. The identification of significant early childhood family environment predictors of schoolbased adaptive functioning has implications for policies related to early intervention services. 


\section{Chapter 2: Literature Review}

\section{Introduction}

Current theoretical approaches to the study of child development emphasize the role of ongoing, bidirectional interaction between an individual and his or her environment (Bronfenbrenner \& Morris, 2006; Lerner et al., 2005). This interaction is shaped by both person and context characteristics, and constitutes a primary force of development (Bronfenbrenner \& Morris, 2006). An individuals' level of functioning in the environment contributes to the interactions that transpire, and is therefore a contributor to subsequent developmental progress. For individuals with DD, adaptive functioning is an indicator of level of functioning within an environment (Widaman \& McGrew, 1996). Because levels of adaptive functioning contribute to individuals' abilities to meaningfully participate in educational and social activities, development in this domain is an essential process. Importantly, adaptive functioning reflects characteristics of the environment in which it is exhibited, including levels of demands and support (Harrison \& Boney, 2002). Therefore, an individual's level of functioning might differ across contexts.

While adaptive functioning is partially context dependent, contextual theories suggest that an individual's level of functioning in one context, such as the school, are partly susceptible to experiences in other environments, including the early childhood home and family (Bronfenbrenner \& Morris, 2006). Attachment theory (Bowlby, 1969) and socialization theory (Bugenthal \& Grusec, 2006) highlight the influence of interactions with caregivers during early childhood. During this developmental phase, 
children develop views of self and others that color their subsequent interactions and opportunities for learning and development.

This review begins with an overview of adaptive functioning, including a description of its conceptualization, importance, and measurement. The review describes research that has investigated trajectories of adaptive functioning for children with DD, and research that has identified predictors of positive development in this domain. Information about adaptive functioning in the classroom context is highlighted as a key gap in the current literature.

The review continues with a discussion about empirical and theoretical support for the relevance of the early childhood home and family context for development in general and adaptive functioning in particular. Indicators of the early childhood home and family context are discussed, and research linking these indicators to school-based outcomes is reviewed. The literature review concludes with goals and hypotheses for this dissertation.

\section{Adaptive Functioning}

\section{Conceptualization, Importance, and Measurement}

Adaptive behavior is defined as "the ability to respond to environmental demands", or "the behavioral skills that people typically exhibit when dealing with the

environmental demands they confront" (Arias, Verdugo, Navas, \& Gomez, 2013, p. 156; Widaman \& McGrew, 1996, p. 97). The terms adaptive behavior and adaptive functioning can be used interchangeably; the level of a person's adaptive functioning refers to the level of typically exhibited skills and behaviors. A crucial component of this definition is its focus on the skills that people typically exhibit; adaptive functioning 
refers to an individual's functioning within the contexts of regular routines and environmental demands.

The definition of adaptive functioning (e.g., "the ability to respond to environmental demands") conveys that a person's level of adaptive functioning will have a pervasive impact on his or her daily experiences, opportunities, and achievements (Arias et al., 2013, p. 156). Adaptive functioning is related to outcomes including employment, friendship, and participation in volunteer activities (Burbidge, Minnes, Buell, \& Ouellette-Kuntz, 2008; Maenner, Smith, Hong, Makuch, Greenberg, \& Mailick, 2013; Matheson, Olsen, \& Weisner, 2007). Furthermore, limitations in adaptive functioning are a necessary condition for diagnosis of intellectual disability (ID) (American Psychiatric Association, 2013). Tasse and colleagues assert that the construct "fulfills four essential functions in the field" of ID (2012, p. 291). These functions include (1) providing information necessary for diagnosis of ID; (2) providing information necessary for differential diagnosis within the category of ID; (3) providing assistance with tracking individual skill development and planning education and related services accordingly; and (4) providing "an essential dimension in a multidimensional understanding of human functioning" (Tasse et al., 2012, p. 291). It is an essential dimension because human beings do not exist outside of environments. Adaptive functioning refers to their ability to confront, interact with, and thrive within these environments.

In light of this importance of the construct, its accurate measurement is critical. There are approximately 200 scales of adaptive behavior, but only four that meet the following criteria: reflect a multi-dimensional factor model, including multiple distinct 
domains of adaptive functioning; have demonstrated adequate reliability and validity; and "have been standardized on people with and without ID" (Arias et al., 2013, p. 157).

These four measures include: (1) the Vineland Adaptive Beahvior Scales $-2^{\text {nd }}$ edition (Sparrow et al., 2005); (2) the Adaptive Behavior Assessment System $-2^{\text {nd }}$ edition (Harrison \& Oakland, 2003); (3) the Scales of Independent Behavior - Revised (Bruininks, Woodcock, Weatherman, \& Hill, 1996); and (4) the Adaptive Behavior Scale - School Version (Lambert, Nihira, \& Leland, 1993). Scores on any of these measures that are two standard deviations below the population mean indicate "significant limitations"; these scores might pertain to a single factor (i.e., daily living, socialization, or communication) or a total score of adaptive functioning (Arias et al., 2013, p. 161).

Current conceptualizations of adaptive behavior presume that it is a multidimensional construct, and this dissertation follows this premise (Tasse et al., 2012). Each dimension within the construct of adaptive functioning can be considered a factor. Factors commonly reflect conceptual skills including "language, reading and writing, and money, time, and number concepts"; practical skills include "activities of daily living (personal care), occupational skills, use of money, safety, health care, travel / transportation, schedules / routines, and use of the telephone"; and social skills encompass “interpersonal skills, social responsibility, self-esteem, gullibility, naivete [i.e., wariness], follows rules / obeys laws, avoids being victimized, and social problem solving" (Tasse et al., 2012, p. 293). Recent research examined children with and without intellectual disability, and demonstrated that a multi-factor model is superior to a unidimensional construct of adaptive functioning (Arias et al., 2013). A multi- factor model, with each domain separate and without a hierarchy, was also superior to a model 
that consisted of three first-order factors loading onto a higher-level second-order factor of overall adaptive behavior (Arias et al., 2013). This finding highlights the relevance of each factor individually, and suggests that it is not necessary to emphasize or quantify the relative contribution of each factor to one singular whole. Accordingly, an individual is considered to have a "significant limitation" in adaptive functioning if he or she exhibits performance that is at least two standard deviations below the population mean on any single factor (Arias et al., 2013, p. 161).

Labels for the factors vary across measurement instruments (Tasse et al., 2012). For example, the Adaptive Behavior Assessment System (Second Edition) measures conceptual, practical, and social factors (Harrison \& Oakland, 2003). Alternatively, the Vineland Adaptive Behavior Scales (Second Edition) measures the communication, socialization, daily living, and motor skills domains. Tasse et al. (2012) endorse both of these instruments for the purpose of diagnosing intellectual disability, and emphasize the importance of an instrument that reflects the "multidimensional conceptual and measurement model of adaptive behavior" (p. 295).

While some measures include motor skills as a factor or domain of adaptive functioning (e.g., the Vineland Adaptive Behavior Scales, Sparrow, Cicchetti, \& Balla, 2005) many current conceptualizations reflect the premise that motor skills should be considered separately, due to relationships between the motor domain and both physical health and developmental status (Arias et al., 2013). Also, while problem behaviors and adaptive behaviors are highly correlated in some groups (such as people with autism spectrum disorders), levels of problem behaviors do not factor into assessments of adaptive behaviors (Arias et al., 2013). This is due to the "general agreement among 
scientists and clinicians that the presence of significant levels of behavior problems does not mean significant limitations in adaptive functioning" (Arias et al., 2013, p. 156). Evidence for the distinction between adaptive functioning and behavior problems includes the fact that while these constructs are highly correlated for some groups (i.e., people with ASD), correlations between these constructs and individuals with ID other than ASD are less salient (Arias et al., 2013). The distinctions between adaptive functioning and behavior problems and adaptive functioning and motor skills are important and appropriate given the fact that significant adaptive functioning challenges are a criterion for diagnosis of ID (Arias et al., 2013). People who exhibit high levels of behavior problems, or low levels of motor skills, are not necessarily assigned a diagnosis of ID. This facilitates proper distinction between categories including developmental delay in the motor domain, ID, and mental health issues.

Adaptive functioning is also moderately correlated with, but distinct from, intelligence (Harrison \& Boney, 2002). While intelligence tests are commonly used in order to assess "academic competence", measures of adaptive functioning are intended to capture "everyday skills" (Harrison \& Boney, 2002, p. 1174). Conceptually, it is possible for an individual to struggle in the academic realm and succeed with tasks that they encounter in their daily routines. Literacy, for example, is a necessary skill for academic success. Literacy can be helpful for adaptive functioning - i.e. the ability to read a recipe in order to prepare food - but it is possible for people to successfully cook for themselves without following a written recipe.

This distinction between intelligence and adaptive functioning relates to an important limitation of intelligence testing. While intelligence tests are purported to 
measure "ability to learn", Tyler argues that their purpose is in fact much more narrow (1984, p. 49). She asserts that "intelligence test scores predict primarily how successful individuals will be with school learning, particularly the more difficult and complex parts of the curriculum" (Tyler, 1984, p. 49). For children with DD, enhancing their ability to function independently in home and work environments is a primary goal, sometimes more important than obtaining high levels of academic achievement. Importantly, Tyler also makes the point that intelligence tests do not measure individuals" "ability to adapt to life's changing demands" (Tyler, 1984, p. 49). For individuals with DD who strive to live and work independently, this is a crucial outcome. An individuals' level of adaptive functioning, defined as "the ability to respond to environmental demands" reflects the extent to which an individual has adapted to an environment, including the demands within (Arias et al., 2013, p. 156).

Finally, adaptive functioning includes the "age-appropriate behaviors necessary for people to live independently and to function safely and appropriately in daily life" (emphasis added; Baghdadli et al., 2012, p. 1314). Therefore, the behaviors that constitute high levels of adaptive functioning change as individuals age and confront different demands in their daily lives. Furthermore, the rank-order stability of adaptive functioning is moderate to strong, meaning that an individual with high levels of adaptive functioning relative to same age peers is likely to maintain this high level over time (Bornstein, Hahn, \& Suwalsky, 2013; Sparrow, Balla, \& Cicchetti, 1984.). Therefore, while exhibited adaptive behaviors are expected to increase in complexity, an individual child is expected to maintain a relatively stable position in relation to his or her same-age peers. 


\section{Trajectories of Adaptive Functioning for Children with DD}

Because children with DD exhibit difficulties related to adaptive functioning, it is important to understand their trajectories in this domain. The interest in trajectories reflects the fact that "the timing, intensity, and type of interventions must be informed by a longitudinal understanding of the problem" (Anderson et al., 2009, p. 1020). Generally, children with DD display growth in adaptive functioning; this has been demonstrated in children with Down syndrome, ASD, motor impairment, and developmental delay of unknown etiology (Baghdadli et al., 2012; Hauser-Cram, Warfield, Shonkoff, \& Krauss, 2001). However, the rate of change is slower than that of typically developing children. Therefore, when comparing children with DD to typically developing children, the gap between levels of typically displayed behaviors increases over time.

Regarding the time frame of growth in adaptive functioning for children with DD, one study of children with Down syndrome has suggested that rates of adaptive functioning reach ceiling levels in early adolescence (van Duijn, Dijkxhoorn, Scholte, \& van Berckelaer-Onnes, 2010). A study of children with ASD, in contrast, indicated that growth does not level off at this same time period (Anderson et al., 2009). In interpreting these findings, it is important to consider that "an important assumption about adaptive functioning is that with appropriate supports and interventions for a person's adaptive skills deficits, life functioning will generally improve" (Harrison \& Boney, 2002, p. 1168). Due to the central role of the environment in supporting positive adaptive functioning, it is likely that with adequate supports, most individuals with and without developmental disabilities can maintain growth throughout development. In support of this possibility, another study of children with Down syndrome indicated that there was 
variability in rate of change between the ages of 6 and 11 (greater variability than existed at a younger age), indicating that growth leveled off for some, but not all, children (Dykens et al., 2006). This variability indicates that a diagnosis of Down syndrome does not absolutely determine whether or not one's adaptive functioning will reach a ceiling level in adolescence. Rather, there are individual differences, which may reflect environmental differences such as quality or quantity of supports to enhance adaptive functioning. For example, elements of the early childhood home environment have predicted growth in adaptive functioning for children with Down syndrome (HauserCram et al., 2001).

While there is potential for most individuals to exhibit growth in adaptive functioning, different disability groups display different typical patterns of growth. In fact, some research has suggested that group differences in adaptive functioning trajectories can be used as indicators in the diagnostic process (Mitchell, Cardy, \& Zwaigenbaum, 2011). For example, young children with ASD, compared to children with other DD, display slower growth in communication and socialization. Children with Down syndrome (when compared to children with other DD) display pronounced growth in socialization trajectories, while children with motor impairment display less pronounced growth in daily living skills (Hauser-Cram et al., 2001). Also, a study that compared trajectories of daily living skills between people with autism and people with Down syndrome, found that both groups increased their skills between adolescence and young adulthood (Smith, Maenner, \& Seltzer, 2012). However, growth for people with ASD slowed at age 20, while growth for people with Down syndrome did not exhibit a similar curve. Due to these differences between disability groups, and due to the 
importance of understanding trajectories in order to design intervention and support, studies have attempted to describe disability-specific trajectories. The study of trajectories, in contrast to the examination of adaptive functioning at single time points, adds valuable information to the developmental knowledge base and also facilitates the implementation of timely intervention. Baghdadli et al. (2012) assessed children with ASD at ages 5, 8, and 15. These authors identified two trajectories for both socialization and communication; one was linear but flat and characterized by low growth, while the other was quadratic with pronounced growth between the ages of 5 and 8 . For daily living, the sample fit one trajectory; a quadratic with relatively sharp growth between ages 5 and 8 (Baghdadli et al., 2012). Implications of these findings include the need to explore why some children displayed pronounced growth in communication and socialization, while others did not. Baghdadli et al. (2012) found that children who displayed pronounced growth in communication and socialization received more hours of intervention between the ages of 5 and 8 than children who displayed low growth. This suggests the important role of timely intervention, in this case between the ages of 5 and 8. For daily living skills, while there were not multiple distinct group trajectories, it is important to consider how to expand the period of pronounced growth, so that it extends beyond 8 years of age.

In addition to trajectory variations across disability categories, research has identified predictors of positive growth in adaptive functioning within disability categories. Cognitive performance has emerged as a predictor of growth rates for children with autism (Anderson et al., 2009; Ben-Itzchak, Watson, \& Zachor, 2014) and cerebral palsy (Vos et al., 2013). Others have suggested that cognitive performance is 
predictive of adaptive functioning because many tasks necessary for adaptive functioning require "a sequence of actions that must be planned beforehand" and rely on "the intellectual ability to anticipate a (changing) environment" (Vos et al., 2013, p. e920). However, as previously described, adaptive functioning and cognitive performance (or IQ) are distinct constructs, with adaptive functioning being particularly dependent on the context (Harrison \& Boney, 2002). While cognitive performance likely facilitates high levels of adaptive functioning, it is possible to be highly intelligent and yet exhibit low levels of adaptive functioning due to the lack of proper environmental supports.

In addition to personal characteristics including cognitive performance, variables related to the environment have also emerged as predictors of adaptive functioning growth. For children with ASD, number of therapy or service hours is related to growth in communication (Baghdadli et al., 2012) and social skills (Anderson et al., 2009). While Baghdadli et al. (2012) found that family functioning did not predict growth in any domain in adaptive functioning for children with ASD ages 5-15, different patterns have emerged for children with Down syndrome. For example, in a study that examined children with Down syndrome from infancy through age five, growth in several domains of adaptive functioning were predicted by elements of the family environment including family cohesion and quality of mother-child interaction (Hauser-Cram et al., 1999). In contrast to results typically found with children with ASD, levels of intellectual functioning did not predict growth in adaptive functioning (Hauser-Cram et al., 1999).

Variables related to socioeconomic status predict positive growth in social skills for children with Down syndrome (Hauser-Cram et al., 1999) and ASD (Anderson et al, 2009). There are several distinct mechanisms through which socioeconomic may 
influence growth in adaptive functioning. Children whose families have extensive socioeconomic resources have an enhanced probability of accessing services, including early intervention services, that directly target skills related to adaptive functioning (Peterson et al., 2004). These families are also relatively likely to provide resources and modifications to enhance the adaptive functioning of their children, including assistive communication devices, ramps, accessible kitchen and bathroom utilities, etc. Also, research with families including children with disabilities has indicated relationships between socioeconomic status and measures of parental well-being, including stress levels (Woodman, 2014). Parental well-being potentially impacts parent-child relationships, which in turn impact child development, including development in adaptive functioning. Interestingly, Baghdadli et al. (2012) did not find significant socioeconomic predictors of growth in their sample of children with ASD. However, these authors did find relationships between hours of early intervention services and growth in adaptive functioning (Baghdadli et al., 2012). Therefore, they found evidence for the relevance of one potential mechanism (described above) that links socioeconomic status and growth in adaptive functioning.

\section{Predictors of Adaptive Functioning}

As conveyed by the literature describing trajectories of adaptive functioning, due to the importance of functioning in this domain, several studies have sought to identify its predictors (e.g., de Bildt, Sytema, Kraijer, Sparrow, \& Minderaa, 2005; Dieterich et al., 2004; Fenning \& Baker, 2012; Hauser-Cram et al., 1999). This line of work is important, because the identification of predictors has valuable implications for intervention design. This section will review the literature on child characteristics that are predictive of 
adaptive functioning, and then address predictive environmental and / or experiential variables, including the home and family context.

For children with developmental disabilities, cross-sectional studies indicate that predictors of adaptive functioning include child characteristics, such as behavior problems (de Bildt et al., 2005), IQ, and inhibitory control (Gligorovic \& Durovic, 2014). Children with lower levels of behavior problems, and higher levels of IQ and inhibitory control display higher levels of adaptive functioning (de Bildt et al., 2005; Gligorovic \& Durovic, 2014). While these variables are predictive of adaptive functioning, as noted above, they each represent constructs that are distinct from adaptive functioning. Therefore, there are mechanisms that link adaptive functioning with each of these predictors. Mechanisms linking behavior problems and adaptive functioning might reflect the fact that both of these constructs are context-dependent; environments that fail to provide the necessary supports for high levels of adaptive functioning might also cause frustration and / or despair which could be manifested in either externalizing or internalizing behavior problems. Regarding IQ, many adaptive functioning skills rely on cognitive processes that are related to IQ, such as memory. Individuals need to remember daily hygiene routines (daily living domain), social norms (socialization domain), and words with which to communicate (communication domain). Finally, inhibitory control likely promotes adaptive functioning abilities because sufficient levels of inhibitory control facilitate the learning and completion of novel and challenging tasks, including tasks related to adaptive functioning. Inhibitory control contributes' to individuals ability to focus on the task at hand despite frustration or temptation to engage with a potential distraction. 
Type of disability is another child characteristic that is predictive of outcomes related to adaptive functioning. For example, many children with Down syndrome have pronounced challenges with communication skills (especially expressive language), relative to social skills and daily living skills (Dykens, Hodapp, \& Evans, 2006). In contrast, children with autism spectrum disorders (ASD) have especially pronounced deficits in social adaptive functioning skills (Anderson, Oti, Lord, \& Welch, 2009). Also for children with ASD, challenges related to adaptive functioning are often larger than would be predicted by individuals' IQ scores (Anderson et al., 2009).

This interaction between IQ and type of disability demonstrates that it is necessary to simultaneously consider multiple child characteristics. Variables including IQ and behavior problems are predictive of adaptive functioning (Arias et al., 2013), yet research indicates that predictive variables differ in their relationships with adaptive functioning across different groups of children. For example, behavior problems and adaptive functioning are relatively highly correlated for individuals with ASD, and relatively less correlated for individuals with ID not including ASD (Arias et al., 2013). One study compared predictors of growth in adaptive social abilities (one domain of adaptive functioning) for children with ASD, pervasive developmental disorder (PDD), and children with other developmental disabilities (Anderson et al., 2009). This study found that early childhood expressive communication was saliently related to socialization growth for children with ASD, while receptive communication was more important for children with PDD. In addition, maternal education was more highly related to adaptive functioning for children with PDD than children with ASD (Anderson et al., 2009). 
Predictors of adaptive functioning also include environmental and experiential factors. For example, research indicates that interventions can produce improvements in adaptive functioning for children with ASD (Flanagan, Perry, \& Freeman, 2012). These researchers utilized a waitlist design to measure the effectiveness of a behavioral intervention based on principles of applied behavior analysis (ABA) for children with autism (Flanagan et al., 2012). At the conclusion of the intervention, participants demonstrated higher gains on a measure of adaptive functioning than individuals on the waitlist. This intervention involved the explicit teaching of skills, many of which relate to adaptive functioning, and "children were consistently taught to generalize their skills to new contexts" (Flanagan et al., 2012, p. 676). Therefore, this intervention likely directly targeted some aspects of adaptive functioning, since children learned to exercise their skills in their natural environments, including the home and school.

Also, for children with a range of developmental disabilities, younger age at entry into early intervention services is related to larger improvements in adaptive functioning (Warfield, 1994). This suggests that early receipt of services is an experiential factor that influences development in this domain. For children with Down syndrome, other relevant environmental predictors include characteristics related to the early childhood home and family, including variables indicative of socioeconomic status, family cohesion, and mother-child interaction (Hauser-Cram et al., 1999). Stimulation from mothers, measured as the "frequency of maternal attention-directing events" during an observation of mother-child interaction, has been linked to more positive trajectories of daily living skills for children born prematurely (Dieterich et al., 2004, p. 288). These authors equated higher levels of stimulation with greater maternal involvement, and 
likely presumed that this involvement was beneficial for children as they sought to acquire new skills. Optimally, parental involvement can serve to quell frustration and encourage perseverance, and it can also be instructional in nature.

Additionally, higher levels of maternal scaffolding have longitudinally predicted socialization skills for children with early developmental delays who were at risk for ID (Fenning \& Baker, 2012). In this study, maternal scaffolding was assessed through observation of mother-child interaction during a teacher task that occurred in a laboratory. The authors speculate that children who benefit from high-quality maternal instruction during specific teaching episodes acquire skills that they are able to transfer to other settings (Fenning \& Baker, 2012). Therefore, high quality maternal teaching enables skill acquisition that transfers to multiple natural environments. For this reason, it likely enhances adaptive functioning.

These findings likely reflect the importance of mothers that provide cognitive stimulation, high quality teaching, and emotional support that encourages participation in challenging activities despite frustration. However, each of these studies that examined the role of environmental and experiential factors measured adaptive functioning via parent-report. As such, these studies utilize measurements of adaptive functioning as displayed when parents are present. The identification of predictors of adaptive functioning at school remains a key gap in the literature. It is essential to identify predictors of adaptive functioning at school in order to design appropriate services, supports, and interventions. This could enhance children's adaptive functioning at school, and facilitate their meaningful involvement in school-based activities.

\section{Adaptive Functioning: The Importance of Context}


The home and school have context- specific levels of support, demands, and resources. These factors are important to consider, because as others have noted, levels of adaptive functioning can be improved with the provision of appropriate supports (Harrison \& Boney, 2002). More generally, the relevance of supports indicates the influence of the environment on adaptive functioning. For example, a parent might be especially adept at providing scaffolding to a child, and the child might exhibit higher skill levels when he or she is able to access help from this parent, or able to access resources that the parent provided, such as checklists, diagrams, and other visual aids. This indicates the interrelationship between context and adaptive functioning. While a body of work has investigated adaptive functioning within the home (Dieterich et al., 2004; Fenning \& Baker, 2012; Hauser-Cram et al., 1999), research on adaptive functioning within the school remains a key gap in the literature. This is important to address, because levels of adaptive functioning at school contribute to individuals' abilities to meaningfully participate in opportunities for academic and social growth.

Parent versus teacher report. The vast majority of research that has investigated trajectories of adaptive functioning has relied on parent report (Anderson et al., 2009; Baghdadli et al., 2012; Dykens et al., 2006; Hauser-Cram et al., 1999; HauserCram et al., 2001; Vos et al., 2013). Also, almost all studies that have identified environmental predictors of adaptive functioning (Anderson et al., 2009; Baghdadli et al., 2012; Dieterich et al., 2004; Fenning \& Baker, 2012; Hauser-Cram et al., 1999; HauserCram et al., 2001; Warfield, 1994) have utilized a parent-report measure. These studies therefore provide valuable information about adaptive functioning typically observed by 
the parent - much of which occurs in the home. However, very little is known about predictors of positive adaptive functioning in the school context.

Dynamics within classrooms are dramatically different than dynamics within families (Parsons, 1959) and this suggests the need to examine children's functioning in both contexts. In a seminal piece addressing the unique dynamics and functions of the classroom, sociologist Talcott Parsons posited that parents rely on age and gender as they form expectations and appraisals of their children (1959). Teachers, on the other hand, compare each child in a class to as many as 25 other children of similar age (Parsons, 1959). Due to these different bases for comparison, it is quite possible for teachers and parents to evaluate children differently (Parsons, 1959). Parsons suggested that these dynamics specific to the classroom suggest the need to examine socialization processes that are unique to the classroom (1959). For example, he explored how individuals' characteristics, including socioeconomic status and ability levels relative to their peers, influence their school-based socialization, or the process through which "individual personalities are trained to be motivationally and technically adequate to the performance of adult roles" (Parsons, 1959, p. 208). As described later in this literature review, socialization processes are posited to contribute to individuals' levels of adaptive functioning. Therefore, differential dynamics across the home and school likely relate to processes, including socialization processes that influence levels of adaptive functioning. Some studies have implicitly addressed contextual considerations by comparing teacher reports of adaptive functioning to parent reports (e.g., Hundert, Morrison, Mahoney, Mundy, \& Vernon, 1997; Voelker, Johnston, Agar, Gragg, \& Menna, 2008; Voelker, et al, 1997; Voelker, Shore, Lee, \& Szuszkiewicz, 2000). In a study of children 
with multiple disabilities ages 3-12, Voelker et al. (1997) found that teachers rated children more highly than parents on each domain of adaptive functioning. There are multiple possible explanations for this discrepancy, including explanations related to differences between parents and teachers (Voelker et al., 1997). For example, teachers are specifically trained to notice demonstrations of skills. A second possibility suggested by the authors is that children's behaviors differ across contexts (Voelker et al., 1997). Parents and teachers each reported about the behaviors that they most commonly observe (in the home and school, respectively) (Voelker et al., 1997). The children in the Voelker et al. study attended school in a rehabilitation day treatment setting, and therefore the school context was highly specialized (1997). As the authors suggest, it is possible that school supports elicited especially high levels of adaptive functioning (Voelker et al., 1997). Finally, given the fact that students' classmates also had multiple disabilities, teachers' points of comparison might have differed from parents' (Parsons, 1959).

This line of work has highlighted important questions about adaptive functioning, context, and the interrelationships between context of adaptive functioning and reporter (parent versus teacher). Specifically, parent and teacher reports of adaptive functioning differ, and these differences likely reflect differences between contexts in which adaptive behaviors are observed. However, describing trajectories of adaptive functioning in school, and identifying predictors of positive functioning in this context, remain key gaps in the literature.

The Early Childhood Home and Family Context General Importance of Early Childhood Home and Family 
According to a bioecological model, development emanates from proximal processes, which consist of interactions between children and the environment (Bronfenbrenner \& Morris, 2006). The proximal processes that have the most influence are the ones that occur frequently over a long period of time (Bronfenbrenner \& Morris, 2006). For many young children, their most frequent and regular interactions are with family members inside of the home. More specifically, due to the importance of nurturing relationships with caregivers during early childhood, and because parents are primary caregivers, others have suggested that "parenting instantiates proximal processes for children" (Bornstein, in press). However, proximal processes are also shaped by other objects in, and characteristics of, the environment (Bronfenbrenner \& Morris, 2006). The availability of toys, space, and organization influence the extent to which children are able to engage in productive and stimulating proximal processes (Bradley \& Caldwell, 1984).

Attachment theory also emphasizes processes that occur in early childhood and the impact of these processes on subsequent development (Bowlby, 1969). For example, from the perspective of attachment theory, infants with available and responsive mothers gain a sense of security (Bowlby, 1969). This security facilitates their ability to engage in exploration and positive social interactions (Bowlby, 1969). While exploring and interacting are important processes for several domains of development, they are particularly relevant for three domains of adaptive functioning: communication, daily living skills, and socialization. Exploring the world provides unique opportunities to practice daily living skills, and novel experiences about which to communicate. Positive social interactions are necessary opportunities to practice and develop socialization skills. 
Bowlby (1973) has also contended that early relationships with caregivers provide infants and young children with working models of themselves and their caregivers. These models are sustained over time and across contexts and influence individuals' relationships and interactions. For children with DD, relationships and interactions in their school environments likely directly influence levels of adaptive functioning.

\section{Empirical Support for the Salience of Early Childhood}

Research with typically developing children indicates that the effects of the early childhood home and family context exert a long-lasting influence. An early study on this topic demonstrated that the early childhood home environment predicted teacher ratings of behavior problems in middle childhood, even after accounting for levels of intermediate child functioning (Sroufe, Egeland, \& Kreutzer, 1990). More recent research has found maternal sensitivity to be an especially salient aspect of the early childhood home environment (Fraley, Roisman, \& Haltigan, 2013; Roisman \& Fraley, 2013). These authors found that maternal sensitivity during early childhood predicts social and academic competence in adolescence (Fraley et al., 2013; Roisman \& Fraley, 2013). This research suggests, moreover, that maternal sensitivity during early childhood has enduring effects; observed relationships to later outcomes were not entirely mediated by transactional processes or by a stable level of maternal sensitivity that continually influenced concurrent outcomes (Fraley et al., 2013; Roisman \& Fraley, 2013).

Specifically, direct relationships between early childhood maternal sensitivity and adolescent social competence remained significant after accounting for social competence at an earlier time point and maternal sensitivity in adolescence (Fraley et al., 2013; Roisman \& Fraley, 2013). 
Studies also indicate the salience of the early childhood family context for children with DD. For example, one study found that family climate and mother-child interaction measured at age 3 predicted growth in adaptive functioning (as measured through parent-report) at age 10 (Hauser-Cram et al., 2001). The authors interpret these findings as an indication that the early family environment sets children on a course of development in adaptive functioning, beginning in the early childhood years but continuing through middle childhood. These authors also suggest that for outcomes related to adaptive functioning, the influence of the early childhood home and family environment might be especially salient and / or visible after the sensorimotor period, when children are able to exhibit more complex skills and functioning levels (HauserCram et al., 2001).

Despite this empirical evidence for the enduring effects of the early childhood home and family environment, little is known about the "dynamic mechanisms that sustain" the influence of early experiences (Roisman \& Fraley, 2013, p. 149). One possibility relates to the enduring salience of young children's internal models of self and other that are heavily influenced by their interactions with their early caregivers (Sroufe et al., 1990). Sroufe and colleagues (1990) suggest that children carry these working models forward in time and across boundaries of home and school. Children who view themselves positively and who perceive adults as available and supportive might be especially able to benefit from developmentally fruitful environments (Sroufe et al., 1990). These children might also be able to exhibit relatively high levels of resilience in stressful circumstances (Sroufe et al., 1990). Also, while research with typically developing children indicates that the enduring effects of the early childhood home and 
family context are manifested in the school setting (Fraley et al., 2013; Sroufe et al., 1990; Roisman \& Fraley 2013), very little is known about whether this transfer occurs for children with DD.

It is important to continue to explore the enduring effects of the early childhood home and family environment, because they have significant policy implications, especially for children with DD. Currently, children with DD from birth to age three are eligible to receive early intervention (EI) services, a system of services that is federally mandated and available in every state (United States Department of Education, 2014). Services are primarily geared towards facilitating children's positive development, and can include training and / or counseling for parents. Therefore, when enduring positive effects of the early childhood environment are found for children with DD, they suggest the potential importance of enhancing EI services that target the predictive characteristics.

\section{Explanations for Early Childhood Home and Family Influences on Adaptive}

\section{Functioning and Related Outcomes}

Socialization. A large body of research has investigated socialization as it relates to typically developing children (Bugenthal \& Grusec, 2006; Parke \& Buriel, 2006). Bugenthal and Grusec define socialization as "the preparation of the young to manage the tasks of social life" (2006, p. 366). Socialization includes development of "cognitive, socioemotional, and behavioral competencies", and many of these competencies are closely related to adaptive functioning skills (Bugenthal \& Grusec, 2006, p. 366). For example, competencies that support management of social tasks include understanding social norms and conventions, and regulating emotions and behaviors in order to act 
accordingly. These competencies are also required for high levels of adaptive functioning, defined as adaptive responses to environmental demands, including social demands.

Within the body of work that has examined socialization of typically developing children, there is a large focus on the role of parents in the socialization process (Bornstein, in press; Bugenthal \& Grusec, 2006; Maccoby, 1992). As Bugenthal and Grusec (2006) contend, society assigns parents this responsibility. Most parents are intrinsically motivated to engage in socialization practices because their children are a primary source of happiness and fulfillment (Bornstein, in press). Parents appreciate that successful socialization can enable children to excel in many domains, including the achievement of academic, professional, and relational goals (Bornstein, in press). Parents are also motivated to socialize their children in order to increase the odds that these children eventually become parents themselves, and thereby genetically provide immortality to the parents (Bornstein, in press). Also, the socialization of children eases the burden on parents (Bugenthal \& Grusec, 2006). As children become increasingly able to engage in sophisticated social tasks, including tasks that are ultimately necessary for employment and independence, they are less reliant on their parents.

In light of the importance of parents as sources of socialization, the role of parents during early childhood is especially critical (Bugenthal \& Grusec, 2006). Socialization scholars assert that the "early years are those in which socialization produces its greatest effects" (Bugenthal \& Grusec, 2006, p. 368). The salience of early childhood socialization is partly due to the relative willingness of young children, compared to older 
children or adolescents, to receive and internalize socialization messages. A leading theorist has described early childhood susceptibility to parental socialization as follows:

Early childhood has long been thought to be a period in the life cycle when humans are especially malleable, a time when they are open to influences they will carry with them - even long after they have left their family of origin. Childhood characteristics thought to be especially plastic to parent- provided experiences range from the language children speak and the foods they prefer to the academics they achieve and the social customs they retain to the politics they follow and the religious beliefs they profess (Bornstein, in press).

Parents who successfully socialize children often display warmth, self-regulation, support for autonomy, and positive control (Bugenthal \& Grusec, 2006). High levels of parental responsivity, including the presence of warmth, attachment in the parent-child relationship, and general responsiveness, are beneficial to socialization outcomes (Grusec et al., 2000). Children likely want to please and imitate warm parents, and they therefore display the desired socialization behaviors (Grusec et al., 2000). Secure attachment within relationships facilitates socialization because it enables children to perceive their parents as legitimate and stable authority figures (Grusec et al., 2000). Finally, responsiveness influences socialization processes because parents elicit behaviors similar to the ones that they themselves exhibit; sympathetic, engaged, and compliant parents teach, model, and demonstrate to their children, who eventually embody these same characteristics (Grusec et al., 2000).

In addition to the influence of parental characteristics on socialization outcomes, it is also important to consider socialization practices. These practices include caregiving, routines, play, observation, and the provision of rewards and punishment (Bugenthal \& Grusec, 2006). These practices are contexts in which children socially engage or observe the social engagement of others. These contexts are therefore opportunities to learn social rules and conventions (Bugenthal \& Grusec, 2006). In the context of caregiving, parents model levels of warmth and responsivity. In routines 
ranging from mealtimes to weddings, children learn about social norms and customs. In a play activity such as hide and seek, children learn about social obligation (to find the person hiding). Children can directly participate in each of these practices, or they might learn as observers - of a sibling following the social customs of a family, for example. Parents can directly reward desired social behaviors, and punish undesirables ones. In summary, the literature on socialization as it relates to typically developing children clearly highlights the role of the home and family environment during early childhood.

Communication. Also for typically developing children, there is a vast amount of support for relationships between the early childhood home and family environment and another element of adaptive functioning - communication (e.g., Hoff, 2003; Murray \& Hornbaker, 1997; Wang \& Dix, 2013). This literature indicates that interactions with parents (primarily mothers) comprise a primary context for language development. The primacy of the mother-child relationship likely reflects the fact that for many young children, mothers are frequent and consistent communication partners, more so than peers or other family members.

The literature reviewed thus far highlights the importance of parents' interactions with their children. Empirical work focusing on language development in typically developing children also highlights the influence of other indicators of the early childhood home and family environment. For example, one study found that families' participation in academic and cultural activities, as well as the availability of developmentally stimulating resources in the home, predicted child language abilities after controlling for socioeconomic status (Miser \& Hupp, 2012). These authors examined the influence of both the home and school on language abilities, and suggest 
the relevance of a "cyclical interaction", meaning that children who receive more stimulation at home elicit more stimulation at school, which in turn contributes to heightened stimulation at home (Miser \& Hupp, 2012, p. 146). This indicates the potential for processes that occur in one context (the home) to impact processes (and subsequent development) that occur in another (the school). Also, families' participation in activities and possession of resources is particularly important during early childhood due to the fact that young children are reliant on their families for these opportunities; in contrast to adolescents, it is difficult for young children to attend cultural events or acquire educational toys without the chaperonage or facilitation of their parents (Leventhal, Dupere, \& Brooks-Gunn, 2009). Additional explanations for observed links between family activities, resources in the home, and language outcomes include the possibility that participating in diverse activities both outside and within the home supplies family members, including children, with novel and sophisticated conversation topics. These conversations become important contexts for language development. Other potential mechanisms include the possibility that stimulating activities and resources, including games and books, facilitate positive family relationships; family members enjoy their time jointly engaged in these activities and resources, and consequently they participate in the types of parent-child interactions that facilitate positive socialization and communication outcomes. Relatedly, parenting practices are constrained by the larger environment, which includes factors such as the availability or scarcity of money to attend events outside of the home or purchase educational resources to use within the home (Bradley, 2002). Generally, this suggests the relevance of the 
early childhood home and family environment that is conceptualized more broadly than specific elements of parent-child interactions.

Children with DD. Historically, the literature on children with DD has focused on the impact of children on their parents, reflecting the assumption that children with DD are sources of strain and stress (Hodapp \& Ly, 2005). More recent work considers the effects of the family on the child with DD. For example, empirical work has examined parents' influence on an outcome that encompasses socialization and communication - adaptive functioning - and suggests that parents play an important role in the development of this domain (Dieterich et al., 2004; Fenning \& Baker, 2012; Hauser-Cram et al., 1999). Family cohesion and elements of parent-child interaction have been linked with outcomes related to adaptive functioning including socialization (Fenning \& Baker, 2012; Guralnick, 1999), daily living skills (Dieterich et al., 2004), and communication (Harris, Kasari, \& Sigman, 1996; McDuffie \& Yoder, 2010; Warren, Brady, Sterling, Fleming, \& Marquis, 2010).

This body of work indicates that similar early childhood home and family characteristics influence both the child with DD and those in typically developing populations (Hauser-Cram et al., 1999). There is also indication that important differences exist across these populations. For example, the typically developing literature conceptualizes parental directiveness as an indicator of a lack support for autonomy. However, for children with DD, parental directiveness might be an adaptive response to the children's needs for additional support (Guralnick, 1999). For children with DD, there might also be unique relationships between joint attention, another element of parent-child interaction, and developmental outcomes, including language 
development. Joint attention occurs when both the parent and the child are attending to the same object or event. Joint attention might be particularly important for language development for children with $\mathrm{DD}$, relative to typically developing children. In the absence of joint attention, many typically developing children can shift their attention to focus on the object that someone else is attending to, and subsequently engage in interactions that focus on this new object. In contrast, children with DD might be unable to flexibly shift and might instead withdraw from interacting with the other person (McDuffie \& Yoder, 2010). While it is important to identify the processes that operate for children with DD specifically, the point remains that the early childhood home and family context is crucial for the development of adaptive functioning within this population.

\section{Indicators of Early Childhood Home and Family Context}

Child evocative effects. Child temperament is "perhaps the most well-researched determinant of parenting behavior" and is defined as "individual differences in emotional, motor, and attentional reactivity to stimulation, and in patterns of behavioral and attentional self-regulation" (Parke \& Buriel, 2006, p. 458; Putnam, Sanson, \& Rothbart, 2002, p. 255). These individual differences are relatively stable over time (Putnam et al., 2002). A large body of research with typically developing children indicates that individual differences associated with temperament exert an influence on family members (Lerner, 1993; Putnam et al., 2002), and this influence extends to patterns of interaction between children and their parents (Parke \& Buriel, 2006). For example, children with difficult temperaments frequently evoke coercion and distress from their parents (Parke \& Buriel, 2006). Research also indicates that similar processes operate for 
children with disabilities (Bostrom, Broberg, \& Bodin, 2011; Gray, Edwards, O’Callaghan, Cuskelly, \& Gibbons, 2013). For example, one study identified unique temperamental profiles among children with intellectual disability, and child temperamental profile was related to the impact of the child on the mother (Bostrom et al., 2011). Specifically, children in the "disruptive cluster" were more likely to have mothers who reported higher levels of parenting stress. While this study did not address possible links between parenting stress and socialization practices, other studies of children with disabilities have documented links between parenting stress and parentchild relationships (Mitchell \& Hauser-Cram, 2010). Taken together, this research indicates the salient impact of child characteristics on the home and family context, for families with a child with a disability as well as for other families.

Home environment. The home environment encompasses more than a physical structure in which a person lives (Bradley, 2002). It includes interactions and relationships between people in the home, as well as physical properties of the dwelling, including physical resources inside of the home. For example, toys within the home "are a major contributor to child learning and acculturation" (Bradley, 2002, p. 301). Therefore, the availability of stimulating toys is a crucial element of the home environment (Bradley \& Caldwell, 1984). However, the ability of the child to productively engage with toys is dependent on factors such as available assistance and space (Bradley \& Caldwell, 1984). For example, puzzle play in the home was found to facilitate positive development of spatial skills (Levine, Ratliff, Huttenlocher, \& Cannon, 2012). However, for these benefits to emerge, it is likely that children need the puzzle itself, as well as a clear space to work on the puzzle, such as a table. They also might 
need assistance, such as adults providing support and encouragement to work through difficult parts. The authors of the study highlight the language input that children received from their parents as they worked on puzzles (Levine et al., 2012). This example shows the relevance of the toy, the physical environment, and the relationship with a supportive adult. Each of these factors is a crucial element of the home environment.

Conceptualizations of the home environment also include opportunities facilitated by family members, many of which occur outside of the home (Bradley \& Caldwell, 1984). Parents organize and execute trips to libraries, zoos, extracurricular activities, etc. (Bradley \& Caldwell, 1984). These activities comprise developmental contexts; children develop interests, vocabularies, and skills as they visit new places and try new things (Bradley, 2002). Relatedly, parents constitute a key element of the home environment and respond to children's experiences that occur within and outside of the home (Bradley, 2002). Parenting tasks, conceptualized as the provision of sustenance, stimulation, socioemotional support, structure, and surveillance, encompass a wide range of interactions and opportunities (Bradley \& Corwyn, 2004). The home environment is similarly broad and complex.

Research with typically developing children has indicated relationships between these facets of the early childhood home environment and later developmental outcomes. For example, preschool scores on home environment indicators were found to differentially link to reading achievement, mathematics achievement, and language arts achievement in elementary school (Bradley et al., 1986). The following indicators were predictive of at least one elementary school academic outcome: stimulation through toys, 
games and reading materials; language stimulation; physical environment; pride, affection and warmth; stimulation of academic behavior; and variety of stimulation (Bradley et al., 1986). Similar relationships have been found between levels of the home environment and cognitive performance on standardized assessments (Bradley \& Caldwell, 1982).

The literature on typically developing children also indicates relationships between the early childhood home environment and nonacademic outcomes, including social and emotional competence. For example, for children born premature, provision of stimulating experiences during early childhood mediated relationships between income and age five behavior problems, perhaps because cognitively stimulating activities also facilitate positive interactions between family members, as they jointly engage in pleasant, interesting, leisure (Linver, Brooks-Gunn, \& Kohen, 2002). Taken together, the literature linking the home environment and both academic and social outcomes indicates the relevance of the home environment for a wide variety of developmental outcomes.

Research with children with DD indicates stability within families of aspects of the home environment over time (Rousey, Wild, \& Blacher, 2002) and relationships between the home environment and developmental outcomes. For example, one study identified links between the family climate at age three, including levels of cohesion within the family, and externalizing and internalizing behavior problems at age five (Mitchell \& Hauser-Cram, 2009). These authors draw from a family systems perspective (Cox \& Paley, 2003) and suggest that a positive emotional climate within a family contributes to the ability of all family members to regulate their emotions. For children, enhanced emotional regulation might lead to lower levels of behavior problems. Also for 
children with DD, the home environment has been linked to adaptive functioning as measured by parent-report (Hauser-Cram et al., 1999). These authors found that for children with Down syndrome, family cohesion predicted growth in the daily living, communication, and socialization domains (Hauser-Cram et al., 1999). These authors suggest that their findings support the relevance of contextual and ecological theories of child development (i.e, Bronfenbrenner \& Morris, 2006; Lerner et al., 2005) for children with DD.

Research therefore indicates that the early childhood home environment is an important developmental context for both typically developing children and children with DD. As described by a prominent scholar of the home environment, "A supportive environment is one that provides guidance or direction for adequate functioning in other environments" (Bradley, 2002, p. 288). It is therefore necessary to consider links between the home environment and functioning in other contexts, including the school.

Cumulative negative life events. Negative life events within a family can include divorce, death of a family member, and loss of a job. While each event can exert an independent, detrimental and multifaceted impact on a family system, the cumulative number of events experienced within a family is an indicator of the extent of impact. For families including young children with disabilities, research has identified links between negative life events and parenting quality (Bradley, Rock, Whiteside, Caldwell, \& Brisby, 1991) as well as maternal stress (Warfield, Krauss, Hauser-Cram, Upshur, \& Shonkoff, 1999). Also for children with disabilities, parental stress has been linked to outcomes including social competence (Guralnick, Neville, Connor, \& Hammond, 2003). Stress likely influences the quality of the interactions that transpire between parents and their 
children, and these interactions comprise crucial developmental contexts and opportunities. Due to the relationships between negative life events, stress, and developmental consequences, negative life events can be considered indicators of "toxic stress" (Shonkoff, 2009). Importantly, negative life events also have concrete implications, such as accompanying financial obligations and constraints, which are likely exacerbated with each additional cumulative event. The role of these implications is important to investigate, since these burdens can exacerbate stress, as well as limit opportunities for participation in developmentally fruitful activities, such as extracurricular lessons and trips.

The inter-relationships between these factors are enduring; studies with typically developing children document relationships between stressful experiences in early childhood and developmental outcomes in adolescence (Doan \& Evans, 2011) and adulthood (Evans, Schamberg, \& McEwen, 2009). For example, Evans et al. (2009) conducted a longitudinal investigation and identified relationships between duration of time in poverty during early childhood and working memory in young adulthood. These relationships, moreover, were mediated by allostatic load, defined as a "biological marker of cumulative wear and tear on the body that is caused by the mobilization of multiple physiological systems in response to chronic environmental demands" (Evans et al., 2009, p. 6545). These findings indicate that the experience of chronic stress results in physiological stress responses, and these physiological responses negatively impact working memory, a component of executive function. Physiological stress responses truncate development in regions of the brain including the hippocampus and the prefrontal cortex, and this might account for the enduring effects of stress during early 
childhood (Evans et al., 2009). While studies of children with DD have not explored the impact of physiological stress responses including allostatic load, research does indicate the enduring effects of family stress in early childhood. For example, one study of children with developmental disabilities and their families identified links between parental stress during early childhood and parent-child relationships during adolescence (Mitchell \& Hauser-Cram, 2010).

Mother-child interaction. Children's interactions with each of their primary caregivers likely influence their subsequent development. However, most research focuses on children's interactions with the more "available" parent - often, the mother (Barnard \& Kelly, 1990). Therefore, the vast majority of knowledge about parent-child interaction is based on research examining children's interactions with their mothers. Mother-child interaction involves "a reciprocal process in which each member of the dyad sensitively observes and adjusts personal behavior to the other member" (Barnard \& Kelly, 1990, p. 278). This conceptualization of interaction includes the following elements: the behaviors of the mother, the behaviors of the child, and the reciprocity of the dyad (Barnard \& Kelly, 1990). Both the child and the mother have specific behaviors in their "repertoires"; thus factors such as skill levels and willingness to engage in behaviors influence interactions (Barnard \& Kelly, 1990). Examples include infants' abilities to alert their mothers to their needs, and mothers' ability to correctly interpret cues (Barnard \& Kelly, 1990). The reciprocity element, or the extent to which dyad members regulate themselves and adapt their behaviors based on the needs, desires, and cues, of and from their interaction partners, has been described as the "basis for a mutually satisfying relationship" (Barnard \& Kelly, 1990, p. 283). 
Maternal responsivity is a key element of mother-child interaction and closely related to the concept of reciprocity. While there are multiple approaches to describing maternal responsivity, responsive behaviors demonstrate "warm acceptance of infants' needs and interests with responses that are prompt and contingent on infants' signals" (Landry, Smith, \& Swank, 2006, pp. 627-628). Research indicates that responsivity is comprised of distinct but related components (Landry et al., 2006). Conceptualization of these components varies across studies, but this conceptualization commonly draws on attachment and sociocultural theoretical frameworks (Landry et al., 2006). From an attachment perspective (Ainsworth, Blehar, Waters, \& Wall, 1978), elements of responsivity including contingency and warmth provide infants with security in order to conduct exploration and subsequent learning (Landry et al., 2006). These responsive behaviors also relate to social and emotional outcomes because warm and contingent caregivers produce facilitate children's willingness to participate in and abide by socialization practices (Grusec \& Goodnow, 1994). From a sociocultural perspective (Vygotsky, 1978), responsive behaviors including following the infant's attentional lead instead of attempting to redirect, and providing appropriate and rich language input, comprise scaffolding that facilitate cognitive growth (Landry et al., 2006). Generally, responsivity enables infants to learn how to cope with stress and novelty, which facilitates the development of self-regulation (Landry et al., 2006). Self-regulation, and trust in the caregiver that results from responsivity, promote exploration, communication, and cooperation (Landry et al., 2006).

Research has suggested that levels of maternal responsivity are related to children's disability status (Barnard \& Kelly, 1990; Brooks-Gunn \& Lewis, 1984). In an 
early study on this topic, researchers compared responsivity across mothers of children with Down syndrome, cerebral palsy, and developmental delays of other origins (BrooksGunn \& Lewis, 1984). Mothers of children with general developmental delays displayed the highest levels of responsivity compared to the other two groups (Brooks-Gunn \& Lewis, 1984). Mental age of the child was the most salient predictor of responsivity; children with higher mental ages had mothers who were more responsive (Brooks-Gunn \& Lewis, 1984). There are multiple possible explanations for these relationships, including the possibility that children with higher mental ages were more adept at sending interpretable cues to which their mothers could respond (Barnard \& Kelly, 1990).

These factors are important to understand and address, since variables associated with maternal responsiveness are predictive of outcomes for typically developing children (Bornstein \& Tamis-LeMonda, 1989; Landry et al., 2006) and children with DD (Fenning \& Baker, 2012; Young \& Hauser-Cram, 2006). Due to links between responsivity and self-regulation, trust, and exploration, responsivity is associated with, and likely causally related to, cognitive and socioemotional outcomes (Landry et al., 2006). These outcomes include mastery motivation (Young \& Hauser-Cram, 2006), preference for novelty, language, play abilities, performance on standardized assessment of cognitive ability (Bornstein \& Tamis-LeMonda, 1989), cooperation, and positive affect (Landry et al., 2006). Research has also indicated that maternal responsiveness is an especially salient predictor of child outcomes for children who are at risk for disability due to very low birth weight (Landry et al., 2006). This suggests that maternal responsivity can at least partially compensate for other developmental risks. Finally, research has provided evidence that maternal responsivity can be enhanced through 
intervention (Landry et al., 2006). This suggests the importance of understanding the role of this attribute in order to target intervention resources effectively.

Maternal directiveness is an additional element of mother-child interaction that warrants attention (Landry, Smith, Swank, \& Miller-Loncar, 2000). Directiveness includes "structured information" about parents' expectations of the child, and does not necessarily allow room for choice (Landry et al., 2000). For very young children, who lack the communication skills necessary to state their goals in order to "work in a communicative learning context", maternal directiveness is thought to facilitate children's understanding of an interaction and thereby support cognitive development (Landry et al., 2000, p. 358). However, as children's language and cognitive skills advance and directiveness becomes less essential, it is thought to interfere with children's autonomy and subsequently their learning in interactional contexts (Landry et al., 2000). One study found that directiveness was positively associated with children's cognitive and social competence at age two, but the association became negative by age three (Landry et al., 2000). Therefore, benefits of maternal directiveness vary with child age (and presumably, level of development). This likely reflects the relevance of child characteristics such as skill levels.

Due to this relevance of skill levels, it is important to understand the role of directiveness in mother-child dyads in which the child has a disability. One study examined nine year old children (with disabilities and without) and their mothers as they jointly completed a problem solving task (Wieland, Green, Ellingsen, \& Baker, 2014). Results indicated that mothers of children with disabilities displayed more directiveness than mothers of typically developing children (Wieland et al., 2014). The children with 
disabilities in the sample displayed lower expressive skills than the typically developing children, and maternal directiveness was not associated with less warmth or positive affect (Wieland et al., 2014). These findings lend support to the possibility that for children with disabilities, directiveness might be adaptive. However, other studies have found no differences in directiveness when comparing mothers of typically developing children to mothers of children with developmental disabilities (i.e., Gilmore, Cuskelly, Jobling, \& Hayes, 2009). Another study utilized a problem solving task and examined parental directiveness in a sample that consisted of children with Prader-Willi syndrome and Williams syndrome (Ly \& Hodapp, 2005). These authors found that parents of children with Williams syndrome were more directive than parents of children with Prader-Willi syndrome (Ly \& Hodapp, 2005). The authors suggest that children with Williams syndrome required more assistance, and therefore elicited relatively high levels of directiveness (Ly \& Hodapp, 2005). Taken together, this research suggests that levels of directiveness are partially in response to children's needs. High levels of directiveness, when appropriately matched to children's skill levels and need for scaffolding or guidance, are likely adaptive. In these circumstances, directiveness can be conceptualized as appropriate responsivity. Therefore, directiveness and responsivity are not necessarily contradictory or mutually exclusive.

Methods used to assess mother-child interaction, including maternal responsivity and directiveness, include parent report and observation (Barnard \& Kelly, 1990). While parent report is efficient with regard to time and money, it is also subject to biases associated with social desirability (Barnard \& Kelly, 1990). Observation methods include global rating scales, which are intended to reflect the general quality of the 
interaction, and systematic coding schemes, which quantify levels and frequencies of specified behaviors (Barnard \& Kelly, 1990). The extensive resources associated with these observation methods are often warranted given the developmental benefits associated with interventions designed to enhance patterns of mother-child interaction (Landry et al., 2006). These interventions are particularly important in light of indication that without intervention, indicators of mother-child interaction, including maternal responsivity, exhibit stability over time (Bornstein \& Tamis-LeMonda, 1989).

\section{Early Childhood Home and Family Predictors of School-Based Outcomes}

Research with typically developing children indicates that the influence of the family environment extends into the classroom setting. For example, maternal warmth and cognitive stimulation at school-entry are related to reading and math achievement at the end of kindergarten (Baker \& Iruka, 2013), and levels of support and stimulation at home are negatively related to behavior problems at school for elementary school students (O’Connor, Dearing, \& Collins, 2011). Importantly, research with typically developing children also indicates that these effects on school-based outcomes might be enduring, reaching all the way into adolescence (Fraley et al., 2013; Roisman \& Fraley, 2013). One explanation for the enduring nature of these effects is the possibility that children with high quality early caregiving experiences develop positive perceptions of self and positive expectations for others. Children likely carry these perceptions and expectations across time and setting, and their ability to respond adaptively to the school environment is enhanced accordingly (Sroufe et al., 1990). Children who view themselves as competent and who view others as potential sources for care and assistance are able to take advantage of positive environmental opportunities (Sroufe et al., 1990). 
These children are also able to cope with environmental demands and stressors (Sroufe et al., 1990).

In summary, extant research with typically developing children indicates the following about the transfer of influence from the early childhood home and family to the school setting: early childhood home and family indicators are longitudinally predictive of school-based academic and social-emotional outcomes (Baker \& Iruka, 2013; O'Connor et al., 2011); these effects often persist into adolescence and long-term effects are not entirely mediated by transactional processes or continuity of home and family characteristics (Fraley et al., 2013; Roisman \& Fraley, 2013); salient characteristics of the early childhood home and family context include indicators related to parent-child relationships, such as levels of maternal sensitivity (Fraley et al., 2013; Roisman \& Fraley, 2013).

Findings that indicate the influence of the family environment on school-based outcomes are consistent with bioecological models of development (e.g., Bronfenbrenner $\&$ Morris, 2006). Furthermore, research with children with DD indicates that these theoretical models apply to this population (Hauser-Cram et al., 1999). Very few studies including children with DD have explicitly tested this hypothesis that home-based processes exert an influence on school-based outcomes, but there is some evidence for this relationship. For example, for children who exhibited developmental delays in cognitive performance at age 3 , maternal scaffolding at age four is related to teacherrated social skills at age six (Baker, Fenning, Crnic, Baker, \& Blacher, 2007).

However, it is also important to consider the crucial differences between the home and school contexts, since these differences might influence the transfer of skills and 
characteristics. Parents and teachers have different expectations for children; this partially reflects differences between parents and teachers in background knowledge about the child and who the child is being consciously or unconsciously compared to (siblings versus classmates). Importantly, research indicates that teachers' expectations influence the children's actual achievement (i.e., Robinson-Cimpian, Lubienski, Ganley, \& Copur-Gencturk, 2014). Other differences between home and school include levels and types of support, including assistance, encouragement, and physical accessibility. As other have noted, levels of adaptive functioning are often largely dependent on this support (Harrison \& Boney, 2002). More research is needed in order to investigate the relevance of the early childhood home and family environment as a longitudinal predictor of school-based outcomes for children with DD, including adaptive functioning.

\section{Goals of the Current Study}

This dissertation is the first study to investigate trajectories of classroom-based adaptive functioning for children with disabilities. This dissertation is also one of few studies to address links between the early childhood home and family context and schoolbased outcomes for children with disabilities (see Baker et al., 2007 for an example of an extant study that has addressed these links). The dissertation builds upon the extant theory and literature that indicates the following: (1) the influence of the early childhood home and family environment for home-based adaptive functioning for children with DD (e.g., Fenning \& Baker, 2012; Hauser-Cram et al., 2001) and related outcomes including socialization for typically developing children (e.g., Bornstein, in press; Bugenthal \& Grusec, 2006); (2) the salient and enduring effects of the early childhood home and family environment for typically developing children (e.g., Bowlby, 1973; 
Bronfenbreener \& Morris, 2006; Roisman \& Fraley, 2013; Sroufe et al., 1990) and children with DD (Hauser-Cram et al., 2001); (3) and the transfer of influences from the early childhood home and family to the school setting (e.g., Baker et al., 2007; Bronfenbrenner \& Morris, 2006). This dissertation explores the following research questions: (1) What are the trajectories of classroom-based adaptive functioning among children with DD from ages 3 to 15? (2) Are levels of adaptive functioning in the classroom stable over time, between the ages of 3 and 15? (3) Is there variability in rates of change in adaptive functioning over time, with some children developing more rapidly than others? This study also seeks to explain extant variability with early childhood home and family predictors. Research questions that address this purpose include: (4) Do characteristics of the early childhood home and family longitudinally predict children's adaptive functioning in the classroom at age 3 and from ages 3 to15? (5) Which domains of classroom adaptive functioning are predicted by characteristics of the home and family?

The hypotheses corresponding to these research questions are as follows:

Hypothesis 1: There will be increases in raw scores on classroom adaptive functioning on each domain, and on total scores of adaptive functioning, over time.

Hypothesis 2: There will be variability between children with regard to mean levels and rates of change of classroom adaptive functioning (each domain and total scores) over time.

Hypothesis 3: The early childhood family environment will predict this variability, after accounting for classroom characteristics and dynamics, child characteristics, and family socioeconomic status. Predictive home and family characteristics will include variables 
related to child evocative effects, mother-child interaction, negative life events, and a measure of learning opportunities within the home. There will be significant relationships between these variables and each of the three following domains of adaptive functioning: communication, socialization, and daily living skills. There will also be significant relationships between these variables and children's total scores of adaptive functioning. 


\section{Chapter 3: Methodology}

\section{Participants}

This dissertation utilizes data from the Early Intervention Collaborative Study (EICS), a longitudinal investigation of children with developmental disabilities and their families (Hauser-Cram, et al., 2001). EICS was initiated in 1985 in order to explore the adaptation of children with DD and their families over time. Families enrolled in EICS when they originally signed up to receive Early Intervention Services in 29 publicly funded programs in Massachusetts and New Hampshire. This original sample consisted of 190 children and families. All children had early diagnosed DD, including Down syndrome $(\mathrm{N}=54)$, motor impairment $(\mathrm{N}=77)$, or developmental disability of unknown etiology $(\mathrm{N}=59)$. Diagnosis of Down syndrome was confirmed via review of medical records. Children with motor impairment displayed delayed or abnormal motor development and either abnormal muscle tone or a coordination deficit. The children with DD of unknown etiology displayed delays in at least two domains of development. These three disability categories (Down syndrome, motor impairment, DD of unknown etiology) were the most common among recipients of early intervention services in Massachusetts at the time of study enrollment (in 1985). Data were collected at multiple time points during home visits and classroom observations through questionnaires, interviews, and assessments. Home visits occurred when the children with DD were ages $1,2,3,5,10,18$, and 23. Classroom observations and / or teacher interviews occurred when the children were ages $3,5,8,10$, and 15 .

The sample for this dissertation includes 170 children, their families, and their teachers. Each of these 170 children received school-based services pertaining to their 
disabilities at one or more teacher interview time points (ages 3, 5, 8, 10 and 15).

Adaptive functioning in the classroom was measured at ages 3, 5, 8, 10, and 15; each of the children in the sample had an IEP or a 504 plan at one or more of these time points. Each of these children also has data representing at least one measurement of adaptive functioning in the classroom. The majority of this sample $(93.5 \%)$ has data representing classroom-based adaptive functioning at more than one age. Approximately eighty two percent $(82.4 \%)$ of the sample has data representing at least three measurements of adaptive functioning. The extent of missing data will be described in more detail in a subsequent section.

This subsample (170 children, with their respective families and teachers) represents $89.5 \%$ of the original EICS sample. At each individual time point for which there are data available about school-based services or accommodations, the majority of children were eligible for these services or accommodations. Approximately half of the children (53.5\%) had an IEP at age 3, 70\% of children had an IEP at age $8,70 \%$ of children had an IEP at age 10, and $100 \%$ of the children had either an IEP or a 504 plan (or there was a plan in progress) at age 15. More than one third of the children $(37.1 \%)$ had an IEP or 504 plan at all of these time points.

The fact that all of the children had an IEP or 504 plan (or there was a plan in progress) at age 15 does not necessarily indicate a rise in service eligibility. At earlier ages, data only reflect whether or not there was an IEP. At age 15, data reflect one of three possibilities: IEP, 504 plan, or a plan in progress. Therefore, the age 15 data represent broader criteria. Finally, the relatively low percentage of children with IEPs at age 3 likely reflects the fact that these children were still transitioning into the public 
school system from the early intervention service system. This transition is complex, with necessary steps including referral, screening, and eligibility evaluations, since not all children who qualify for early intervention services will quality for special education services (Lillie \& Vakil, 2002; Malone \& Gallagher, 2008). The transition culminates in the creation of an IEP, but due to complexities inherent to the transition process, many families experience delays in their receipt of IEPs and a subsequent delay in receipt of services.

Table 1 displays a summary of child and family characteristics. Approximately one half of the 170 children are of each gender ( $54.7 \%$ male), and approximately one third are in each of the three disability categories (30.6\% Down syndrome, 39.4\% motor impairment, and $30.0 \%$ other DD). The majority of the children are of Euro-American descent (91.5\%). At age 3, the children's mean cognitive performance score was measured via the Stanford Binet Intelligence Scales (Thorndike, Hagen, \& Sattler, 1986) and was equal to $60.34(\mathrm{SD}=23.05)$. This is more than two standard deviations below the population mean for the measure $(\mathrm{M}=100 ; \mathrm{SD}=15)$. Also at age 3 , mothers reported on their children's adaptive functioning through completion of the Vineland Adaptive Behavior Scales-Interview Form (Sparrow et al., 1984). The mean score on this measure was equal to $64.68(\mathrm{SD}=12.67)$. This represents more than two standard deviations below the measure's population mean $(\mathrm{M}=100 ; \mathrm{SD}=15)$.

When the families initially enrolled in the study, on average, mothers and fathers had respectively completed an average of 13.85 and 13.99 years in school (SD $=2.42$ for mothers, 3.02 for fathers). In 1990, the median income in the United States was equal to $\$ 29,943$ (United States Census Bureau, 2012), and 51\% of families in this sample 
reported incomes higher than $\$ 30,000$ when the children were 3 years old (data collected between 1989 and 1991). Approximately one third (31.3\%) reported family incomes greater than $\$ 40,000$. Therefore, most families were middle income. Also when the children were 3 years old, the majority of mothers $(81.7 \%)$ and fathers $(84.6 \%)$ were married, and most children (81.9\%) lived with both of their parents. Mothers had a mean age of 31.59 years $(\mathrm{SD}=5.13)$ and fathers were slightly older $(\mathrm{M}=33.87, \mathrm{SD}=5.86)$. The average number of other children in the family (in addition to the focal child) was equal to $1.22(\mathrm{SD}=1.02)$.

Table 2 displays a summary of teacher characteristics for each time point during which classroom-based adaptive functioning was measured (when the children were ages $3,5,8,10$, and 15). When the children were in preschool (ages 3 and 5), the vast majority of the teachers were female. Most of the teachers at these time points had degrees in special education (75.9\% and 70.3\%). Also, at ages 3 and 5 approximately half of the teachers had Bachelor's degrees (50.7\% at age 3 and 51.4\% at age 5). Most of the remaining teachers had Master's degrees (41.1\% at age 3 and $38.5 \%$ at age 5$)$. Preschool teachers at ages 3 and 5 had approximately seven years of experience (at age 3 , $\mathrm{M}=6.68, \mathrm{SD}=6.08$; at age $5, \mathrm{M}=6.80, \mathrm{SD}=5.22$ ).

At ages 8 and 10, teachers had approximately fifteen and thirteen years of experience $(\mathrm{M}=15.25, \mathrm{SD}=9.66$ at age $8 ; \mathrm{M}=13.73, \mathrm{SD}=8.62$ at age 10$)$. Compared to the preschool teachers, fewer middle childhood teachers had degrees in special education. At age $8,45.7 \%$ of teachers had this specialized degree, and $55.7 \%$ of teachers at age 10 had a degree in special education. Similar to the teachers from the 
earlier time points, most teachers at ages 8 and 10 had a Bachelor's degree $(51.52 \%$ at age 8 and $32.8 \%$ at age 10 ) or a Master's degree (36.2\% at age 8 and $46.6 \%$ at age 10$)$.

Different information was collected from teachers at age 15 , due to the unique roles of teachers at this point in children's educational experiences. The majority of teachers at age 15 were special education teachers $(65.4 \%)$. The remaining teachers were subject teachers $(9.0 \%)$, subject and special education teachers $(9.0 \%)$, aides or assistants (7.7\%), or some other type of school personnel, such as guidance counselors.

\section{Procedure}

Data pertaining to the early childhood home and family environment were collected at home visits by trained research assistants. Home visits occurred within six months of the children's first, second, and third birthdays. Research assistants were trained in order to achieve reliability on all study measures. The assistants were blind to the hypotheses of the study. Home visits included multidimensional structured assessments of the children and of the mother-child dyads. Mothers and fathers also responded to interview questions and completed several self-administered questionnaires. Each home visit was approximately two to three hours in duration. Parents were compensated for their participation in the home visits.

Data pertaining to the school were collected when the children were ages $3,5,8$, 10, and 15. Trained research assistants, who were trained to be reliable on study measures and were blind to the study's hypotheses, completed classroom observations. School-based data also included self-administered questionnaires which were to be completed by the teachers. The University of Massachusetts Medical School Institutional Review Board (IRB) approved the procedure for the data that were collected at ages 3 
and 5. The Brandeis University IRB approved the procedures for data collected at age 8, and the Boston College IRB approved the procedure for ages 10 and 15.

\section{Measures}

\section{Early childhood home and family context.}

Child and family demographic information. At the time of enrollment in the

EICS study, families provided demographic information including the gender of the child with a disability and the type of disability diagnosis (Down syndrome, motor impairment, developmental disability of unknown etiology). Researchers reviewed medical records in order to confirm diagnosis of Down syndrome. Children with motor impairment had delayed or abnormal motor development and either abnormal muscle tone or a deficit in coordination. The children with DD of unknown etiology had delays in at least two domains of development. Gender and type of disability are included in equations predicting classroom-based adaptive functioning as covariates (see Table 3). Gender was coded as follow: $1=$ male, $0=$ female.

Type of disability was recoded such that Down syndrome comprises the reference group. This is due to research findings suggesting that individuals with Down syndrome have unique characteristics related to adaptive functioning (Dykens et al., 2006). In order to create a Down syndrome reference group, two new variables were created: motor impairment and other $D D$. Individuals with Down syndrome received a value of zero in each of these variables. Individuals with motor impairment received a value of 1 for the motor impairment variable, and a value of 0 for the other DD variable. Individuals with other DD received a value of 0 for the motor impairment variable, and a value of 1 for the other DD variable. 
During home visits when the children with DD were 3 years old, mothers provided demographic information about the family, including family income and maternal years of education. Family income and maternal years of education were recoded into z-scores, and a composite variable was formed by summing the two zscores. This composite variable represents the socioeconomic status of the family, and is included as a covariate.

Child evocative effects. Current theoretical approaches to the study of child development emphasize the ongoing, bidirectional influences of the environment on the child and the child on the environment (Lerner et al., 2005; Sameroff, 1995). Therefore, it is imperative to account for the impact of the child on the early childhood home and family context.

During home visits when the children with DD were 3 years old, mothers and fathers each completed the Parenting Stress Index (Abidin, 1995).The Parenting Stress Index (PSI) was designed for the "early identification of stressful parent-child systems" (Abidin, 1995, p. 1). It yields a score of total stress, as well as a score for stress in the child domain and a score for stress in the parent domain. Parent domain subscales measure parental perceptions of their own competence, isolation, attachment, health, role restriction, depression, and relationship with their spouse. Child domain subscales include parental perceptions of the child's distractibility / hyperactivity, adaptability, the extent to which the child reinforces the parent, demandingness, mood, and acceptability. The following three child domain subscales are particularly relevant to child evocative effects, and are utilized in this dissertation: adaptability, demandingness, and mood. For most of the items on each of these subscales (described in more detail below), parents 
indicated the extent to which they agreed with the item. Options ranged from 1 (strongly agree) to 5 (strongly disagree). A few items utilize five-point Likert scales with unique response options (i.e., $1=$ almost always, $5=$ almost never).

The adaptability subscale consists of eleven items, and "high scores in this area are associated with characteristics that make the parenting task more difficult by virtue of the child's inability to adjust to the changes in his or her physical or social environment" (Abidin, 1995, p. 8). Sample items include "My child reacts very strongly when something happens that my child doesn't like" and "It takes a long time and it is very hard for my child to get used to new things".

The demandingness subscale consists of nine items, and "high scores in this area are produced when the parent experiences the child as placing many demands upon him or her" (Abidin, 1995, p. 9). Demands can have multiple manifestations, and the items represent this diversity. Sample items include "My child has had more health problems than I expected" and "My child is always hanging on me".

Finally, the mood subscale is intended to identify children "whose affective functioning shows evidence of dysfunction" (Abidin, 1995, p. 9), meaning that they frequently exhibit distress relative to the frequency with which they display happiness. Five items comprise this subscale, including "When playing, my child doesn't often giggle or laugh" and "My child generally wakes up in a bad mood".

For this dissertation, total scores were calculated for both mothers and fathers. For each parent, the total scores represent the sum of the adaptability, demandingness, and mood subscales, with higher sores indicating more parental difficulty related to these characteristics of their child's evocative effects. For mothers and fathers, Cronbach's 
alpha for the total scores are 0.87 and 0.86 , respectively. Mothers' and fathers' total scores are highly correlated $(r=0.56, p<0.01)$. Each of the two total scores were recoded into z-scores, and a composite variable was formed by summing the two z-scores. This composite score therefore represents the child's evocative effects on multiple family members: the mother and the father. Higher values of the composite indicate more stressful child evocative effects.

Home environment. During home visits when the children with DD were 2 years old, trained research assistants completed the Home Observation for the Measurement of the Environment (H.O.M.E) (Bradley \& Caldwell, 1984). While the measurement of mother-child interaction (described below) occurred one year later, research has suggested the stability of the home environment over time (Rousey et al., 2002).

The H.O.M.E is an observational measure that has demonstrated reliability and validity in assessing "the stimulation potential of the early developmental environment" (Caldwell \& Bradley, 1984). According the measure's manual, the H.O.M.E. was administered to a group of 174 families with infants and toddlers residing in Little Rock, Arkansas (Caldwell \& Bradley, 1984). This sample provided evidence that the measure demonstrates indicators of reliability including internal consistency and stability over time (Caldwell \& Bradley, 1984). Evidence of validity includes significant correlations in hypothesized directions between H.O.M.E. subscales and socioeconomic status and mental test scores of infants and toddlers (Caldwell \& Bradley, 1984).

To complete the H.O.M.E., researchers respond to a series of 45 items to indicate whether or not each item was observed during the home visit $(1=$ yes, $0=$ no $)$. Items refer to physical properties of the home (i.e., "child has a special place for toys and 
treasures") and behaviors that caregivers might elicit (i.e., "parent does not express annoyance with or hostility to child"). Items are organized into the following subscales: (1) emotional and verbal responsivity of parent; (2) acceptance of child's behavior; (3) organization of the environment; (4) provision of play materials; (5) parent involvement with child; and (6) physical punishment. Items on the physical punishment subscale are reverse coded. There is also a composite score, representing the overall quality of the home environment.

In this dissertation, this measure is intended to represent the overall and general quality of the home environment. Therefore, the total score is used in analyses as a predictor of classroom-based adaptive functioning. Cronbach's alpha for the total H.O.M.E. inventory in the current sample is equal to 0.79 .

Negative life events. During home visits when the children with DD were 1, 2, and 3 years old, mothers responded to a series of 19 items from the Life Events Scale (Abidin, 1995). For each item, mothers indicated whether or not a specified event had occurred within the last 12 months within the immediate family $(1=$ yes, $0=$ no $)$. A subset of 10 of these events are considered especially stressful and deleterious (divorce, loss of a job, death of a family member, etc.). These are considered the negative life events. The total number of negative life events that occurred during the early childhood period is equal to the sum of the negative life event scores from each of the three time points. This is an indicator of stress within the family system, and is used as a predictor of classroom-based adaptive functioning.

Mother-child interaction. During home visits when the children with DD were 3 years old, mothers and their children completed the Nursing Child Assessment Teaching 
Scale (NCATS) (Barnard, 1978). To complete the NCATS, mothers taught their children how to play with a toy that was moderately challenging for the child. Toys were selected for individual children in order to provide moderate difficulty levels. Trained research assistants observed the interactions and coded for the presence or absence of 50 behaviors that mothers might exhibit, as well as 23 additional behaviors that children might exhibit ( $1=$ yes, $0=$ no). The 50 maternal behaviors comprise the following four subscales: sensitivity to cues, response to distress, social-emotional growth fostering, and cognitive growth fostering.

The items on the response to distress scale (i.e., "stops the teaching episode") are only applicable if the child exhibits distress during the teaching episode. Sensitivity to cues items include "parent praises child's successes or partial successes", and "parent positions the child so that it is possible for them to have eye-to-eye contact with one another during the teaching episode." Social-emotional growth fostering items include "parent laughs or smiles at child during teaching", and "parent gently pats, caresses, strokes, hugs, or kisses child during episode". Finally, cognitive growth fostering items include "parent uses both verbal and nonverbal instructions in teaching the child" and "parent signals completion of the task to the child verbally or nonverbally." Note that the NCAST was developed for use with either mothers or fathers, and therefore items refer to 'parents'. The data for this dissertation consists exclusively of mother-child dyads.

The 23 child behaviors comprise the following two subscales: clarity of cues (i.e., "child smiles or laughs during the teaching episode") and responsiveness to parent (i.e., "child attempts to engage parent in eye-to-eye contact"). NCAST yields scores on each subscale and total scores for both parent and child; the parent's total score is the sum of 
the four relevant subscales, and the child's total score is the sum of the two relevant subscales. Higher scores indicate more observed behaviors and higher quality of motherchild interaction. Finally, there is a contingency score for both parent and child. Each contingency score represents the number of behaviors that one member exhibits that are contingent on the other person. For example, the following item is a parental behavior that is contingent on child performance: "Parent verbally praises child after child has performed better or more successfully than the last attempt."

In this dissertation, the mother's total score is included as a predictor of classroom-based adaptive functioning $(\alpha=0.82)$. The NCAST has demonstrated good internal reliability (Sumner \& Spietz, 1994). In addition, it has demonstrated test-retest reliability scores of 0.85 for parent total score and 0.55 for the child total score (Sumner \& Spietz, 1994).

\section{Classroom characteristics and dynamics.}

Classroom placement type. At the first four time points that included a measurement of classroom-based adaptive functioning (ages 3, 5, 8, and 10), teachers completed a questionnaire on which they indicated the proportion of children in the class with IEPs. This reflects the extent to which the child spent time alongside typically developing peers, versus attending a segregated educational context. This is an important characteristic of the classroom context to address, because it likely relates to factors including extent of support available to the child, teachers' extent of expertise regarding disability, and the skill levels of classmates to whom the teacher is likely to compare the focal child. Each of these factors might influence teacher-report of adaptive functioning. 
The age 15 classroom characteristic variable is unique because at this age, children do not spend their entire days with the same group of peers. School personnel did complete questionnaires at this time point, but since data were collected from only one school staff person per child, asking that one person about class composition would yield data that reflect the composition of a class that the child with DD spends relatively little time with. At age 15, mothers completed a questionnaire that asked them to select the best description of their child's school day, with options including the following: (1) day treatment or residential program, (2) special education with lunch separate from typically developing peers, (3) special education with lunch alongside typically developing peers, (4) special education plus time in employment and / or the community, (5) one to two hours of general education with the remainder of time in special education, (6) one to two hours of special education with the remainder of time in general education, (7) general education with support, and (8) general education without support.

Each of these variables (percentage of children in the class with IEPs at ages 3, 5, 8,10 and description of children's school day at age 15) were coded to reflect whether or not the focal child was in a substantially separate setting. If more than $70 \%$ of the class had IEPs (at ages 3, 5, 8, and 10), this was considered substantially separate. At age 15, response options 1-5 are considered substantially separate, while response options 6-8 are considered inclusive. Data were coded 1 for substantially separate and 0 for not substantially separate.

Classroom externalizing behaviors. Barbara Keogh (1982) has argued that for children with DD, "behavioral styles" in the classroom are an important component of classroom dynamics. For example, research has indicated that children with DD display 
unique temperamental profiles in the classroom compared to their peers without disabilities (Keogh \& Burstein, 1988). Within a group of children with DD, temperamental differences in the classroom have been found to relate to patterns of interaction between children and their peers as well as their teachers (Keogh \& Burstein, 1988).

In this dissertation, levels of externalizing behaviors are used as a measure of children's evocative effects in the classroom. Children's levels of externalizing behaviors are likely associated with the quality of their relationships with their teachers (Eisenhower, Blacher, \& Bush, 2015); the quality of these relationships may influence teachers' ratings of children's adaptive functioning.

At ages 3 and 5, the measure of classroom externalizing behaviors consists of the Kohn Problem Checklist (Kohn, 1988). This measure is designed for the classroom assessment of children between the ages of 3 and 6 , and its use is intended to identify "clinically significant problem behaviors" (Kohn, 1988, p. 6). The Kohn problem checklist consists of 49 items; for each item teachers indicated whether the item described a behavior that was not at all typical, somewhat typical, or very typical (not at all typical $=0$; somewhat typical $=1$; very typical $=2$ ). Teachers are instructed to base their responses on behaviors that they observed during the most recent week.

The 49 items are divided into two factors: the apathy-withdrawal factor (25 items) and the anger-defiance factor (24 items). In this dissertation, the anger-defiance factor is used as the measure of classroom externalizing behaviors. Items include "disobeys directions or instructions of adults", "is restless and unable to sit still", and "bullies, hits or picks on other children." 
The Kohn Problem Checklist has demonstrated internal consistency, interrater reliability, and external validity. In one study, teachers from two private schools in San Antonio, Texas rated 112 children. Reliability coefficients were high for both factors ( $r=$ 0.88 for the apathy-withdrawal factor and 0.93 for the anger-defiance factor) (Kohn, 1988). Spearman-Brown interrater reliability coefficients were also high; 0.73 for both apathy-withdrawal and anger-defiance (Kohn, 1988). Regarding external validity, one longitudinal study demonstrated that earlier scores on the Kohn Problem Checklist significantly and positively correlate with scores one year later on the Classroom Behavior Inventory, a measure of extroversion-introversion and love-hostility (Kohn, 1988; Schaefer \& Aaronson, 1966). For the current sample, at age 3 Cronbach's alpha on the anger-defiance factor is equal to 0.84 . At age 5 , Cronbach's alpha is equal to 0.91 .

At ages 8, 10, and 15, the Teacher Version of the Child Behavior Checklist comprises the measure of classroom externalizing behaviors (Achenbach \& Edelbrock, 1983). To complete this measure, teachers responded to 112 items describing children's behaviors. For each item, they indicated whether it was not true, somewhat or sometimes true, or very true or often true $(0=$ not true, $1=$ somewhat or sometimes true, $2=$ very true or often true). In responding to each item, teachers were instructed to consider the behaviors they had observed within the past two months. The 112 items are divided into 8 subscales: anxious, social withdrawal, unpopular, self-destructive, obsessivecompulsive, inattentive, nervous-overactive, and aggressive. The 8 subscales comprise 3 scales: internalizing, externalizing, and mixed. The externalizing scale, consisting of the inattentive, nervous-overactive, and aggressive subscales, represents the measure of classroom externalizing behaviors at ages 8,10 , and 15 . Items on the inattentive subscale 
include "poor schoolwork" and "disturbs others"; nervous-overactive items include "hyperactive" and "fidgets"; and aggressive items include "disrupts class", "easily frustrated", and "destroys own things".

The Teacher Version of the Child Behavior Checklist externalizing scale has demonstrated excellent reliability and validity (Edelbrock \& Achenbach, 1984). Testretest reliability was assessed with a sample of boys ages 6-11, all of whom attended a school for "disturbed children" (Edelbrock \& Achenbach, 1984, p. 213). Product moment correlations representing test-retest reliability were equal to 0.93 for one week, 0.77 for 2 months, and 0.54 for 4 months (Edelbrock \& Achenbach, 1984). Regarding external validity, scores on this measure have been shown to correlate with observational ratings of both on-task behavior and problem behavior (Reed \& Edelbrock, 1983). Cronbach's alpha for the current sample (on the externalizing scale) is equal to 0.95 at age $8,>0.99$ at age 10 , and 0.93 at age 15 .

Adaptive functioning. The dependent measure consists of the total score, and scores from the daily living, communication, and socialization domains, of the Vineland Adaptive Behavior Scales - Classroom Edition (VABS - CE, Sparrow, Balla, \& Cicchetti, 1985). This measure also includes a motor skills domain, but recent conceptualizations of adaptive functioning have posited that motor skills constitute a separate construct (Arias et al., 2013). Teachers completed the VABS-CE when the children were ages $3,5,8,10$, and 15 .

The VABS-CE was standardized using a nationally representative sample of 2,084 children (Sparrow et al., 1985). This standardization sample has provided evidence for the reliability and validity of the measure. Cronbach's alphas for the communication 
domain ranged from 0.88 to 0.95 across several age groups; they ranged from 0.92 to 0.96 for the daily living skills domain; and Cronbach's alphas for the socialization domain ranged from 0.91 to 0.96 (Sparrow et al., 1985). Evidence for the validity of the measure included age-related increases in raw scores (Sparrow et al., 1985).

Furthermore, factor analyses indicated support for the overall construct of adaptive behavior as well as for the specified domains (Sparrow et al., 1985). Finally, correlations between scores on the VABS-CE and other measures of adaptive functioning and intelligence tests were significant and in the hypothesized direction (Sparrow et al., 1985).

In addition to scores from the daily living, communication, and socialization domains, the measure yields a total adaptive functioning score, as well as scores from subscales within each of the domains. The communication subscales include receptive (i.e., "points accurately to all body parts when asked"), expressive (i.e., "Uses sentences of four or more words"), and written communication (i.e., "addresses envelopes completely"). The daily living subscales include personal (i.e., "covers mouth and nose with hand, tissue, or handkerchief when coughing or sneezing"), domestic (i.e., "puts possessions away when asked, without having to be told the proper places") and community skills (i.e., "correctly counts change from purchase costing more than a dollar"). Finally, the socialization subscale includes coping skills (i.e., "controls anger or hurt feelings when denied own way"), interpersonal skills (i.e., "shows a relatively consistent preference for some friends over others"), and engagement in play and leisure (i.e., "has a hobby"). As noted above, this dissertation utilizes the total score, and scores from the daily living, communication, and socialization domains. 
Teachers responded to 244 items in the self-administered VABS-CE questionnaire by indicating whether the child was able to complete a task never, sometimes, or usually (never $=0$, sometimes $=1$, usually $=2$ ). Also for each item, teachers could indicate whether they had actually observed the child trying to complete the task, or whether their rating was an estimate based on their knowledge of the child's capabilities. According to the measure's manual, even responses that were estimates are considered reflections of adaptive skills within the school context (Sparrow et al., 1985). These estimates are based on what teachers knew about the child, and much of this knowledge originated from observing the child in the classroom. This questionnaire is designed to take approximately twenty minutes to complete.

Cronbach's alphas for the current sample are as follows. For the daily living skills domain, alphas for time points $3,5,8,10$, and 15 are equal to $0.99,0.98,0.99,0.97$, and 0.99 . For the socialization domain, alphas for time points $3,5,8,10$ and 15 are equal to $0.97,0.97,0.97,0.85$, and 0.98 . For the communication domain, alphas for time points $3,5,8,10$ and 15 are equal to $0.98,0.98,0.98,0.98$, and 0.99 . For the total score of adaptive functioning, alphas for each time point are 0.99 or higher. Raw scores for each domain and for the total score at each time point are the dependent variables in multilevel analyses. The decision to use raw scores aligns with other studies that have investigated adaptive functioning in children with DD (Gabriels, Ivers, Hill, Agnew, \& McNeill, 2007; VanMeter, Fein, Morris, Waterhouse, \& Allen, 1997). The use of raw scores allows the avoidance of problems associated with standard scores in research that includes children with DD. These problems include floor effects, since scores are standardized according to a typically developing population (Hessl et al., 2009). Also, 
research that has examined trajectories of adaptive functioning in individuals with DD has suggested that raw scores, in comparison to standard scores, are more likely to reveal actual growth (Gabriels et al., 2007).

\section{Analytic Plan}

Missing data. Multiple imputation was conducted prior to analyses. Multiple imputation is a method of handling missing data, and it is recommended when the amount of missing data is moderate to large (i.e., more than 5\%; Widaman, 2006). There are several alternatives to multiple imputation, including listwise deletion (Widaman, 2006). Listwise deletion consists of the deletion of all cases that are missing at least one variable included in an analysis. Most computer programs utilize listwise deletion by default (Widaman, 2006). However, there are several drawbacks to listwise deletion, including the fact that sample sizes will differ across analyses (because cases might have complete data for some but not all analyses). This will make it difficult to compare results from multiple analyses (Widaman, 2006). Also, with large amounts of missing data, sample sizes for analyses can be greatly reduced, resulting in reduced power to detect relationships (Widaman, 2006). Finally, the cases with missing data might differ systematically from the cases with complete data. For example, one study examined factors affecting attrition in a longitudinal study on the effects of a smoking intervention (Siddiqui, Flay, \& Hu, 1996). Participants who remained in the study had higher levels of academic achievement, and blacks were more likely than other ethnic groups to be in the attrition group. This potential for systematic differences between cases with and without missing data means that if cases with missing data are deleted, estimates of population parameters will be biased accordingly (Widaman, 2006). 
Multiple imputation addresses these limitations of listwise deletion, as well as limitations of other methods including pairwise substitution, sample mean substitution, individual mean substitution, and regression substitution (Widaman, 2006). Single imputation (recommended when amount of missing data is approximately $1 \%-2 \%$ ) and multiple imputation (recommended for moderate to large amounts of missing data) provide estimates for missing values based on information from data that is non-missing (Widaman, 2006). Imputation estimates are also based on a random component, in order to account for the fact that with complete data sets, relationships between variables are not perfect or certain (Widaman, 2006). Single imputation results in one complete data set, whereas multiple imputation results in multiple (usually between five and 10) complete data sets (Widaman, 2006). In each multiply- imputed data set, the values for the non-missing data remain the same. However, the imputed values for the missing data differ across data sets, because different random components are added each time a value is imputed (Widaman, 2006). Data sets are then pooled and analyses are conducted with complete data.

Tables 4 and 5 contain the percentage of missing data for each of the variables in this dissertation. Table 4 contains the percentage of missing data for each of the early childhood home and family variables, and Table 5 contains the percentage of missing data for each of the teacher and classroom measures, including classroom-based adaptive functioning. The amount of missing data for the early childhood home and family differences is relatively low $(0 \%-4.1 \%)$. However, due to the longitudinal design of this study, attrition occurred between early childhood and the last school-based measurement, which occurred at age 15 . Widaman (2006) considers $10 \%-15 \%$ a moderate amount of 
missing data, and $25 \%$ and higher a large amount of missing data. The teacher and classroom measures are within or close to this range (12.4\% - 28.8\% missing), with the exception of the measure of externalizing behavior at age 15 (55.9\% missing).

According to Widaman, multiple imputation is appropriate for data sets with large amounts of missing data, including variables (such as the age 15 externalizing behavior score) that are missing values for more than fifty percent of cases (2006).

Widaman (2006) recommends the imputation of five to 10 data sets for multiple imputation. For this dissertation, prior to conducting analyses, five data sets were imputed and results were pooled. Imputation was conducted using SPSS version 22. All variables included in analyses (listed in Table 3) were also included during the process of imputation. Child cognitive performance, measured at ages $3,5,10$, and 15 was also included in the imputation. At ages 3 and 5, child cognitive performance was measured with the McCarthy Scales of Children's Abilities (McCarthy, 1972). At ages 10 and 15, the Stanford-Binet IV was used to assess cognitive performance (Thorndike et al., 1986).

Main analyses. Hierarchical linear modeling (HLM) was conducted using SPSS version 22 in order to test the hypotheses of this dissertation. HLM is an appropriate methodology because it does not require independence of cases, which is appropriate when multiple observations are from the same participant (Hox, 2010). In this dissertation, multiple measurements of classroom-based adaptive functioning are nested within individuals. HLM also allows the inclusion of both between-person predictors (predictors that do not vary over time, or level-2 predictors) and within-person predictors (also referred to as level-1 predictors), which may change as a function of time. Covariates and predictors representing the early childhood home and family context do 
not vary over time, and are considered level-2 predictors. Level-2 covariates include child's gender, child's type of disability, and family socioeconomic status. Level-2 predictors include the home learning environment, child evocative effects, negative life events, and mother-child interaction. The covariates representing classroom characteristics and dynamics (specifically, classroom externalizing behaviors and the variable indicating whether the child was in an inclusive or substantially separate setting) vary over time, since children were in different classrooms at different time points. Therefore, these variables are considered level-1 predictors.

In order to facilitate interpretation of significant coefficients, methodologists recommend centering of continuous predictors and covariates for HLM analyses (Bickel, 2007). To center a variable, the value of the sample mean is subtracted from each individual's score on the variable (Bickel, 2007). The following continuous predictors and covariates for this dissertation are centered: the composite variable representing socioeconomic status, the measure of classroom externalizing behavior at each time point, the composite variable representing mothers' and fathers' ratings of children's evocative effects, the score representing the quality of mother-child interaction, the number of negative life events experienced by the family, and the score representing the quality of the home learning environment.

The main analyses proceed in several steps. Each step listed below was repeated four times, one time for each of the four outcomes: classroom-based socialization, communication, daily living skills, and total scores of adaptive functioning. First, an unconditional growth model will allow examination of trajectories of classroom-based adaptive functioning. Multiple measurements of classroom-based adaptive functioning 
(at multiple child ages) permit the estimation of trajectories. The unconditional growth model includes child age (a time varying predictor, because it was measured at multiple time points) as the only predictor of classroom-based adaptive functioning. Age is centered at 36 months, meaning that each measurement of child age is converted to months, and then 36 is subtracted from each measurement. Thirty six months is the age of the first measurement of classroom-based adaptive functioning, and therefore centering the data at 36 produces an intercept with a meaningful interpretation. The intercept becomes the average level of adaptive functioning at the initial adaptive functioning measurement. Age is entered as a linear term in order to test the hypothesis that raw scores increase over time. Age is also entered as a squared term, in order to account for the possibility that growth reaches ceiling levels (van Duijn, Dijkxhoorn, Scholte, \& van Berckelaer-Onnes, 2010). The unconditional growth model is as follows:

$$
Y_{t i}=\beta_{00}+\beta_{10}\left(A G E_{t i}\right)+\beta_{20}\left(A G E S Q_{t i}\right)+r_{0 i}+r_{1 i}\left(A G E_{t i}\right)+e_{t i}
$$

where $\mathrm{Y}$ is the outcome (adaptive functioning), $\beta_{00}$ is the intercept (initial status at age 3 ), $\beta_{10}$ is the rate of change in the linear age term, and $\beta_{20}$ is the rate of change in the squared age term. This unconditional model is repeated four times for the four different outcomes: communication, socialization, daily living skills, and total adaptive functioning scores. Results from the unconditional linear growth model are used to test hypotheses 1 and 2 as follows:

Hypothesis 1: Raw scores for each domain of adaptive functioning, and the raw score for the total score of adaptive functioning, will increase over time.

Analysis. A positive and statistically significant coefficient for the linear age term $\left(\beta_{10}\right)$ will indicate support for this hypothesis. A statistically significant coefficient for 
the squared age term $\left(\beta_{20}\right)$ will neither support nor negate this hypothesis, but will suggest that longitudinal growth in adaptive functioning is not steady, but rather exhibits distinct periods of change and stability.

Hypothesis 2: There will be variability between people with regard to mean levels and rates of change of classroom adaptive functioning over time.

Analysis: The unconditional growth model provides estimates for variability in initial status (adaptive functioning at age $3, \hat{\sigma}_{0}^{2}$ ) and variability in growth in adaptive functioning, $\hat{\sigma}_{1}^{2}$. If the value of $\hat{\sigma}_{0}^{2}$ is statistically significant, this will lend support for the hypothesis that the variability in initial status is significantly significant and different than zero. If the value of $\hat{\sigma}_{1}^{2}$ is statistically significant, this will support the hypothesis that variability in rate of change is statistically significant and different than zero.

Hypothesis 3: The early childhood family environment will predict this variability, after accounting for classroom characteristics and dynamics, child characteristics, and family socioeconomic status. Predictive home and family characteristics will include variables related to child evocative effects, mother-child interaction, negative life events, and a measure of learning opportunities within the home. There will be significant relationships between these variables and each of the three domains of adaptive functioning (communication, socialization, and daily living skills) and the total score of adaptive functioning.

Analysis. If the linear and squared terms are both significant, they will both be retained in models testing relationships between the early childhood home and family environment and classroom-based adaptive functioning. However, if either of these terms is not significant, that term will be dropped from subsequent models. 
Covariates will be added to the models in order to account for relations between these covariates and initial status and rates of change in adaptive functioning. These covariates include child gender, child type of disability, classroom placement type (substantially separate versus inclusive setting), classroom levels of externalizing behavior, and the composite representing the family's socioeconomic status. The equations that follow assume that the unconditional growth models indicated significant variability in initial status and rates of change. If there is not significant variability in either estimate for a particular domain of adaptive functioning, or for the total score of adaptive functioning, the addition of covariates will be adjusted accordingly. For example, for daily living skills, if there is variability in initial status but no variability in the rate of change, covariates will be added as predictors of initial status only. The following equation represents the analysis that will be conducted four times, once for each domain of adaptive functioning, and once for the total score of adaptive functioning:

$$
\begin{aligned}
& Y_{t i}=\beta_{00}+\beta_{01}\left(G E N_{i}\right)+\beta_{02}\left(D D_{i}\right)+\beta_{03}\left(M I_{i}\right)+\beta_{04}\left(S E S_{i}-\overline{S E S} .\right) \\
& +\beta_{10}\left(C L A S S_{t i}\right)+\beta_{20}\left(E X T_{t i}\right)+\beta_{30}\left(A G E_{t i}\right)+\beta_{40}\left(A G E S Q_{t i}\right)+\beta_{31}\left(A G E_{t i} * G E N_{i}\right)+ \\
& \beta_{32}\left(A G E_{t i} * D D_{i}\right)+\beta_{33}\left(A G E_{t i} * M I_{i}\right) \\
& +\beta_{34}\left[A G E_{t i} *\left(S E S_{i}-\overline{S E S} \cdot\right)\right]+\beta_{35}\left(A G E_{t i} * C L A S S_{t i}\right)+\beta_{36}\left(A G E_{t i} * E X T_{t i}\right) \\
& +r_{0 i}+r_{1 i}\left(A G E_{t i}\right)+e_{t i}
\end{aligned}
$$

where $\mathrm{Y}$ is the outcome (adaptive functioning), $\beta_{00}$ is the intercept (initial status at age 3 ), $\beta_{30}$ is the rate of change in the linear age term, and $\beta_{40}$ is the rate of change in the squared age term. Predictors of the intercept include the following: $\beta_{01}$ is the coefficient representing gender (with females comprising the reference group), $\beta_{02}$ is the coefficient representing the 'other DD' group (with individuals with Down syndrome comprising the reference group), $\beta_{03}$ is the coefficient representing motor impairment group (with 
individuals with Down syndrome comprising the reference group), $\beta_{04}$ is the coefficient representing socioeconomic status, $\beta_{10}$ is the coefficient representing classroom type (substantially separate versus inclusive setting), and $\beta_{20}$ is the coefficient representing classroom externalizing behaviors. If $\beta_{01}$ is significant and positive, this will indicate that males have a higher initial status than females, with the difference in initial status corresponding to the value of the $\beta_{01}$ coefficient; if $\beta_{02}$ is significant and positive, this will indicate that children with 'other DD' have a higher initial status than children with Down syndrome, with the difference equal to the value of the $\beta_{02}$ coefficient; if $\beta_{03}$ is significant and positive, this will indicate that children with motor impairment have a higher initial status than children with Down syndrome, with the difference equal to the value of the $\beta_{03}$ coefficient. A positive and statistically significant coefficient associated with the socioeconomic status composite $\left(\beta_{04}\right)$ will indicate that for each unit above the mean value of the socioeconomic status composite, individuals' initial adaptive functioning scores are predicted to increase by the value of the coefficient. A negative and statistically significant coefficient associated with classroom type $\left(\beta_{10}\right)$ will indicate that children in substantially separate settings will have lower initial rates of adaptive functioning, with the predicted difference equal to the value of the coefficient. A negative and statistically significant coefficient associated with classroom externalizing behavior problems $\left(\beta_{20}\right)$ will indicate that for each unit higher that a child scores on the measure of these problems, his or her initial status is predicted to decrease by the value of the coefficient. 
Predictors of the slope include the following: $\beta_{31}$ represents the influence of gender on rate of change, (with females comprising the reference group), $\beta_{32}$ is the coefficient representing the influence of membership in the 'other DD' group on rate of change (with individuals with Down syndrome comprising the reference group), $\beta_{33}$ is the coefficient representing the influence of membership in the motor impairment group on rate of change (with individuals with Down syndrome comprising the reference group), and $\beta_{34}$ is the coefficient representing the influence of socioeconomic status on rate of change. The coefficient $\beta_{35}$ will indicate whether the relationship between classroom placement type and adaptive functioning changes over time, and the coefficient $\beta_{36}$ will indicate whether the relationship between classroom externalizing behavior problems and adaptive functioning changes over time. If $\beta_{31}$ is positive and significant, this will indicate that males have a more rapid rate of growth than females, with the difference in rate of change equal to the value of the coefficient. If $\beta_{32}$ is positive and significant, this will indicate that people in the 'other DD' group have a more rapid rate of growth than children with Down syndrome, with the difference in rate of change equal to the value of the coefficient. If $\beta_{33}$ is positive and significant, this will indicate that children with motor impairment have more pronounced growth than children with Down syndrome, with the difference in rate of change equal to the value of the coefficient. If $\beta_{34}$ is positive and significant, this will indicate that for each unit above the mean value of the socioeconomic status composite, individuals' rates of change of adaptive functioning are expected to increase by the value of the coefficient. A significant coefficient associated with $\beta_{35}$ will indicate an interaction between time and classroom type; if the coefficient is 
positive, this will indicate that the influence of classroom type increases over time. A positive and significant coefficient associated with $\beta_{36}$ will indicate that the influence of externalizing behaviors increases over time. The analysis will be repeated four times for the four different outcomes: communication, socialization, daily living skills, and total score of adaptive functioning.

Finally, indicators of the early childhood home and family environment will be added as predictors of both initial status and rate of change of adaptive functioning. Nonsignificant covariates (from the previous model) will be dropped, and significant covariates will be retained. Assuming that all covariates from the previous model were significant, the final models will be as follows:

$$
\begin{aligned}
& Y_{t i}=\beta_{00}+\beta_{01}\left(G E N_{i}\right)+\beta_{02}\left(D D_{i}\right)+\beta_{03}\left(M I_{i}\right)+\beta_{04}\left(S E S_{i}-\overline{S E S} \cdot\right) \\
& +\beta_{05}\left(E V_{i}-\overline{E V} \bullet\right)+\beta_{10}\left(C L A S S_{t i}\right)+\beta_{20}\left(E X T_{t i}\right)+\beta_{06}\left(N L E_{i}-\overline{N L E}_{\bullet}\right)+\beta_{07}\left(H O M_{i}-\overline{H O M} \bullet\right)+ \\
& +\beta_{08}\left(M C I_{i}-\overline{M C I} \cdot\right)+\beta_{30}\left(A G E_{t i}\right)+\beta_{40}\left(A G E S Q_{t i}\right)+\beta_{31}\left(A G E_{t i} * G E N_{i}\right)+\beta_{32}\left(A G E_{t i} * D D_{i}\right) \\
& +\beta_{33}\left(A G E_{t i} * M I_{i}\right)+\beta_{34}\left[A G E_{t i} *\left(S E S_{i}-\overline{S E S} \cdot\right)\right]+\beta_{35}\left[A G E_{t i} *\left(E V_{i}-\overline{E V} \cdot\right)\right]+\beta_{36}\left(A G E_{t i} * C L A S S_{t i}\right) \\
& +\beta_{37}\left(A G E_{t i} * E X T_{t i}\right) \\
& +\beta_{38}\left[A G E_{t i} *\left(H O M_{i}-\overline{H O M}_{\bullet}\right)\right]+\beta_{39}\left[A G E_{t i} *\left(M C I_{i}-\overline{M C I}_{\bullet}\right)\right]+\beta_{310}\left[A G E_{t i} *\left(N L E_{i}-\overline{N L E}_{\bullet}\right)\right] \\
& +r_{0 i}+r_{1 i}\left(A G E_{t i}\right)+e_{t i}
\end{aligned}
$$

where $\mathrm{Y}$ is the outcome (adaptive functioning), $\beta_{00}$ is the intercept (initial status at age 3 ), $\beta_{30}$ is the rate of change in the linear age term, and $\beta_{40}$ is the rate of change in the squared age term. Predictors of the intercept include the following: $\beta_{01}$ is the coefficient representing gender (with females comprising the reference group), $\beta_{02}$ is the coefficient representing the 'other DD' group (with individuals with Down syndrome comprising the reference group), $\beta_{03}$ is the coefficient representing motor impairment group (with individuals with Down syndrome comprising the reference group), $\beta_{04}$ is the coefficient 
representing socioeconomic status, $\beta_{05}$ is the coefficient representing child evocative effects, $\beta_{10}$ is the coefficient representing classroom placement type, $\beta_{20}$ is the coefficient representing classroom externalizing behaviors, $\beta_{06}$ is the coefficient representing negative life events, $\beta_{07}$ is the coefficient representing the quality of the home learning environment, and $\beta_{08}$ is the coefficient representing mother-child interaction.

If $\beta_{01}$ is significant and positive, this will indicate that males have a higher initial status than females, with the difference in initial status corresponding to the value of the $\beta_{01}$ coefficient; if $\beta_{02}$ is significant and positive, this will indicate that children with 'other DD' have a higher initial status than children with Down syndrome, with the difference equal to the value of the $\beta_{02}$ coefficient; if $\beta_{03}$ is significant and positive, this will indicate that children with motor impairment have a higher initial status than children with Down syndrome, with the difference equal to the value of the $\beta_{03}$ coefficient. A positive and statistically significant coefficient associated with the socioeconomic status composite $\left(\beta_{04}\right)$ will indicate that for each unit above the mean value of the socioeconomic status composite, individuals' initial adaptive functioning scores are predicted to increase by the value of the coefficient. A negative and statistically significant coefficient associated with child evocative effects $\left(\beta_{05}\right)$ will indicate that for each unit of higher stress associated with child evocative effects above the sample mean, initial status of change of child adaptive functioning is predicted to decrease by the value of the coefficient. A negative and statistically significant coefficient associated with classroom type $\left(\beta_{10}\right)$ will indicate that children in 
substantially separate settings will have lower initial rates of adaptive functioning, with the predicted difference equal to the value of the coefficient. A negative and significant coefficient associated with classroom externalizing behaviors $\left(\beta_{20}\right)$ will indicate that for each unit higher that a child scores on the measure of these behaviors, his or her adaptive functioning initial status is expected to decrease by the value of the coefficient. If $\beta_{06}$ is negative and significant, this will indicate that for each negative life event than an individual experienced above the mean number of negative life events in the sample, that individual's initial score of adaptive functioning is predicted to decrease by the value of the coefficient. A positive and statistically significant coefficient representing the home environment $\left(\beta_{07}\right)$ will indicate that for each unit representing the quality of the home environment above the mean score of the sample, a child's initial adaptive functioning score is expected to increase by the value of the coefficient. A positive and statistically significant coefficient representing mother-child interaction $\left(\beta_{08}\right)$ will indicate that for each unit above the sample mean on the NCAST score, an individual's initial status of adaptive functioning is expected to increase by the value of the coefficient.

Predictors of the slope include the following: $\beta_{31}$ represents the influence of gender on rate of change, (with females comprising the reference group), $\beta_{32}$ is the coefficient representing the influence of membership in the 'other DD' group on rate of change (with individuals with Down syndrome comprising the reference group), $\beta_{33}$ is the coefficient representing the influence of membership in the motor impairment group on rate of change (with individuals with Down syndrome comprising the reference group), $\beta_{34}$ is the coefficient representing the influence of socioeconomic status on rate 
of change, $\beta_{35}$ is the coefficient representing the influence of child evocative effects on rate of change, $\beta_{36}$ is the coefficient representing the interaction between time and classroom placement type, and $\beta_{37}$ is the coefficient representing the interaction between time and classroom externalizing behaviors. The coefficient $\beta_{38}$ represents the influence of the home environment on rate of change, $\beta_{39}$ is the coefficient representing the influence of mother-child interaction on rate of change, and $\beta_{310}$ is the coefficient representing the influence of negative life events on rate of change.

A positive and significant value associated with $\beta_{31}$ will indicate that males have a more rapid rate of growth than females, with the difference in rate of change equal to the value of the coefficient. If $\beta_{32}$ is positive and significant, this will indicate that people in the 'other DD' group have a more rapid rate of growth than children with Down syndrome, with the difference in rate of change equal to the value of the coefficient. If $\beta_{33}$ is positive and significant, this will indicate that children with motor impairment have more pronounced growth than children with Down syndrome, with the difference in rate of change equal to the value of the coefficient. If $\beta_{34}$ is positive and significant, this will indicate that for each unit above the mean value of the socioeconomic status composite, individuals' rates of change of adaptive functioning are expected to increase by the value of the coefficient. If $\beta_{35}$ is negative and significant, this will indicate that for each unit of higher stress associated with child evocative effects above the sample mean, rate of change of child adaptive functioning is predicted to decrease by the value of the coefficient. A positive and significant coefficient associated with $\beta_{36}$ will indicate that the influence of classroom type increases over time, and a positive and significant coefficient associated 
with $\beta_{37}$ will suggest that the influence of classroom externalizing behaviors increases over time. If $\beta_{38}$ is positive and significant, this will indicate that for each unit representing the quality of the home environment above the mean score of the sample, a child's rate of change of adaptive functioning is expected to increase by the value of the coefficient. A positive and significant coefficient associated with mother-child interaction $\left(\beta_{39}\right)$ will indicate that for each unit above the sample mean on the NCAST score, an individual's rate of change of adaptive functioning is predicted to increase by the value of the coefficient. A negative and statistically significant associated with negative life events $\left(\beta_{310}\right)$ will indicate that for each negative life event that an individual experienced above the mean number of negative life events in the sample, that individual's rate of change of adaptive functioning is expected to decrease by the value of the coefficient.

The analysis will be repeated four times for the four different outcomes: communication, socialization, daily living skills, and total score of adaptive functioning.

Sample size considerations. Hierarchical linear modeling techniques are frequently employed to account for statistical nesting of individuals within groups, such as children within classrooms (Bickel, 2007). In these analyses, the classrooms are at the group level (level-2) and the individuals represent level-1 observations. Many methodologists concur that HLM analyses require at least 600 observations, consisting of at least 20 groups (Bickel, 2007). If the analysis consisted of children in classrooms, 600 observations in 20 groups corresponds with the following: 600 individuals, consisting of 20 classrooms with 30 individuals per classroom (Bickel, 2007). 
However, Bickel (2007) stipulates the following: "For any fixed sample size, results of multilevel analyses are improved by making the number of groups as large as possible, thereby diminishing the number of cases for each group" (Bickel, 2007, p. 282). Therefore, for the example noted above with 600 individuals in 20 classrooms, it would be preferable for these 600 individuals to be distributed into 50 classrooms.

For longitudinal HLM analyses, the individual is the group level, with multiple time points nested within single individuals (Bickel, 2007). The product of the number of time points and the number of individuals is the number of observations. For example, if there were 20 individuals and 30 time points, there would be 600 observations. According to Bickel (2007), it is optimal for the number of level-2 units (in this case, individuals) to be as large as possible. The sample for the proposed dissertation consists of five time points nested within 170 individuals. The 170 individuals represent the groups in multilevel analyses, and 170 groups far exceeds the recommended minimum (Bickel, 2007). 


\section{Chapter 4: Results}

This chapter first presents the descriptive statistics for each of the variables included in primary analyses. Then, bivariate correlations are presented. These bivariate correlations include the following: correlations between domains of adaptive functioning within and across time points, correlations between covariates and adaptive functioning outcomes, correlations between predictors and adaptive functioning outcomes, and correlations between each of the predictors and covariates. Then, results from multilevel analyses are presented for each of the four outcomes (socialization, communication, daily living skills, and total scores of adaptive functioning). Finally, results from follow-up analyses are presented. These follow-up analyses are intended to illuminate potential mechanisms that may account for the observed and unexpected positive relationships between negative life events and adaptive functioning outcomes.

\section{Preliminary Analyses}

Descriptive statistics. Tables 6 and 7 display descriptive statistics for the continuous and categorical variables. From Table 6, mean adaptive functioning raw scores appear to increase over time in each domain (socialization, communication, and daily living skills). Total adaptive functioning raw scores also appear to increase between ages 3 and 15. As Table 6 indicates, some participants had raw scores equal to 0. For each domain at each time point, and for the total score at each time point, the percentage of participants with raw scores equal to zero was equal to or less than 3.4. Multilevel analyses were completed with the inclusion of these scores equal to zero. Additionally, the raw scores equal to zero were recoded to equal 0.01 , and multilevel analyses were repeated. Results were consistent across the two sets of analyses. 
Table 6 also includes corresponding age equivalents for the mean scores in each domain at each time point. Age equivalent scores reflect levels of functioning expected of typically developing children. The age equivalents noted in Table 6 are substantially below the actual ages of study participants. This indicates that on average, participants were functioning below expected levels.

At each time point, mean levels of classroom externalizing behavior were below clinical levels for the respective measures of externalizing behavior (at ages 3 and 5 , scores of 70 and higher suggest "high levels of dysfunction"; at ages 8,10 , and 15, the clinical cut-off is equal to 60) (Achenbach \& Edelbrock, 1983; Kohn, 1988, p. 16). At ages 3 and $5,3.4 \%$ and $8.9 \%$ of participants had externalizing behavior scores that indicate "high-risk status with respect to social dysfunction in the classroom" (Kohn, 1988, p. 16). At ages 8,10 , and 15 the percentages of children with externalizing behavior scores above the clinical cut-off were equal to $39.8,32.6$, and 39.7. Table 6 also conveys that families experienced a wide range of negative life events; four families experienced no negative life events, and one family experienced 26 . On average, mothers had received more than a high school education by the time their children were three years old ( $M=13.88$ years of education).

Table 7 shows the percentage of children in substantially separate classrooms, as opposed to inclusive classrooms, at each time point. At ages 3, 5, 8, and 10, a child was considered to be in a substantially separate setting if at least $70 \%$ of the children in his or her class had an IEP. At age 15, a child was considered to be in substantially separate settings if his or her mother indicated that his or her school day was best described by any of the following: (1) day treatment or residential program, (2) special education with 
lunch separate from typically developing peers, (3) special education with lunch alongside typically developing peers, (4) special education plus time in employment and / or the community, (5) one to two hours of general education with the remainder of time in special education. Other response options included (6) one to two hours of special education with the remainder of time in general education, (7) general education with support, and (8) general education without support. From Table 7, approximately half of the children with DD were in substantially separate settings, as opposed to inclusive classrooms, at ages $5,8,10$, and 15 . At age 3 , the percentage of children in substantially separate settings was higher (76\%). Also from Table 7, approximately half of the children with DD are of each gender, and approximately one third fall into each of the three disability categories (Down syndrome, motor impairment, developmental delay of unknown etiology). Most families were of middle income; $51 \%$ of families reported incomes higher than $\$ 30,000$ between 1989 and 1991, when the median income in the United States was equal to $\$ 29,943$ (United States Census Bureau, 2012).

Bivariate correlations. Table 8 displays bivariate correlations between each domain of adaptive functioning at each time point (socialization, communication, and daily living skills at ages $3,5,8,10$, and 15). The domains of adaptive functioning are highly correlated with each other, both within and across time points. For example, at the age 3 time point, socialization scores are highly correlated with communication scores $(r=0.80, p<0.01)$ and daily living scores $(r=0.81, p<0.01)$. Also at age 3 , daily living scores and communication scores are highly correlated $(r=0.80, p<0.01)$. These substantial relationships between adaptive functioning domains at each time point persist over time; at age 15, correlation coefficients between adaptive functioning domains are 
highly significant and equal to or greater than 0.91 . Across time, scores on adaptive functioning outcomes are highly significantly related, but the magnitudes of the correlation coefficients are somewhat smaller. For example, age 3 socialization is related to age 15 socialization $(r=0.54, p<0.01)$, communication $(r=0.55, p<0.01)$, and daily living skills $(r=0.58, p<0.01)$.

Table 9 displays bivariate correlations between the covariates and each of the adaptive functioning outcomes (socialization, communication, daily living skills, and total scores) at each time point. Correlations between adaptive functioning outcomes and child and family demographic information will be discussed first. The gender of the child is not associated with any of the outcomes ( $p>0.05$ for each). Similarly, there are no significant relationships between family socioeconomic status and any of the adaptive functioning outcomes ( $p>0.05$ for each). There are relationships between children's disability diagnoses and adaptive functioning outcomes, such that having developmental delay of unknown etiology is significantly and positively associated with most of these outcomes, including socialization scores at ages 3,5 , and $10(r=0.20$ and $p<0.01$ at age $3, r=0.20$ and $p<0.01$ at age $5, r=0.15$ and $p<0.05$ at age 10 ); communication scores at ages $3(r=0.23, p<0.01), 5(r=0.22, p<0.01)$, and $8(r=0.19, p<0.05)$; and daily living scores at ages $3(r=0.31, p<0.01), 5(r=0.22, p<0.01), 8(r=0.25, p<0.01)$, and $10(r=0.23, p<0.01)$. Finally, there are significant relationships between developmental delay of unknown etiology and total scores of adaptive functioning (at age $3, r=0.27, p<0.01$; at age $5, r=0.23, p<0.01$; at age $8, r=0.21, p<0.01$, at age $10, r=$ $0.20, p<0.01)$. While children with developmental delay of unknown etiology score higher than other children on most adaptive functioning outcomes, there are no 
significant relationships between diagnosis of developmental delay of unknown etiology and adaptive functioning at age 15. This may suggest that the gap between these children and other children decreases over time.

There are significant relationships between diagnosis of Down syndrome and many of the adaptive functioning outcomes, such that diagnosis of Down syndrome is associated with lower adaptive functioning scores. At age 3, diagnosis of Down syndrome is significantly associated with lower communication scores $(r=-0.25, p<$ $0.01)$, daily living scores $(r=-0.20, p<0.01)$, and total scores $(r=-0.20, p<0.01)$. At age 5, diagnosis of Down syndrome is significantly associated with lower socialization $(r$ $=-0.19, p<0.05)$, communication $(r=-0.27, p<0.01)$, daily living $(r=-0.21, p<0.01)$, and total scores $(r=-0.23, p<0.01)$. At age 8 , diagnosis of Down syndrome is statistically significantly related to lower socialization $(r=-0.21, p<0.01)$, communication $(r=-0.24, p<0.01)$, daily living $(r=-0.24, p<0.01)$, and total adaptive functioning scores $(r=-0.24, p<0.01)$. Similar negative relationships exist between diagnosis of Down syndrome and adaptive functioning outcomes at age 10: for socialization, $r=-0.20, p<0.01$; for communication, $r=-0.18, p<0.05$; for daily living, $r=-0.22, p<0.01$; for total scores, $r=-0.21, p<0.01$. At age 15 , there are no significant relationships between diagnosis of Down syndrome and adaptive functioning, perhaps suggesting that the gap between children with Down syndrome and children with other DD decreases over time.

There are no significant relationships between diagnosis of motor impairment and scores on any of the adaptive functioning outcomes. Note that the variables representing disability diagnosis are coded differently in these preliminary analyses than in subsequent 
multilevel analyses; in the current preliminary analyses, children in each disability category are compared to all of the other children comprising the other two categories. In multilevel analyses, children with Down syndrome comprise the reference group, such that children with motor impairment and children with developmental delay of unknown etiology are compared only to children with Down syndrome. Children with motor impairment and children with developmental delay of unknown etiology are not compared to each other.

Regarding relationships between covariates representing classroom characteristics and dynamics and adaptive functioning outcomes, there are negative and significant relationships between classroom externalizing behaviors and many of the adaptive functioning outcomes. This suggests that children with higher levels of externalizing behaviors have lower levels of adaptive functioning. These relationships do not appear consistently over time; for example, at age 15 , there are no significant relationships between externalizing behaviors and adaptive functioning (both measured simultaneously). In contrast, at age 3 , there are significant and negative relationships between classroom externalizing behaviors and each adaptive functioning outcome at the same time point $(r=-0.22$ and $p<0.01$ for socialization, $r=-0.29$ and $p<0.01$ for communication, $r=-0.21$ and $p<0.01$ for daily living skills, $r=-0.26$ and $p<0.01$ for total adaptive functioning scores). There are also significant and negative relationships across some time points; classroom externalizing behaviors at age 10 are related to each of the age 3 adaptive functioning scores $(r=-0.18, p<0.05$ for socialization; $r=-0.20, p$ $<0.01$ for communication; $r=-0.16, p<0.05$ for daily living skills; $r=-0.19, p<0.05$ for total adaptive functioning scores). In every instance in which there is a statistically 
significant relationship between classroom externalizing behaviors and adaptive functioning, the relationship is negative, such that lower levels of externalizing behaviors are associated with higher levels of adaptive functioning.

There are also consistent negative relationships between classroom placement type and adaptive functioning scores. The negative direction of these relationships suggests that children in substantially separate settings, compared to children in inclusive settings, have lower levels of adaptive functioning. In every instance in which there is a significant relationship, it is negative. The variables representing classroom placement at ages 8,10 , and 15 are statistically significantly associated with every adaptive functioning outcome at every time point. Classroom placement at ages 3 and 5 are only inconsistently related to adaptive functioning outcomes. For example, there are no significant relationships between classroom placement type at age 5 and socialization, daily living skills, or total adaptive functioning scores at age 3 ( $p>0.05$ for each). The lack of significant relationships between age 3 adaptive functioning and age 5 classroom placement type may suggest that in the early grades, children with relatively low levels of adaptive functioning were still somewhat likely to be placed in inclusive settings. From Table 11, type of disability diagnosis is not related to classroom placement type at any time point.

Table 10 contains bivariate correlations between the predictors representing the early childhood home and family environment and each of the adaptive functioning outcomes. Child evocative effects are not associated with most of the adaptive functioning outcomes, but there are significant relationships between child evocative effects and socialization and total scores at age $15(r=-0.18$ and $p<0.05$ for 
socialization; $r=-0.15$ and $p<0.05$ for total scores). The negative direction of these relationships suggests that more stressful child evocative effects in early childhood is related to lower levels of adaptive functioning at age 15 .

Higher quality mother-child interaction (measured at age 3 ) is statistically significantly associated with each of the adaptive functioning outcomes at ages $3,5,8$, and 15, such that higher quality interaction is associated with higher levels of socialization $(r=0.15$ and $p<0.05$ at age $3 ; r=0.22$ and $p<0.01$ at age $5 ; r=0.20$ and $p<0.05$ at age $8 ; r=0.22$ and $p<0.01$ at age 15$)$, communication $(r=0.16$ and $p<$ 0.05 at age $3 ; r=0.22$ and $p<0.01$ at age $5, r=0.16$ and $p<0.05$ at age $8 ; r=0.21$ and $p<0.01$ at age 15), and daily living skills $(r=0.20$ and $p<0.01$ at age $3 ; r=0.19$ and $p$ $<0.05$ at age $5 ; r=0.18$ and $p<0.05$ at age $8 ; r=0.17$ and $p<0.05$ at age 15$)$, as well as higher total scores of adaptive functioning $(r=0.18$ and $p<0.05$ at age $3 ; r=0.22$ and $p<0.01$ at age 5; $r=0.18$ and $p<0.05$ at age $8 ; r=0.20$ and $p<0.01$ at age 15). Of note is the lack of significant relationships between quality of mother-child interaction and any of the adaptive functioning outcomes at age 10 .

Higher quality of the home environment is statistically significantly related to higher levels of adaptive functioning at ages 3 and 15. Specifically, there are significant relationships between the quality of the early childhood home environment and socialization $(r=0.18$ and $p<0.05$ at age $3 ; r=0.19$ and $p<0.05$ at age 15$)$, communication $(r=0.20$ and $p<0.05$ at age $3 ; r=0.17$ and $p<0.05$ at age 15$)$, and total scores of adaptive functioning $(r=0.18$ and $p<0.05$ at age $3 ; r=0.17$ and $p<0.05$ at age 15). There are no significant relationships between quality of the early childhood home environment and adaptive functioning scores at ages 8 and 10 . There are also no 
significant relationships between quality of the home environment and levels of daily living skills.

Finally, negative life events are statistically significantly and positively associated with adaptive functioning outcomes, indicating that children who experienced more negative life events display higher levels of adaptive functioning. At ages 3 and 5, there are significant and positive relationships between negative life events and socialization $(r$ $=0.17$ and $p<0.05$ at age $3 ; r=0.16$ and $p<0.05$ at age 5 ). At age 8 , negative life events are positively and significantly associated with communication $(r=0.18, p<$ 0.05 ), daily living skills $(r=0.17, p<0.05)$, and total scores of adaptive functioning ( $r=$ $0.17, p<0.05)$. At age 10, there are positive and significant relationships between negative life events and each of the adaptive functioning outcomes, including socialization $(r=0.16, p<0.05)$, communication $(r=0.20, p<0.01)$, daily living skills $(r=0.19, p<0.05)$, and total scores $(r=0.19 p<0.05)$. There are no significant relationships at age 15. Note that the direction of each significant relationship is opposite of the hypothesized direction; this will be discussed further in the subsequent discussion chapter.

Finally, Table 11 shows the bivariate correlations between each of the predictors and covariates. For the child and family demographic variables, notable findings include unique characteristics of families that include a child with Down syndrome. Having Down syndrome is associated with higher levels of family socioeconomic status $(r=$ $0.22, p<0.01)$, higher levels of classroom externalizing behavior at ages 8 and $10(r=$ $0.16, p<0.05$ for age $8 ; r=0.26, p<0.01$ for age 10 ), lower levels of parent-reported stress stemming from child evocative effects $(r=-0.23, p<0.01)$, higher quality of the 
home environment $(r=0.14, p<0.01)$, and the experience of fewer negative life events $(r=-0.09, p<0.01)$. There are also relationships between family socioeconomic status and many of the early childhood home and family indicators. Higher socioeconomic status is related to higher quality mother-child interaction $(r=0.23, p<0.01)$, higher quality of the home environment $(r=0.45, p<0.01)$, and the experience of fewer negative life events $(r=-0.33, p<0.01)$.

There are also several significant relationships between classroom characteristics and dynamics and indicators of the early childhood home and family environment. Placement in substantially separate settings, compared to placement in inclusive settings, is related to lower levels of early childhood mother-child interaction. However, this relationship is only significant for the age 8 classroom placement $(r=-0.18, p<0.05)$. Placement in a substantially separate setting at age 10 is significantly related to lower quality of the home environment $(r=-0.17, p<0.05)$. Also, higher levels of child externalizing behaviors at ages 3,5 , and 8 are related to lower levels of mother-child interaction $(r=-0.33$ and $p<0.01$ at age $3 ; r=-0.24$ and $p<0.01$ at age $5 ; r=-0.19$ and $p<0.01$ at age 8 ). Classroom externalizing behaviors at ages 3, 5, and 15 are related to lower quality of the home environment $(r=-0.33$ and $p<0.01$ at age $3 ; r=-0.21$ and $p<$ 0.01 at age $5 ; r=-0.19$ and $p<0.05$ at age 15$)$, and classroom externalizing behaviors at age 3 are significantly and positively associated with the experience of more negative life events $(r=0.19, p<0.05)$, suggesting that more negative life events are associated with higher levels of externalizing behaviors. At most time points, the two classroom covariates (classroom placement type and classroom levels of externalizing behaviors) are not statistically significantly correlated with each other. However, at age 10, there is 
a significant relationship between classroom placement and levels of classroom

externalizing behaviors, such that children in substantially separate settings exhibit higher levels of externalizing behaviors than children in inclusive settings $(r=0.26, p<0.01)$.

There are also significant relationships between many of the early childhood home and family indicators. Lower levels of parent-reported child evocative effects during early childhood is associated with higher quality mother-child interaction $(r=$ $0.11, p<0.01)$, higher quality of the home environment $(r=-0.09, p<0.05)$, and the experience of fewer negative life events $(r=0.17, p<0.01)$. Higher quality mother-child interaction is associated with higher quality of the home environment $(r=0.37, p<0.01)$ and the experience of fewer negative life events $(r=-0.14, p<0.01)$. There is also a statistically significant relationship between the quality of the home environment and the experience of negative life events, such that higher quality is related to fewer negative events $(r=-0.19, p<0.05)$.

\section{Primary Analyses}

\section{Multilevel models predicting socialization.}

Unconditional model. Table 12 contains results from the multilevel equations predicting levels of classroom-based socialization skills. The results from the unconditional model provide support for hypotheses 1 and 2; socialization raw scores increase over time, and there is significant variability between people with regard to initial status (socialization scores at age 3 ) and rates of change. The coefficient associated with age was positive (0.40) and statistically significantly different from zero $(p<0.01)$, indicating that as children's ages increased, so did their socialization scores. The value of $\sigma^{2}{ }_{0}$ represents variability in initial status. It was equal to 95.00 and was 
statistically significantly different from zero $(p<0.01)$, indicating significant variability in intercept. The value of $\sigma^{2}{ }_{1}$ was equal to 0.01 and represents variability in the slope. Since this was statistically significantly different from zero $(p<0.01)$, there was significant variability in children's rate of growth in socialization skills.

The unconditional model also provided an estimate of the intercept, or the average socialization score at age 3 . This estimate was equal to 22.24 , and was statistically significantly different from zero $(p<0.01)$. Finally, results from this unconditional model indicated that the squared age term was not statistically significant $(p>0.05)$. Therefore, the squared age term was excluded from subsequent equations predicting socialization. Figure 1 shows the trajectory of growth in classroom-based socialization for the sample, with the age of the child as the only predictor.

Model with covariates. Initial multilevel analyses indicated that the gender of the child and the socioeconomic status of the family were not significant predictors of initial status or growth of classroom-based socialization ( $p>0.05$ for each). Therefore, these variables were excluded from the models presented. Also, since results from the unconditional model indicated that the squared age term was not a significant predictor, this variable was also excluded.

Model 2 in Table 12 displays results from multilevel equations including significant covariates, each of which will be described in turn. The intercept was equal to 20.88 , and this value was statistically significantly different than zero $(p<0.01)$. Since children with Down syndrome comprised the reference group, this value indicated that children with Down syndrome had an average socialization raw score of 20.88 at age 3, assuming mean levels of classroom externalizing behaviors and placement in an inclusive 
setting. The coefficient representing developmental delay of unknown etiology was positive and significant $(\beta=8.82, p<0.01)$. This indicated that children with developmental delays of unknown etiology had an average initial status that was equal to 8.82 units higher than the average initial status of children with Down syndrome. Therefore, the estimated average initial status of children with developmental delays of unknown etiology was equal to $29.70(20.88+8.82)$. The coefficient representing motor impairment was also positive $(\beta=3.94)$ but it was not statistically significantly different than zero $(p>0.05)$. This indicated that the initial status of children with motor impairment was not statistically significantly different than the initial status of children with Down syndrome. In order to test for the possibility that different disability groups exhibit different rates of change in socialization raw scores, interactions were tested between the variables representing disability diagnostic categories and the variable representing the age of the child. These interaction terms were not significant and were therefore excluded from the model.

Both of the variables representing classroom characteristics were significant predictors of socialization raw scores (initial status). The coefficient representing placement in a substantially separate setting was equal to -4.81 , and was statistically significantly different than zero $(p<0.01)$. Children in substantially separate settings, compared to children in inclusive settings, scored on average 4.81 units lower on the socialization measure at age 3 . The variable representing classroom externalizing behaviors was also negative and statistically significantly different than zero ( $\beta=-3.46, p$ $<0.01$ ). For every unit higher that a child scored on the measure of classroom externalizing behavior, that child was predicted to score 3.46 points lower on 
socialization measured at age 3. Placement type (substantially separate versus inclusive) and level of classroom externalizing behavior problems were also tested as predictors of the slope. However, neither placement type nor externalizing behaviors were significant predictors of the slope, and therefore these predictors of the slope were excluded from the model.

The covariance parameters of Model 2 (displayed in Table 12) indicated that there remained significant unexplained variability in initial status (socialization raw score at age $\left.3 ; \sigma^{2}=105.21 ; p<0.01\right)$ and rate of change $\left(\sigma^{2}{ }_{1}=0.02, p<0.01\right)$. Model 3 was constructed to predict this variability with variables representing the early childhood home and family environment. Finally, the fit statistics for Model 2 represented lower values than the fit statistics for Model 1 (-2 Log Likelihood $=7298.50$ for Model 1 and 7124 for Model 2; $\mathrm{AIC}=7312.50$ for Model 1 and 7142.32 for Model 2; $\mathrm{BIC}=7346.04$ for Model 1 and 7142.52 for Model 2). This indicated that the inclusion of covariates (including variables representing child diagnostic category and classroom characteristics and dynamics) improved the fit of the model.

Model with predictors. Model 3 in Table 12 displays results from the final multilevel models predicting socialization raw scores. Model 3 included indicators of the early childhood home and family environment. It tested the hypothesis that early childhood home and family indicators would predict classroom adaptive functioning, including adaptive functioning initial status and rate of change (hypothesis 3). The intercept was equal to 21.01 and was statistically significantly different than zero ( $p$ $<0.01)$. The value of the intercept (21.01) represents the average socialization raw score at age 3, after taking into account other variables in the model. Specifically, the intercept 
represents the average socialization raw score at age 3 for children with Down syndrome in inclusive settings, with mean levels of classroom behavior problems, and mean scores representing both negative life events and mother-child interaction. The coefficient associated with age was positive and significant $(\beta=0.39, p<0.01)$. For each year of age, children were predicted to show gains in socialization raw scores equal to 0.39 units. This estimate pertained to children across all diagnostic categories, all classroom placement types, all levels of externalizing behaviors, all numbers of negative life events, and for children with mean levels of mother-child interaction.

Diagnosis of developmental delay of unknown etiology was a positive and significant predictor of the intercept $(\beta=8.32, p<0.01)$, indicating that children with developmental delay of unknown etiology had an initial socialization raw score that was 8.32 units higher than that of children with Down syndrome. The coefficient associated with motor impairment was not significant $(p>0.05)$, indicating no difference in initial status between children with Down syndrome and children with motor impairment. As in Model 2, classroom placement type and level of classroom externalizing behavior were significant predictors of socialization raw scores at age $3(\beta=-4.68, p<0.01$ for classroom placement type; $\beta=-3.54, p<0.01$ for level of externalizing behavior). Children in substantially separate settings, compared to children in inclusive settings, had estimated age 3 socialization raw scores that were 4.68 units lower. For each unit higher that children scored on the measure of externalizing behavior, their predicted socialization raw scores decreased by 3.54 units. Neither of these variables were significant predictors of growth in socialization raw scores. 
As a test of hypothesis 3, Model 3 also included the predictors representing the early childhood home and family environment. Child evocative effects and the quality of the home environment were not significant predictors of the slope or the intercept, and thus were excluded from the model. The number of negative life events experienced by the family during early childhood was a statistically significant predictor of socialization raw scores at age $3(\beta=0.65, p<0.01)$. Since this coefficient was positive, the direction of this relationship was opposite to the hypothesized direction; children who experienced more negative life events had higher socialization raw scores at age 3 than children who experienced fewer such events. For each event that a child experienced above the mean number of events for the sample, that child's socialization raw score was predicted to increase by 0.65 units. The fact that the direction of this relationship is positive (and opposite of the hypothesized direction) will be discussed in further detail in the discussion chapter. The number of negative life events experienced by the family was not a significant predictor of growth in socialization raw scores.

Mother-child interaction was the final predictor in Model 3. It was not a significant predictor of the initial status of socialization raw scores, but it was a significant predictor of growth in this domain $(\beta=0.004, p<0.05)$. Since the coefficient was positive, higher quality mother-child interaction was associated with more rapid growth. The following equation represents the predicted values of socialization raw scores, and includes all significant predictors and covariates:

$$
\begin{aligned}
& Y_{t i}=21.01+(8.32)\left(D D_{i}\right)+(-3.54)\left(E X T_{t i}\right)+ \\
& (-4.68)\left(C L A S S_{t i}\right)+(0.65)\left(N L E_{i}-\overline{N L E} \bullet\right)+(0.39)\left(A G E_{t i}\right)+ \\
& +(0.004)\left[A G E_{t i} *\left(M C I_{i}-\overline{M C I} \cdot\right)\right] \\
& +r_{0 i}+r_{1 i}\left(A G E_{t i}\right)+e_{t i}
\end{aligned}
$$


, where DD represents developmental delay of unknown etiology, EXT represents levels of classroom externalizing behaviors, CLASS represents classroom placement type (substantially separate versus inclusive), NLE represents the number of negative life events experienced by the family during early childhood, AGE represents the age of the child, and MCI represents the quality of mother-child interaction during early childhood.

Figures 5-8 show trajectories for children according to quality of mother childinteraction (Figures 5 and 6) and numbers of negative life events (Figures 7 and 8) assuming placement in inclusive settings and mean levels of classroom externalizing problems. When there are significant differences between disability categories, the figures display trajectories separately for each disability category.

Tables 17-19 show predicted values of socialization raw scores based on high and low quality of early childhood mother-child interaction, as well as high and low numbers of negative life events during early childhood. For both mother-child interaction and negative life events, "high" and "low" values correspond with values equal to one standard deviation above and below the sample mean. The three tables (17-19) display the predicted values for the three disability categories (Table 17 contains predicted values for children with Down syndrome, Table 18 contains predicted values for children with motor impairment, and Table 19 contains predicted values for children with developmental delay of unknown etiology.) As these tables indicate, at age 15, children with high levels of mother-child interaction have socialization raw scores that are approximately 0.5 units higher than children with low levels of mother-child interaction. The corresponding age equivalent scores indicate that this difference is equal to approximately one month of growth in typically developing children. Also from Tables 
17-19, at each time point, children with high numbers of negative life events have raw scores that are approximately 6 units greater than children with low numbers of negative life events. The age equivalent scores indicate that this discrepancy corresponds with approximately 5 months of growth, based on growth expected of typically developing children.

The covariance parameters of Model 3 (displayed in Table 12) indicated that there remained significant unexplained variability in initial status $\left(\sigma_{0}^{2}=94.71 ; p<0.01\right)$ and rate of change $\left(\sigma^{2}=0.02, p<0.01\right)$. The addition of early childhood home and family indicators explained $9.98 \%$ of the variability in initial status that remained after accounting for covariates; the estimate of the variability in rate of change did not change with the addition of early childhood home and family indicators. The fit statistics (also in Table 12) partially supported the suggestion that Model 3 represented a better fit than Model 2. The values of the AIC and -2 Log Likelihood decreased ( -2 Log Likelihood $=$ 7124.32 in Model 2 and 7107.52 in Model 3; AIC $=7142.32$ in Model 2 and 7131.52 in Model 3) and this decrease suggested improved fit. However, the value of the BIC increased (7142.52 in Model 2, 7188.75 in Model 3). The model was re-run without the two insignificant predictors of the intercept (diagnosis of motor impairment and quality of mother-child interaction) but the BIC for Model 3 remained higher than that for Model 2 (7142.52 in Model 2, 7180.49 for Model 3). These variables were therefore retained, since model fit did not demonstrate substantial improvement, and it is generally recommended to retain variables representing insignificant main effects, if they are included in significant interaction terms (Chandler \& Scott, 2011). 
In light of the decreases in the AIC and the -2 Log Likelihood, and despite the increase in the BIC, Model 3 was selected as the final model (Stock \& Watson, 2007). This is in line with recommendations from Stock and Watson (2007) to select the model suggested by the AIC. Usually, the model recommended by the AIC will include more predictors than that recommended by the BIC, since the BIC tends to favor the more parsimonious model (Stock \& Watson, 2007). The additional predictors in Model 3 (those that aren't included in Model 2, including negative life events and mother-child interaction) were significant, and therefore were retained (Stock \& Watson, 2007).

\section{Multilevel models predicting communication.}

Unconditional model. The results from the unconditional model predicting communication are displayed in Table 13. The intercept was equal to 24.60, indicating that 24.60 was the average raw score of communication at age 3 . This value was statistically significantly different than zero $(p<0.01)$. With regard to hypothesis 1 , the coefficient representing age was positive $(\beta=0.71)$ and statistically significantly different than zero ( $p<0.01$ ), indicating support for the hypothesis that communication raw scores increase over time. With regard to hypothesis 2 , the values of $\sigma_{0}^{2}$ and $\sigma^{2}{ }_{1}$ ( 225.51 and 0.02 , respectively) were both statistically significantly different than zero ( $p$ $<0.05)$. This indicated support for the hypothesis that there was variability between people with regard to both initial status and rate of change. Finally, the coefficient representing the squared age term was negative and statistically significantly different than zero $(\beta=-0.002, p<0.01)$. The fact that the coefficient was negative indicated a decrease in rate of growth over time; as children grew older, their growth slowed. The value of the coefficient, however, was quite small. Figure 2 shows the growth trajectory 
of classroom-based communication skills for the entire sample. It demonstrates that skills increased over time, with a very slight decrease in rate of change.

Model with covariates. Model 2 in Table 13 displays results from multilevel equations predicting classroom-based communication skills, with covariates representing child and family demographic information, as well as covariates representing classroom characteristics and dynamics. The value of the intercept was equal to 21.65 and was statistically significantly different than zero $(p<0.01)$. Taking into account all of the other variables in the model, this value indicated that children with Down syndrome in inclusive settings with mean levels of classroom-based externalizing behavior problems were estimated to have raw communication scores equal to 21.65 at age 3 . The coefficient associated with age was positive and significant $(\beta=0.56, p<0.01)$. This positive value of the coefficient indicated that children's communication raw scores increased over time. The value of the coefficient associated with the squared age term was not significant $(p>0.05)$. However, as described below, there were significant interactions between child characteristics and the squared age term.

Gender of the child with a disability and family socioeconomic status were initially included as covariates representing child and family demographic information. However, these variables were not significant predictors of either the intercept or the slope, and were therefore excluded from the model. Diagnosis of developmental disability of unknown etiology was a positive and significant predictor of the intercept ( $\beta=12.32, p<0.01)$, indicating that children with developmental delay of unknown etiology were estimated to score 12.32 points higher than children with Down syndrome on the measure of communication at age 3. Diagnosis of developmental disability of 
unknown etiology was a significant predictor of the linear slope $(\beta=0.20, p<0.05)$. The positive value of this coefficient indicated that children with developmental delay of unknown etiology displayed more rapid growth in classroom-based communication than children with Down syndrome. The value of the coefficient representing the interaction between developmental delay of unknown etiology and the squared age term was negative and significant $(\beta=-0.002, p<0.01)$. The negative value of this coefficient indicated that for children with developmental delay of unknown etiology, the pace of growth slowed over time. The value of the coefficient was very small, however, and likely did not indicate a ceiling effect.

Diagnosis of motor impairment was not a significant predictor of the intercept ( $\beta$ $=6.75, p>0.05)$. This indicated that children with motor impairment did not differ from children with Down syndrome in classroom-based communication skills at age 3. However, diagnosis of motor impairment was a significant predictor of the slope $(\beta=$ $0.17, p<0.05)$. The positive value of this coefficient indicated that children with motor impairment displayed more rapid growth in classroom-based communication skills than children with Down syndrome. The coefficient representing the interaction between motor impairment and the squared age term was small in magnitude, yet it was negative and significant $(\beta=-0.002, p<0.01)$. The negative value of this term indicated that children with motor impairment exhibited a decline in rate of change over time.

Both of the variables representing classroom characteristics and dynamics were significant predictors of initial status, or communication skills at age 3 . Children in substantially separate settings, compared to children in inclusive settings, scored on average 5.24 points lower on the communication measure at age $3(\beta=-5.24, p<0.01)$. 
Higher levels of externalizing behavior problems were statistically significantly associated with lower levels of communication skills at age $3(\beta=-2.21, p<0.01)$. The variables representing externalizing behavior problems and placement in a substantially separate setting were not significant predictors of the linear or the non-linear slope.

The covariance parameters indicated that there was still unexplained significant variability in both communication initial status $\left(\sigma_{0}^{2}=225.57, p<0.01\right)$ and rate of change $\left(\sigma^{2}{ }_{1}=0.03, p<0.01\right)$. Model 3 was constructed to predict this variability with indicators of the early childhood home and family environment.

Finally, the fit statistics (listed in Table 13) indicated that Model 2 fits the data better than Model 1. The values of the -2 Log Likelihood, the AIC, and the BIC were lower for Model 2 than for Model 1 (-2 Log Likelihood $=7667.10$ for Model 1, and 7462.99 for Model 2; $\mathrm{AIC}=7681.10$ for Model 1, and 7490.99 for Model 2; $\mathrm{BIC}=$ 7714.64 for Model 1, and 7557.75 for Model 2).

Model with predictors. Model 3 in Table 13 shows the results from the final multilevel models predicting communication raw scores over time. This model included the covariates from Model 2. Also, in order to test the hypothesis that indicators of the early childhood home and family environment predict classroom-based trajectories of communication skills, this model also included indicators of the early childhood home and family environment. The intercept was equal to 21.69 , and this was statistically significantly different than zero $(p<0.01)$. This value corresponded to the average communication raw score at age 3 , taking into account the other variable in the model. This estimate of the intercept pertains to children with Down syndrome, in inclusive placements, with mean levels of classroom externalizing behavior problems, mean levels 
of mother-child interaction, and the mean number of negative life events. The coefficient associated with age was positive and significant $(\beta=0.56, p<0.01)$, indicating that children with Down syndrome exhibited growth in communication raw scores at a rate of 0.56 units per year. As described below, there were significant interactions between disability diagnosis and the squared and linear age terms, indicating that children in other diagnostic groups exhibited different rates of change. The coefficient associated with the squared age term was not significant $(p>0.05)$.

Significant predictors of the intercept included diagnosis of developmental delay of unknown etiology, $(\beta=11.90, p<0.01)$, indicating that children with this diagnosis had age 3 communication raw scores that were 11.90 units higher than communication raw scores of children with Down syndrome. The coefficient associated with motor impairment was also positive and significant $(\beta=6.75, p<0.05)$, indicating that children with this diagnosis had age 3 communication raw scores equal to 6.75 units higher than the raw scores of children with Down syndrome. The coefficients associated with classroom placement type and classroom externalizing behaviors were both negative and significant $(\beta=-5.13$ and -2.13 respectively, $p<0.01$ for each). Children in substantially separate settings had age 3 communication raw scores that were 5.13 units lower than the raw scores of children in inclusive placements. In relation to behavior problems, for each unit higher that a child scored on the measure of externalizing behavior, that child's age 3 communication raw score was expected to decrease by 2.13 units.

Regarding hypothesis 3, two variables representing the early childhood home and family environment were significant predictors of age 3 communication raw scores. The coefficient associated with negative life events was equal to $0.73(p<0.05)$, indicating 
that for each additional negative life event that a child experienced above the mean number of negative life events in the sample, that child's age 3 communication raw score was expected to increase by 0.73 . The positive direction of this relationship is opposite from the hypothesized negative direction; this will be discussed in greater detail in later sections. The coefficient associated with mother-child interaction was positive and significant $(\beta=0.59, p<0.01)$. For each unit increase in the measure of mother-child interaction, children's age 3 communication raw scores were predicted to increase by 0.59 units. The coefficients associated with the home environment and child evocative effects in the home were not significant.

None of the variables representing the early childhood home and family environment were significant predictors of rate of change of communication raw scores. Diagnosis of motor impairment and diagnosis of developmental delay of unknown etiology were both significant predictors of the linear and squared age terms. The magnitude, direction, and interpretation of these coefficients were identical to the magnitude, direction, and interpretation of the respective coefficients in Model 2. Figures 9-14 show the estimated trajectories in communication raw scores for children with high and low levels of mother-child interaction quality (Figures 9-11), and for children who had experienced high and low numbers of negative life events (Figures 1214). There are separate figures corresponding to individual diagnostic categories. Tables 17-19 contain predicted values of communication raw scores for children with high and low quality early childhood mother-child interaction, as well as for children who experienced high and low numbers of negative life events during early childhood. These "high" and "low" values correspond with the values equal to one standard deviation 
above and below the sample means. Tables 17,18 , and 19 contain predicted values for children with Down syndrome, motor impairment, and developmental delay of unknown etiology, respectively. From these tables, at each time point, the effect associated with high quality mother-child interaction is equal to approximately 7 raw score units. The corresponding age equivalents indicate that this corresponds with approximately 3-4 months of growth that would be expected of typically developing children. Also from these tables, at each time point, children who experienced high numbers of negative life events have communication raw scores that are approximately 7 units higher than children who experienced low numbers of negative life events. From the age equivalent scores listed in Tables 17-19, this corresponds with approximately 3-5 months of growth that would be expected of typically developing children.

The final equation representing estimated communication raw scores is as follows:

$$
\begin{aligned}
& Y_{t i}=21.69+(11.90)\left(D D_{i}\right)+(6.75)\left(M I_{i}\right)+(-2.13)\left(E X T_{t i}\right)+ \\
& (-5.13)\left(C L A S S_{t i}\right)+(0.73)\left(N L E_{i}-\overline{N L E} \cdot\right)+(0.59)\left(M C I_{i}-\overline{M C I} \bullet\right)+(0.56)\left(A G E_{t i}\right)+ \\
& +(0.20)\left(A G E_{t i} * D D_{i}\right)+(0.17)\left(A G E_{t i} * M I_{i}\right)-(0.002)\left[A G E S Q_{t i} * D D_{i}\right]-(0.002)\left[A G E S Q_{t i} * M I_{i}\right] \\
& +r_{0 i}+r_{1 i}\left(A G E_{t i}\right)+e_{t i}
\end{aligned}
$$

, where DD represents diagnosis of developmental delay of unknown etiology, MI represents diagnosis of motor impairment, EXT represents levels of classroom externalizing behaviors, CLASS represents classroom placement type (substantially separate versus inclusive), NLE represents the number of negative life events experienced by the family during early childhood, MCI represents quality of mother-child interaction during early childhood, AGE represents the age of the child, and AGESQ represents the squared age term. 
The covariance parameters (also in Table 13) indicated that there was still unexplained and significant variability in both the intercept and the slope (for intercept, $\sigma^{2}{ }_{0}=206.47, p<0.01$; for slope, $\left.\sigma^{2}{ }_{1}=0.03, p<0.01\right)$. The addition of the variables representing the early childhood home and family environment explained $8.47 \%$ of the variability in initial status; it explained a negligible amount of variability in slope, since early childhood home and family indicators were not significant predictors of the slope. The values of the -2 Log Likelihood and the AIC decreased from Model 2 to Model 3, indicating an improvement in model fit (-2 Log Likelihood $=7462.99$ in Model 2 and 7451.13 in Model 3; AIC $=7490.99$ in Model 2, and 7483.13 in Model 3). However, the value of the BIC increased slightly, indicating less adequate model fit (7557.75 in Model 2, 7559.43 in Model 3). However, all predictors in the current model, with the exception of the squared age term, were significant. Therefore, each predictor was retained, in order to include significant predictors (Stock \& Watson, 2007) and variables included in interaction terms (Chandler \& Scott, 2011).

\section{Multilevel models predicting daily living skills.}

Unconditional model. Table 14 displays results from the unconditional model predicting growth in classroom-based daily living skills (Model 1). The value of the intercept was equal to 22.61 and was statistically significantly different than zero $(p<$ 0.01 ) indicating that the average daily living raw score at age 3 was equal to 22.61. The coefficient representing age was positive and statistically significantly different than zero $(\beta=1.29, p<0.01)$, indicating support for the hypothesis that raw scores of classroombased daily living skills increase over time (hypothesis 1). The coefficient representing the squared age term was negative and statistically significantly different than zero $(\beta=-$ 
$0.01, p<0.01)$, suggesting that rate of growth in daily living skills decreased over time. Figure 3 displays the trajectory of growth of classroom-based daily living skills for the entire sample. This figure illustrates an overall pattern of positive growth over time, with a very small decline in rate of change.

With regard to variability between people in terms of initial status and rate of change (hypothesis 2), the unconditional model indicated that this variability did exist. The value of $\sigma^{2}{ }_{0}$ represents variability in initial status; it was equal to 349.03 and was statistically significantly different from zero $(p<0.01)$. The value $\sigma^{2}{ }_{1}$ represents variability in rate of change. It was equal to 0.05 and was also statistically significantly different than zero.

Model with covariates. Model 2 in Table 14 displays results from the multilevel equations predicting daily living skills with covariates including child and family demographic information and classroom dynamics and characteristics. Results from this model provided an estimate of the intercept equal to 24.24 , and this was statistically significantly different than zero $(p<0.01)$. After taking account other variables in the model, the estimate of the intercept indicated that on average, children with Down syndrome in inclusive settings with mean levels of externalizing behavior problems had a daily living raw score equal to 24.24 at age 3 . The coefficient associated with age was positive and statistically significant $(\beta=0.98, p<0.01)$, indicating that for each year of age, children's daily living raw scores were expected to increase by 0.98 . Finally, the coefficient associated with the age squared term was negative and statistically significant, indicating that children's rate of growth was predicted to decline over time $(\beta=-0.003, p$ $<0.01)$. 
Model 2 also included covariates representing child and family demographic information and classroom characteristics and dynamics. Child's gender and family socioeconomic status were not significant predictors of either the intercept or the slope, and therefore these variables were excluded from Model 2. Diagnosis of developmental delay of unknown etiology was a significant predictor of the intercept $(\beta=10.84, p<$ $0.01)$, the linear slope $(\beta=0.47, p<0.01)$, and the non-linear slope $(\beta=-0.003, p<0.01)$. These results indicated that compared to children with Down syndrome, children with developmental delay of unknown etiology had an initial status that was higher by 10.84 units. Since the coefficient associated with the linear slope was positive, children with developmental delay of unknown etiology had a faster rate of growth than children with Down syndrome. The coefficient representing the interaction term between developmental delay of unknown etiology and the non-linear term was negative, indicating that the decline in the rate of change (or the lessening of the slope over time) was more pronounced for children with developmental delay of unknown etiology than for children with Down syndrome. This lessening of the slope was very slight; at each time point children with developmental delay of unknown etiology had higher raw scores of daily living skills than children with Down syndrome.

Children with motor impairment did not differ from children with Down syndrome in their initial status of daily living raw scores $(\beta=2.47, p>0.05)$. However, diagnosis of motor impairment was a significant predictor of the linear slope $(\beta=0.32, p<$ 0.01). The value of the coefficient was positive, and this indicated that children with motor impairment exhibited more rapid growth in classroom-based daily living raw scores than children with Down syndrome. The coefficient representing the interaction 
between motor impairment and the squared age term was negative $(\beta=-0.003, p<0.01)$. This indicated that over time, growth slows more for children with motor impairment than for children with Down syndrome. However, at each time point, children with motor impairment displayed higher levels of classroom-based daily living raw scores than children with Down syndrome.

The variables representing classroom characteristics and dynamics were both statistically significant predictors of initial status. The variable indicating whether the child was in a substantially separate setting had a coefficient that was negative and significant $(\beta=-8.86, p<0.01)$. This indicated that compared to children in inclusive settings, the initial status of children in substantially separate settings was 8.86 units lower. The coefficient representing classroom-based externalizing behaviors was also negative and statistically significant $(\beta=-3.69, p<0.01)$. For each unit higher than children scored on the measure of externalizing behavior, they were predicted to score 3.69 units lower on the measure of daily living skills at age 3 . Neither classroom placement type (substantially separate versus inclusive setting) nor level of externalizing behavior problems were significant predictors of the linear or non-linear slope, and therefore these terms were excluded from the model.

The covariance parameters indicated that there was still significant unexplained variability in initial status $\left(\sigma^{2}=246.47, p<0.01\right)$ and slope $\left(\sigma^{2}{ }_{1}=0.05, p<0.01\right)$. The inclusion of predictors representing the early childhood home and family environment sought to explain this variability. Finally, the fit statistics indicated that Model 2 represented a better fit to the data than Model 1. The value of the AIC decreased from Model 1 to Model 2 (8238.80 for Model 1, and 7989.84 for Model 2). The values of the 
BIC followed a similar pattern (8267.54 for Model 1, and 8056.60 for Model 2), as did the values of the -2 Log Likelihood (8226.80 for Model 1, 7961.84 for Model 2).

Model with predictors. Model 3 in Table 14 displays results from the final multilevel models predicting daily living raw scores. This model included predictors that represented the early childhood home and family environment, and therefore was a test of the hypothesis (hypothesis 3) that the early childhood home and family environment would predict development in this domain. The estimated value of the intercept was equal to 24.30 , and this was statistically significantly different than zero $(p<0.01)$. Taking into account the other variables in the model, this value (24.30) represented the estimated daily living raw score at age 3 for children with Down syndrome, in inclusive settings, with mean levels of classroom externalizing behaviors, the mean number of negative life events, and mean levels of mother-child interaction quality. Significant predictors of the intercept included diagnosis of developmental delay of unknown etiology $(\beta=10.26, p<0.05)$, classroom placement type $(\beta=-8.65, p<0.01)$, classroom externalizing behaviors $(\beta=-3.56, p<0.01)$, negative life events $(\beta=0.90, p<0.05)$, and quality of mother-child interaction $(\beta=0.64, p<0.05)$. The significance of negative life events and mother-child interaction indicated support for the hypothesized relationship between the early childhood home and family environment and trajectories of classroom-based adaptive functioning. Child evocative effects within the home and the quality of the home environment were not significant predictors of daily living raw scores (slope or intercept) and were therefore excluded from the model.

Children with developmental delay of unknown etiology had predicted daily living raw scores at age 3 that were 10.26 units higher than scores of children with Down 
syndrome; there was no difference in daily living initial status between children with Down syndrome and children with motor impairment. Children in inclusive settings had initial daily living scores 8.65 units higher than children in substantially separate settings, and for each unit higher that a child scored on the measure of externalizing behaviors, that child's estimated age 3 daily living raw score decreased by 3.56 units. Regarding the predictors representing the early childhood home and family environment, for each additional negative life event that a child experienced, that child's daily living raw score at age 3 was expected to increase by 0.90 units. The relationship of this direction is opposite of the hypothesized direction, and this will be discussed in greater detail later on. Finally, for each unit higher that a mother-child dyad scored on the measure of mother-child interaction, that child's age 3 daily living raw score was predicted to increase by 0.64 units. This indicated support for the hypothesized relationship between one indicator of the early childhood home and family environment, mother-child interaction, and children's adaptive functioning.

Regarding growth in daily living raw scores, the age and squared age term were both significant predictors of daily living raw scores (for age, $\beta=0.98, p<0.01$; for the squared age term, $\beta=-0.002, p<0.01)$. Together, these coefficients indicated that children exhibited increases in their daily living skills over time, and that change became more gradual as children's ages increased. Neither negative life events nor quality of mother-child interaction were significant predictors of growth in daily living raw scores. Diagnosis of motor impairment and diagnosis of developmental delay of unknown etiology were significant predictors of the linear and non-linear slopes. The magnitude and direction of these coefficients were identical in Models 2 and 3, and therefore the 
interpretation remains the same. Figures 15-20 show trajectories for children according to numbers of negative life events (Figures 18-20) and quality of mother-child interaction (Figures 15-17), assuming placement in inclusive settings and mean levels of classroom externalizing problems.

Tables 17-19 contain predicted values of daily living raw scores for children in each of the three disability categories (Down syndrome, motor impairment, and developmental delay of unknown etiology). The predicted values reflect children with high and low levels of early childhood mother-child interaction and high and low numbers of negative life events experienced during early childhood. The "high" and "low" values correspond to values equal to one standard deviation above and below the means for the sample. From Tables 17-19, at each time point, children with high levels of mother-child interaction have daily living raw scores that are approximately 7 units higher than children with low levels of mother-child interaction. From the corresponding age equivalent scores, this indicates a level of functioning that corresponds with approximately 3-4 months of growth that would be expected of typically developing children. Also from Tables 17-19, children who experienced a high number of negative life events have daily living raw scores that are approximately 9 units higher than children who experienced a low number of negative life events. The age equivalent scores indicate that discrepancy corresponds with approximately 5-7 months of growth that would be expected of typically developing children.

The equation below represents Model 3, and includes all significant covariates and predictors. 


$$
\begin{aligned}
& Y_{t i}=24.30+(10.26)\left(D D_{i}\right)-(8.65)\left(C L A S S_{t i}\right)-(3.56)\left(E X T_{t i}\right)+(0.9)\left(N L E_{i}-\overline{N L E} \bullet\right)+ \\
& +(0.64)\left(M C I_{i}-\overline{M C I} \bullet\right)+(0.98)\left(A G E_{t i}\right)-(0.002)\left(A G E S Q_{t i}\right)+(0.47)\left(A G E_{t i} * D D_{i}\right) \\
& +(0.32)\left(A G E_{t i} * M I_{i}\right)-(0.003)\left[A G E S Q_{t i} * D D_{i}\right]-(0.003)\left[A G E S Q_{t i} * M I_{i}\right] \\
& +r_{0 i}+r_{1 i}\left(A G E_{t i}\right)+e_{t i}
\end{aligned}
$$

, where DD represents diagnosis of developmental delay of unknown etiology, MI represents diagnosis of motor impairment, CLASS represents classroom placement type (substantially separate versus inclusive), EXT represents levels of classroom externalizing behaviors, NLE represents the number of negative life events experienced by the family during early childhood, MCI represents the quality of mother-child interaction during early childhood, AGE represents the age of the child, and AGESQ represents the squared age term.

The covariance parameters indicated that there was still significant unexplained variability in initial status $\left(\sigma^{2}{ }_{0}=220.15, p<0.01\right)$ and slope $\left(\sigma^{2}{ }_{1}=0.05, p<0.01\right)$. The addition of predictors representing the early childhood home and family accounted for $10.68 \%$ of the variability in initial status; it did not account for variability in the slope, since the indicators were not significant predictors of the slope. The -2 Log Likelihood and the AIC decreased slightly from Model 2 to Model 3, indicating improvement in fit (2 Log Likelihood $=7961.84$ in Model 2 and 7950.60 in Model 3; AIC $=7989.84$ in Model 2, and 8058.90 in Model 3). However, the BIC increased, indicating that the addition of predictors resulted in worse model fit (8056.60 in Model 2, 8058.90 in Model 3). Model 3 was re-run without the insignificant predictor (diagnosis of motor impairment as a predictor of initial status). This resulted in a smaller BIC (8052.40). However, the final model retained this predictor because it was part of significant interaction effects - the interactions between motor impairment and the linear and 
squared age terms (Chandler \& Scott, 2011). Also, the final model retained the significant predictors representing the early childhood home and family environment, since the AIC and the -2 Log Likelihood suggested that these predictors improve model fit. This is in line with recommendations from Stock and Watson (2007).

\section{Multilevel models predicting total scores of adaptive functioning.}

Unconditional model. Results from the unconditional model are displayed in Table 15. The estimate representing the intercept was equal to 69.45 and was statistically significantly different than zero $(p<0.01)$. The value of this estimate $(69.45)$ represents the average total score of adaptive functioning for the sample at age 3 .

The coefficient representing age was positive and statistically significantly different than zero $(\beta=2.40, p<0.01)$, indicating support for hypothesis 1 , that adaptive functioning scores increase over time. The squared age term was negative and statistically significantly different than zero $(\beta=-0.007, p<0.01)$, indicating a slight decline in rate of growth over time. Figure 4 displays the growth trajectory for total scores of adaptive functioning for the sample as a whole. This figure represents a pattern of overall growth over time, with only a very small decline in rate of growth.

Finally, the significance of the covariance parameters indicated support for hypothesis 2 , that there is variability between people with regard to initial status and rate of change in total scores of adaptive functioning. The value of $\sigma_{0}^{2}$ represents variability in initial status; it was equal to 1517.91 and was statistically significantly different than zero $(p<0.01)$. The value of $\sigma^{2}{ }_{1}$ represents variability in rate of change. It was equal to 0.22 and was also statistically significantly different than zero $(p<0.01)$. 
Model with covariates. Model 2 in Table 15 displays results from the multilevel equations predicting total adaptive functioning scores with covariates representing demographic information from children and families, as well as classroom characteristics and dynamics. The value of the intercept was equal to 69.09 and was statistically significantly different than zero $(p<0.01)$. Taking into account the other variables in the model, this indicates that for children with Down syndrome who were in inclusive settings and who displayed mean levels of externalizing behaviors, the average total adaptive functioning score at age 3 was equal to 69.09 .

The coefficient associated with age was positive and significant, indicating that children displayed increases in total adaptive functioning raw scores over time $(\beta=1.76$, $p<0.01)$. The value associated with the squared age term was not significant $(p>0.05)$. However, as described below, there were significant interactions between covariates and the squared age term.

Diagnosis of developmental delay of unknown etiology was a positive and significant predictor of the intercept $(\beta=28.65, p<0.01)$. This indicates that children with developmental delay of unknown etiology, compared to children with Down syndrome, scored on average 28.65 points higher on the total adaptive functioning measurement at age 3 . The variable representing developmental delay of unknown etiology was also a positive and significant predictor of the linear age term $(\beta=0.86, p<$ 0.01). This indicates that children with developmental delay of unknown etiology displayed more rapid growth in total adaptive functioning than children with Down syndrome. Also, the interaction between developmental delay of unknown etiology and the squared age term was negative and significant $(\beta=-0.007, p<0.01)$. Children with 
developmental delay of unknown etiology, compared to children with Down syndrome, displayed a greater decrease in their rate of growth over time. This decrease is minor; at each time point, children with developmental delay of unknown etiology had higher average total adaptive functioning scores than children with Down syndrome.

Children with motor impairment did not differ from children with Down syndrome in their total adaptive functioning scores at age $3(p>0.05)$. However, diagnosis of motor impairment was a positive and significant predictor of the slope $(\beta=$ $0.72, p<0.01$ ), indicating that children with motor impairment displayed more rapid growth in total adaptive functioning scores than children with Down syndrome. The interaction between diagnosis of motor impairment and the squared age term was negative and significant $(\beta=-0.006, p<0.01)$. Children with motor impairment, compared to children with Down syndrome, displayed a larger decline in their rate of growth over time. That is, their growth slowed more than the growth of children with Down syndrome. This decline was slight, and average scores of children with Down syndrome did not exceed average scores of children with motor impairment at any time point.

Both of the variables representing classroom characteristics and dynamics were significant predictors of initial status of total adaptive functioning. At age 3 , children in substantially separate settings scored on average 18.07 points lower on the adaptive functioning measurement than children in inclusive settings. The coefficient associated with classroom externalizing behaviors was also negative and significant $(\beta=-8.92, p<$ 0.05 ), indicating that children with higher levels of externalizing behavior problems had 
lower total adaptive functioning scores. Neither of these variables was a significant predictor of adaptive functioning rate of change.

The covariance parameters suggested that there was still significant unexplained variability in initial status $\left(\sigma_{0}^{2}=1602.73, p<0.01\right)$ and slope $\left(\sigma^{2}{ }_{1}=0.26, p<0.05\right)$. Model 3 included predictors representing the early childhood home and family environment and attempted to explain this variability.

Finally, the fit statistics from Models 1 and 2 suggested that Model 2 represented a better fit to the data than Model 1. From Model 1 to Model 2, the AIC decreased from 9544.42 to 9324.15 . Similarly, the BIC decreased from 9577.96 to 9390.91 , and the -2 Log Likelihood decreased from 9539.42 to 9296.15.

Model with predictors. Model 3 in Table 15 shows results from the final model, including both covariates and, in order to test hypothesis 3 , predictors representing the early childhood home and family environment. The value of the intercept was equal to 69.34. Taking into account each of the other variables in the model, this value represents the average total adaptive functioning score at age 3 for children with Down syndrome, in inclusive placements, with mean levels of classroom externalizing behaviors, and mean levels of mother-child interaction quality. The coefficient associated with the linear age term was positive and significant $(\beta=1.76, p<0.01)$, indicating that total adaptive functioning scores for children with Down syndrome were estimated to increase by 1.76 units per year. The coefficient associated with the squared age term was not significant $(p>0.05)$, but the squared age term was included in the model because it was part of significant interaction effects (Chandler \& Scott, 2011). 
The following covariates were significant predictors of the intercept: diagnosis of developmental delay of unknown etiology $(\beta=27.23, p<0.01)$, classroom placement type $(\beta=-17.72, p<0.01)$, and levels of classroom externalizing behaviors $(\beta=-8.71, p$ $<0.01)$. The positive coefficient associated with developmental delay of unknown etiology indicates that on average, children with this diagnosis had age 3 total adaptive functioning scores that were 27.73 units higher than the average score of children with Down syndrome. Children in substantially separate settings had age 3 adaptive functioning scores that were on average 17.72 units lower than the scores of children in inclusive settings; for each unit higher that a child scored on the measure of externalizing behavior problems, that child's age 3 total adaptive functioning score was predicted to decrease by 8.71 units.

With regard to hypothesis 3 , significant early childhood home and family predictors of the intercept included negative life events $(\beta=2.25, p<0.01)$ and motherchild interaction quality $(\beta=1.58, p<0.01)$. For each negative life event that a child experienced above the mean number of negative life events, that child's total adaptive functioning score at age 3 was predicted to increase by 2.25 units. Similar to the relationships between negative life events and each of the domains of adaptive functioning, the direction of this relationship is opposite of the hypothesized direction. In support of hypothesis 3 , for each unit increase in the measure of mother-child interaction quality, children's age 3 total adaptive functioning scores were predicted to increase by 1.58 units. The quality of the home environment and child evocative effects were not significant predictors. 
None of the early childhood home and family environment indicators were significant predictors of growth in adaptive functioning total scores. Predictors of the linear slope included diagnosis of motor impairment and diagnosis of developmental delay of unknown etiology ( $\beta=0.72$ and 0.87 respectively, $p<0.01$ for each). The positive values of these coefficients indicate that children in each of these disability categories demonstrated more rapid growth than children with Down syndrome. There were also significant interactions between the squared age term and each of the following: diagnosis of developmental delay of unknown etiology and diagnosis of motor impairment ( $\beta=-0.007$ for developmental delay of unknown etiology, $\beta=-0.006$ for motor impairment, $p<0.01$ for each). The fact that these coefficients were negative indicate that compared to children with Down syndrome, children in each of these disability categories exhibited a more pronounced decline in their rates of growth. This decline, however, was very slight. Figures 21-26 show trajectories for children, with high and low levels of mother-child interaction quality (Figures 21-23) and high and low numbers of negative life events (Figures 24-26).

Tables 17-19 display predicted adaptive functioning total raw scores for children with high and low levels of early childhood mother-child interaction quality, and for children who experienced high and low numbers of negative life events during early childhood. "High" and "low" values correspond with values equal to one standard deviation above and below the means for the sample; Tables 17-19 display predicted values for children with Down syndrome, motor impairment, and developmental delay of unknown etiology, respectively. From these tables, at each time point, children with high levels of mother-child interaction have total adaptive functioning raw scores that are 
approximately 19 units higher than children with low quality mother-child interaction. Additionally, children who experienced a high number of negative life events during early childhood have (at each time point) total adaptive functioning raw scores that are approximately 22 units higher than children who experienced a low number of negative life events. Note that age equivalent scores are not available for total scores of adaptive functioning.

The following equation represents the final model:

$$
\begin{aligned}
& Y_{t i}=69.34+(27.23)\left(D D_{i}\right)-(17.72)\left(C_{L A S S}\right)-(8.71)\left(E X T_{t i}\right)+(2.25)\left(N L E_{i}-\overline{N L E} \bullet\right)+ \\
& +(1.58)\left(M C I_{i}-\overline{M C I} \cdot\right)+(1.76)\left(A G E_{t i}\right)+(0.87)\left(A G E_{t i} * D D_{i}\right) \\
& +(0.72)\left(A G E_{t i} * M I_{i}\right)-(0.007)\left[A G E S Q_{t i} * D D_{i}\right]-(0.006)\left[A G E S Q_{t i} * M I_{i}\right] \\
& +r_{0 i}+r_{1 i}\left(A G E_{t i}\right)+e_{t i}
\end{aligned}
$$

, where DD represents diagnosis of developmental delay of unknown etiology, MI represents diagnosis of motor impairment, CLASS represents classroom placement type (substantially separate versus inclusive), EXT represents levels of classroom externalizing behaviors, NLE represents the number of negative life events experienced by the family during early childhood, MCI represents quality of mother-child interaction during early childhood, AGE represents the age of the child, and AGESQ represents the squared age term.

The covariance parameters indicated that there was still significant variability in both intercept $\left(\sigma_{0}^{2}=1440.06, p<0.01\right)$ and slope $\left(\sigma^{2}{ }_{1}=0.26, p<0.01\right)$. The early childhood home and family environment predictors accounted for $10.15 \%$ of the variability in initial status; they did not account for variability in slope, since they are not significant predictors of the slope. The AIC and the -2 Log Likelihood decreased from Model 2 to Model 3, indicating improvement in model fit (-2 Log Likelihood $=9296.15$ 
in Model 2, 9283.45 in Model 3; AIC = 9324.15 in Model 2, 9315.45 in Model 3).

However, the BIC increased slightly (9390.91 in Model 2, 9391.74 in Model 3). The AIC was selected as the fit statistic and Model 3 was retained as the final model, in order to include all significant predictors (Stock and Watson, 2007) and all predictors included in interaction terms (Chandler \& Scott, 2011).

Negative life events: follow-up analyses. Results from multilevel models indicated that negative life events were positively and significantly associated with each of the adaptive functioning outcomes, including socialization, communication, daily living skills, and total scores of adaptive functioning. Children whose families experienced more negative life events during early childhood had higher scores on each of the adaptive functioning outcomes. Since the direction of this relationship is opposite that of the hypothesized direction, multiple additional analyses were completed in order to illuminate relevant processes.

From Table 6 , the mean number of negative life events experienced by families was equal to $6.99(\mathrm{SD}=4.89)$. There was a large range, with some families experiencing no events $(\mathrm{n}=4)$ and one family experiencing as many as 26 . Note that each multilevel analysis was completed excluding this one outlier that had experienced 26 negative life events; the results were unchanged.

First, analyses were conducted to explore the possibilities that families including children with Down syndrome experienced fewer negative life events than other families, and that children with Down syndrome had lower levels of adaptive functioning than other children. Researchers have identified demographic differences between families of children with Down syndrome and families including children with 
other developmental disabilities, including older parental age, higher income levels, and higher levels of parental educational attainment (Corrice \& Glidden, 2009). Research has also indicated that children with Down syndrome and their mothers have more positive interactions than mother-child dyads that include children with other disabilities (Mitchell, Hauser-Cram, \& Crossman, 2014). All of these factors, including higher socioeconomic and relational resources, might buffer families from the experience of negative life events. At the same time, as Table 9 indicates, children with Down syndrome had lower scores than other children on many of the adaptive functioning outcomes.

In order to account for this possibility that the positive relationship between negative life events and adaptive functioning reflects unique characteristics of children with Down syndrome and their families, multilevel analyses were re-run with the inclusion of a variable representing diagnosis of Down syndrome $(1=$ diagnosis of Down syndrome, $0=$ other diagnostic category). However, the relationship between negative life events and adaptive functioning remained positive and significant.

Additional analyses were conducted in order to further explore the types of negative events that families experienced, and how these individual events might relate to family and child functioning. Table 16 displays the number of families that experienced each kind of negative life event. The most commonly experienced events were financial: experiencing a decrease in income and going into debt. It is possible that these events weren't large stressors for the families in the current sample, since most families were reasonably well-off financially (see Table 7). The most common negative life event, a substantial decrease in income, might have occurred voluntarily for families; parents 
(especially mothers) might have decided to reduce work hours in order to spend more time with their children, many of whom had special care needs. If this is the case, the experience of this life event in particular might be more likely to occur in families of relatively high socioeconomic status. The data, however, do not support these hypotheses. Experiencing a decrease in income was negatively associated with family income level $(r=-0.26, p<0.01)$, indicating these decreases do not reflect voluntary decisions made by families who were relatively wealthy. Also, the number of negative life events experienced by families was negatively associated with both mother-child interaction quality $(r=-0.14, p<0.01)$ and the quality of the home environment $(r=$ $0.19, p<0.01)$. In general, negative life events did not appear to be associated with positive family functioning.

The data did not provide empirical support for several potential explanations for the positive relationship between negative life events and adaptive functioning. However, the discussion chapter includes additional potential explanations, which future research can investigate further. 


\section{Chapter 5: Discussion}

This dissertation is the first study to examine trajectories of school-based adaptive functioning for children with DD. As such, it provides much needed information about the timing and extent of growth in this domain. In addition, it includes valuable information about individual differences that pertain to levels of adaptive functioning at individual time points and rates of growth. This dissertation builds on literature that indicates the salient and enduring effects of the early childhood home environment for typically developing children (e.g., Bowlby, 1973; Bronfenbreener \& Morris, 2006; Roisman \& Fraley, 2013; Sroufe et al., 1990) and children with DD (Hauser-Cram et al., 2001). Finally, this dissertation is one of few studies of children with DD that has explored whether the influence of these effects extends into the school setting (Baker et al., 2007). It is the only study that has explored the influence of the early childhood home and family environment on trajectories of school-based adaptive functioning for children with DD.

This discussion chapter highlights primary findings and their implications. It also indicates where the findings of this dissertation point to the need for future research. Specifically, the discussion includes the following: (1) an examination of the trajectories of growth for the whole sample and for each diagnostic category, (2) a summary of main findings related to early childhood home and family environment predictors of schoolbased adaptive functioning, (3) possible explanations regarding the observed relationships between early childhood home and family indicators and school-based adaptive functioning, and (4) an analysis of the practical significance of findings related to the early childhood home and family environment, and related implications. The 
discussion concludes with a summary of the limitations and contributions of this dissertation, including identified areas for future research.

\section{Trajectories of Growth}

Results from this dissertation provide clear support for hypothesis 1 , that there are increases in raw scores on classroom adaptive functioning in each domain, and in total scores of adaptive functioning, over time. In each of the unconditional models (representing socialization, communication, daily living skills, and total scores), the coefficient associated with the linear age term was positive and significant $(\beta=0.40$ for socialization, 0.71 for communication, 1.29 for daily living skills, and 2.40 for total adaptive functioning scores). Since the unit of time in these analyses is the year, each year children's adaptive functioning raw scores are predicted to increase by the value of these coefficients.

This general pattern of growth (i.e., the finding that growth occurs over time) is consistent with research that has examined trajectories of adaptive functioning for children with Down syndrome, ASD, motor impairment, and developmental delay of unknown etiology (Baghdadli et al., 2012; Hauser-Cram et al., 2001). However, this previous research examined trajectories of adaptive functioning as displayed in the home; very little was known about trajectories of school-based adaptive functioning. The finding that adaptive functioning at school generally improves with age is important in light of the following: "an important assumption about adaptive functioning is that with appropriate supports and interventions for a person's adaptive skills deficits, life functioning will generally improve" (Harrison \& Boney, 2002, p. 1168). 
It is also important to consider the magnitude of growth. The use of raw scores prohibits cross-domain comparison (Sparrow et al., 1985). Each domain has a different number of items, and total possible scores vary accordingly. The socialization, communication, and daily living skills domains have 53,63, and 99 items respectively. A raw score of 40 in the socialization domain therefore suggests a different level of functioning than the same raw score in the daily living skills domain. From the unconditional models, the coefficients representing age related growth are equal to 0.40 for socialization, 0.71 for communication, 1.29 for daily living skills, and 2.40 for total scores. For each year, children's raw scores are predicted to increase by 0.40 units in the socialization domain, 0.71 units in the communication domain, 1.29 units in the daily living skills domain, and 2.40 units in total adaptive functioning scores. However, in light of the inability to draw cross-domain comparisons, it is inappropriate to conclude that children exhibit more growth in the daily living skills domain than in the other two domains.

In order to evaluate the magnitude of growth reflected by these coefficients, it is important to consider the unit of analysis: raw scores of adaptive functioning. Raw scores are calculated by summing the responses to each item in a domain (the raw total score reflects the sum of the three domain raw scores). For each item, teachers indicated the extent to which the child exhibited the specified behavior $(2=$ yes, usually, $1=$ sometimes or partially, $0=$ no, never). Each domain contains items that vary in complexity and level of abstraction. An example of a relatively complex item is, "controls anger or hurt feelings when denied own way" (from the socialization domain); while a more straightforward item is "gets drink of water from tap unassisted" (daily 
living domain). It is likely that once a child is able to get water, he or she will do so with great consistency. It is also likely that displays of emotional regulation will be somewhat sporadic, and will depend on factors including the intensity of emotion (Aldao \& NolenHoeksema, 2012). Therefore, it might be relatively "easy" for a child to jump from a 0 to a 2 on some items, while change might be more gradual for other items.

In light of these considerations, the observed coefficients potentially represent meaningful growth in adaptive functioning. The transition from sporadic to consistent completion of a single task (represented by a score increase of a single unit) can have important implications for an individual's levels of independence and functioning. Examples of relevant tasks from the socialization, communication, and daily living skills domains include respectively "identifies people by characteristics other than name, when asked", "articulates clearly, without sound substitutions", and "dresses self completely, except for tying shoelaces". Typically developing children achieve these tasks at approximately three years of age (Sparrow et al., 1984).

In addition to support for the hypothesis that raw scores of adaptive functioning increase over time, the unconditional models representing communication, daily living skills, and total scores also include a negative and significant coefficient representing the squared age term. This indicates that in each of these domains, rate of change becomes more gradual as children age. This finding will be discussed as it pertains to the sample as a whole, and caveats regarding the small magnitude of the coefficients will be presented. Then, since subsequent analyses indicated significant interactions between disability diagnostic category and the squared age term, the discussion of disabilityspecific trajectories will also include interpretation. 
Some researchers have suggested that adaptive functioning of some children with DD reaches a ceiling level; that is, growth may cease once children reach a certain age or a certain level of functioning (van Duijn, Dijkxhoorn, Scholte, \& van Berckelaer-Onnes, 2010). Despite the negative and significant squared age term, the sample in the current study does not demonstrate a ceiling level of adaptive functioning in any domain. Figures 2-4 display the trajectories of growth for the outcomes that included a negative and significant squared age term as a predictor: communication, daily living skills, and total scores. In these figures, it is apparent that growth does not level-off in the time frame included in the current study. The decline is minor relative to the rate of change and does not suggest an absence of growth at any time point.

While Figures 2-4 do not indicate a specific age at which a steep decline in growth occurs, the finding that a decline does occur is consistent with a study of children with ASD that examined trajectories of adaptive functioning and observed a decline in rate of growth in middle childhood (Baghdadli et al., 2012). Baghdadli et al. (2012) obtained parental report of children's socialization, communication, and daily living abilities at ages 5,8 , and 15 . While the trajectories of some children were characterized by low levels of growth throughout this entire time period, the trajectories of a second group of children displayed especially pronounced growth between ages 5 and 8 (Baghdadli et al., 2012). These authors provide multiple possible explanations for the subsequent decline in rate of change (after age 8), including the following: children's stress levels increase as a result of increasing environmental demands and their functioning is impacted accordingly; access to services may decrease thus limiting opportunities for involvement in developmentally rich activities; instruments commonly 
used to assess adaptive functioning might not be sensitive to changes that occur in adolescence (Baghdadli et al., 2012). The findings from this dissertation cannot support nor refute any of these possibilities. However, the results of this study do suggest that future research should identify explanations for the observed decrease in growth rates, so that interventions can be designed accordingly. Given the context dependency of adaptive functioning, children's homes, schools, and communities should facilitate constant growth (Harrison \& Boney, 2002).

It is also worthwhile to consider the finding that socialization raw scores, unlike scores in the other domains, were not predicted by a squared age term. The rate of change remained consistent throughout the time frame examined; rate of change neither increased nor decreased. The lack of a decline in rate of change in this area might partially reflect sample characteristics, including factors that vary within and across disability categories. While Baghdadli et al. (2012) found that rates of growth in socialization declined after age 8 for children with ASD, Dykens et al. (2006) observed a different pattern in a sample of children with Down syndrome. These authors used a cross-sectional design to examine trajectories of growth in children with Down syndrome ages 1 to 11.5. They compared young children ages 1 to 6 to older children (from above age 6 to age 11) and found that the older group displayed pronounced variability in rate of change (Dykens et al., 2006). After age 6, growth in socialization reached a ceiling level for some of the children with Down syndrome. While children with ASD are not included in the sample for this dissertation, the finding that these children are especially likely to display a decline in rate of socialization growth indicates that this decline might be partially dependent on disability diagnosis (Baghdadli et al., 2012). It likely also 
depends on individual-level factors that pertain to children within diagnostic categories (Dykens et al., 2006).

The trajectories provide consistent support for hypothesis 2 , that there is variability between people in initial levels of school-based adaptive functioning and in rates of change. This is consistent with the body of work that has examined adaptive functioning as assessed via parent-report (Anderson et al., 2009; Baghdadli et al., 2012; Dieterich et al., 2004; Fenning \& Baker, 2012; Hauser-Cram et al., 1999; Hauser-Cram et al., 2001; Warfield, 1994). In the current study, for each domain and for total scores of adaptive functioning, unconditional models indicated significant variability in both initial status and rates of change. The following discussion includes explanations for, and implications of, this observed variability. The explanations for observed variability include characteristics of specific disabilities, as well as indicators of the early childhood home and family environment.

Disability-specific trajectories. The figures that display the unconditional models (Figures 1-4) and the figures that display the influence of the early childhood home and family environment on trajectories of school-based adaptive functioning (Figures 5-26) show the trajectories for children in individual categories. As these figures indicate, children who were diagnosed during early childhood as having developmental delay of unknown etiology have higher raw scores at age 3 than do children with Down syndrome on each of the four outcomes (socialization, communication, daily living skills, and total scores). Also compared to children with Down syndrome, children with developmental delay of unknown etiology have more rapid growth in their daily living skills, communication skills, and total scores. For 
communication, daily living skills, and total scores, the rate of growth for children with developmental delay lessens over time more than it does for children with Down syndrome. In general, children with developmental delay of unknown etiology have a higher initial status and overall rate of growth than children with Down syndrome, but their rate of growth declines over time. At each time point and for each outcome, children with developmental delays of unknown etiology have higher raw scores than children with Down syndrome.

A similar pattern describes the differences between children with motor impairment and children with Down syndrome. In the socialization domain, there are no differences between children with motor impairment and children with Down syndrome. In the daily living skills and communication domains, children with motor impairment have a higher initial status and more rapid rate of change than children with Down syndrome. Also for both of these outcomes, children with motor impairment demonstrate a decrease in rate of change over time; their growth slows more than that of children with Down syndrome. On total scores of adaptive functioning, there is no difference in initial status between children with motor impairment and children with Down syndrome, but children with motor impairment display a more rapid rate of change and also a more pronounced decline in this rate of change over time.

In general, children with Down syndrome have lower adaptive functioning scores at each time point than children in the other two diagnostic categories. Children with Down syndrome have initially lower levels of adaptive functioning (at age 3) and slower rates of growth. While their rates of growth decline less (or stay more stable) than the rates of growth of other children, this difference does not eliminate the gap between the 
groups of children: adaptive functioning raw scores of children with Down syndrome remain lower than their peers with other DD. The exception is that there is no difference between children with Down syndrome and children with motor impairment in the socialization domain; this possibly reflects the fact that for children with Down syndrome, socialization is a relative strength (Dykens et al., 2006).

The findings indicating relatively low levels of adaptive functioning in children with Down syndrome are consistent with research that has found relationships between adaptive functioning and both intelligence and levels of behavior problems (de Bildt et al., 2005; Gligorovic \& Durovic, 2014). In the current sample, compared to children in the other two diagnostic categories, children with Down syndrome had statistically significantly lower IQ scores and higher levels of classroom externalizing behaviors.

The finding that children with Down syndrome maintain a relatively stable rate of growth (i.e., their rate of growth declines less over time than rates of growth for children in other diagnostic categories) is fairly novel. One speculation relates to the fact that while most children with DD eventually exhibit declines in growth rates, this may occur relatively late for children with Down syndrome. For example, research has suggested that children with Down syndrome reach ceiling levels in adaptive functioning in early adolescence (Van Duijn et al., 2010). In contrast, children in other diagnostic categories have been found to exhibit declines in growth rates around age 8 (Baghdadli et al., 2012). Given the ages assessed in the current study (with the last adaptive measurement occurring at age 15), the findings from Van Duijn et al. (2010) would suggest that a decline would be evident in children with Down syndrome. However, since children with other disabilities exhibit earlier declines, the time frame of the current study might 
make these declines comparatively less evident for children with Down syndrome than for children with other disabilities. A second speculation relates to the possibility that families including a child with Down syndrome might have been able to access resources that would facilitate consistent development. This is consistent with research that suggests that these families, compared to families including children with other disabilities, have advantages including higher levels of socioeconomic status (Corrice \& Glidden, 2009).

In light of these differences between diagnostic categories, a caveat is warranted. The trajectories for each individual diagnostic category still conform to the general patterns described above: (1) children in each category gain skills over time (their raw scores increase), and (2) while growth may decline over time (especially for children with motor impairment and developmental delay of unknown etiology), the decline is small. Since there is not an absence of growth at any time point, the decline does not indicate a ceiling effect.

The examination of trajectories provides the opportunity to identify moments in development in which intervention might be particularly essential (Anderson et al., 2009). Given the general pattern of increase over time for children in each diagnostic category, the findings from this dissertation do not indicate a time point at which intervention may be especially crucial. However, given the context dependency of adaptive functioning (Harrison \& Boney, 2002), teachers and other school-based service providers should always ensure that the school context facilitates optimal functioning for all.

\section{Early Childhood Home and Family Environment Predictors}


Results of this study indicate support for hypothesis 3 , that indicators of the early childhood home and family environment account for variability in adaptive functioning initial status and rates of change. The quality of mother-child interaction predicted growth in socialization raw scores, with higher levels of mother-child interaction relating to more rapid growth. The quality of mother-child interaction also predicted communication, daily living skills, and total age 3 scores (initial status).

In addition, the number of negative life events experienced by the family during early childhood predicted age 3 scores on each of the four outcomes (socialization, communication, daily living skills, and total raw scores). However, for each of these outcomes, the direction of the relationship was opposite of the hypothesized relationship: higher numbers of negative life events were associated with higher adaptive functioning raw scores. The role of each hypothesized predictor is discussed in the next section, and this discussion includes potential explanations for the salience of mother-child interaction, relative to the other predictors. This discussion also includes speculation about the observed and unexpected positive relationship between negative life events and school-based adaptive functioning.

The salience of mother-child interaction. Results from this study point to the quality of mother-child interaction as an especially salient predictor of school-based adaptive functioning. Multiple theoretical perspectives, including attachment theory (Bowlby, 1969) and socialization theory (Bugenthal \& Grusec, 2006) highlight children’s early relationships with their caregivers as important developmental contexts. Through these relationships, children develop perceptions of self and others. They carry these perceptions through place and time, and their subsequent experiences and interactions are 
impacted accordingly. Also, high quality relationships enable infants to learn how to cope with new and potentially stressful circumstances, since children trust their caregivers and are able to effectively utilize them as sources of comfort (Landry et al., 2006). This coping facilitates the development of self-regulation (Landry et al., 2006). Both self-regulation and trust in caregivers promote exploration, communication, and cooperation (Landry et al., 2006).

This theoretical premise provides a framework for understanding why high quality mother-child interaction gives children skills and dispositions that are particularly likely to manifest in external contexts (in this case, the school). Children with positive perceptions of themselves may be relatively likely to persist in challenging situations, regardless of whether these challenges occur at home or at school, since they likely have confidence in their ability to ultimately succeed. Research with typically developing children supports this link between self-perception and constructs related to persistence, including mastery motivation (Gilman \& Anderman, 2006). The following examples indicate how these processes might impact classroom-based adaptive functioning. A child with positive beliefs about his or her capabilities may continue with communication attempts despite being misunderstood; may put away his or her own belongings instead of accepting someone's offer of assistance; and may decide to participate in extracurricular activities despite challenges inherent in these activities.

Also, children with positive perceptions of others are likely to view these others as potential sources of help, support, and friendship. Research with typically developing children indicates that children with higher quality relationships with their mothers during early childhood have higher quality relationships with peers (West, Mathews, \& 
Kerns, 2013) and teachers (Verschueren, Doumen, \& Buyse, 2012) once they reach school age. For children with DD, these enhanced relationships can increase children's willingness or ability to utilize their teachers and peers as sources of support. Higher quality relationships with teachers and peers, while not assessed in this dissertation, might also enhance the willingness and / or ability of these individuals to provide appropriate supports to children. This availability of support, in turn, can potentially enhance adaptive functioning.

Very few studies including children with DD have investigated the transfer of influences from the early childhood home environment to the school setting, and none have explored links between early childhood mother-child interaction and subsequent teacher-student relationships. However, Baker et al. (2007) investigated links between maternal scaffolding, a component of mother-child interaction, and school-based peer social skills, in a sample of 6-year-old children with developmental delays. Higher levels of maternal scaffolding were found to predict higher levels of social skills (Baker et al., 2007). Generally, this study provides support for the hypothesis that for children with DD, mother-child interaction quality promotes school-based social skills. Future studies should test the hypotheses that these social skills enable children to interact effectively with teachers as well as peers, and that all of these enhanced interactions contribute to levels of adaptive functioning.

It is also worth considering the predictive nature of mother-child interaction quality in light of the fact that two other indicators of the early childhood home and family environment, child evocative effects and the quality of the home environment, were not predictive of classroom-based adaptive functioning. Contextual theories of 
child development posit that outcomes observed in one setting, including levels of adaptive functioning as observed in the school, are partially susceptible to processes that occurred in other times and places, including the early childhood home and family environment (Bronfenbrenner \& Morris, 2006). From this perspective, mother-child interaction, the quality of the home environment, and child evocative effects as displayed in the home, all constitute experiences whose influences should transcend to the school. However, mother-child interaction may be unique because of additional theoretical emphases on relationships with caregivers that transpire during the early childhood period in particular, and the capacity of these relationships to have enduring effects (Bowlby, 1969; Bugenthal \& Grusec, 2006). As described above, through these relationships children develop views of self and other that they carry forward through place and time.

Differential influence of mother-child interaction across domains. The role of mother-child interaction as a predictor of growth in socialization warrants attention, since it predicted initial status (and not growth) in each of the other three outcomes. The lack of influence on initial status suggests that the effects of mother-child interaction on socialization might appear after age 3. This is consistent with the idea that the impact of high quality home and family environments on adaptive functioning might emerge after the sensorimotor period, once children have acquired baseline skill levels (Hauser-Cram et al., 2001). At age 3, children in the current sample have scores in the socialization domain that are comparable to their scores in other domains (mean age equivalents are equal to 1 year 7 months for socialization; 1 year 5 months for communication; and 1 year 8 months for daily living skills). However, their functioning related to socialization 
at this age might be particularly dependent on factors including the child's cognitive ability. For example, one task in the socialization domain that typically developing children achieve between one and two years of age is engagement "in elaborate makebelieve activities, alone or with others". Therefore, other processes including general cognitive development might be necessary before the influence of mother-child interaction can be observed.

Once higher levels of socialization skills emerge as a result of high quality mother-child interaction, these socialization skills might have especially salient evocative effects. A child with high levels of socialization skills might be especially like to evoke positive responses from caregivers and peers; these positive interactions can contribute to subsequent growth (thus explaining the role of mother-child interaction as a predictor of growth) (Lifter, Foster-Sanda, Arzamarski, Briesch, \& McClure, 2011). Research including children with disabilities supports this possibility that higher levels of social skills are associated with higher levels of acceptance (or more positive evocative effects) from others in the school environment (Green, Drysdale, Boelema, Smart, van der Meer, Achmadi, ...\& Lancioni, 2013; Odom, Zercher, Li, Marquart, Sandall, \& Brown, 2006). For example, one study evaluated the impact of a video modeling program designed to enhance the social skills of children who exhibited deficits in this area (Green et al., 2013). Teachers and parents indicated that the improvements in social skills led to increases "in the amount of time engaged in positive social interactions with peers" (Green et al., 2013). This time potentially constitutes a developmental context in which social skills can be further enhanced (Lifter et al., 2011). 
Child evocative effects and the quality of the home environment. Multilevel analyses indicated non-significant relationships between school-based adaptive functioning and both of the following: child evocative effects and the quality of the home environment. Possible explanations for the non-significance of these predictors are discussed in turn.

Regarding child evocative effects, one explanation relates to the possibility that child characteristics have differential impacts across the home and school contexts. The composite representing child evocative effects was comprised of the following: mothers' and fathers' ratings of children's adaptability, demandingness, and mood. This composite was significantly and negatively associated with other indicators of the early childhood home and family environment, including mother-child interaction and the quality of the home environment $(r=-0.11, p<0.01 ; r=-0.09, p<0.05$, respectively). Therefore, more stressful evocative effects possibly exerted a negative impact on children's opportunities within the home. However, the characteristics that parents perceive to be stressful might exert neutral or even adaptive effects in the classroom context. A child who is demanding, for example, might be adept at competing with classmates for attention and help from teachers. This is consistent with the argument that dynamics between teachers and students is drastically different from the dynamics between parents and their children (Parsons, 1959).

Multilevel analyses also indicated that the quality of the home environment is not a significant predictor of school-based adaptive functioning. This contrasts with findings from an earlier study that identified relationships between the quality of the home environment and adaptive functioning as measured by parent report for children with 
Down syndrome (Hauser-Cram et al., 1999). Taken together, this body of work indicates that while the quality of the home environment can potentially influence adaptive functioning for children with $\mathrm{DD}$, this influence does not necessarily transfer to the school setting. It is possible that transfer is dependent on factors such as communication between parents and teachers, so that children can consistently access similar supports across the home and school learning environments. As others have noted, teachers of students with disabilities need information from parents in order to "gear the support for particular children to their individual needs" (Fonteine, Zijlstra, \& Vlaskamp, 2008, p. 477). Other relevant factors may include child characteristics, including motivation to exhibit behaviors that may be required or encouraged at home, but perhaps receive less emphasis at school.

Negative life events. Research with typically developing children (Ostberg \& Hagekull, 2013) and children with DD (Heyman \& Hauser-Cram, 2015) has identified links between the experience of negative life events and developmental outcomes. For typically developing children, these outcomes include behavior problems and social competence (Ostberg \& Hagekull, 2013); for children with DD relationships have been identified between negative life events and executive function (Heyman \& Hauser-Cram, 2015). For both typically developing children and children with DD, the experience of more negative life events is associated with less optimal developmental outcomes, including higher levels of behavior problems, lower levels of social competence, and less efficient executive function (Heyman \& Hauser-Cram, 2015; Ostberg \& Hagekull, 2013).

Potential explanations for these relationships will be considered in the context of the observed positive relationship between negative life events and adaptive functioning. 
One explanation reflects the premise that negative life events are an indicator of toxic stress, and this stress is experienced by the children whose development is observed. The experience of chronic stress results in consequences including cortisol imbalance, and this can have a detrimental impact on the developing brain (Shonkoff et al., 2012). Children can experience stress via at least two distinct pathways. First, the events themselves can be stressful for the children. Second, the events can impact other family members, and strained relationships within the family can be sources of stress for the children, or can impact children's relationships with family members due to family members' heightened levels of stress, depression, and so on. In support of this second pathway, research including children with disabilities has identified relationships between negative life events and maternal stress (Warfield et al., 1999), as well as relationships between parental stress and child social competence (Guralnick et al., 2003).

Importantly, the children in the current study were very young during the experience of recorded negative life events; it is unlikely that they were sufficiently aware of events to experience them as direct sources of stress. Even if they had been older and were cognizant of the occurrence of events, children and their parents do not always perceive the same events as being stressful. Research with typically developing children has suggested that there are often discrepancies in the number of events reported by parents versus children, and this suggests the importance of multiple reporters in the measurement of negative life events (Johnston, Steele, Herrera, \& Phipps, 2003). This provides one potential explanation for the lack of a statistically significant negative relationship between negative life events and adaptive functioning. 
However, it is also important to consider the counter-intuitive finding that negative life events were associated with positive outcomes- heightened levels of adaptive functioning. This finding will be considered in light of the second pathway connecting negative life events and child outcomes: negative life events impact family members and family functioning, and these changes within the family impact children's proximal developmental contexts and opportunities. Therefore, one possibility relates to the fact that for some families, negative life events can actually represent adaptive processes that enhance family functioning, and children's development can benefit accordingly.

For example, a decrease in income is generally considered negative and stressful. However, for families with young children it might represent an adaptive process if it accompanies parents' voluntary decisions to leave or reduce employment, in order to spend more time with their children. This might be especially relevant in families that include a child with DD, since these children often have time-consuming healthcare and service coordination needs (Green, 2007). However, as described in the results section, the data did not support this hypothesis: the experience of more negative life events was associated with lower socioeconomic status, and therefore unlikely reflects voluntary decreases in income.

Death of a family member is an additional example of a negative life event that can, in some instances, represent an adaptive process. In the current study, mothers indicated if any family member had died, including individuals from their families of origin. In some cases, it is likely that children's aging grandparents passed away, thus relieving children's parents of the stressors associated with caring for their own aging 
parents. Research has identified relationships between caregiving burden experienced by adult children of aging parents and negative outcomes for these adult children including increases in marital stress and decreases in self-esteem (Ron, 2006). And, research including children with disabilities has identified links between parents' stress levels and children's outcomes, including social competence (Guralnick et al., 2003). Therefore, while grief is integral to the experience of losing a family member, the alleviation of a stressor might also enhance family functioning in the child's immediate family. In these cases, children's opportunities for development would be enhanced accordingly.

A final potential explanation for the positive and unexpected relationship between negative life events and adaptive functioning involves the role of social support. For parents of children with DD, social support has been found to predict reductions in parental stress for both mothers and fathers (Hauser-Cram et al., 2001), as well as enhanced quality of parent-child interaction (Dunst, Trivette, \& Cross, 1986). Other research has indicated that for families of young children, family-centered intervention practices, which include the provision of social support, positively contribute to children's developmental outcomes, including their cognitive and language development (Trivette, Dunst, \& Hamby, 2010). Taken together, this research indicates that social support enhances parental well-being, which contributes to positive family functioning; enhanced levels of family functioning subsequently promote child development.

The influence of social support relates to the experience of negative life events because many of these events are associated with the receipt of social support. For example, when families experience the death of a family member or loss of a job, their social networks (including neighbors, religious organizations, etc.) may assume a 
prominent role in providing support. This support can take several different forms preparing a meal, checking in via a telephone call, or offering to provide childcare. All forms of support can demonstrate the availability of social resources to the family. Many families will choose to access these resources, and may continue to do so after the negative life event loses some of its saliency. Negative life events may therefore be the impetus that connects a family to its larger community. The family and the child can benefit accordingly. Admittedly, this dissertation offers limited support for these processes, since measures of social support were not included. Also, the experience of negative life events was negatively associated with quality of mother-child interaction. However, given the positive relationships between negative life events and adaptive functioning in each domain, future research should explore these possibilities; if these suggested processes operate, it will be important to identify methods of connecting families to much-needed support.

Practical significance and implications. Findings related to the enduring effects of the early childhood home and family environment have significant policy and program implications for children with DD. According to federal law, children with DD from birth to age 3 are eligible to receive early intervention services (EI) (United States Department of Education, 2014). These services are available in every state and seek to enhance children's development through a family-centered service delivery system, with possible services including training and / or the provision of support for parents. Therefore, results from this dissertation lend empirical support to the recommendation that encouraging mothers to effectively interact with their young children should be a priority within the constellation of EI services. This is especially relevant given research 
indicating positive effects of interventions aimed at improving interactions between mothers and their young children with DD (Kim \& Mahoney, 2005; Mahoney \& Perales, 2005). Participation in these interventions has been shown to predict improved quality of mother-child interactions, and also growth in children's developmental outcomes, including language and social capabilities (Kim \& Mahoney, 2005; Mahoney \& Perales, 2005).

It is important to consider the size of observed effects prior to recommending or instituting policy or program change, however (McCartney \& Rosenthal, 2000). At age 15 , children with mother-child interaction scores one standard deviation above the mean had socialization raw scores that were approximately 0.57 units higher than the socialization raw scores of children with mother-child interaction scores equal to one standard deviation below the mean. Difference in communication raw scores between children with high and low mother-child interaction scores were equal to 7.02 units at each time point; the difference in daily living skills raw scores was equal to 7.62 units at each time point; and the difference in total adaptive functioning raw scores was equal to 18.80 at each time point. As previously noted, the use of raw scores precludes crossdomain comparison (Sparrow et al., 1985). Each domain has a different number of items, and total possible scores also differ. The seemingly low effect associated with the influence of mother-child interaction on socialization skills might simply reflect that the socialization domain, in comparison to the other domains, has the fewest number of items.

In light of this caveat, each effect size has the potential to represent meaningful change in adaptive functioning. The difference in socialization raw scores associated 
with high versus low quality mother-child interaction is equal to 0.57 at age 15 . The value of 0.57 represents the difference between children with mother-child interaction scores one standard deviation above the mean, and children with mother-child interaction scores one standard deviation below the mean. On average, children's socialization raw scores increased by 4.97 units between the ages of 3 and 15; the value of 0.57 is therefore equal to $11.5 \%$ of the total magnitude of growth exhibited by children between the ages of 3 and 15. An increase of 1.0 corresponds with occasional or partial demonstration of one novel task, so the effect does not suggest an improvement of that magnitude. However, as described elsewhere, many tasks in the socialization domain are complex (i.e., "follows school rules"). This particular task involves the coordination of multiple developmental competencies, as students must understand school rules, employ behavioral inhibition to comply with rules despite temptation to do otherwise, etc. Also, the skills involved in following school rules might change over time, as expectations increase throughout one's schooling. In light of these complexities, even small increments of progress might represent meaningful levels of enhanced functioning. Future studies should explore relationships between observed growth and meaningful outcomes associated with adaptive functioning, including friendships and participation in social activities.

At each time point, children with mother-quality interaction scores equal to one standard deviation above the sample mean had predicted daily living and communication raw scores that were 7 points higher than children with mother-child interaction scores one standard deviation below the sample mean. In the communication domain, the mean score at age 3 was equal to 24.07 , and the mean score at age 15 was equal to 89.06 . 
Therefore, the seven units associated with high quality mother-child interaction represent approximately $10.8 \%$ of the total average growth exhibited by the sample $(7 /[89.06-$ 24.07]). The average daily living score at age 3 was equal to 23.61 , and at age 15 was equal to 102.78 . In the daily living domain, 7 units represent $8.8 \%$ of the total average growth $(7 /[102.78-23.61])$.

An increase of 7 raw points also corresponds with the consistent display of more than three additional behaviors (since a consistent display of one behavior corresponds with two raw points). Many of the behaviors in these domains have important implications for levels of independence and overall functioning. Examples include "demonstrates understanding that hot things are dangerous", "uses the telephone for all kinds of calls, without assistance", "bathes or showers completely and dries self adequately, without assistance" (daily living skills domain); "listens attentively to instructions when spoken to directly"; "says at least 100 recognizable words"; and "states telephone number when asked" (communication domain). Across all of the domains, children with high levels of mother-child interaction exhibit nine more independent behaviors (raw score difference $=18.80)$ than children with low levels of mother-child interaction.

The observed effects, therefore, suggest the importance of programs aimed at enhancing mother-child interaction quality for mothers and their young children with DD. These programs can be integrated and / or expanded within the existing early intervention service system. Mother-child interaction is an especially relevant target in light of research indicating that mothers confront unique challenges related to their interactions with their children with DD (Barnard \& Kelly, 1990), in comparison to mothers of 
typically developing children. Finally, extant studies support the effectiveness of relevant interventions, with outcomes including enhanced mother-child interaction quality and positive developmental outcomes for children ((Kim \& Mahoney, 2005; Mahoney \& Perales, 2005).

\section{Conclusions}

The concluding section of this chapter describes limitations of this dissertation, and identified directions for future research. It also contains a summary of the contributions of this dissertation.

Limitations and directions for future research. In light of the salience of mother-child interaction, one limitation of this dissertation is the inability to identify specific elements of mother-child interaction that positively contribute to the development of school-based adaptive functioning. The measure of mother-child interaction used in the current study consists of the sum of scores from four subscales: sensitivity to cues, response to distress, social-emotional growth fostering, and cognitive growth fostering (Barnard, 1978). It is possible that each of these characteristics (sensitivity to cues, response to distress, social-emotional growth fostering, and cognitive growth fostering) provide a unique contribution to children's adaptive functioning capabilities. It is also possible that certain characteristics are especially important for adaptive functioning in general or for specific domains of adaptive functioning in particular. Social-emotional growth fostering, for example, might be particularly relevant for growth in the socialization domain, which encompasses skills that include "controls anger or hurt feelings when denied own way." However, cognitive-growth fostering might also contribute to development in the socialization domain; several items in this 
domain relate to utilization of play and leisure time (i.e., "has a hobby"). Mothers who are skilled at providing cognitive support to their children as they complete a novel task (during the observed mother-child interaction task) might also provide appropriate support to their children in their pursuit of challenging and novel leisure time activities (i.e., playing a musical instrument, sewing, etc.).

More generally, a limitation of this dissertation is its inability to pinpoint the mechanisms through which the early childhood home and family environment influence school-based adaptive functioning. This limitation is especially salient as it pertains to the positive influence of negative life events. While possible mechanisms are presented (i.e., the possibility that some life events are accompanied by adaptive processes, including the receipt of social support) the results from this dissertation can neither support nor refute these potential processes.

Clearly, future research is needed in order to develop the interventions that will be most effective at enhancing mother-child interaction so that it facilitates growth in school-based adaptive functioning. This task is made additionally complex by theory and research indicating that optimal patterns of mother-child interaction differ according to children's disability diagnoses (Guralnick, 1999; Ly \& Hodapp, 2005). For example, one study found that mothers of children with Prader-Willi syndrome exhibited less directiveness during a problem solving task than mothers of children with Williams syndrome (Ly \& Hodapp, 2005). The authors interpreted this discrepancy to reflect an adaptive response to the higher levels of assistance required by the children with Williams syndrome (Ly \& Hodapp, 2005). In summary, then, future research needs to determine (1) specific elements of mother-child interaction that facilitate school-based 
adaptive functioning, (2) how these elements might differ according to child and / or family characteristics, with child characteristics including disability diagnosis, and (3) the interventions that are most likely to promote these optimal patterns of mother-child interaction.

Given the empirical support for the importance of mother-child interaction during early childhood, an additional limitation relates to potential interpretations in which mothers, and not larger socio-political forces, are blamed for poor developmental outcomes (Knight, 2013). Knight (2013) describes current trends in research in intellectual disabilities and concludes, "This body of research on the whole appears to be moving along a trajectory that places undue importance on the internal characteristics of families (in particular mothers) to cope with the pressures of managing a child with disability... at the expense of addressing systemic problems at the socio-political level" (Knight, 2013, p. 670). These systemic problems, including the current financial climate and related lack of service availability, should not be ignored (Knight, 2013). Relatedly, children have other relationships within the family that may be salient predictors of developmental outcomes. This dissertation did not include a measure of father-child interaction. It also did not include a measure of the quality of children's interactions with other primary caregivers and immediate family members, including siblings.

Finally, the inability to generalize findings to other samples is an important limitation of this dissertation. As described, most of the participants were middle class and of Euro-American descent. While this is representative of the population of individuals receiving EI in Massachusetts and New Hampshire at the time of study enrollment, the current sample represents a relatively homogenous one. It is important to 
acknowledge that patterns of mother-child interaction vary across cultures (Moscardino, Bertelli, \& Altoe, 2011) and their influences on child development likely vary accordingly.

Contributions. This dissertation contributes to the knowledge base about adaptive functioning in the classroom context for children with DD. While a body of research has investigated trajectories of adaptive functioning as reported by parents (Anderson et al., 2009; Baghdadli et al., 2012; Dykens et al., 2006; Hauser-Cram et al., 1999; Hauser-Cram et al., 2001; Vos et al., 2013), very little is known about trajectories of adaptive behavior displayed in contexts in which parents are less primary figures, such as the school. Due to the capacity of the environment to enhance or interfere with adaptive functioning (Harrison \& Boney, 2002), it is important to understand how trajectories of adaptive functioning operate in multiple contexts. The school is a particularly important context to consider, because meaningful participation in schoolbased activities can help children acquire skills that will contribute to social, vocational, and other achievements. Therefore, this dissertation provides much-needed depictions of school-based trajectories of adaptive functioning.

Also, almost all studies that have identified environmental predictors of adaptive functioning have utilized a parent-report measure (Anderson et al., 2009; Baghdadli et al., 2012; Dieterich et al., 2004; Fenning \& Baker, 2012; Hauser-Cram et al., 1999; HauserCram et al., 2001; Warfield, 1994). This dissertation identifies early childhood home and family predictors of school-based adaptive functioning. As such, it indicates elements of the early childhood home and family that should be targeted through intervention in order to enhance adaptive functioning outside of the home, in the classroom setting. 
Specifically, the observed salience of mother-child interaction has important implications for early intervention policy makers and providers. An important task for future research is to identify specific elements of mother-child interaction that contribute to positive development of school-based adaptive functioning. Also, while research indicates that interventions can potentially enhance the quality of interaction between mothers and their young children with DD (Kim \& Mahoney, 2005; Mahoney \& Perales, 2005), more research is needed in order to identify and / or develop interventions that target relevant interaction characteristics. 


\section{References}

Abidin, R. R. (1995). Parenting Stress Index manual (3rd ed.). Odessa, FL: Psychological Assessment Resources.

Achenbach, T. M., \& Edelbrock, C. S. (1983) Manual for the child behavior checklist and revised behavior profile. Burlington, VT: University of Vermont.

Ainsworth, M.D.S., Blehar, M., Waters, E., \& Wall, S. (1978). Patterns of attachment: A psychological study of the strange situation. Hillsdale, New Jersey: Erlbaum.

Aldao, A., \& Nolen-Hoeksema, S. (2012). The influence of context on the implementation of adaptive emotion regulation strategies. Behaviour Research and Therapy, 50(7-8), 493- 501. doi: 10.1016/j.brat.2012.04.004

American Psychiatric Association. (2013). Diagnostic and statistical manual of mental disorders $\left(5^{\text {th }}\right.$ ed.). Arlington, VA: American Psychiatric Publishing.

Anderson, D.K., Oti, R.S., Lord, C., \& Welch, K. (2009). Patterns of growth in adaptive social abilities among children with autism spectrum disorders. Journal of Abnormal Child Psychology, 37(7), 1019-1034. doi: 10.1007/s10802-009-93260.

Aram, D., \& Aviram, S. (2009). Mothers' storybook reading and kindergartners' socioemotional literacy development. Reading Psychology, 30(2), 175-194. doi: $10.1080 / 02702710802275348$

Arias, B., Verdugo, M.A., Navas, P., \& Gomez, L.E. (2013). Factor structure of the construct of adaptive behavior in children with and without intellectual disability. International Journal of Clinical Health Psychology, 13(2), 155-166. doi: $10.1016 / \mathrm{S} 1697-2600(13) 70019-\mathrm{X}$ 
Ayres, K., \& Cihak, D. (2010). Computer- and video-based instruction of foodpreparation skills: Acquisition, generalization, and maintenance. Intellectual and Developmental Disabilities, 48(3), 195-208. doi: 10.1352/1944-7558-48.3.195

Baghdadli, A., Assouline, B., Sonie, S., Pernon, E., Darrou, C., Michelon, C., ...\& Pry, R. (2012). Developmental trajectories of adaptive behaviors from early childhood to adolescence in a cohort of 152 children with autism spectrum disorders. Journal of Autism and Developmental Disorders, 42, 1314-1325. doi: $10.1007 / \mathrm{s} 10803-011-1357-\mathrm{z}$

Baker, J.K., Fenning, R.M., Crnic, K.A., Baker, B.L., \& Blacher, J. (2007). Prediction of social skills in 6-year-old children with and without developmental delays: Contributions of early regulation and maternal scaffolding. American Journal on Mental Retardation, 112(5), 375-391. doi: 10.1352/08958017(2007)112[0375:POSSIY]2.

Baker, C.E., \& Iruka, I.U. (2013). Maternal psychological functioning and children's school readiness: The mediating role of home environments for African American children. Early Childhood Research Quarterly, 28(3), 509-519. doi: 10.1016/j.ecresq.2013.02.004

Barnard, K. E. (1978). NCAST: Nursing Child Assessment Teaching Scale. Seattle: University of Washington Barnard, K.E., \& Kelly, J.F. (1990). Assessment of parent-child interaction. In S.J. Meisels \& J.P. Shonkoff (Eds.), Handbook of early childhood intervention (pp. 278-302). New York, New York: Cambridge University Press. 
Ben-Itzchak, E., Watson, L.R., \& Zachor, D.A. (2014). Cognitive ability is associated with different outcome trajectories in autism spectrum disorders. Journal of Autism and Developmental Disorders, 44(7). doi: 10.1007/s10803-014-2091-0

Bickel, R. (2007). Multilevel analysis for applied research: It's just regression! New York, NY, and London, England: The Guilford Press.

Bornstein, M.H. (In press). Children and their parents. In M.H. Bornstein and T. Leventhal (Eds.), Handbook of Child Psychology and Developmental Science $\left(7^{\text {th }}\right.$ ed.), Volume 4: Ecological Settings and Processes in Developmental Systems. Editor-in-chief: R.M. Lerner. Hoboken, NJ: Wiley.

Bornstein, M.H., Hahn, C., \& Suwalsky, J.T.D. (2013). Developmental pathways among adaptive functioning and externalizing and internalizing behavior problems: Cascades from childhood into adolescence. Applied Developmental Science, 17(2), 76-87. doi: 10.1080/10888691.2013.774875

Bornstein, M.H. \& Tamis-LeMonda, C.S. (1989). Maternal responsiveness and cognitive development in children. New Directions for Child Development, 43, 49-61. doi: $10.1002 / \mathrm{cd} .23219894306$

Bostrom, P.K., Broberg, M., \& Bodin, L. (2011). Child's positive and negative impacts on parents: A person-oriented approach to understanding temperament in preschool children with intellectual disabilities. Research in Developmental Disabilities, 32(5), 1860-1871. doi: 10.1016/j.ridd.2011.03.017

Bowlby, J. (1969). Attachment and loss, Vol. 1: Attachment (2nd ed.). New York, NY: Basic Books.

Bowlby, J. (1973). Separation. New York: Basic. 
Bradley R.H. (2002). Environment and parenting. In M.H. Bornstein (Ed.), Handbook of parenting ( $2^{\text {nd }}$ ed.) (Vol. 2) (pp. 281-314). Mahwah, New Jersey: Lawrence Erlbaum Associates.

Bradley, R.H., \& Caldwell, B.M. (1982). The consistency of the home environment and its relation to child development. International Journal of Behavioral Development, 5(4), 445-465.

Bradley, R.H., \& Caldwell, B.M. (1984). The HOME inventory and family demographics. Developmental Psychology, 20(2), 315-320. doi: 10.1037/00121649.20.2.315

Bradley, R.H., Caldwell, B.M., Rock, S.L., \& Harris, P.T. (1986). Early home environment and the development of competence: Findings from the Little Rock longitudinal study. Children's Environments Quarterly, 3(1), 10-22.

Bradley, R.H., \& Corwyn, R.F. (2004). "Family process" investments that matter for child well-being. In A. Kalil \& T. DeLeire (Eds.), Family investments in children's potential: Resources and parenting behaviors that promote success (pp. 1-32). Mahwah, New Jersey: Lawrence Erlbaum Associates Publishers.

Bradley, R.H., Rock, S.L., Whiteside, L., Caldwell, B.M., \& Brisby, J. (1991). Dimensions of parenting in families having children with disabilities. Exceptionality, 2(1), 41-61. doi: 10.1080/09362839109524765

Brady, N.C., Marquis, J., Fleming, K., \& McLean, L. (2004). Prelinguistic predictors of language growth in children with developmental disabilities. Journal of Speech, Language, \& Hearing Research, 47(3), 663-677. doi: 10.1044/1092$4388(2004 / 051$ 
Bronfenbrenner, U., \& Morris, P.A. (2006). The bioecological model of human development. In R.M. Lerner \& W. Damon (Eds.), Handbook of child psychology (6 ${ }^{\text {th }}$ ed.) (Vol. 1) (pp. 793-828). Hoboken, New Jersey: John Wiley \& Sons Inc.

Brooks-Gunn, J., \& Lewis, M. (1984). Maternal responsivity in interactions with handicapped infants. Child Development, 55(3), 782-793. doi: 10.2307/1130129

Bruininks, R.H., Woodcock, R., Weatherman, R., \& Hill, B.K. (1996). Scales of Independent Behavior - Revised. Park Allen, TX: DLM Teaching Resources.

Bugenthal, D.B., \& Grusec, J.E. (2006). Socialization processes. In N. Eisenberg (Vol Ed.), Handbook of child psychology: Vol. 3. Social, emotional, and personality development (pp. 366-428). New York, New York: Wiley.

Burbidge, J., Minnes, P., Buell, K., \& Ouellette-Kuntz, H. (2008). Preparing to leave school:Involvement of students with intellectual disabilities in productive activities. Journal on Developmental Disabilities, 14(1), 19-26.

Caldwell, B.M., \& Bradley, R.H. (1984). Home observation for measurement of the environment. Little Rock, Arkansas: University of Arkansas at Little Rock.

Chandler, R., \& Scott, M. (2011). Statistical methods for trend detection and analysis in the environmental sciences. Hoboken, NJ: John Wiley \& Sons.

Corrice, A.M., \& Glidden, L.M. (2009). The Down syndrome advantage: Fact or fiction? American Journal on Intellectual and Developmental Disabilities, 114, 254-268. doi: 10.1352/1994-7558-114.4.254-268

Cox, M.J., \& Paley, B. (2003). Understanding families as systems. Current Directions in Psychological Science, 12(5), 193-196. doi: 10.1111/1467-8721.01259 
de Bildt, A., Sytema, S., Kraijer, D., Sparrow, S., \& Minderaa, R. (2005).

Adaptive functioning and behaviour problems in relation to level of education in children and adolescents with intellectual disability. Journal of Intellectual Disability Research, 49(9), 672-681. doi: 10.1111/j.1365-2788.2005.00711.x

Dieterich, S.E., Hebert, H.M., Landry, S.H., Swank, P.R., \& Smith, K.E. (2004).

Maternal and child characteristics that influence the growth of daily living skills from infancy to school age in preterm and term children. Early Education \& Development, 15(3), 283-304. doi: 10.1207/s15566935eed1503_3

Doan, S.N., \& Evans, G.W. (2011). Maternal responsiveness moderates the relationship between allostatic load and working memory. Development and Psychopathology, 23, 873-880. doi: 10.1017/S0954579411000368

Dunst, C.J., Trivette, C.M., \& Cross, A.H. (1986). Mediating influences of social support: Personal, family, and child outcomes. American Journal of Mental Deficiency, 90(4), 403-417.

Dykens, E.M., Hodapp, R.M., \& Evans, D.W. (2006). Profiles and development of adaptive behavior in children with Down syndrome. Down Syndrome: Research and Practice, 9(3), 45-50. doi: 10.3104/reprints.293

Edelbroch, C., \& Achenbach, T.M. (1984). The teacher version of the Child Behavior Profile: I. Boys aged 6-11. Journal of Consulting and Clinical Psychology, 52(2), 207-217. doi: 10.1037/0022-006X.52.2.207

Eisenhower, A.S., Blacher, J., \& Bush, H.H. (2015). Longitudinal associations between externalizing problems and student-teacher relationship quality for young children 
with ASD. Research in Autism Spectrum Disorders, 9, 163-173. doi:

10.1016/j.rasd.2014.09.007

Evans, G.W., Schamberg, M.A., \& McEwen, B.S. (2009). Childhood poverty, chronic stress, and adult working memory. Proceedings of the National Academy of Sciences of the United States of America, 106(16), 6545-6549. doi: 10.1073/pnas.0811910106

Fenning, R.M., \& Baker, J.K. (2012). Mother-child interaction and resilience in children with early developmental risk. Journal of Family Psychology, 26(3), 411-420. doi: $10.1037 / \mathrm{a} 0028287$

Flanagan, H.E., Perry, A., \& Freeman, N.L. (2012). Effectiveness of large-scale community-based intensive Behavioral Intervention: A waitlist comparison study exploring outcomes and predictors. Research in Autism Spectrum Disorders, 6(2), 673-682. doi: 10.1016/j.rasd.2011.09.011

Fonteine, H., Zijlstra, H.P., \& Vlaskamp, C. (2008). Transfer of information between parents and teachers of children with profound intellectual and multiple disabilities at special educational centres. Journal of Applied Research in Intellectual Disabilities, 21(5), 477- 483. doi: 10.1111/j.1468-3148.2007.00415.x

Fraley, R.C., Roisman, G.I., \& Haltigan, J.D. (2013). The legacy of early experiences in development: Formalizing alternative models of how early experiences are carried forward over time. Developmental Psychology, 49(1), 109-126. doi: $10.1037 / \mathrm{a} 0027852$

Gilman, R., \& Anderman, E.M. (2006). The relationship between relative levels of motivation and intrapersonal, interpersonal, and academic functioning among 
older adolescents. Journal of School Psychology, 44(5), 375-391. doi:

10.1016/j.jsp.2006.03.004

Gilmore, L., Cuskelly, M., Jobling, A., \& Hayes, A. (2009). Maternal support for autonomy: Relationships with persistence for children with Down syndrome and typically developing children. Research in Developmental Disabilities, 30(5), 1023-1033. doi: 10.1016/j.ridd.2009.02.005

Gligorovic, M., \& Durovic, N.B. (2014). Inhibitory control and adaptive behaviour in children with mild intellectual disability. Journal of Intellectual Disability Research, 58(3), 233- 242. doi: 10.1111/jir.12000

Gray, P.H., Edwards, D.M., O’Callaghan, M.J., Cuskelly, M., \& Gibbons, K. (2013). Parenting stress in mothers of very preterm infants: Influence of development, temperament and maternal depression. Early Human Development, 89(9), 652629. doi: 10.1016/j.earlhumdev.2013.04.005

Green, S.E. (2007). "We're tired, not sad": Benefits and burdens of mothering a child with a disability. Social Science Medicine, 64, 150-163.

Green, V.A., Drysdale, H., Boelema, T., Smart, E., van der Meer, L., Achmadi, D., ...\& Lancioni, G. (2013). Use of video modeling to increase positive peer interactions of four preschool children with social skills difficulties. Education \& Treatment of Children, 36(2), 59-85.

Gabriels, R.L., Ivers, B.J., Hill, D.E., Agnew, J.A., \& McNeill, J. (2007). Stability of adaptive behaviors in middle-school children with autism spectrum disorders. Research in Autism Spectrum Disorders, 1(4), 291-303. doi:

10.1016/j.rasd.2006.11.004 
Grusec, J.E., \& Goodnow, J.J. (1994). Impact of parental discipline methods on the child's internalization of values: A reconceptualization of current points of view. Developmental Psychology, 30(1), 4-19. doi: 10.1037/0012-1649.30.1.4

Grusec, J.E., Goodnow, J.J., \& Kuczynski, L. (2000). New directions in analyses of parenting contributions to children's acquisition of values. Child Development, 71(1), 205-211. doi: 10.1111/1467-8624.00135

Guralnick, M.J. (1999). Family and child influences on the peer-related social competence of young children with developmental delays. Mental Retardation and Developmental Disabilities Research Reviews, 5(1), 21-29. doi: 10.1002/(SICI)1098-2779(1999)5:1

Guralnick, M.J., Neville, B., Connor, R.T., \& Hammond, M.A. (2003). Family factors associated with the peer social competence of young children with mild delays. American Journal on Mental Retardation, 108(4), 272-287. doi: 10.1352/0895$8017(2003) 108$

Harris, S., Kasari, C., \& Sigman, M.D. (1996). Joint attention and language gains in children with Down syndrome. American Journal on Mental Retardation, 100(6), 608-619.

Harrison, P.L., \& Boney, T.L. (2002). Best practices in the assessment of adaptive behavior. In A. Thomas \& J. Grimes (Eds.), Best practices in school psychology IV (1167-1179) (Vols. 1-2). Washington, DC: National Association of School Psychologists.

Harrison, P.L., \& Oakland, T. (2003). Adaptive behavior assessment system-Second Edition. San Antonio, TX: Harcourt Assessment. 
Hauser-Cram, P., Warfield, M. E., Shonkoff, J. P., \& Krauss, M. W. with Sayer, A., \& Upshur, C. C. (2001). Children with disabilities: A longitudinal study of child development and parent well-being. Monographs of the Society for Research in Child Development, 66 (3, Serial No. 266).

Hauser-Cram, P., Warfield, M.E., Shonkoff, J.P., Krauss, M.W., Upshur, C.C., \& Sayer, (1999). Family influences on adaptive development in young children with Down syndrome. Child Development, 70(4), 979-989. doi: 10.1111/1467-8624.00071

Hessl, D., Nguyen, D.V., Green, C., Chavez, A., Tassone, F., Hagerman, R.J.,...\& Hall, S. (2009). A solution to limitations of cognitive testing in children with intellectual disabilities: The case of fragile X syndrome. Journal of Neurodevelopmental Disorders, 1(1), 33-45. doi: 10.1007/s11689-008-9001-8

Heyman, M., \& Hauser-Cram, P. (2015). Negative life events predict performance on an executive function task in young adults with developmental disabilities. Journal of Intellectual Disability Research. doi: 10.1111/jir.12181

Hodapp, R.M., \& Ly, T.M. (2005). Parenting children with developmental disabilities. In T. Luster \& L. Okagaki (Eds.), Parenting: an ecological perspective ( $2^{\text {nd }}$ ed. $)$ (pp. 177-201). Mahwah, New Jersey: Erlbaum Associates Publishers.

Hoff, E. (2003). The specificity of environmental influence: Socioeconomic status affects early vocabulary development via maternal speech. Child Development, 74(5), 1368-1378. doi: 10.1111/1467-8624.00612

Hox, J. (2010). Multilevel analysis: Techniques and applications $\left(2^{\text {nd }}\right.$ ed.). New York, NY: Routledge. 
Hundert, J., Morrison, L., Mahoney, W., Mundy, F., \& Vernon, M.L. (1997). Parent and teacher assessments of the developmental status of children with severe, mild/moderate, or no developmental disabilities. Topics in Early Childhood Special Education, 17(4), 419- 434. doi: 10.1177/027112149701700404

Johnston, C.A., Steele, R.G., Herrera, E.A., \& Phipps, S. (2003). Parent and child reporting of negative life events: Discrepancy and agreement across pediatric samples. Journal of Pediatric Psychology, 28(8), 579-588.

Keogh, B.K. (1982). Temperament: An individual differences of importance in intervention programs. Topics in Early Childhood Special Education, 2(2), $25-$ 31.

Keogh, B.K., \& Burstein, N.D. (1988). Relationship of temperament to preschoolers' interactions with peers and teachers. Exceptional Children, 54(5), 456-461.

Kim, J.M., \& Mahoney, G. (2005). The effects of relationship focused intervention on Koreanparents and their young children with disabilities. Research in Developmental Disabilities, 26(2), 117-130. doi: 10.1016/j.ridd.2004.08.001

Knight, K. (2013). The changing face of the 'good mother': Trends in research into families with a child with intellectual disability, and some concerns. Disability \& Society, 28(5), 660-673. doi: 10.1080/09687599.2012.732540

Kohn, M. (1988). Kohn problem checklist / Kohn social competence scale: Manual (research edition). San Antonio, San Diego, Orlando, New York, Chicago, Toronto: The Psychological Corporation.

Lambert, N., Nihira, K., \& Leland, H. (1993). AAMR Adaptive Behavior Scales - School $(A B S-S: 2)$. Examiner's manual (2 ${ }^{\text {nd }}$ ed. $)$. Austin, TX: Pro-Ed. 
Landry, S.H., Smith, K.E., \& Swank, P.R. (2006). Responsive parenting: Establishing early foundations for social, communication, and independent problem-solving skills. Developmental Psychology, 42(4), 627-642. doi: 10.1037/00121649.42 .4 .627

Landry, S.H., Smith, K.E., Swank, P.R., \& Miller-Loncar, C.L. (2000). Early maternal and child influences on children's later independent cognitive and social functioning. Child Development, 71(2), 358-375. doi: 10.1111/1467-8624.00150

Lerner, J.V. (1993). The influence of child temperamental characteristics on parent behaviors. In T. Luster and L. Okagaki (Eds.), Parenting: An ecological perspective (pp. 101-120). Hillsdale, New Jersey: Lawrence Erlbaum Associates, Inc.

Lerner, R.M., Theokas, C., \& Jelicic, H. (2005). Youth as active agents in their own positive development: A developmental systems perspective. In R.M. Lerner, C. Theokas, \& H. Jelicic (Eds.), The adaptive self: Personal continuity and intentional self-development (pp. 31-47). Ashland, OH: Hogrefe \& Huber Publishers.

Leventhal, T., Dupere, V., \& Brooks-Gunn, J. (2009). Neighborhood influences on adolescent development. In. R.M. Lerner \& L. Steinberg (Eds.), Handbook of adolescent psychology (3rd ed.) (Vol. 2) (pp. 411-443). Hoboken, New Jersey: John Wiley \& Sons Inc.

Levine, S.C., Ratliff, K.R., Huttenlocher, J., \& Cannon, J. (2012). Early puzzle play: A predictor of preschoolers' spatial transformation skill. Developmental Psychology, 48(2), 530-542. doi: 10.1037/a0025913 
Lifter, K., Foster-Sanda, S., Arzamarski, C., Briesch, J., \& McClure, E. (2011).

Overview of play: Its uses and importance in early intervention / early childhood special education. Infants \& Young Children, 24(3), 225-245. doi:

10.1097/IYC.0b013e31821e995c

Lillie, T., \& Vakil, S. (2002). Transitions in early childhood for students with disabilities: Law and best practice. Early Childhood Education Journal, 30(1), 53-58. doi: 10.1023/A:1016598016500

Linver, M.R., Brooks-Gunn, J., \& Kohen, D.E. (2002). Family processes as pathways from income to young children's development. Developmental Psychology, 38(5), 719-734. doi: 10.1037/0012-1649.38.5.719

Ly, T.M., \& Hodapp, R.M. (2005). Children with Prader-Willi syndrome vs. Williams syndrome: Indirect effects on parents during a jigsaw puzzle task. Journal of Intellectual Disability Research, 49(12), 929-939. doi: 10.1111/j.13652788.2005.00782.x

Maccoby, E.E. (1992). The role of parents in the socialization of children: An historical overview. Developmental Psychology, 28(6), 1006-1017. doi: 10.1037/00121649.28.6.1006

Maenner, M.J., Smith, L.E., Hong, J., Makuch, R., Greenberg, J.S., \& Mailick, M.R. (2013). Evaluation of an activities of daily living scale for adolescents and adults with developmental disabilities. Disability and Health Journal, 6(1), 8-17. doi: 10.1016/j.dhjo.2012.08.005

Mahoney, G., \& Perales, F. (2005). Relationship-focused early intervention with children with pervasive developmental disorders and other disabilities: A 
comparative study. Journal of Developmental and Behavioral Pediatrics, 26(2), 77-85. doi: 10.1097/00004703-200504000-00002

Malone, D.G., \& Gallagher, P.A. (2008). Transition to preschool programs for young children with disabilities. Journal of Early Intervention, 30(4), 341-356. doi: $10.1177 / 1053815108321330$

Matheson, C., Olsen, R.J., \& Weisner, T. (2007). A good friend is hard to find: Friendship among adolescents with disabilities. American Journal on Mental Retardation, 112(5), 319-329. doi: 10.1352/08958017(2007)112[0319:AGFIHT]2.0.CO;2

McCartney, K., \& Rosenthal, R. (2000). Effect size, practical importance, and social policy for children. Child Development, 71(1), 173-180. doi: 10.1111/14678624.00131

McCarthy, D. (1972). Manual for the McCarthy Scales of Children's Abilities. San Antonio, TX: The Psychological Corporation.

McDuffie, A., \& Yoder, P. (2010). Types of parent verbal responsiveness that predict language in young children with autism spectrum disorder. Journal of Speech, Language, and Hearing Research, 53(4), 1026-1039. doi: 10.1044/10924388(2009/09-0023)

Miser, T.M., \& Hupp, J.M. (2012). The influence of socioeconomic status, home environment, and childcare on child language abilities. Current psychology: A Journal for diverse perspectives on diverse psychological issues, 31(2), 144-159. doi: 10.1007/s12144-012-9139-0 
Mitchell, D.B. \& Hauser-Cram, P. (2009). Early predictors of behavior problems: Two years after early intervention. Journal of Early Intervention, 32(1), 3-16. doi: $10.1177 / 1053815109349113$

Mitchell, D.B., \& Hauser-Cram, P. (2010). Early childhood predictors of mothers' and fathers' relationships with adolescents with developmental disabilities. Journal of Intellectual Disability Research, 54(6), 487-500. doi: 10.1111/j.13652788.2010.01268.x

Mitchell, S., Cardy, J.O., \& Zwaigenbaum, L. (2011). Differentiating autism spectrum disorder from other developmental delays in the first two years of life. Developmental Disabilities Research Reviews, 17(2), 130-140. doi: 10.1002/ddrr.1107

Mitchell, D.B., Hauser-Cram, P., \& Crossman, M.K. (2014). Relationship dimensions of the 'Down syndrome advantage'. Journal of Intellectual Disability Research. doi: $10.1111 /$ jir.12153

Moscardino, U., Bertelli, C., Altoe, G. (2011). Culture, migration and parenting: A comparative study of mother-infant interaction and childrearing patterns in Romanian, Romanian immigrant, and Italian families. International Journal of Developmental Science, 5(1-2), 11-25.

Murray, A.D., \& Hornbaker, A.V. (1997). Maternal directive and facilitative interaction styles: Associations with language and cognitive development of low risk and high risk toddlers. Development and Psychopathology, 9(3), 507-516. doi: $10.1017 /$ S0954579497001271 
O’Connor, E., Dearing, E., \& Collins, B. A. (2011). Teacher-child relationship and behavior problem trajectories in elementary school. American Educational Research Journal, 48, 120 -162.

Odom, S.L., Zercher, C., Li, S., Marquart, J.M., Sandall, S., \& Brown, W.H. (2006). Social acceptance and rejection of preschool children with disabilities: A mixedmethod analysis. Journal of Educational Psychology, 98(4), 807-823. doi: 10.1037/0022-0663.98.4.807

Parke, R.D., \& Buriel, R. (2006). Socialization in the family: Ethnic and ecological perspectives. In N. Eisenberg, W. Damon, \& R.M. Lerner (Eds.), Handbook of child psychology: Vol. 3, Social, emotional, and personality development ( $6^{\text {th }}$ ed.) (pp. 429-504). New Jersey, United States: John Wiley \& Sons Inc.

Parsons, T. (1959). The school class as a social system: Some of its functions in American society. Harvard Educational Review, 29(4), 298-318.

Peterson, C.A., Wall, S., Raikes, H.A., Kisker, E.E., Swanson, M.E., Jerald, J.,..\&\& Qiao, W. (2004). Early head start: Identifying and serving children with disabilities. Topics in Early Childhood Special Eduaction, 24(2), 76-88. doi: $10.1177 / 02711214040240020301$

Putnam, S.P., Sanson, A.V., \& Rothbart, M.K. (2002). Child temperament and parenting. In M. Bornstein (Ed.), Handbook of parenting: Vol. 1: Children and parenting ( $2^{\text {nd }}$ Edition $), \quad$ pp. 255-277. Mahwah, New Jersey: Lawrence Erlbaum Associates.

Purrazzella, K., \& Mechling, L. (2013). Use of an iPhone 4 with video features to assist location of students with moderate intellectual disability when lost in community 
settings. Education and Training in Autism and Developmental Disabilities, 48(2), 179-189.

Reed, M.L., \& Edelbrock, C. (1983). Reliability and validity of the Direct Observation Form of the Child Behavior Checklist. Journal of Abnormal Child Psychology, 0 11(4), 521-530. doi: 10.1007/BF00917081

Robinson-Cimpian, J.P., Lubienski, S.T., Ganley, C.M., \& Copur-Gencturk, Y. (2014). Teachers' perceptions of students' mathematics proficiency may exacerbate early gender gaps in achievement. Developmental Psychology, 50(4), 1262-1281. doi: $10.1037 / \mathrm{a} 0035073$

Roisman, G.I., \& Fraley, R.C. (2013). Developmental mechanisms underlying the legacy of childhood experiences. Child Development Perspectives, 7(3), 149-154. doi: $10.1111 /$ cdep. 12030

Ron, P. (2006). Care giving offspring to aging parents: How it affects their marital relations, parenthood, and mental health. Illness, Crisis, \& Loss, 14(1), 1-21.

Rousey, A.M., Wild, M., \& Blacher, J. (2002). Stability of measures of the home environment for families of children with severe disabilities. Research in Developmental Disabilities, 23(1), 17-35. doi: 10.1016/S0891-4222(01)00089-0

Sameroff, A.J. (1995). General systems theory and developmental psychopathology. In D. Cicchetti \& D.J. Cohen (Eds.), Developmental psychopathology: Vol. 1. Theory and methods (pp. 659-695). New York, New York: Wiley. 
Schaefer, E.S., \& Aaronson, M.R. (1966). Classroom behavior inventory: Preschool to primary. Unpublished manuscript. (Available from School of Public Health, University of North Carolina, Chapel Hill, NC.)

Shonkoff, J.P. (2009). Leveraging the biology of adversity to address the roots of disparities in health and development. Proceedings of the National Academy of Sciences of the United States of America, 109(2), 17302-17307. doi: 10.1073/pnas.1121259109

Shonkoff, J.P., Garner, A.S., Siegel, B.S., Dobbins, M.I., Earls, M.F., Garner, A.S., ...\& Wood, D.L. (2012). The lifelong effects of early childhood adversity and toxic stress. Pediatrics, 129, e232-e246. doi: 10.1542/peds.2011-2663

Siddiqui, O., Flay, B.R., \& Hu, F.B. (1996). Factors affecting attrition in a longitudinal smoking prevention study. Preventive Medicine: An International Journal Devoted to Practice and Theory, 25(5), 554-560. doi: 10.1006/pmed.1996.0089

Smith, L.E., Maenner, M.J., \& Seltzer, M.M. (2012). Developmental trajectories in adolescents and adults with autism: The case of daily living skills. Journal of the American Academy of Child \& Adolescent Psychiatry, 51(6), 622-631. doi: 10.1016/j.jaac.2012.03.001

Sparrow, S.S., Balla, D.A., \& Cicchetti, D.V. (1984). Vineland Adaptive Behavior Scales survey form manual (Interview edition). Circle Pines, Minnesota: American Guidance Service.

Sparrow, S.S., Balla, D. A., \& Cicchetti, D. V. (1985). Vineland Adaptive Behavior Scales: Classroom edition manual. Circle Pines, MN: American Guidance Service. 
Sparrow, S., Cicchetti, D., \& Balla, D. (2005). Vineland Adaptive Behavior Scales, Second Edition (Vineland-II). Circle Pines, Minnesota: American Guidance Service.

Sroufe, L.A., Egeland, B., \& Kreutzer, T. (1990). The fate of early experience following developmental change: Longitudinal approaches to individual adaptation in childhood. Child Development, 61(5), 1363-1373. doi: 10.2307/1130748

Stock, J.H., \& Watson, M.W. (2007). Why has U.S. inflation become harder to forecast? Journal of Money, Credit and Banking, 39(1), 3-34.

Sumner, G., \& Spietz, A. (1994). NCAST caregiver/parent-child interaction teaching manual. Seattle, WA: NCAST Publications, University of Washington, School of Nursing.

Tasse, M.J., Schalock, R.L., Balboni, G., Bersani, H., Borthwick-Duffy, S.A., Scott, S., ... \& Zhang, D. (2012). The construct of adaptive behavior: Its conceptualization, measurement, and use in the field of intellectual disability. American Journal on Intellectual and Developmental Disabilities, 117(4), 291303. doi: 10.1352/1944-7558-117.4.291

Thorndike, R., Hagen, E., \& Sattler, J. (1986). Stanford-Binet intelligence scale (4 ${ }^{\text {th }}$ ed.). Chicago: The Riverside Publishing Company.

Trivette, C.M., Dunst, C.J., \& Hamby, D.W. (2010). Influences of family-systems intervention practices on parent-child interactions and child development. Topics in Early Childhood Special Education, 30(1), 3-19. doi:

$10.1177 / 0271121410364250$ 
Tyler, L.E. (1984). What tests don't measure. Journal of Counseling \& Development, 63(1), 48-50. doi: 10.1002/j.1556-6676.1984.tb02682.x

United States Census Bureau (2012). Money income of households - median income by race and Hispanic origin, in current and constant (2009) dollars: 1980 to 2009. Retrieved from http://www.census.gov/compendia/statab/2012/tables/12s0691.pdf United States Department of Education (2014). Early intervention program for infants and toddlers with disabilities. Retrieved from http://www2.ed.gov/programs/osepeip/index.html

Van Duijn, G., Dijkxhoorn, Y., Scholte, E.M., van Berckelaer-Onnes, I.A. (2010). The development of adaptive skills in young people with Down syndrome. Journal of Intellectual Disability Research, 54(11), 943-954. doi: 10.1111/j.13652788.2010.01316.x

VanMeter, L., Fein, D., Morris, R., Waterhouse, L., \& Allen, D. (1997). Delay versus deviance in autistic social behavior. Journal of Autism and Developmental Disorders, 27(5), 557-569. doi: 10.1023/A:1025830110640

Verschueren, K., Doumen, S., \& Buyse, E. (2012). Relationships with mother, teacher, and peers: Unique and joint effects on young children's self-concept. Attachment \& Human Development, 14(3), 233-248. doi: 10.1080/14616734.2012.672263

Voelker, S.L., Johnston, T.C., Agar, C.G., Gragg, M., \& Menna, R. (2008). Diagnostic inventory for screening children (DISC): Evidence of concurrent validity in a preschool rehabilitation setting. Journal on Developmental Disabilities, 14(2), 69-78. 
Voelker, S., Shore, D., Hakim-Larson, J., \& Bruner, D. (1997). Discrepancies in parent and teacher ratings of adaptive behavior of children with multiple disabilities. Mental Retardation, 35(1), 10-17. doi: 10.1352/0047-6765(1997)035

Voelker, S.L., Shore, D.L., Lee, C.H., \& Szuszkiewicz, T.A. (2000). Congruence in parent and teacher ratings of adaptive behavior of low-functioning children. Journal of Developmental and Physical Disabilities, 12(4), 367-376. doi: 10.1023/A:1009436230984

Vos, R.C., Becher, J.G., Ketelaar, M., Smits, D., Voorman, J.M., Tan, S.S., .. \& \& Dallmeijer, A.J. (2013). Developmental trajectories of daily activities in children and adolescents with cerebral palsy. Pediatrics, 132(4), e915-e923. doi: 10.1542/peds.2013-0499

Vygotsky, L.S. (1978). Mind in society. Cambridge, Massachusetts: Harvard University Press.

Wang, Y., \& Dix, T. (2013). Patterns of depressive parenting: Why they occur and their role in early developmental risk. Journal of Family Psychology, 27(6), 884-895. doi: $10.1037 / \mathrm{a} 0034829$

Warfield, M.E. (1994). A cost-effectiveness analysis of early intervention services in Massachusetts: Implications for policy. Educational Evaluation and Policy Analysis, 16(1), 87-99. doi: 10.2307/1164385

Warfield, M.E., Krauss, M.W., Hauser-Cram, P., Upshur, C.C., \& Shonkoff, J.P. (1999). Adaptation during early childhood among mothers of children with disabilities. Journal of Developmental and Behavioral Pediatrics, 20(1), 9-16. doi: 10.1097/00004703-199902000-00002 
Warren, S.F., Brady, N., Sterling, A., Fleming, K., \& Marquis, J. (2010). Maternal responsivity predicts language development in young children with fragile $\mathrm{X}$ syndrome. American Journal on Intellectual and Developmental Disabilities, 115(1), 54-75. doi: 10.1352/1944-7558-115.1.54

West, K.K., Mathews, B.L., \& Kerns, K.A. (2013). Mother-child attachment and cognitive performance in middle childhood: An examination of mediating mechanisms. Early Childhood Research Quarterly, 28(2), 259-270. doi: 10.1016//j.ecresq.2012.07.005

Widaman, K. F. (2006). Best practices in quantitative methods for developmentalists: Missing data: What to do with or without them. Monographs of the Society for Research in Child Development, 71(3), 42-64. doi: 10.1111/j.15405834.2006.00404.x

Widaman, K.F., \& McGrew, K.S. (1996). The structure of adaptive behavior. In J.W. Jacobson \& J.S. Mulick (Eds.), Manual of diagnosis and professional practice in mental retardation (pp. 97-110). Washington, DC: American Psychological Association.

Wieland, N., Green, S., Ellingsen, R., \& Baker, B.L. (2014). Parent-child problem solving in families of children with or without intellectual disability. Journal of Intellectual Disability Research, 58(1), 17-30. doi: 10.1111/jir.12009

Woodman, A.C. (2014). Trajectories of stress among parents of children with disabilities: A dyadic analysis. Family Relations: An Interdisciplinary Journal of Applied Family Studies, 63(1), 39-54. doi: 10.1111/fare.12049 
Young, J.M., \& Hauser-Cram, P. (2006). Mother-child interaction as a predictor of mastery motivation in children with disabilities born preterm. Journal of Early Intervention, 28(4), 252-263. doi: 10.1177/105381510602800402 
Table 1

Family and Child Demographic Characteristics at T3 $(N=170)$

\begin{tabular}{|c|c|c|}
\hline & $\%$ & Mean (SD) \\
\hline \multicolumn{3}{|l|}{ Child } \\
\hline \multicolumn{3}{|l|}{ Type of disability } \\
\hline Down syndrome & $30.6 \%$ & \\
\hline Motor impairment & $39.4 \%$ & \\
\hline Developmental delay of unknown etiology & $30.0 \%$ & \\
\hline \multicolumn{3}{|l|}{ Gender } \\
\hline Male & $54.7 \%$ & \\
\hline \multicolumn{3}{|l|}{ Racial/ethnic origin } \\
\hline European American & $91.5 \%$ & \\
\hline African American & $1.4 \%$ & \\
\hline Hispanic & $4.2 \%$ & \\
\hline Mixed race/other & $2.8 \%$ & \\
\hline Lived with both parents & $81.9 \%$ & \\
\hline Lived with mother only & $15.7 \%$ & \\
\hline \multicolumn{3}{|l|}{ Family } \\
\hline \multicolumn{3}{|l|}{ Income (1989-1991) } \\
\hline Less than $20 \mathrm{~K}$ & $25.6 \%$ & \\
\hline Between $20 \mathrm{~K}$ and $30 \mathrm{~K}$ & $23.3 \%$ & \\
\hline Between $30 \mathrm{~K}$ and $40 \mathrm{~K}$ & $19.7 \%$ & \\
\hline More than $40 \mathrm{~K}$ & $31.3 \%$ & \\
\hline Number of additional children & & $1.22(1.02)$ \\
\hline
\end{tabular}


Table 1 (Cont.)

\begin{tabular}{lll}
\hline & $\%$ & Mean (SD) \\
\hline Mother & $81.7 \%$ & \\
Marital status (married) & $46.7 \%$ & $13.85(2.42)$ \\
Employment status (employed) & & $31.59(5.13)$ \\
Education (years) & & \\
Age (years) & $84.6 \%$ & \\
Father & $92.9 \%$ & $13.99(3.02)$ \\
Marital status (married) & & 33.87 (5.86) \\
Employment status (employed) & & \\
Education (years) & & \\
Age (years)
\end{tabular}


Table 2

Teacher Characteristics at Multiple Time Points

\begin{tabular}{|c|c|c|c|c|c|}
\hline & T3 & T5 & $\mathrm{T} 8$ & $\mathrm{~T} 10$ & T15 \\
\hline \multicolumn{6}{|l|}{ Level of education- $\%$} \\
\hline Bachelors & $50.7 \%$ & $51.4 \%$ & $51.2 \%$ & $32.8 \%$ & \\
\hline Masters & $41.1 \%$ & $38.5 \%$ & $36.2 \%$ & $46.6 \%$ & \\
\hline Other & $8.2 \%$ & $10.2 \%$ & $12.6 \%$ & $12.2 \%$ & \\
\hline \multicolumn{6}{|l|}{ Special education degree - \% } \\
\hline Yes & $75.9 \%$ & $70.3 \%$ & $45.7 \%$ & $55.7 \%$ & \\
\hline \multicolumn{6}{|l|}{ Role - \% } \\
\hline Subject teacher & & & & & $9.0 \%$ \\
\hline Special education teacher & & & & & $65.4 \%$ \\
\hline $\begin{array}{l}\text { Both subject and special } \\
\text { education teacher }\end{array}$ & & & & & $9.0 \%$ \\
\hline Aide / assistant & & & & & $7.7 \%$ \\
\hline Other & & & & & $9.0 \%$ \\
\hline \multicolumn{6}{|l|}{ Gender - \% } \\
\hline Female & $97.8 \%$ & $97.3 \%$ & & & \\
\hline $\begin{array}{l}\text { Years of experience in current } \\
\text { position - Mean (SD) }\end{array}$ & $\begin{array}{l}6.68 \\
(6.08)\end{array}$ & $\begin{array}{l}6.80 \\
(5.22)\end{array}$ & $\begin{array}{l}15.25 \\
(9.66)\end{array}$ & $\begin{array}{l}13.73 \\
(8.62)\end{array}$ & \\
\hline $\mathrm{N}$ & 146 & 148 & 127 & 131 & 78 \\
\hline
\end{tabular}


Table 3

List of Measures

\begin{tabular}{|c|c|c|c|c|}
\hline Construct & Measure & Reporter & $\begin{array}{c}\text { Time } \\
\text { Points }\end{array}$ & $\begin{array}{c}\text { Cronbach's } \\
\text { Alpha }\end{array}$ \\
\hline $\begin{array}{l}\text { Mother } \\
\text { educational } \\
\text { attainment }\end{array}$ & Demographic questionnaire & Mother & 3 & $\mathrm{n} / \mathrm{a}$ \\
\hline Family income & Demographic questionnaire & Mother & 3 & $\mathrm{n} / \mathrm{a}$ \\
\hline Child gender & Demographic questionnaire & Mother & 3 & $\mathrm{n} / \mathrm{a}$ \\
\hline $\begin{array}{l}\text { Child type of } \\
\text { disability }\end{array}$ & Demographic questionnaire & Mother & 1 & $\mathrm{n} / \mathrm{a}$ \\
\hline $\begin{array}{l}\text { Home } \\
\text { environment }\end{array}$ & $\begin{array}{l}\text { Home Observation for the } \\
\text { Measurement of the } \\
\text { Environment (Bradley \& } \\
\text { Caldwell, 1984) }\end{array}$ & $\mathrm{n} / \mathrm{a}$ & 2 & 0.79 \\
\hline $\begin{array}{l}\text { Negative life } \\
\text { events }\end{array}$ & Life Events Scale (Abidin, 1995) & Mother & $1,2,3$ & $\mathrm{n} / \mathrm{a}$ \\
\hline $\begin{array}{l}\text { Child evocative } \\
\text { effects }\end{array}$ & $\begin{array}{l}\text { Parenting Stress Index (Abidin, } \\
\text { 1995) }\end{array}$ & $\begin{array}{l}\text { Mother and } \\
\text { father }\end{array}$ & 3 & $\begin{array}{l}0.86 \text { (father) } \\
-0.87 \\
\text { (mother) }\end{array}$ \\
\hline $\begin{array}{l}\text { Mother-child } \\
\text { interaction }\end{array}$ & $\begin{array}{l}\text { Nursing Child Assessment } \\
\text { Teaching Scale (Barnard, 1978) }\end{array}$ & $\mathrm{n} / \mathrm{a}$ & 3 & 0.82 \\
\hline $\begin{array}{l}\text { Classroom } \\
\text { placement type }\end{array}$ & Teacher questionnaire & Teacher & $3,5,8,10$ & $\mathrm{n} / \mathrm{a}$ \\
\hline
\end{tabular}


Table 3 Continued

List of Measures

\begin{tabular}{|c|c|c|c|c|}
\hline Construct & Measure & Reporter & $\begin{array}{l}\text { Time } \\
\text { Points }\end{array}$ & $\begin{array}{l}\text { Cronbach's } \\
\text { Alpha }\end{array}$ \\
\hline $\begin{array}{l}\text { Classroom } \\
\text { placement type }\end{array}$ & Mother interview & Mother & 15 & $\mathrm{n} / \mathrm{a}$ \\
\hline $\begin{array}{l}\text { Classroom } \\
\text { externalizing } \\
\text { behaviors }\end{array}$ & $\begin{array}{l}\text { Kohn Problem Checklist (Kohn, } \\
\text { 1988) }\end{array}$ & Teacher & 3,5 & $0.84-0.91$ \\
\hline $\begin{array}{l}\text { Classroom } \\
\text { externalizing } \\
\text { behaviors }\end{array}$ & $\begin{array}{c}\text { Child Behavior Checklist - } \\
\text { Teacher Version (Achenbach \& } \\
\text { Edelbrock, 1983) }\end{array}$ & Teacher & $8,10,15$ & $0.93->0.99$ \\
\hline $\begin{array}{l}\text { Child adaptive } \\
\text { functioning } \\
\text { (socialization) }\end{array}$ & $\begin{array}{l}\text { Vineland Adaptive Behavior } \\
\text { Scales-Classroom Edition } \\
\text { (Sparrow et al., 1985) }\end{array}$ & Teacher & $\begin{array}{l}3,5,8,10, \\
15\end{array}$ & $0.85-0.98$ \\
\hline $\begin{array}{l}\text { Child adaptive } \\
\text { functioning (daily } \\
\text { living skills) }\end{array}$ & $\begin{array}{l}\text { Vineland Adaptive Behavior } \\
\text { Scales-Classroom Edition } \\
\text { (Sparrow et al., 1985) }\end{array}$ & Teacher & $\begin{array}{l}3,5,8,10, \\
15\end{array}$ & $0.97-0.99$ \\
\hline $\begin{array}{l}\text { Child adaptive } \\
\text { functioning } \\
\text { (communication) }\end{array}$ & $\begin{array}{l}\text { Vineland Adaptive Behavior } \\
\text { Scales-Classroom Edition } \\
\text { (Sparrow et al., 1985) }\end{array}$ & Teacher & $\begin{array}{l}3,5,8,10, \\
15\end{array}$ & $0.98-0.99$ \\
\hline $\begin{array}{l}\text { Child adaptive } \\
\text { functioning (total } \\
\text { score) }\end{array}$ & $\begin{array}{l}\text { Vineland Adaptive Behavior } \\
\text { Scales-Classroom Edition } \\
\text { (Sparrow et al., 1985) }\end{array}$ & Teacher & $\begin{array}{l}3,5,8,10, \\
15\end{array}$ & $\geq 0.99$ \\
\hline
\end{tabular}


Table 4

Percentage of Missing Data: Child and family data $(N=170)$

\begin{tabular}{ll}
\hline \multicolumn{1}{c}{ Construct } & $\%$ Missing \\
\hline Family income & $4.1 \%$ \\
$\begin{array}{l}\text { Maternal educational } \\
\text { attainment } \\
\text { Child gender }\end{array}$ & $0 \%$ \\
Child type of disability & $0 \%$ \\
Child evocative effects & \\
\multicolumn{1}{c}{ Mother report } & $20 \%$ \\
Father report & $40 \%$ \\
Home environment & $1.2 \%$ \\
Negative life events & $2.4 \%$ \\
Mother-child interaction & $2.4 \%$ \\
\hline
\end{tabular}


Table 5

Percentage of Missing Data: Teacher and classroom measures (N=170)

\begin{tabular}{lccccc}
\hline \multicolumn{1}{c}{ Construct } & $\mathrm{T} 3$ & $\mathrm{~T} 5$ & $\mathrm{~T} 8$ & $\mathrm{~T} 10$ & $\mathrm{~T} 15$ \\
\hline $\begin{array}{l}\text { Classroom placement } \\
\text { type (substantially } \\
\text { separate vs. inclusive) }\end{array}$ & $28.8 \%$ & $24.1 \%$ & $25.9 \%$ & $25.3 \%$ & $26.5 \%$ \\
$\begin{array}{l}\text { Classroom } \\
\text { externalizing } \\
\text { behaviors }\end{array}$ & $18.1 \%$ & $17.5 \%$ & $30.5 \%$ & $27.1 \%$ & $55.9 \%$ \\
$\begin{array}{l}\text { Adaptive functioning } \\
\text { Socialization }\end{array}$ & $13.5 \%$ & $12.4 \%$ & $25.9 \%$ & $24.7 \%$ & $26.5 \%$ \\
$\begin{array}{l}\text { Daily living skills } \\
\text { Communication }\end{array}$ & $13.5 \%$ & $12.4 \%$ & $25.9 \%$ & $24.7 \%$ & $26.5 \%$ \\
$\begin{array}{l}\text { Total score } \\
\text { 13.5\% }\end{array}$ & $13.5 \%$ & $12.4 \%$ & $25.9 \%$ & $24 \%$ & \\
\hline
\end{tabular}


Table 6

Descriptive Statistics: Continuous Variables

\begin{tabular}{|c|c|c|c|}
\hline & Mean & Standard Deviation & Range \\
\hline $\begin{array}{l}\text { Mother's years of } \\
\text { education }\end{array}$ & 13.88 & 2.42 & $9-21$ \\
\hline Negative life events & 6.99 & 4.89 & $0-26$ \\
\hline Home environment & 38.58 & 4.60 & $23-44$ \\
\hline $\begin{array}{l}\text { Mother-child } \\
\text { interaction }\end{array}$ & 37.61 & 6.07 & $15-49$ \\
\hline Child evocative effects & & & \\
\hline $\begin{array}{l}\text { Adaptability (mother } \\
\text { report) }\end{array}$ & 26.00 & 6.31 & $12-44$ \\
\hline $\begin{array}{l}\text { Demandingness } \\
\text { (mother report) }\end{array}$ & 21.07 & 5.68 & $9-37$ \\
\hline Mood (mother report) & 9.73 & 3.08 & $5-18$ \\
\hline Mother report total & 56.79 & 13.26 & $27-89$ \\
\hline $\begin{array}{l}\text { Adaptability (father } \\
\text { report) }\end{array}$ & 27.63 & 6.09 & $13-43$ \\
\hline $\begin{array}{l}\text { Demandingness } \\
\text { (father report) }\end{array}$ & 21.35 & 5.50 & $10-26$ \\
\hline Mood (father report) & 10.20 & 2.78 & $5-19$ \\
\hline Father report total & 59.18 & 12.21 & $30-92$ \\
\hline $\begin{array}{l}\text { Total child evocative } \\
\text { effects (sum of mother } \\
\& \text { father } z \text {-scores) }\end{array}$ & 0.00 & 1.82 & $-4.41-4.68$ \\
\hline Classroom externalizin & iors & & \\
\hline Age 3 & 49.61 & 8.67 & $41-85$ \\
\hline Age 5 & 51.16 & 10.49 & $41-100$ \\
\hline Age 8 & 56.37 & 9.29 & $39-80$ \\
\hline Age 10 & 55.78 & 9.48 & $39-78$ \\
\hline Age 15 & 57.46 & 9.20 & $42-81$ \\
\hline
\end{tabular}


Table 6 Continued

Descriptive Statistics: Continuous Variables

\begin{tabular}{|c|c|c|c|}
\hline & Mean & Deviation & Range \\
\hline \multicolumn{4}{|c|}{$\begin{array}{l}\text { Adaptive functioning raw scores } \\
\text { (and corresponding age equivalents) }\end{array}$} \\
\hline Socialization (age 3) & 20.64 (1 yr. 7 mo.) & 11.44 & $0-68$ \\
\hline Communication (age 3) & 24.07 (1 yr. 5 mo.) & 17.16 & $0-75$ \\
\hline Daily living skills (age 3) & 23.61 (1 yr. 8 mo.) & 15.61 & $0-72$ \\
\hline Total (age 3) & 68.33 & 41.37 & $0-203$ \\
\hline Socialization (age 5) & 34.42 (2 yr. 6 mo.) & 19.09 & $0-90$ \\
\hline Communication (age 5) & 41.62 (2 yr. 4 mo.) & 23.66 & $0-92$ \\
\hline Daily living skills (age 5) & 49.70 (2 yr. 10 mo.) & 28.59 & $0-145$ \\
\hline Total (age 5) & 125.73 & 68.03 & $0-320$ \\
\hline Socialization (age 8) & 46.21 (3 yr. 5 mo.) & 23.98 & $2-100$ \\
\hline Communication (age 8) & 60.46 (3 yr. 8 mo. $)$ & 33.51 & $0-126$ \\
\hline Daily living skills (age 8) & 79.92 (4 yr. 4 mo.) & 44.12 & $0-175$ \\
\hline Total (age 8) & 186.60 & 97.92 & $2-381$ \\
\hline Socialization (age 10) & 53.23 (4 yr. 1 mo.) & 28.23 & $2-106$ \\
\hline Communication (age 10) & 71.22 (4 yr. 11 mo.) & 38.18 & $0-126$ \\
\hline Daily living skills (age 10) & 98.16 (5 yr. 4 mo.) & 53.87 & $0-193$ \\
\hline Total (age 10) & 222.60 & 117.74 & $2-416$ \\
\hline Socialization (age 15) & 78.91 (10 yr. 0 mo.) & 29.80 & $7-124$ \\
\hline Communication (age 15) & 89.06 (6 yr. 8 mo.) & 38.99 & $7-134$ \\
\hline Daily living skills (age 15) & 102.78 (5 yr. 8 mo.) & 48.79 & $0-174$ \\
\hline Total (age 15) & 270.64 & 115.10 & $16-428$ \\
\hline
\end{tabular}

Note. Corresponding age equivalent scores are in parentheses next to raw score means for the three domains (socialization, communication, daily living skills). Age equivalents are not available for total scores. 
Table 7

Descriptive Statistics: Categorical Variables

\begin{tabular}{ll}
\hline & Percent \\
\hline Diagnostic group & \\
Down syndrome & $30.6 \%$ \\
Motor impairment & $39.4 \%$ \\
Other DD & $30.0 \%$ \\
\hline Family income & \\
(1989-1991) & \\
Less than 20K & $25.6 \%$ \\
20K-30K & $23.3 \%$ \\
$30 K-40 K$ & $19.7 \%$ \\
More than $40 K$ & $31.3 \%$ \\
\hline Gender & $54.7 \%$ male \\
\hline Classroom & \\
characteristics & \\
Age 3 & $76.0 \%$ substantially separate \\
Age 5 & $55.0 \%$ substantially separate \\
Age 8 & $40.5 \%$ substantially separate \\
Age 10 & $48.8 \%$ substantially separate \\
Age 15 & $53.6 \%$ substantially separate \\
\hline Note. DD &
\end{tabular}

Note. $\mathrm{DD}=$ developmental delay of unknown etiology. 
Table 8

Correlations Between Adaptive Functioning Raw Scores at Each Time Point

\begin{tabular}{|c|c|c|c|c|c|c|c|c|c|c|c|c|c|c|c|}
\hline & \multicolumn{3}{|c|}{ Age 3} & \multicolumn{3}{|c|}{ Age 5} & \multicolumn{3}{|c|}{ Age 8} & \multicolumn{3}{|c|}{ Age 10} & \multicolumn{3}{|c|}{ Age 15} \\
\hline & SOC & $\mathrm{COM}$ & DL & SOC & $\mathrm{COM}$ & DL & $\mathrm{SOC}$ & $\mathrm{COM}$ & DL & $\mathrm{SOC}$ & $\mathrm{COM}$ & DL & $\mathrm{SOC}$ & $\mathrm{COM}$ & DL \\
\hline Age 3 & & & & & & & & & & & & & & & \\
\hline Socialization & --- & $.80 * *$ & $.81 * *$ & $.64 * *$ & $.63 * *$ & $.55 * *$ & $.56 * *$ & $.50 * *$ & $.57 * *$ & $.54 * *$ & $.56^{* *}$ & $.56 * *$ & $.54 * *$ & $.55 * *$ & $.58 * *$ \\
\hline Communication & $.80 * *$ & --- & $.80 * *$ & $.63 * *$ & $.72 * *$ & $.57 * *$ & $.58 * *$ & $.57 * *$ & $.60 * *$ & $.58 * *$ & $.60 * *$ & $.61 * *$ & $.52 * *$ & $.55 * *$ & $.56^{* *}$ \\
\hline $\begin{array}{r}\text { Daily living } \\
\text { Age } 5\end{array}$ & $.81 * *$ & $.80 * *$ & --- & $.64 * *$ & $.67 * *$ & $.64 * *$ & $.53 * *$ & $.51 * *$ & $.57 * *$ & $.56 * *$ & $.57 * *$ & $.60 * *$ & $.53 * *$ & $.57 * *$ & $.59 * *$ \\
\hline Socialization & $.64 * *$ & $.63 * *$ & $.64 * *$ & --- & $.84 * *$ & $.87 * *$ & $.70 * *$ & $.68 * *$ & $.73 * *$ & $.69 * *$ & $.70 * *$ & $.68 * *$ & $.67 * *$ & $.67 * *$ & $.70 * *$ \\
\hline Communication & $.63 * *$ & $.72 * *$ & $.67 * *$ & $.84 * *$ & --- & $.85 * *$ & $.74 * *$ & $.79 * *$ & $.81 * *$ & $.70 * *$ & $.76^{* *}$ & $.74 * *$ & $.72 * *$ & $.76^{* *}$ & $.76^{* *}$ \\
\hline $\begin{array}{c}\text { Daily living } \\
\text { Age } 8\end{array}$ & $.55 * *$ & $.57 * *$ & $.64 * *$ & $.87 * *$ & $.85^{* *}$ & --- & $.66^{* *}$ & $.69 * *$ & $.73 * *$ & $.66^{* *}$ & $.70 * *$ & $.69 * *$ & $.68 * *$ & $.69 * *$ & $.73 * *$ \\
\hline Socialization & $.56 * *$ & $.58 * *$ & $.53 * *$ & $.70 * *$ & $.74 * *$ & $.66^{* *}$ & --- & $.83 * *$ & $.89 * *$ & $.73 * *$ & $.72 * *$ & $.73 * *$ & $.71 * *$ & $.69 * *$ & $.72 * *$ \\
\hline Communication & $.50 * *$ & $.57 * *$ & $.51 * *$ & $.68 * *$ & $.79 * *$ & $.69 * *$ & $.83 * *$ & --- & $.90 * *$ & $.70 * *$ & $.78 * *$ & $.75 * *$ & $.71 * *$ & $.73 * *$ & $.73 * *$ \\
\hline $\begin{array}{l}\text { Daily living } \\
\text { Age } 10\end{array}$ & $.57 * *$ & $.60 * *$ & $.57 * *$ & $.73 * *$ & $.81 * *$ & $.73 * *$ & $.89 * *$ & $.90 * *$ & --- & $.73 * *$ & $.78 * *$ & $.79 * *$ & $.71 * *$ & $.74 * *$ & $.76^{* *}$ \\
\hline Socialization & $.54 * *$ & $.58 * *$ & $.56 * *$ & $.69 * *$ & $.70 * *$ & $.66^{* *}$ & $.73 * *$ & $.70 * *$ & $.73 * *$ & --- & $.91 * *$ & $.92 * *$ & $.70 * *$ & $.72 * *$ & $.73 * *$ \\
\hline Communication & $.56 * *$ & $.60 * *$ & $.57 * *$ & $.70 * *$ & $.76 * *$ & $.70 * *$ & $.72 * *$ & $.78 * *$ & $.78 * *$ & $.91 * *$ & --- & $.94 * *$ & $.74 * *$ & $.81 * *$ & $.80 * *$ \\
\hline $\begin{array}{l}\text { Daily living } \\
\text { Age } 15\end{array}$ & $.56 * *$ & $.61 * *$ & $.60 * *$ & $.68^{* *}$ & $.74 * *$ & $.69 * *$ & $.73 * *$ & $.75^{* *}$ & $.79 * *$ & $.92 * *$ & $.94 * *$ & --- & $.72 * *$ & $.78 * *$ & $.79 * *$ \\
\hline Socialization & $.54 * *$ & $.52 * *$ & $.53 * *$ & $.67 * *$ & $.72 * *$ & $.68 * *$ & $.71 * *$ & $.71 * *$ & $.71 * *$ & $.70 * *$ & $.74 * *$ & $.72 *$ & --- & $.91 * *$ & $.91 * *$ \\
\hline Communication & $.55 * *$ & $.55 * *$ & $.57 * *$ & $.67 * *$ & $.76 * *$ & $.69 * *$ & $.69 * *$ & $.73 * *$ & $.74 * *$ & $.72 * *$ & $.81 * *$ & $.78 * *$ & $.91 * *$ & --- & $.95 * *$ \\
\hline Daily living & $.58 * *$ & $.56 * *$ & $.59 * *$ & $.70 * *$ & $.76 * *$ & $.73 * *$ & $.72 * *$ & $.73 * *$ & $.76 * *$ & $.73 * *$ & $.80 * *$ & $.79 * *$ & $.91 * *$ & $.95 * *$ & --- \\
\hline
\end{tabular}

Note. ${ }^{*} \mathrm{p}<0.05, * * \mathrm{p}<0.01$. SOC $=$ Socialization, $\mathrm{COM}=$ Communication, $\mathrm{DL}=$ Daily Living Skills. 
Table 9

Correlations Between Covariates and Adaptive Functioning Outcomes

\begin{tabular}{|c|c|c|c|c|c|c|c|c|c|c|c|c|c|c|c|}
\hline & & \multicolumn{5}{|c|}{ Family and child demographic information } & \multicolumn{5}{|c|}{ Classroom externalizing behaviors } & \multicolumn{4}{|c|}{ Classroom placement type } \\
\hline & & $\begin{array}{l}\text { Child } \\
\text { gender }\end{array}$ & $\begin{array}{c}\text { Family } \\
\text { SES }\end{array}$ & $\mathrm{DD}$ & MI & $\mathrm{DS}$ & Age 3 & Age 5 & Age 8 & $\begin{array}{c}\text { Age } \\
10 \\
\end{array}$ & $\begin{array}{c}\text { Age } \\
15 \\
\end{array}$ & Age 5 & Age 8 & $\begin{array}{c}\text { Age } \\
10 \\
\end{array}$ & Age 15 \\
\hline \multicolumn{16}{|l|}{ Age 3} \\
\hline $\mathrm{SOC}$ & -0.14 & -0.02 & $0.20 * *$ & -0.12 & -0.08 & $-0.22 * *$ & -0.1 & $-0.17 *$ & $-0.18^{*}$ & -0.06 & $-0.20 *$ & -0.16 & $-0.26 * *$ & $-0.25 * *$ & $-0.27 * *$ \\
\hline $\mathrm{COM}$ & -0.10 & -0.07 & $0.23 * *$ & 0.02 & $-0.25 * *$ & $-0.29 * *$ & $-0.20 * *$ & $-0.17 *$ & $-0.20 * *$ & -0.12 & -0.17 & $-0.17 *$ & $-0.31 * *$ & $-0.32 * *$ & $-0.31 * *$ \\
\hline $\mathrm{DL}$ & -0.06 & -0.06 & $0.31 * *$ & -0.10 & $-0.20 * *$ & $-0.21 * *$ & -0.14 & $-0.17 *$ & $-0.16^{*}$ & -0.04 & $-0.23 * *$ & -0.16 & $-0.24 *$ & $-0.24 *$ & $-0.31 * *$ \\
\hline Total & -0.10 & -0.06 & $0.27 * *$ & -0.06 & $-0.20 * *$ & $-0.26 * *$ & $-0.16^{*}$ & $-0.18^{*}$ & $-0.19 *$ & -.08 & $-0.21 *$ & -0.18 & $-0.29 * *$ & $-0.30 * *$ & $-0.32 * *$ \\
\hline \multicolumn{16}{|l|}{ Age 5} \\
\hline SOC & -0.01 & -0.12 & $0.20 * *$ & -0.01 & $-0.19^{*}$ & -0.12 & $-0.30 * *$ & -0.11 & $-0.20 * *$ & -0.13 & -0.18 & $-0.25 * *$ & $-0.30 * *$ & $-0.35 * *$ & $-0.33 * *$ \\
\hline $\mathrm{COM}$ & -0.02 & -0.07 & $0.22 * *$ & 0.04 & $-0.27 * *$ & -0.12 & $-0.24 * *$ & -0.09 & $-0.19 *$ & -0.14 & -0.19 & $-0.24 * *$ & $-0.37 * *$ & $-0.37 * *$ & $-0.43 * *$ \\
\hline $\mathrm{DL}$ & -0.01 & -0.10 & $0.22 * *$ & -0.02 & $-0.21 * *$ & -0.09 & $-0.19^{*}$ & -0.06 & -0.14 & -0.11 & -0.17 & $-0.27 * *$ & $-0.30 * *$ & $-0.35^{* *}$ & $-0.39 * *$ \\
\hline Total & -0.02 & -0.10 & $0.23 * *$ & $<0.01$ & $-0.23 * *$ & $-0-11$ & $-0.25 * *$ & -0.09 & $-0.18^{*}$ & -0.13 & -0.19 & $-0.27 * *$ & $-0.34 * *$ & $-0.38 * *$ & $-0.41 * *$ \\
\hline \multicolumn{16}{|l|}{ Age 8} \\
\hline SOC & -0.09 & -0.14 & 0.14 & 0.06 & $-0.21 * *$ & -0.14 & $-0.25 * *$ & $-0.27 * *$ & $-0.31 * *$ & $-0.17 *$ & $-0.19 *$ & $-0.20 *$ & $-0.35 * *$ & $-0.35 * *$ & $-0.40 * *$ \\
\hline $\mathrm{COM}$ & -0.03 & -0.13 & $0.19^{*}$ & 0.05 & $-0.24 * *$ & -0.09 & $-0.18^{*}$ & -0.11 & $-0.23 * *$ & $-0.17 *$ & $-0.19 *$ & $-0.20 * *$ & $-0.36 * *$ & $-0.38 * *$ & $-0.39 * *$ \\
\hline $\mathrm{DL}$ & -0.06 & -0.14 & $0.25 * *$ & -0.02 & $-0.24 * *$ & -0.08 & $-0.17^{*}$ & $-0.19 *$ & $-0.27 * *$ & -0.13 & -0.15 & $-0.21 * *$ & $-0.37 * *$ & $-0.36 * *$ & $-0.40 * *$ \\
\hline Total & -0.06 & -0.14 & $0.21 * *$ & 0.02 & $-0.24 * *$ & -0.10 & $-0.20 * *$ & $-0.19^{*}$ & $-0.28 * *$ & -0.16 & $-0.18^{*}$ & $-0.21 * *$ & $-0.38 * *$ & $-0.38 * *$ & $-0.41 * *$ \\
\hline \multicolumn{16}{|l|}{ Age 10} \\
\hline SOC & -0.05 & -0.12 & $0.15^{*}$ & 0.04 & $-0.20 * *$ & -0.06 & $-0.19^{*}$ & $-0.18^{*}$ & $-0.28 * *$ & -0.14 & -0.15 & $-0.22 *$ & $-0.35 * *$ & $-0.39 * *$ & $-0.36 * *$ \\
\hline $\mathrm{COM}$ & -0.04 & -0.12 & 0.17 & 0.01 & $-0.18^{*}$ & -0.04 & $-0.15^{*}$ & -0.13 & $-0.21 * *$ & -0.16 & $-0.16^{*}$ & $-0.24 * *$ & $-0.38 * *$ & $-0.43 * *$ & $-0.40 * *$ \\
\hline $\mathrm{DL}$ & -0.04 & -0.15 & $0.23 * *$ & -0.01 & $-0.22 * *$ & -0.04 & -0.12 & $-0.17 *$ & $-0.26 * *$ & -0.15 & -0.14 & $-0.22 * *$ & $-0.37 * *$ & $-0.42 * *$ & $-0.40 * *$ \\
\hline Total & -0.05 & -0.13 & $0.20 * *$ & $<0.01$ & $-0.21 * *$ & -0.04 & $-0.15^{*}$ & $-0.16^{*}$ & $-0.25 * *$ & -0.16 & $-0.15^{*}$ & $-0.23 * *$ & $-0.37 * *$ & $-0.43 * *$ & $-0.40 * *$ \\
\hline \multicolumn{16}{|l|}{ Age 15} \\
\hline SOC & -0.04 & -0.01 & -0.02 & -0.06 & 0.04 & -0.05 & $-0.15^{*}$ & -0.05 & -0.14 & -0.12 & $-0.21 * *$ & $-0.23 * *$ & $-0.30 * *$ & $-0.32 * *$ & $-0.39 * *$ \\
\hline $\mathrm{COM}$ & -0.05 & -0.08 & 0.08 & -0.08 & 0.01 & -0.04 & -0.12 & -0.03 & -0.12 & -0.12 & $-0.20 * *$ & $-0.27 * *$ & $-0.31 * *$ & $-0.35 * *$ & $-0.43 * *$ \\
\hline $\mathrm{DL}$ & -0.02 & -0.08 & 0.13 & -0.10 & -0.02 & -0.04 & -0.12 & -0.06 & $-0.15^{*}$ & -0.14 & $-0.19 *$ & $-0.26 * *$ & $-0.32 * *$ & $-0.37 * *$ & $-0.43 * *$ \\
\hline Total & -0.04 & -0.07 & 0.09 & -0.09 & $<0.01$ & -0.04 & -0.13 & -0.05 & -0.14 & -0.13 & $-0.21 * *$ & $-0.26 * *$ & $-0.32 * *$ & $-0.36 * *$ & $-0.43 * *$ \\
\hline
\end{tabular}

Note. ${ }^{*} \mathrm{p}<0.05, * * \mathrm{p}<0.01$. SOC $=$ socialization, $\mathrm{COM}=$ communication, $\mathrm{DL}=$ daily living skills. For child's gender, $1=$ male, $0=$ female. For each

disability category, $1=$ diagnosis within that category, $0=$ other diagnosis. For classroom placement type, $1=$ substantially separate setting, $0=$

inclusive setting. $\mathrm{DD}=$ developmental delay of unknown etiology; $\mathrm{MI}=$ motor impairment. 
Table 10

Correlations Between Predictors and Adaptive Functioning Outcomes

\begin{tabular}{|c|c|c|c|c|}
\hline & $\begin{array}{l}\text { Home learning } \\
\text { environment }\end{array}$ & $\begin{array}{l}\text { Child evocative } \\
\text { effects }\end{array}$ & $\begin{array}{l}\text { Mother-child } \\
\text { interaction }\end{array}$ & $\begin{array}{c}\text { Negative life } \\
\text { events }\end{array}$ \\
\hline \multicolumn{5}{|c|}{ Adaptive functioning } \\
\hline \multicolumn{5}{|l|}{ Age 3} \\
\hline Socialization & $0.18^{*}$ & -0.15 & $0.15^{*}$ & $0.17 *$ \\
\hline Communication & $0.20^{*}$ & -0.09 & $0.16^{*}$ & 0.12 \\
\hline Daily living & 0.13 & -0.14 & $0.20 * *$ & 0.11 \\
\hline Total & $0.18^{*}$ & -0.13 & $0.18^{*}$ & 0.14 \\
\hline \multicolumn{5}{|l|}{ Age 5} \\
\hline Socialization & 0.12 & -0.09 & $0.22 * *$ & $0.16^{*}$ \\
\hline Communication & 0.12 & -0.09 & $0.22 * *$ & 0.13 \\
\hline Daily living & 0.08 & -0.11 & $0.19 *$ & 0.13 \\
\hline Total & 0.11 & -0.10 & $0.22 * *$ & 0.14 \\
\hline \multicolumn{5}{|l|}{ Age 8} \\
\hline Socialization & 0.11 & 0.04 & $0.20^{*}$ & 0.14 \\
\hline Communication & 0.09 & -0.01 & $0.16^{*}$ & $0.18^{*}$ \\
\hline Daily living & 0.08 & -0.01 & $0.18^{*}$ & $0.17 *$ \\
\hline Total & 0.09 & $<0.01$ & $0.18^{*}$ & $0.17 *$ \\
\hline \multicolumn{5}{|l|}{ Age 10} \\
\hline Socialization & 0.14 & -0.04 & 0.10 & $0.16^{*}$ \\
\hline Communication & 0.13 & -0.10 & 0.10 & $0.20 * *$ \\
\hline Daily living & 0.11 & -0.05 & 0.10 & $0.19 *$ \\
\hline Total & 0.13 & -0.06 & 0.10 & $0.19^{*}$ \\
\hline \multicolumn{5}{|l|}{ Age 15} \\
\hline Socialization & $0.19 *$ & $-0.18^{*}$ & $0.22 * *$ & 0.09 \\
\hline Communication & $0.17^{*}$ & -0.14 & $0.21^{* *}$ & 0.13 \\
\hline Daily living & 0.14 & -0.14 & $0.17 *$ & 0.09 \\
\hline Total & $0.17 *$ & $-0.15^{*}$ & $0.20 * *$ & 0.11 \\
\hline
\end{tabular}

Note. ${ }^{*} \mathrm{p}<0.05,{ }^{* *} \mathrm{p}<0.01$. 
Table 11

Correlations Between Predictors and Covariates

\begin{tabular}{|c|c|c|c|c|c|c|c|c|c|c|c|c|c|c|c|c|c|c|c|}
\hline Variable & 1 & 2 & 3 & 4 & 5 & 6 & 7 & 8 & 9 & 10 & 11 & 12 & 13 & 14 & 15 & 16 & 17 & 18 & 19 \\
\hline \multicolumn{20}{|c|}{ Demographic information } \\
\hline 1. Child's gender & - & $.16^{* *}$ & $.07^{*}$ & $.09^{*}$ & -.06 & -.04 & -.02 & $<0.01$ & .07 & .02 & $<.01$ & -.06 & .15 & $.22^{* *}$ & .02 & .01 & -.04 & .04 & $.09^{*}$ \\
\hline $\begin{array}{l}\text { 2. Diagnosis of Down } \\
\text { syndrome }\end{array}$ & $-.16^{* *}$ & -- & N/A & N/A & $.22^{* *}$ & -.09 & -.03 & -.03 & .07 & .10 & -.06 & .11 & $.16^{*}$ & $.26^{* *}$ & .12 & $.23^{* *}$ & .06 & $.14 * *$ & $-.09^{* *}$ \\
\hline $\begin{array}{l}\text { 3. Diagnosis of motor } \\
\text { impairment }\end{array}$ & $.07 *$ & $\mathrm{~N} / \mathrm{A}$ & -- & N/A & $\stackrel{-}{.10^{* *}}$ & -.05 & -.07 & .05 & .04 & $<0.01$ & .07 & -.11 & -.11 & -.08 & -.03 & $.14 * *$ & -.05 & $-.16^{* *}$ & .02 \\
\hline $\begin{array}{l}\text { 4. Diagnosis of other } \\
\text { DD }\end{array}$ & $.09^{*}$ & $\mathrm{~N} / \mathrm{A}$ & $\mathrm{N} / \mathrm{A}$ & - &.$\overline{1} 1^{* *}$ & .14 & .10 & -.02 & -.11 & -.10 & -.02 & $<.01$ & -.05 & $-.18^{*}$ & -.09 & $.08^{*}$ & -.01 & .03 & .07 \\
\hline $\begin{array}{l}\text { 5. Family } \\
\text { socioeconomic status }\end{array}$ & -.06 & $.22 * *$ & $-\overline{10}$ & $.11^{* *}$ & - & -.06 & .05 & .05 & .14 & .14 & -.10 & -.05 & $<0.01$ & .14 & -.09 & -.07 & $.23 * *$ & $.45 * *$ & $-.33^{* *}$ \\
\hline \multicolumn{20}{|l|}{ Classroom placement } \\
\hline 6. Age 3 & -.04 & -.09 & -.05 & .14 & -.06 & -- & $.33^{* *}$ & .16 & .13 & .13 & $<0.01$ & .09 & $<0.01$ & $<0.01$ & .04 & .13 & -.11 & .02 & .04 \\
\hline 7. Age 5 & -.02 & -.03 & -.07 & .10 & .05 & $.33^{* *}$ & - & .18 & .15 & .10 & -.04 & -.01 & -.02 & .06 &, 12 & .13 & -.04 & -.05 & -.11 \\
\hline 8. Age 8 & $<0.01$ & -.03 & .05 & -.02 & .05 & .16 & .18 & -- & $.48^{* *}$ & $.17^{* *}$ & .15 & .14 & .03 & .13 & .15 & .05 & $-.18^{*}$ & -.11 & -.09 \\
\hline 9. Age 10 & .07 & .07 & .04 & -.11 & .14 & .13 & .15 & $.48^{* *}$ & -- & $.26^{* *}$ & .22 & .14 & .11 & $.26^{* *}$ & .04 & -.02 & -.15 & $-.17^{*}$ & -.06 \\
\hline 10. Age 15 & .02 & .10 & $<0.01$ & -.10 & .14 & .13 & .10 & $.17^{* *}$ & $.26^{* *}$ & -- & .04 & .07 & .04 & .16 & .10 & .05 & -.14 & -.07 & -03 \\
\hline \multicolumn{20}{|c|}{ Classroom externalizing behaviors } \\
\hline 11. Age 3 & $<.01$ & -.06 & .07 & -.02 & -.10 & $<0.01$ & -.04 & .15 & .22 & .04 & -- & $.43^{* *}$ & $.23^{* *}$ & $.18^{*}$ & .12 & .07 & $-.33^{* *}$ & $-.33^{* *}$ & $.19^{*}$ \\
\hline 12. Age 5 & -.06 & .11 & -.11 & $<0.01$ & -.05 & .09 & -.01 & .14 & .14 & .07 & $.43^{* *}$ & -- & $.18^{*}$ & $.27^{* *}$ & $.24 * *$ & .02 & $-.24 * *$ & $-.21^{* *}$ & .11 \\
\hline 13. Age 8 & .15 & $.16^{*}$ & -.11 & -.05 & $<0.01$ & $<0.01$ & -.02 & .03 & .11 & .04 & $.23^{* *}$ & $.18^{*}$ & - & $.48^{* *}$ & .11 & .05 & $-.19^{* *}$ & -.08 & .14 \\
\hline 14. Age 10 & $.22 * *$ & $.26^{* *}$ & -.08 & $-.18^{*}$ & .14 & $<0.01$ & .06 & .13 & $.26^{* *}$ & .16 & $.18^{*}$ & $.27^{* *}$ & $.48^{* *}$ & -- & $.27 * *$ & .08 & -.14 & -.08 & .03 \\
\hline 15. Age 15 & .02 & .12 & -.03 & -.09 & -.09 & .04 & .12 & .15 & .04 & .10 & .12 & $.24 * *$ & .11 & $.27 * *$ & -- & .08 & -.05 & $-.19^{*}$ & .08 \\
\hline \multicolumn{20}{|c|}{$\begin{array}{l}\text { Early childhood home and family } \\
\text { environment indicators }\end{array}$} \\
\hline $\begin{array}{l}\text { 16. Child evocative } \\
\text { effects }\end{array}$ & .01 & $.23 * *$ & $.14 * *$ & $.08^{*}$ & -.07 & .13 & .13 & .05 & -.02 & .05 & .07 & .02 & .05 & .08 & .08 & -- & $-.11^{* *}$ & $-.09 *$ & $.17^{* *}$ \\
\hline $\begin{array}{l}\text { 17. Mother-child } \\
\text { interaction }\end{array}$ & -.04 & .06 & -.05 & -.01 & $.23^{* *}$ & -.11 & -.04 & $-.18^{*}$ & -.15 & -.14 & $.33^{* * *}$ & $.24 * *$ & $-.19^{*}$ & -.14 & -.05 & $.11^{-} *$ & -- & $.37 * *$ & $-.14 * *$ \\
\hline $\begin{array}{l}\text { 18. Home } \\
\text { environment }\end{array}$ & .04 & $.14 * *$ & $-\overline{-16}^{* *}$ & .03 & $.45^{* *}$ & .02 & -.05 & -.11 & $-.18^{*}$ & -.07 & 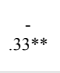 & $.21^{* *}$ & -.08 & -.08 & $-.19^{*}$ & $-.09^{*}$ & $.37 * *$ & -- & $-.19^{* *}$ \\
\hline $\begin{array}{l}\text { 19. Negative life } \\
\text { events }\end{array}$ & $.09 *$ & $\begin{array}{r}- \\
.09 * *\end{array}$ & .02 & .07 & $.33^{* *}$ & .04 & -.11 & -.09 & -.06 & -.03 & $.19 *$ & .11 & .14 & .03 & .08 & $.17 * *$ & $-.14 * *$ & $-.19 * *$ & -- \\
\hline
\end{tabular}

Note. ${ }^{*} \mathrm{p}<0.05,{ }^{*} \mathrm{p}<0.01$. For child's gender, $1=$ male, $0=$ female. For the 3 disability categories, $1=$ diagnosis within that category, $0=$ other diagnosis. $\mathrm{DD}=$ developmental delay of unknown etiology. For classroom placement type, 1 = substantially separate setting, $0=$ inclusive setting. 
Table 12

Multilevel Models Predicting Socialization

\begin{tabular}{|c|c|c|c|c|c|c|}
\hline & \multicolumn{2}{|c|}{$\begin{array}{c}\text { Model } 1 \\
\text { (unconditional model) }\end{array}$} & \multicolumn{2}{|c|}{$\begin{array}{l}\text { Model } 2 \text { (including } \\
\text { covariates) }\end{array}$} & \multicolumn{2}{|c|}{$\begin{array}{c}\text { Model } 3 \text { (including } \\
\text { covariates and } \\
\text { predictors) }\end{array}$} \\
\hline & Estimate & $\begin{array}{l}\text { Standard } \\
\text { error }\end{array}$ & Estimate & $\begin{array}{l}\text { Standard } \\
\text { error }\end{array}$ & Estimate & $\begin{array}{l}\text { Standard } \\
\text { error }\end{array}$ \\
\hline \multicolumn{7}{|l|}{ Fixed effects } \\
\hline Intercept & $22.24 * *$ & 1.06 & $20.88 * *$ & 1.90 & $21.01 * *$ & 1.85 \\
\hline Age & $0.40 * *$ & 0.03 & $0.39 * *$ & 0.01 & $0.39 * *$ & 0.01 \\
\hline $\mathrm{Age}^{2}$ & 0.00004 & $<0.01$ & -- & -- & -- & -- \\
\hline \multicolumn{7}{|l|}{$\begin{array}{l}\text { Predictors of the } \\
\text { intercept }\end{array}$} \\
\hline Diagnosis of DD & & & $8.82 * *$ & 2.41 & $8.32 * *$ & 2.34 \\
\hline Diagnosis of MI & & & 3.94 & 2.29 & 3.78 & 2.22 \\
\hline $\begin{array}{l}\text { Substantially } \\
\text { separate setting }\end{array}$ & & & $-4.81 * *$ & 1.35 & $-4.68 * *$ & 1.36 \\
\hline $\begin{array}{l}\text { Externalizing } \\
\text { behaviors }\end{array}$ & & & $-3.46 * *$ & 0.58 & $-3.54 * *$ & 0.59 \\
\hline Negative life events & & & & & $0.65 * *$ & 0.20 \\
\hline $\begin{array}{l}\text { Mother-child } \\
\text { interaction }\end{array}$ & & & & & 0.27 & 0.16 \\
\hline \multicolumn{7}{|l|}{$\begin{array}{l}\text { Predictors of the } \\
\text { slope }\end{array}$} \\
\hline $\begin{array}{l}\text { Mother-child } \\
\text { interaction }\end{array}$ & & & & & $0.004^{*}$ & 0.002 \\
\hline \multicolumn{7}{|l|}{$\begin{array}{l}\text { Covariance } \\
\text { parameters }\end{array}$} \\
\hline Residual $\left(\sigma_{\epsilon}^{2}\right)$ & $131.69^{* *}$ & 8.20 & $118.93 * *$ & 7.34 & $118.65^{* *}$ & 7.29 \\
\hline $\begin{array}{l}\text { Variability in initial } \\
\text { status }\left(\sigma_{0}^{2}\right)\end{array}$ & $95.00 * *$ & 17.93 & $105.21 * *$ & 17.52 & $94.71 * *$ & 16.38 \\
\hline $\begin{array}{l}\text { Variability in slope } \\
\left(\sigma^{2}{ }_{1}\right)\end{array}$ & $0.01 * *$ & $<0.01$ & $0.02 * *$ & 0.003 & $0.02 * *$ & $<0.01$ \\
\hline \multicolumn{7}{|l|}{ Fit Statistics } \\
\hline -2 Log Likelihood & \multicolumn{2}{|c|}{7298.50} & \multicolumn{2}{|c|}{7124.32} & \multicolumn{2}{|c|}{7107.52} \\
\hline AIC & \multicolumn{2}{|c|}{7312.50} & \multicolumn{2}{|c|}{7142.32} & \multicolumn{2}{|c|}{7131.52} \\
\hline $\mathrm{BIC}$ & \multicolumn{2}{|c|}{7346.04} & \multicolumn{2}{|c|}{7142.52} & \multicolumn{2}{|c|}{7188.75} \\
\hline
\end{tabular}

Note. ${ }^{*} \mathrm{p}<0.05, * * \mathrm{p}<0.01$. Child externalizing behaviors and placement in a substantially separate setting are level-1 predictors; all other predictors are level-2. DD = developmental delay of unknown etiology; $\mathrm{MI}=$ motor impairment. 
Table 13

Multilevel Models Predicting Communication

\begin{tabular}{|c|c|c|c|c|c|c|}
\hline & \multicolumn{2}{|c|}{$\begin{array}{c}\text { Model } 1 \\
\text { (unconditional model) }\end{array}$} & \multicolumn{2}{|c|}{$\begin{array}{l}\text { Model } 2 \text { (including } \\
\text { covariates) }\end{array}$} & \multicolumn{2}{|c|}{$\begin{array}{c}\text { Model } 3 \text { (including } \\
\text { covariates and } \\
\text { predictors) }\end{array}$} \\
\hline & Estimate & $\begin{array}{l}\text { Standard } \\
\text { error }\end{array}$ & Estimate & $\begin{array}{l}\text { Standard } \\
\text { error }\end{array}$ & Estimate & $\begin{array}{l}\text { Standard } \\
\text { error }\end{array}$ \\
\hline \multicolumn{7}{|l|}{ Fixed effects } \\
\hline Intercept & $24.60 * *$ & 1.44 & $21.65 * *$ & 2.77 & $21.69 * *$ & 2.71 \\
\hline Age & $0.71 * *$ & 0.03 & $0.56 * *$ & 0.06 & $0.56 * *$ & 0.06 \\
\hline $\mathrm{Age}^{2}$ & $-0.002 * *$ & $<0.01$ & -0.0004 & $<0.01$ & -0.0004 & 0.0004 \\
\hline \multicolumn{7}{|l|}{$\begin{array}{l}\text { Predictors of the } \\
\text { intercept }\end{array}$} \\
\hline Diagnosis of DD & & & $12.32 * *$ & 3.68 & $11.90 * *$ & 3.61 \\
\hline Diagnosis of MI & & & 6.75 & 3.47 & $6.75 *$ & 3.39 \\
\hline $\begin{array}{l}\text { Substantially } \\
\text { separate setting }\end{array}$ & & & $-5.24 * *$ & 1.54 & $-5.13 * *$ & 1.56 \\
\hline $\begin{array}{l}\text { Externalizing } \\
\text { behaviors }\end{array}$ & & & $-2.21 * *$ & 0.72 & $-2.13 * *$ & 0.73 \\
\hline Negative life events & & & & & $0.73 *$ & 0.28 \\
\hline $\begin{array}{l}\text { Mother-child } \\
\text { interaction }\end{array}$ & & & & & $0.59 * *$ & 0.22 \\
\hline \multicolumn{7}{|l|}{$\begin{array}{l}\text { Predictors of the } \\
\text { slope }\end{array}$} \\
\hline DD*Age interaction & & & $0.20 *$ & 0.08 & $0.20 *$ & 0.08 \\
\hline $\begin{array}{l}\mathrm{DD}^{*} \mathrm{Age}^{2} \\
\text { interaction }\end{array}$ & & & $-0.002 * *$ & $<0.01$ & $-0.002 * *$ & $<0.01$ \\
\hline MI*Age interaction & & & $0.17 *$ & 0.08 & $0.17 *$ & $<0.01$ \\
\hline $\begin{array}{l}\text { MI* Age }^{2} \\
\text { interaction }\end{array}$ & & & $-0.002 * *$ & $<0.01$ & $-0.002 * *$ & $<0.01$ \\
\hline \multicolumn{7}{|l|}{$\begin{array}{l}\text { Covariance } \\
\text { parameters }\end{array}$} \\
\hline Residual $\left(\sigma_{\epsilon}^{2}\right)$ & $176.53 * *$ & 11.64 & $161.07 * *$ & 10.59 & $161.19 * *$ & 10.61 \\
\hline $\begin{array}{l}\text { Variability in initial } \\
\text { status }\left(\sigma_{0}^{2}\right)\end{array}$ & $225.51 * *$ & 34.31 & $225.57 * *$ & 32.74 & $206.47 * *$ & 30.66 \\
\hline $\begin{array}{l}\text { Variability in slope } \\
\left(\sigma_{1}^{2}\right)\end{array}$ & $0.02 * *$ & $<0.01$ & $0.03 * *$ & $<0.01$ & $0.03 * *$ & $<0.01$ \\
\hline \multicolumn{7}{|l|}{ Fit Statistics } \\
\hline -2 Log Likelihood & \multicolumn{2}{|c|}{7667.10} & \multicolumn{2}{|c|}{7462.99} & \multicolumn{2}{|c|}{7451.13} \\
\hline AIC & \multicolumn{2}{|c|}{7681.10} & \multicolumn{2}{|c|}{7490.99} & \multicolumn{2}{|c|}{7483.13} \\
\hline $\mathrm{BIC}$ & \multicolumn{2}{|c|}{7714.64} & \multicolumn{2}{|c|}{7557.75} & \multicolumn{2}{|c|}{7559.43} \\
\hline
\end{tabular}

Note. ${ }^{*} \mathrm{p}<0.05, * * \mathrm{p}<0.01$. Child externalizing behaviors and placement in a substantially separate setting are level-1 predictors; all other predictors are level-2. DD = developmental delay of unknown etiology; $\mathrm{MI}=$ motor impairment. 
Table 14

Multilevel Models Predicting Daily Living Skills

\begin{tabular}{|c|c|c|c|c|c|c|}
\hline & \multicolumn{2}{|c|}{$\begin{array}{c}\text { Model } 1 \\
\text { (unconditional model) }\end{array}$} & \multicolumn{2}{|c|}{$\begin{array}{l}\text { Model } 2 \text { (including } \\
\text { covariates) }\end{array}$} & \multicolumn{2}{|c|}{$\begin{array}{c}\text { Model } 3 \text { (including } \\
\text { covariates and } \\
\text { predictors) }\end{array}$} \\
\hline & Estimate & $\begin{array}{l}\text { Standard } \\
\text { error }\end{array}$ & Estimate & $\begin{array}{l}\text { Standard } \\
\text { error }\end{array}$ & Estimate & $\begin{array}{c}\text { Standarc } \\
\text { error }\end{array}$ \\
\hline \multicolumn{7}{|l|}{ Fixed effects } \\
\hline Intercept & $22.61 * *$ & 1.85 & $24.24 * *$ & 3.26 & $24.30 * *$ & 3.19 \\
\hline Age & $1.29 * *$ & 0.05 & $0.98 * *$ & 0.08 & $0.98 * *$ & 0.08 \\
\hline $\mathrm{Age}^{2}$ & $-0.01 * *$ & $<0.01$ & $-0.003^{* *}$ & $<0.01$ & $-0.002 * *$ & $<0.01$ \\
\hline \multicolumn{7}{|l|}{$\begin{array}{l}\text { Predictors of the } \\
\text { intercept }\end{array}$} \\
\hline Diagnosis of DD & & & $10.84 *$ & 4.33 & $10.26^{*}$ & 4.23 \\
\hline Diagnosis of MI & & & 2.47 & 4.09 & 2.29 & 3.99 \\
\hline $\begin{array}{l}\text { Substantially } \\
\text { separate setting }\end{array}$ & & & $-8.86^{* *}$ & 1.87 & $-8.65 * *$ & 1.88 \\
\hline $\begin{array}{l}\text { Externalizing } \\
\text { behaviors }\end{array}$ & & & $-3.69 * *$ & 0.94 & $-3.56^{* *}$ & 0.95 \\
\hline Negative life events & & & & & $0.90 * *$ & 0.33 \\
\hline $\begin{array}{l}\text { Mother-child } \\
\text { interaction }\end{array}$ & & & & & $0.64 *$ & 0.25 \\
\hline \multicolumn{7}{|l|}{$\begin{array}{l}\text { Predictors of the } \\
\text { slope }\end{array}$} \\
\hline DD*Age interaction & & & $0.47 * *$ & 0.11 & $0.47 * *$ & 0.11 \\
\hline $\begin{array}{l}\mathrm{DD}^{*} \mathrm{Age}^{2} \\
\text { interaction }\end{array}$ & & & $-0.003 * *$ & $<0.01$ & $-0.003 * *$ & $<0.01$ \\
\hline MI*Age interaction & & & $0.32 * *$ & 0.11 & $0.32 * *$ & 0.11 \\
\hline $\begin{array}{l}\mathrm{MI}^{*} \mathrm{Age}^{2} \\
\text { interaction }\end{array}$ & & & $-0.003 * *$ & $<0.01$ & $-0.003 * *$ & $<0.01$ \\
\hline \multicolumn{7}{|l|}{$\begin{array}{l}\text { Covariance } \\
\text { parameters }\end{array}$} \\
\hline Residual $\left(\sigma_{\epsilon}^{2}\right)$ & $329.57 * *$ & 19.15 & $308.36^{* *}$ & 18.11 & $307.95^{* *}$ & 18.07 \\
\hline $\begin{array}{l}\text { Variability in initial } \\
\text { status }\left(\sigma_{0}^{2}\right)\end{array}$ & $349.03 * *$ & 54.13 & $246.47 * *$ & 43.15 & $220.15^{* *}$ & 40.34 \\
\hline $\begin{array}{l}\text { Variability in slope } \\
\left(\sigma^{2}{ }_{1}\right)\end{array}$ & $0.05 * *$ & 0.01 & $0.05 * *$ & 0.01 & $0.05 * *$ & 0.01 \\
\hline \multicolumn{7}{|l|}{ Fit Statistics } \\
\hline -2 Log Likelihood & \multicolumn{2}{|c|}{8226.80} & \multicolumn{2}{|c|}{7961.84} & \multicolumn{2}{|c|}{7950.60} \\
\hline $\mathrm{AIC}$ & \multicolumn{2}{|c|}{8238.80} & \multicolumn{2}{|c|}{7989.84} & \multicolumn{2}{|c|}{7982.60} \\
\hline $\mathrm{BIC}$ & \multicolumn{2}{|c|}{8267.54} & \multicolumn{2}{|c|}{8056.60} & \multicolumn{2}{|c|}{8058.90} \\
\hline
\end{tabular}


Table 15

Multilevel Models Predicting Total Scores of Adaptive Functioning

\begin{tabular}{|c|c|c|c|c|c|c|}
\hline & \multicolumn{2}{|c|}{$\begin{array}{l}\text { Model } 1 \text { (unconditional } \\
\text { model) }\end{array}$} & \multicolumn{2}{|c|}{$\begin{array}{l}\text { Model } 2 \text { (including } \\
\text { covariates) }\end{array}$} & \multicolumn{2}{|c|}{$\begin{array}{c}\text { Model } 3 \text { (including } \\
\text { covariates and predictors) }\end{array}$} \\
\hline & Estimate & $\begin{array}{c}\text { Standard } \\
\text { error }\end{array}$ & Estimate & $\begin{array}{c}\text { Standard } \\
\text { error }\end{array}$ & Estimate & $\begin{array}{c}\text { Standard } \\
\text { error }\end{array}$ \\
\hline \multicolumn{7}{|l|}{ Fixed effects } \\
\hline Intercept & $69.45 * *$ & 3.91 & $69.09 * *$ & 7.58 & $69.34 * *$ & 7.40 \\
\hline Age & $2.40 * *$ & 0.10 & $1.76^{* *}$ & 0.17 & $1.76^{* *}$ & 0.17 \\
\hline $\mathrm{Age}^{2}$ & $-0.007 * *$ & $<0.01$ & -0.002 & $<0.01$ & -0.002 & $<0.01$ \\
\hline \multicolumn{7}{|l|}{$\begin{array}{l}\text { Predictors of the } \\
\text { intercept }\end{array}$} \\
\hline Diagnosis of DD & & & $28.65 * *$ & 10.07 & $27.23 * *$ & 9.82 \\
\hline Diagnosis of MI & & & 9.20 & 9.48 & 9.00 & 9.21 \\
\hline $\begin{array}{l}\text { Substantially } \\
\text { separate setting }\end{array}$ & & & $-18.07 * *$ & 4.30 & $-17.72 * *$ & 4.34 \\
\hline $\begin{array}{l}\text { Externalizing } \\
\text { behaviors }\end{array}$ & & & $-8.92 * *$ & 2.01 & $-8.71 * *$ & 2.02 \\
\hline Negative life events & & & & & $2.25 * *$ & 0.77 \\
\hline $\begin{array}{l}\text { Mother-child } \\
\text { interaction }\end{array}$ & & & & & $1.58 * *$ & 0.59 \\
\hline \multicolumn{7}{|l|}{$\begin{array}{l}\text { Predictors of the } \\
\text { slope }\end{array}$} \\
\hline DD*Age interaction & & & $0.86 * *$ & 0.24 & $0.87 * *$ & 0.24 \\
\hline $\begin{array}{l}\mathrm{DD}^{*} \mathrm{Age}^{2} \\
\text { interaction }\end{array}$ & & & $-0.007 * *$ & $<0.01$ & $-0.007 * *$ & $<0.01$ \\
\hline MI*Age interaction & & & $0.72 * *$ & 0.22 & $0.72 * *$ & 0.22 \\
\hline $\begin{array}{l}\mathrm{MI}^{*} \mathrm{Age}^{2} \\
\text { interaction }\end{array}$ & & & $-0.006^{* *}$ & $<0.01$ & $-0.006^{* *}$ & $<0.01$ \\
\hline \multicolumn{7}{|l|}{$\begin{array}{l}\text { Covariance } \\
\text { parameters }\end{array}$} \\
\hline Residual $\left(\sigma_{\epsilon}^{2}\right)$ & $1514.89 * *$ & 94.70 & $1317.11 * *$ & 81.59 & $\begin{array}{c}1316.16^{*} \\
*\end{array}$ & 81.52 \\
\hline $\begin{array}{l}\text { Variability in initial } \\
\text { status }\left(\sigma_{0}^{2}\right)\end{array}$ & $1517.91 * *$ & 249.28 & $1602.73 * *$ & 243.69 & $\begin{array}{c}1440.06^{*} \\
* \\
\end{array}$ & 226.16 \\
\hline $\begin{array}{l}\text { Variability in slope } \\
\left(\sigma_{1}^{2}\right)\end{array}$ & $0.22 * *$ & 0.04 & $0.26^{* *}$ & 0.04 & $0.26^{* *}$ & 0.04 \\
\hline \multicolumn{7}{|l|}{ Fit Statistics } \\
\hline -2 Log Likelihood & \multicolumn{2}{|c|}{9530.42} & \multicolumn{2}{|c|}{9296.15} & \multicolumn{2}{|c|}{9283.45} \\
\hline AIC & \multicolumn{2}{|c|}{9544.42} & \multicolumn{2}{|c|}{9324.15} & \multicolumn{2}{|c|}{9315.45} \\
\hline $\mathrm{BIC}$ & \multicolumn{2}{|c|}{9577.96} & \multicolumn{2}{|c|}{9390.91} & \multicolumn{2}{|c|}{9391.74} \\
\hline
\end{tabular}

Note. ${ }^{*} \mathrm{p}<0.05, * * \mathrm{p}<0.01$. Child externalizing behaviors and placement in a substantially separate setting are level-1 predictors; all other predictors are level-2. DD = developmental delay of unknown etiology; $\mathrm{MI}=$ motor impairment. 
Table 16

Negative life events experienced by families

\begin{tabular}{lc}
\hline \multicolumn{1}{c}{ Event } & Number of families (\%) \\
\hline Decreased income & $36(21.1)$ \\
Went into debt & $31(18.3)$ \\
Death in family & $25(14.7)$ \\
Friends death & $24(14.1)$ \\
Legal problems & $17(10.0)$ \\
Marital separation & $16(9.4)$ \\
Alcohol-drug problem & $13(7.6)$ \\
Divorce & $12(7.1)$ \\
Trouble at work & $11(6.5)$ \\
Trouble at school & $9(5.3)$ \\
\hline Note. The numbers in this table reflect the number of families that experienced each \\
event at least once during the early childhood period. The only events that families \\
experienced more than once were going into debt (experienced twice by four families), \\
decrease in income (experienced twice by five families), and legal problems (experienced \\
twice by one family).
\end{tabular}


Table 17

Predicted values of adaptive functioning raw scores for children with Down syndrome (based on indicators of the early childhood home and family environment) and corresponding age equivalents

\begin{tabular}{|c|c|c|c|c|}
\hline & $\begin{array}{l}\text { High quality } \\
\text { mother-child } \\
\text { interaction }\end{array}$ & $\begin{array}{l}\text { Low quality } \\
\text { mother-child } \\
\text { interaction }\end{array}$ & $\begin{array}{l}\text { High number of } \\
\text { negative life } \\
\text { events }\end{array}$ & $\begin{array}{l}\text { Low number of } \\
\text { negative life } \\
\text { events }\end{array}$ \\
\hline \multicolumn{5}{|c|}{ Socialization } \\
\hline Age 3 & 21.01 (1 yr. 8 mo.) & 21.01 (1 yr. 8 mo.) & 24.15 (1 yr. $10 \mathrm{mo})$. & 17.87 (1 yr. 5 mo.) \\
\hline Age 5 & 21.84 (1 yr. 8 mo.) & 21.74 (1 yr. 8 mo.) & 24.93 (1 yr. $11 \mathrm{mo})$. & 18.65 (1 yr. 6 mo. $)$ \\
\hline Age 8 & 23.32 (1 yr. 9 mo.) & 23.08 (1 yr. 9 mo.) & 26.10 (2 yr. 0 mo. $)$ & 19.82 (1 yr. 7 mo.) \\
\hline Age 10 & 23.91 (1 yr. 10 mo.) & 23.57 (1 yr. 10 mo.) & 26.88 (2 yr. 1 mo.) & 20.60 (1 yr. 8 mo. $)$ \\
\hline Age 15 & 25.98 ( 2 yr. 0 mo. $)$ & 25.40 (1 yr. 11 mo.) & 28.83 (2 yr. 2 mo.) & 22.55 (1 yr. 9 mo. $)$ \\
\hline \multicolumn{5}{|c|}{ Communication } \\
\hline Age 3 & 25.20 (1 yr. 6 mo.) & 18.18 (1 yr. 2 mo.) & 25.22 (1 yr. 6 mo.) & 18.16 (1 yr. 2 mo.) \\
\hline Age 5 & 26.32 (1 yr. 6 mo.) & 19.30 (1 yr. 3 mo.) & 26.42 (1 yr. 6 mo.) & 19.36 (1 yr. 3 mo.) \\
\hline Age 8 & 28.01 (1 yr. 7 mo. $)$ & 20.98 (1 yr. 4 mo.) & 28.02 (1 yr. 7 mo. $)$ & 20.96 (1 yr. 4 mo.) \\
\hline Age 10 & 29.12 (1 yr. 8 mo.) & 22.10 (1 yr. 4 mo.) & 29.14 (1 yr. 8 mo.) & 22.08 (1 yr. 4 mo.) \\
\hline Age 15 & 31.92 (1 yr. 9 mo.) & 24.90 (1 yr. 6 mo.) & 31.94 (1 yr. 9 mo.) & 24.88 (1 yr. 6 mo.) \\
\hline \multicolumn{5}{|c|}{$\begin{array}{l}\text { Daily living } \\
\text { skills }\end{array}$} \\
\hline Age 3 & 28.11 (1 yr. 10 mo.) & 20.49 (1 yr. 6 mo.) & 28.65 (1 yr. 11 mo.) & 19.95 (1 yr. 6 mo.) \\
\hline Age 5 & 30.00 (1 yr. $11 \mathrm{mo})$. & 22.38 (1 yr. 8 mo.) & 30.54 (2 yr. 0 mo.) & 21.85 (1 yr. 7 mo.) \\
\hline Age 8 & 32.90 (2 yr. 1 mo. $)$ & 25.28 (yr. 9 mo.) & 33.44 ( 2 yr. 1 mo.) & 24.74 (1 yr. 9 mo.) \\
\hline Age 10 & 34.81 ( 2 yr. 2 mo.) & 27.19 (1 yr. 10 mo. $)$ & 35.35 ( 2 yr. 2 mo. $)$ & 26.66 (1 yr. 10 mo. $)$ \\
\hline Age 15 & 39.52 (2 yr. 4 mo.) & 31.90 (2 yr. 0 mo.) & 40.06 ( 2 yr. 4 mo. $)$ & 31.65 (2 yr. 0 mo.) \\
\hline \multicolumn{5}{|c|}{$\begin{array}{l}\text { Total adaptive } \\
\text { functioning } \\
\text { scores }\end{array}$} \\
\hline Age 3 & 78.74 & 59.94 & 80.21 & 58.47 \\
\hline Age 5 & 82.26 & 63.46 & 83.73 & 61.99 \\
\hline Age 8 & 87.54 & 68.74 & 89.01 & 67.27 \\
\hline Age 10 & 91.06 & 72.26 & 92.53 & 70.79 \\
\hline Age 15 & 99.86 & 81.06 & 101.33 & 79.59 \\
\hline
\end{tabular}

Note. Age equivalent scores are not available for total adaptive functioning scores.

High quality mother-child interaction represents mother-child interaction scores one standard deviation above the sample mean. Low quality mother-child interaction represents scores one standard deviation below the sample mean. High number of negative life events represents a number of negative life events equal to one standard deviation more than the sample mean; low number of negative life events represents a number of negative life events equal to one standard deviation less than the sample mean. 
Table 18

Predicted values of adaptive functioning raw scores for children with motor impairment (based on indicators of the early childhood home and family environment) and corresponding age equivalents

\begin{tabular}{|c|c|c|c|c|}
\hline & $\begin{array}{l}\text { High quality } \\
\text { mother-child } \\
\text { interaction }\end{array}$ & $\begin{array}{l}\text { Low quality } \\
\text { mother-child } \\
\text { interaction }\end{array}$ & $\begin{array}{l}\text { High number of } \\
\text { negative life } \\
\text { events }\end{array}$ & $\begin{array}{l}\text { Low number of } \\
\text { negative life } \\
\text { events }\end{array}$ \\
\hline \multicolumn{5}{|c|}{ Socialization } \\
\hline Age 3 & 21.01 (1 yr. 8 mo.) & 21.01 (1 yr. 8 mo.) & 24.15 (1 yr. $10 \mathrm{mo})$. & 17.87 (1 yr. 5 mo.) \\
\hline Age 5 & 21.84 (1 yr. 8 mo.) & 21.74 ( 1 yr. 8 mo.) & 24.93 (1 yr. $11 \mathrm{mo})$. & 18.65 (1 yr. 6 mo. $)$ \\
\hline Age 8 & 23.32 (1 yr. 9 mo. $)$ & 23.08 ( 1 yr. 9 mo. $)$ & 26.10 (2 yr. 0 mo. $)$ & 19.82 (1 yr. 7 mo.) \\
\hline Age 10 & 23.91 (1 yr. 10 mo. $)$ & 23.57 (1 yr. 10 mo. $)$ & 26.88 (2 yr. 1 mo.) & 20.60 (1 yr. 8 mo. $)$ \\
\hline Age 15 & 25.98 ( 2 yr. 0 mo. $)$ & 25.40 (1 yr. $11 \mathrm{mo})$. & 28.83 (2 yr. 2 mo.) & 22.55 (1 yr. 9 mo. $)$ \\
\hline \multicolumn{5}{|c|}{ Communication } \\
\hline Age 3 & 25.20 (1 yr. 6 mo.) & 18.17 (1 yr. 2 mo.) & 31.97 (1 yr. 9 mo.) & 24.91 (1 yr. 6 mo.) \\
\hline Age 5 & 26.65 (1 yr. 7 mo.) & 19.63 (1 yr. 3 mo.) & 33.50 (1 yr. 10 mo.) & 26.45 (1 yr. 6 mo.) \\
\hline Age 8 & 28.81 (1 yr. 8 mo.) & 21.78 (1 yr. 4 mo.) & 35.57 (1 yr. $11 \mathrm{mo})$. & 28.51 (1 yr. 8 mo.) \\
\hline Age 10 & 30.21 (1 yr. 8 mo.) & 23.19 (1 yr. 5 mo.) & 36.98 (2 yr. 0 mo.) & 29.93 (1 yr. 8 mo.) \\
\hline Age 15 & 33.67 (1 yr. 10 mo.) & 26.65 (1 yr. 7 mo.) & 40.44 (2 yr. 2 mo.) & 33.39 (1 yr. 10 mo.) \\
\hline \multicolumn{5}{|c|}{$\begin{array}{l}\text { Daily living } \\
\text { skills }\end{array}$} \\
\hline Age 3 & 28.11 (1 yr. 10 mo.) & 20.49 (1 yr. 6 mo.) & 28.65 (1 yr. $11 \mathrm{mo})$. & 19.95 (1 yr. 6 mo.) \\
\hline Age 5 & 30.63 (2 yr. 0 mo.) & 23.01 (1 yr. 8 mo.) & 31.17 (2 yr. 0 mo.) & 22.47 (1 yr. 7 mo.) \\
\hline Age 8 & 34.42 (2 yr. 1 mo.) & 26.81 (1 yr. 10 mo.) & 34.96 (2 yr. 2 mo.) & 26.27 (1 yr. 10 mo.) \\
\hline Age 10 & 36.90 ( 2 yr. 3 mo.) & 29.29 (1 yr. 11 mo. $)$ & 37.74 ( 2 yr. 3 mo. $)$ & 29.04 (1 yr. 11 mo.) \\
\hline Age 15 & 42.92 (2 yr. 6 mo.) & 35.30 (2 yr. 2 mo.) & 43.47 (2 yr. 6 mo.) & 34.77 ( 2 yr. 2 mo. $)$ \\
\hline \multicolumn{5}{|c|}{$\begin{array}{l}\text { Total adaptive } \\
\text { functioning } \\
\text { scores }\end{array}$} \\
\hline Age 3 & 78.74 & 59.94 & 80.21 & 58.47 \\
\hline Age 5 & 83.68 & 64.88 & 85.14 & 63.41 \\
\hline Age 8 & 90.99 & 72.19 & 92.46 & 70.72 \\
\hline Age 10 & 95.81 & 77.01 & 97.27 & 75.54 \\
\hline Age 15 & 107.64 & 88.84 & 109.10 & 87.37 \\
\hline
\end{tabular}

Note. Age equivalent scores are not available for total adaptive functioning scores. High quality mother-child interaction represents mother-child interaction scores one standard deviation above the sample mean. Low quality mother-child interaction represents scores one standard deviation below the sample mean. High number of negative life events represents a number of negative life events equal to one standard deviation more than the sample mean; low number of negative life events represents a number of negative life events equal to one standard deviation less than the sample mean. 
Table 19

Predicted values of adaptive functioning raw scores for children with developmental delay of unknown etiology (based on indicators of the early childhood home and family environment) and corresponding age equivalents

\begin{tabular}{|c|c|c|c|c|}
\hline & $\begin{array}{l}\text { High quality } \\
\text { mother-child } \\
\text { interaction }\end{array}$ & $\begin{array}{l}\text { Low quality } \\
\text { mother-child } \\
\text { interaction }\end{array}$ & $\begin{array}{l}\text { High number of } \\
\text { negative life } \\
\text { events }\end{array}$ & $\begin{array}{l}\text { Low number of } \\
\text { negative life } \\
\text { events }\end{array}$ \\
\hline \multicolumn{5}{|c|}{ Socialization } \\
\hline Age 3 & 29.33 ( 2 yr. 2 mo.) & 29.33 ( 2 yr. 2 mo. $)$ & 32.47 (2 yr. 5 mo.) & 26.19 (2 yr. 0 mo. $)$ \\
\hline Age 5 & 30.16 ( 2 yr. 3 mo. $)$ & 30.06 ( 2 yr. 3 mo. $)$ & 33.25 ( 2 yr. 6 mo.) & 26.97 (2 yr. 1 mo. $)$ \\
\hline Age 8 & 31.40 (2 yr. 4 mo.) & 31.16 (2 yr. 4 mo.) & 34.42 (2 yr. 6 mo.) & 28.14 (2 yr. 1 mo.) \\
\hline Age 10 & 32.23 (2 yr. 5 mo.) & 31.89 ( 2 yr. 5 mo. $)$ & 35.20 (2 yr. 7 mo.) & 28.92 (2 yr. 2 mo. $)$ \\
\hline Age 15 & 34.30 (2 yr. 6 mo.) & 33.72 (2 yr. 6 mo.) & 37.15 (2 yr. 9 mo. $)$ & 30.87 (2 yr. 4 mo.) \\
\hline \multicolumn{5}{|c|}{ Communication } \\
\hline Age 3 & 37.10 (2 yr. 0 mo. $)$ & 30.08 (1 yr. 8 mo.) & 37.12 (2 yr. 0 mo.) & 30.06 (1 yr. 8 mo.) \\
\hline Age 5 & 38.61 (2 yr. 1 mo.) & 31.59 (1 yr. 9 mo.) & 38.71 (2 yr. 1 mo.) & 31.66 (1 yr. 9 mo.) \\
\hline Age 8 & 40.86 ( 2 yr. 3 mo. $)$ & 33.83 (1 yr. 10 mo.) & 40.87 ( 2 yr. 3 mo. $)$ & 33.81 (1 yr. 10 mo.) \\
\hline Age 10 & 42.32 (2 yr. 4 mo.) & 35.30 (1 yr. $11 \mathrm{mo})$. & 42.34 (2 yr. 4 mo.) & 35.29 (1 yr. 11 mo. $)$ \\
\hline Age 15 & 45.93 (2 yr. 6 mo.) & 38.91 (2 yr. 1 mo.) & 43.79 (2 yr. 5 mo.) & 36.74 ( 2 yr. 0 mo.) \\
\hline \multicolumn{5}{|c|}{$\begin{array}{l}\text { Daily living } \\
\text { skills }\end{array}$} \\
\hline Age 3 & 38.37 (2 yr. 3 mo.) & 30.75 ( 2 yr. 0 mo.) & 38.91 (2 yr. 4 mo.) & 30.21 (1 yr. 11 mo.) \\
\hline Age 5 & 41.19 (2 yr. 5 mo.) & 33.57 (2 yr. 1 mo.) & 41.73 (2 yr. 6 mo.) & 33.03 (2 yr. 1 mo.) \\
\hline Age 8 & 45.43 (2 yr. 7 mo.) & 37.82 (2 yr. 3 mo.) & 45.97 (2 yr. 8 mo.) & 37.28 ( 2 yr. 3 mo. $)$ \\
\hline Age 10 & 48.21 ( 2 yr. 9 mo. $)$ & 40.60 ( 2 yr. 5 mo. $)$ & 48.75 ( 2 yr. 10 mo. $)$ & 40.06 (2 yr. 4 mo.) \\
\hline Age 15 & 54.99 (3 yr. 1 mo.) & 47.37 ( 2 yr. 8 mo.) & 55.53 (3 yr. 2 mo.) & 46.83 ( 2 yr. 8 mo. $)$ \\
\hline \multicolumn{5}{|c|}{$\begin{array}{l}\text { Total adaptive } \\
\text { functioning } \\
\text { scores }\end{array}$} \\
\hline Age 3 & 105.97 & 87.17 & 107.44 & 85.70 \\
\hline Age 5 & 111.89 & 93.09 & 112.67 & 90.94 \\
\hline Age 8 & 119.64 & 100.83 & 120.41 & 98.68 \\
\hline Age 10 & 124.73 & 105.93 & 125.50 & 103.77 \\
\hline Age 15 & 137.21 & 118.41 & 137.99 & 116.26 \\
\hline
\end{tabular}

Note. Age equivalent scores are not available for total adaptive functioning scores. High quality mother-child interaction represents mother-child interaction scores one standard deviation above the sample mean. Low quality mother-child interaction represents scores one standard deviation below the sample mean. High number of negative life events represents a number of negative life events equal to one standard deviation more than the sample mean; low number of negative life events represents a number of negative life events equal to one standard deviation less than the sample mean. 
Figure 1. Unconditional model predicting socialization raw scores. This figure shows the trajectory of socialization raw scores.

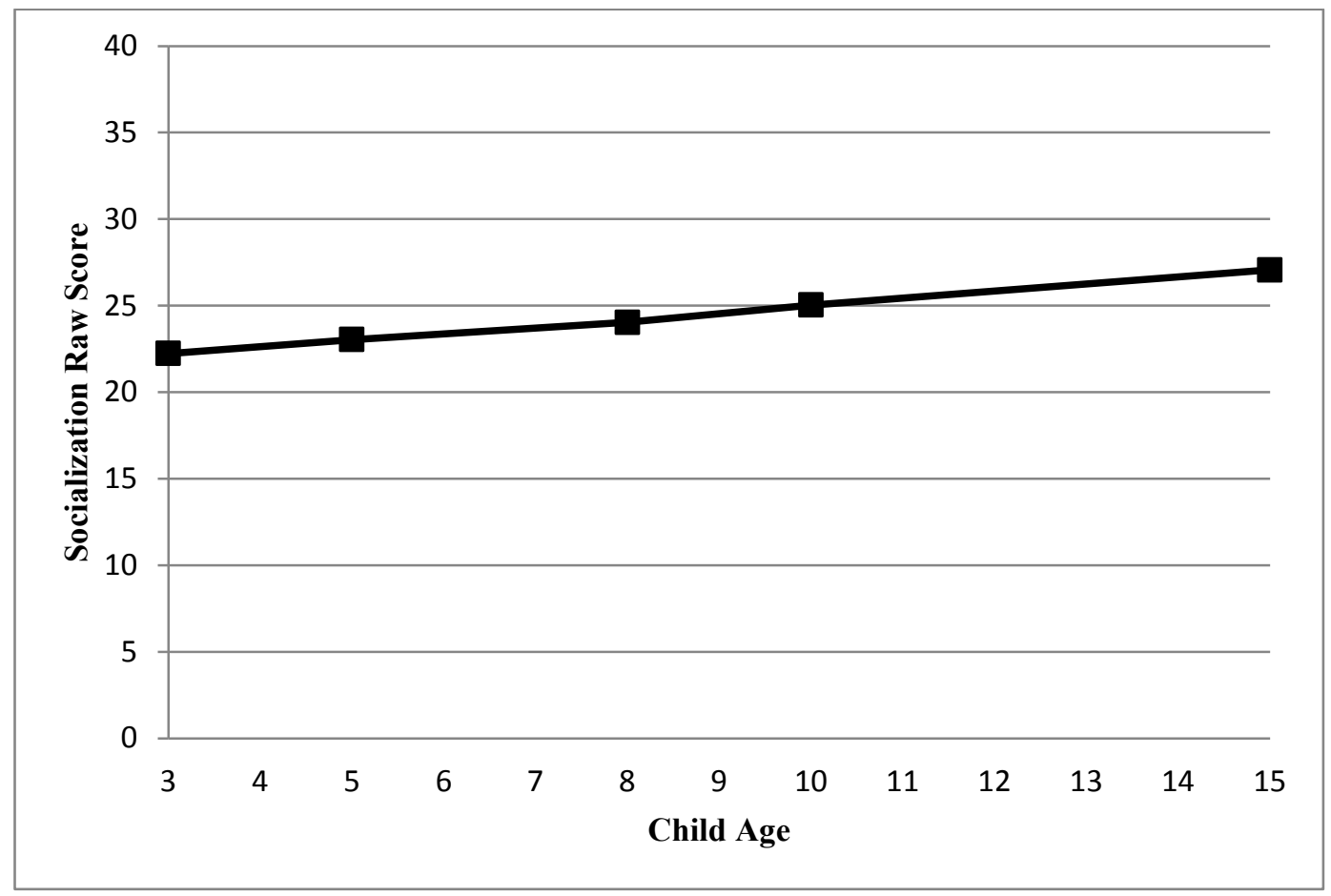

Figure 2. Unconditional model predicting communication raw scores. This figure shows the trajectory of communication raw scores.

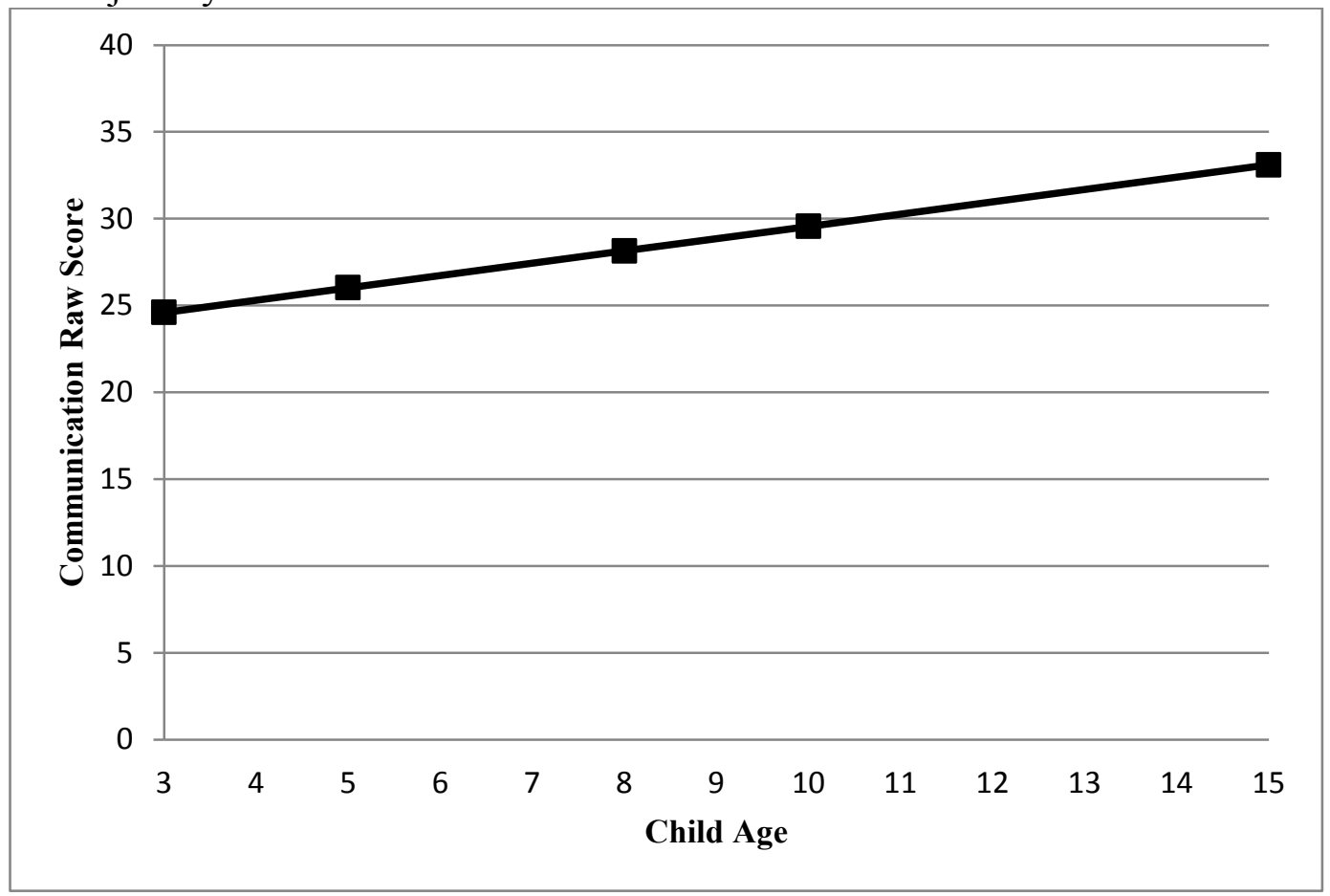


Figure 3. Unconditional model predicting daily living raw scores. This figure shows the trajectory of daily living raw scores.

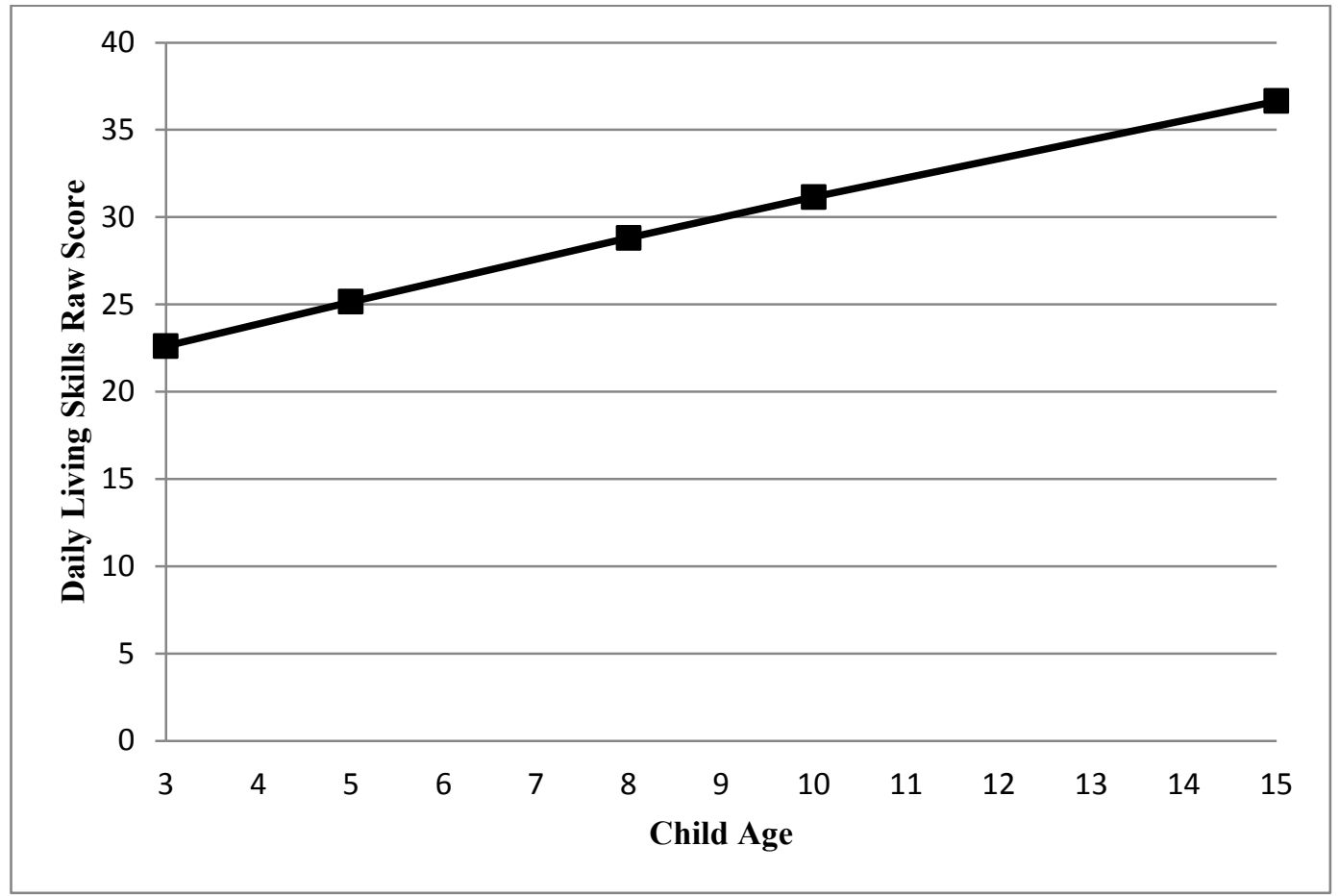

Figure 4. Unconditional model predicting total adaptive functioning raw scores. This figure shows the trajectory of total adaptive functioning raw scores.

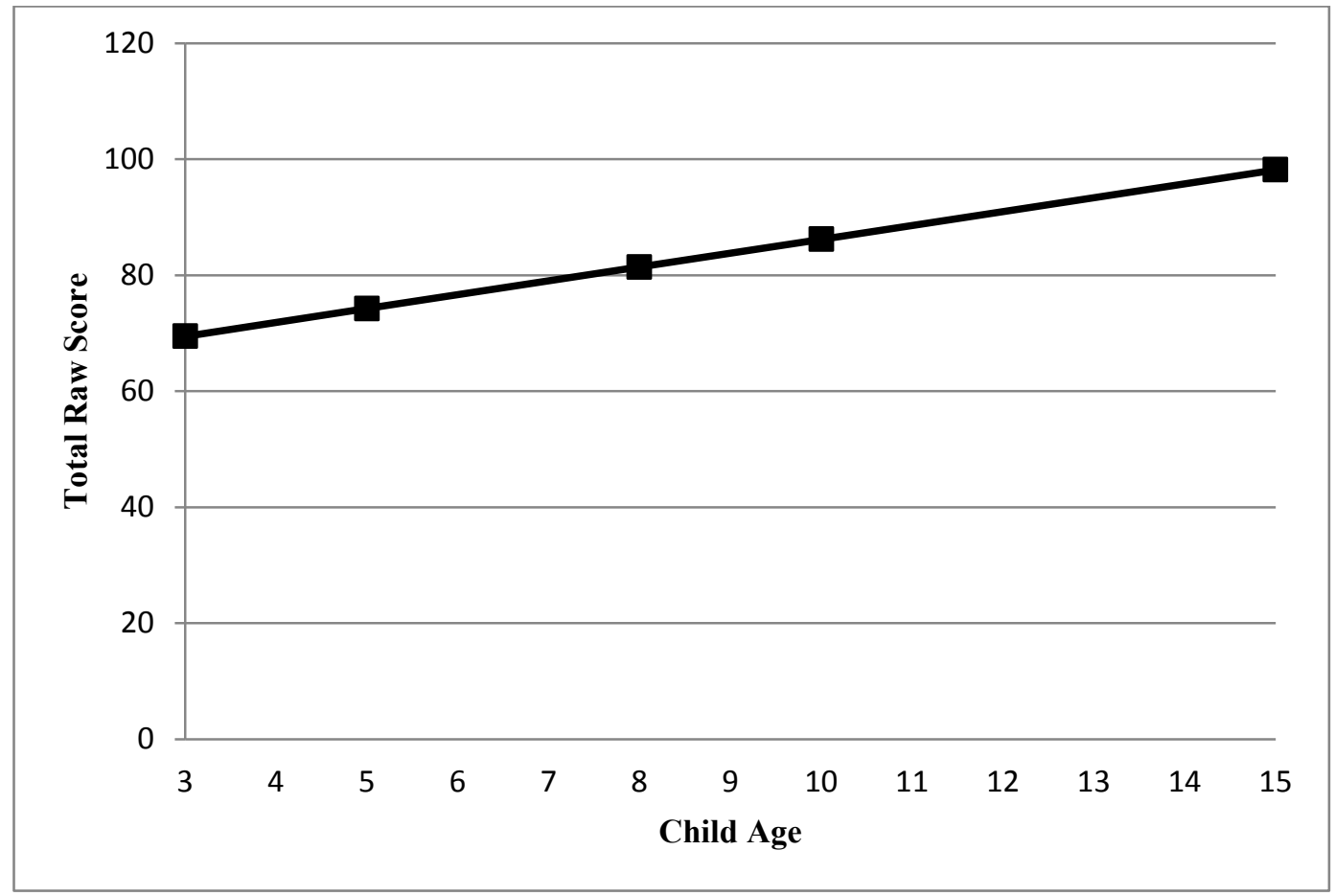


Figure 5. Mother-child interaction as a predictor of socialization scores for children with Down syndrome and motor impairment. This figure shows trajectories of socialization scores for children with Down syndrome and motor impairment, with mother-child interaction scores that are equal to one standard deviation above and below the sample mean.

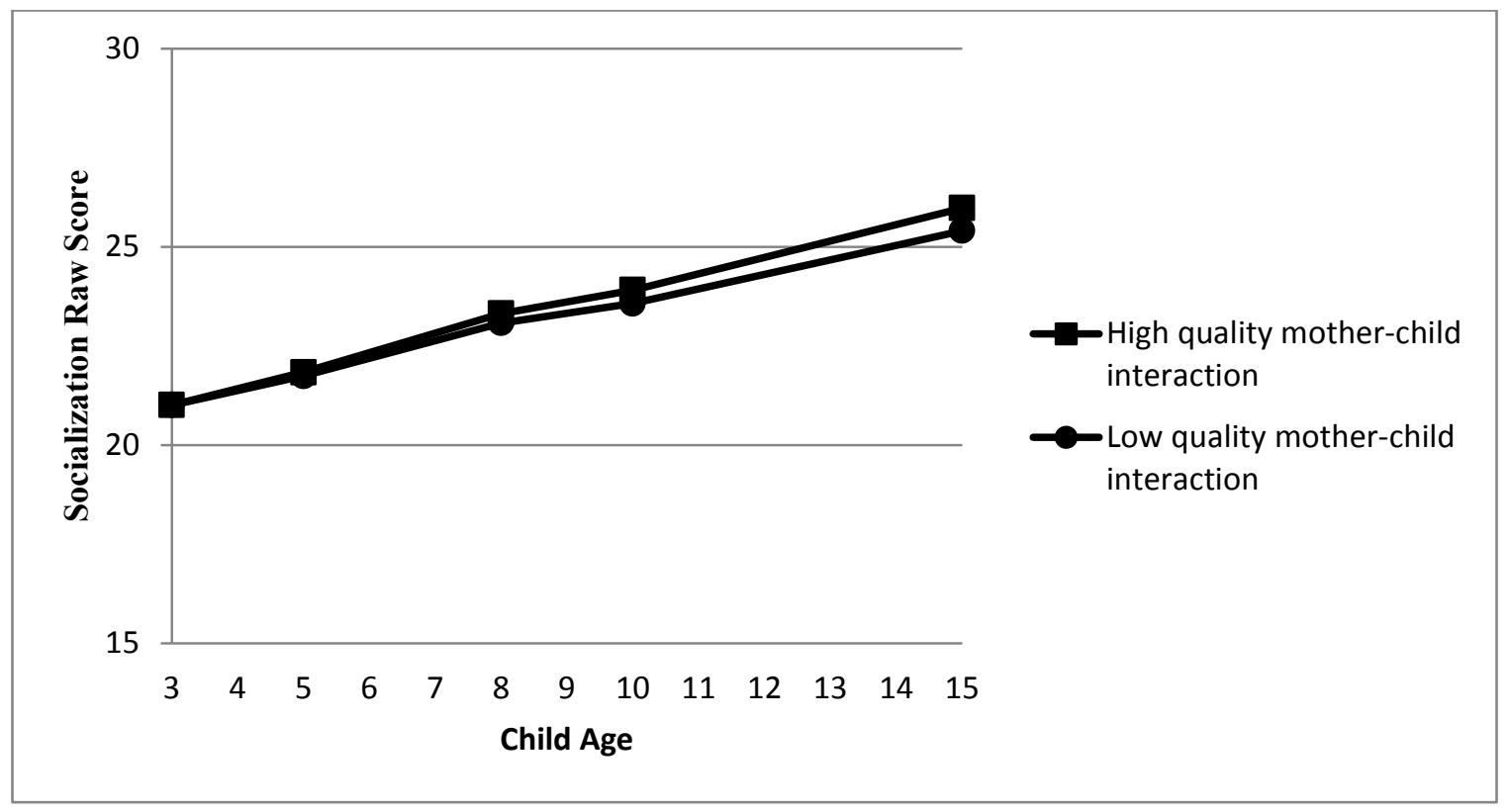

Figure 6. Mother-child interaction as a predictor of socialization scores for children with developmental delay of unknown etiology. This figure shows trajectories of socialization scores for children with developmental delay of unknown etiology with mother-child interaction scores that are equal to one standard deviation above and below the sample mean.

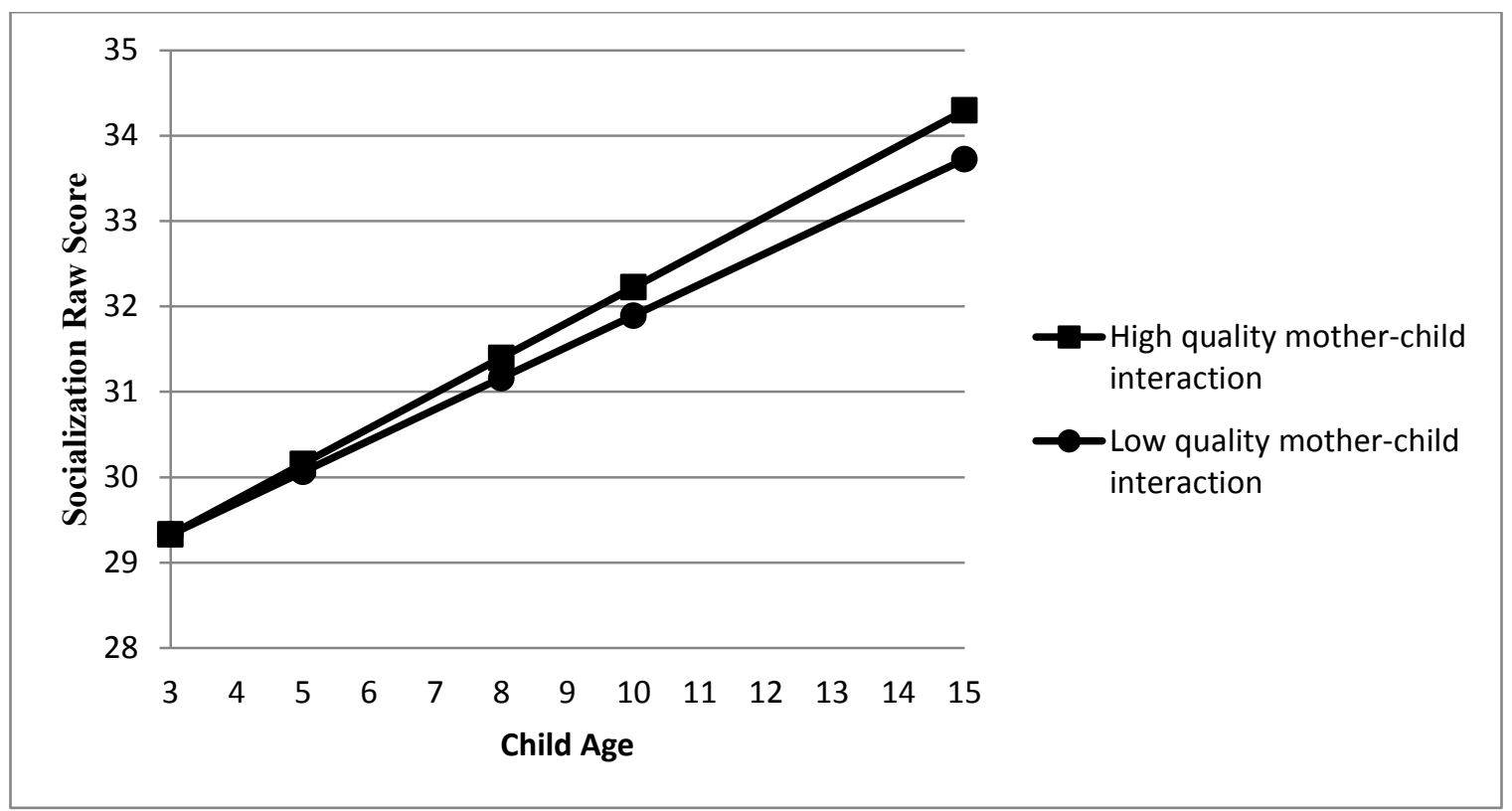


Figure 7. Negative life events as a predictor of socialization scores for children with Down syndrome and motor impairment. This figure shows trajectories of socialization scores for children with Down syndrome and motor impairment who have experienced numbers of negative life events equal to one standard deviation above and below the sample mean.

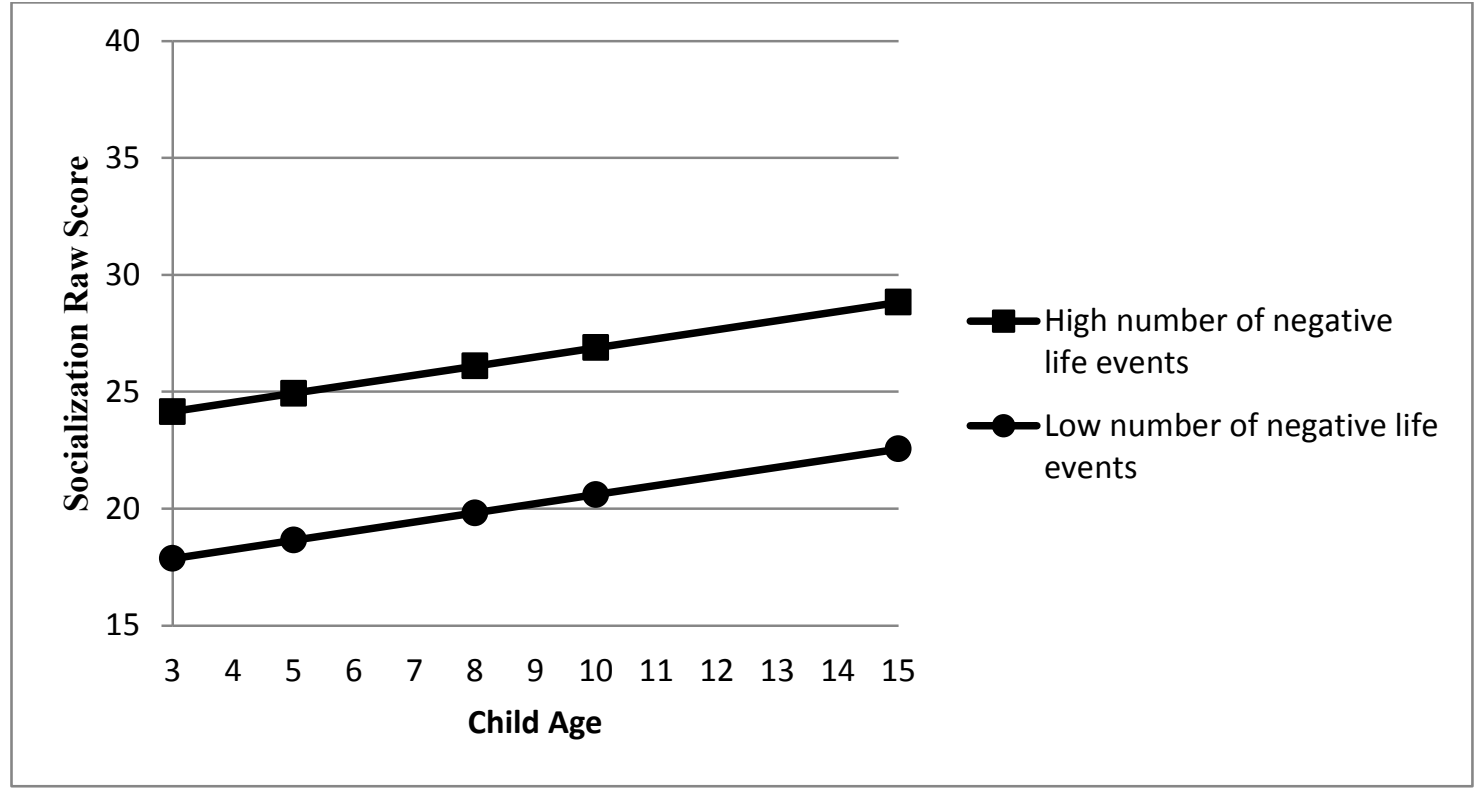

Figure 8. Negative life events as a predictor of socialization scores for children with developmental delay of unknown etiology. This figure shows trajectories of socialization scores for children with developmental delay of unknown etiology who have experienced numbers of negative life events equal to one standard deviation above and below the sample mean.

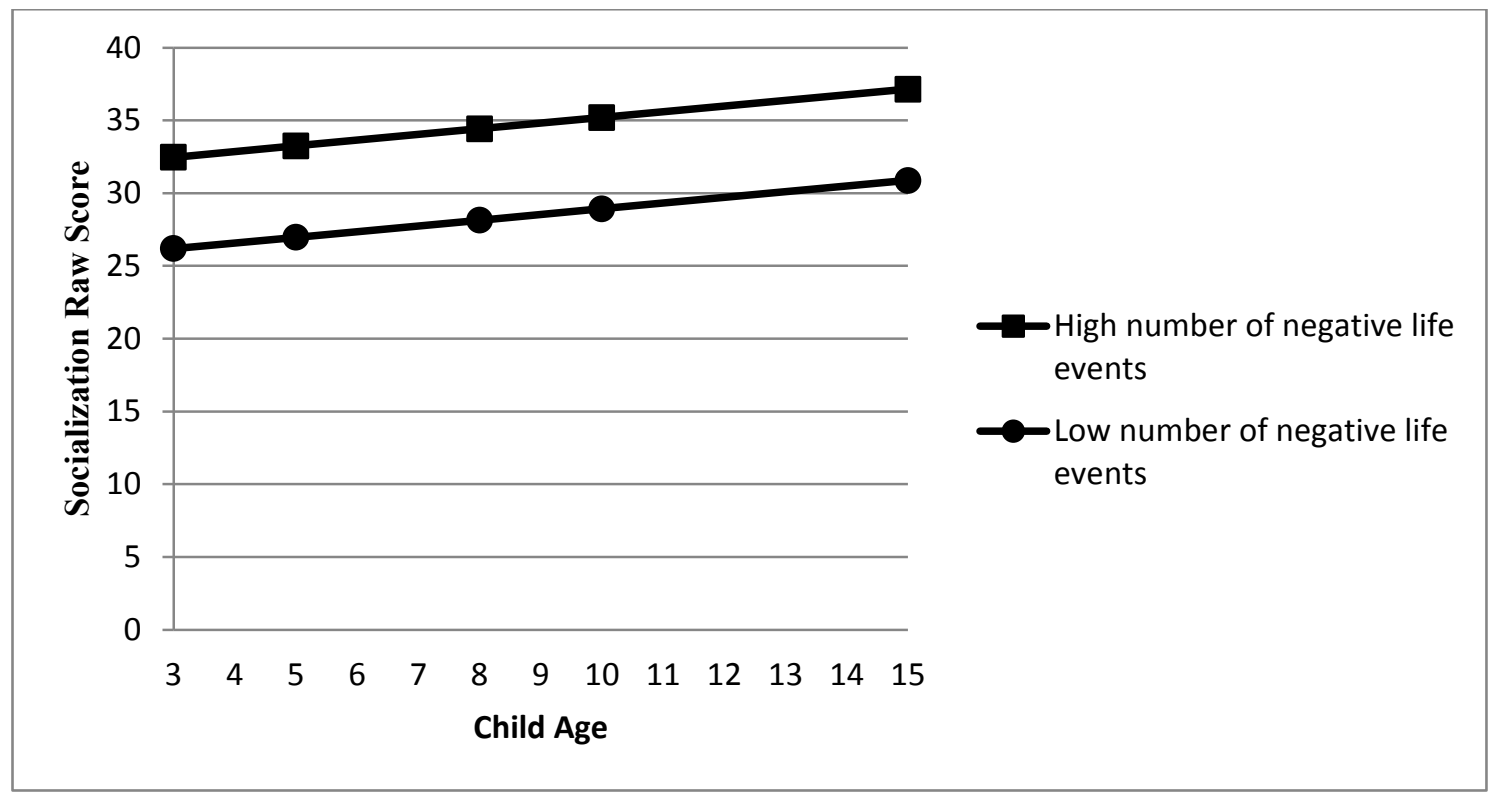


Figure 9. Mother-child interaction as a predictor of communication scores for children with Down syndrome. This figure shows trajectories of communication scores for children with Down syndrome who have mother child-interaction scores equal to one standard above and below the sample mean.

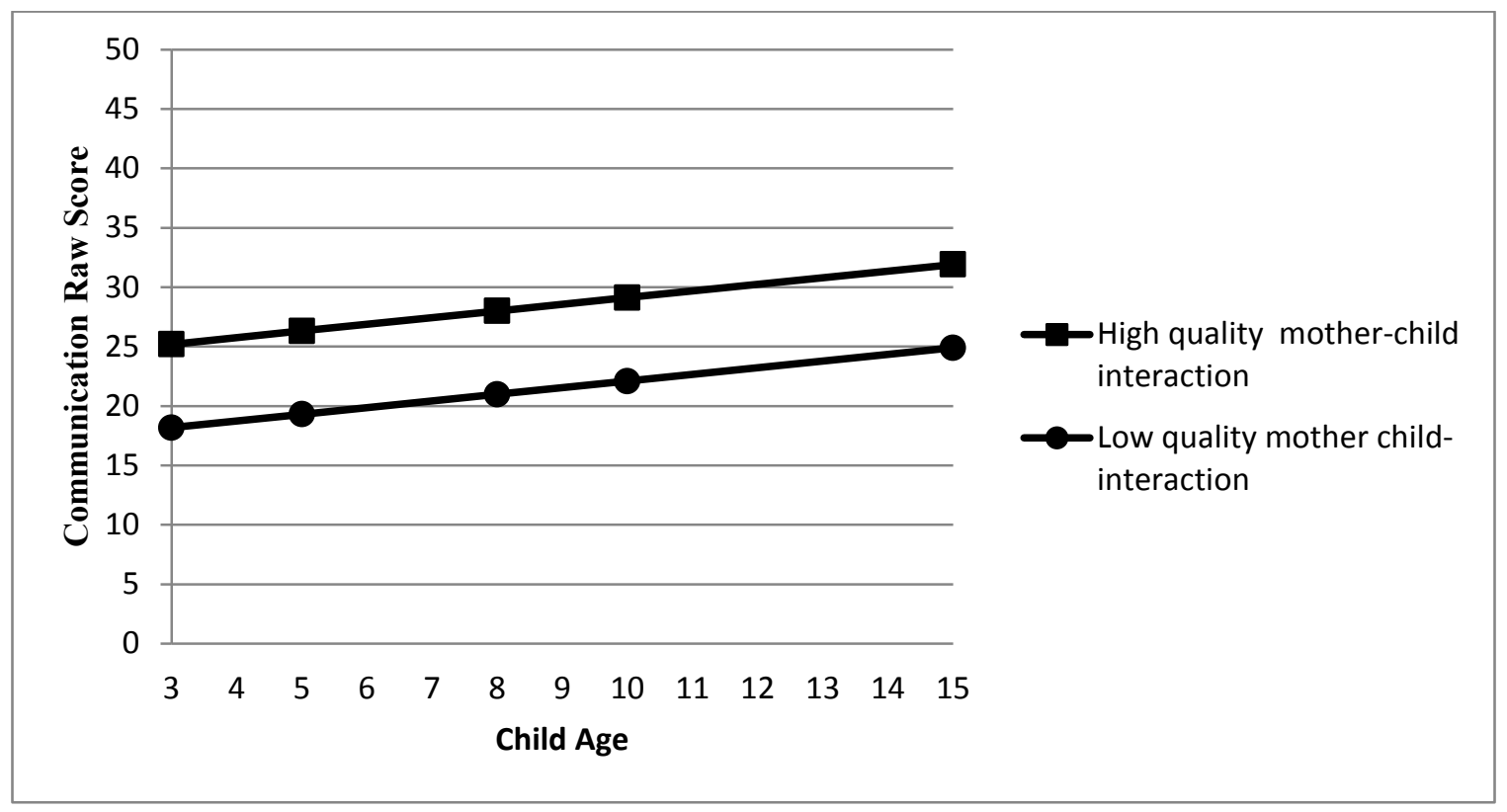

Figure 10. Mother-child interaction as a predictor of communication scores for children with motor impairment. This figure shows trajectories of communication scores for children with motor impairment who have mother child-interaction scores equal to one standard above and below the sample mean.

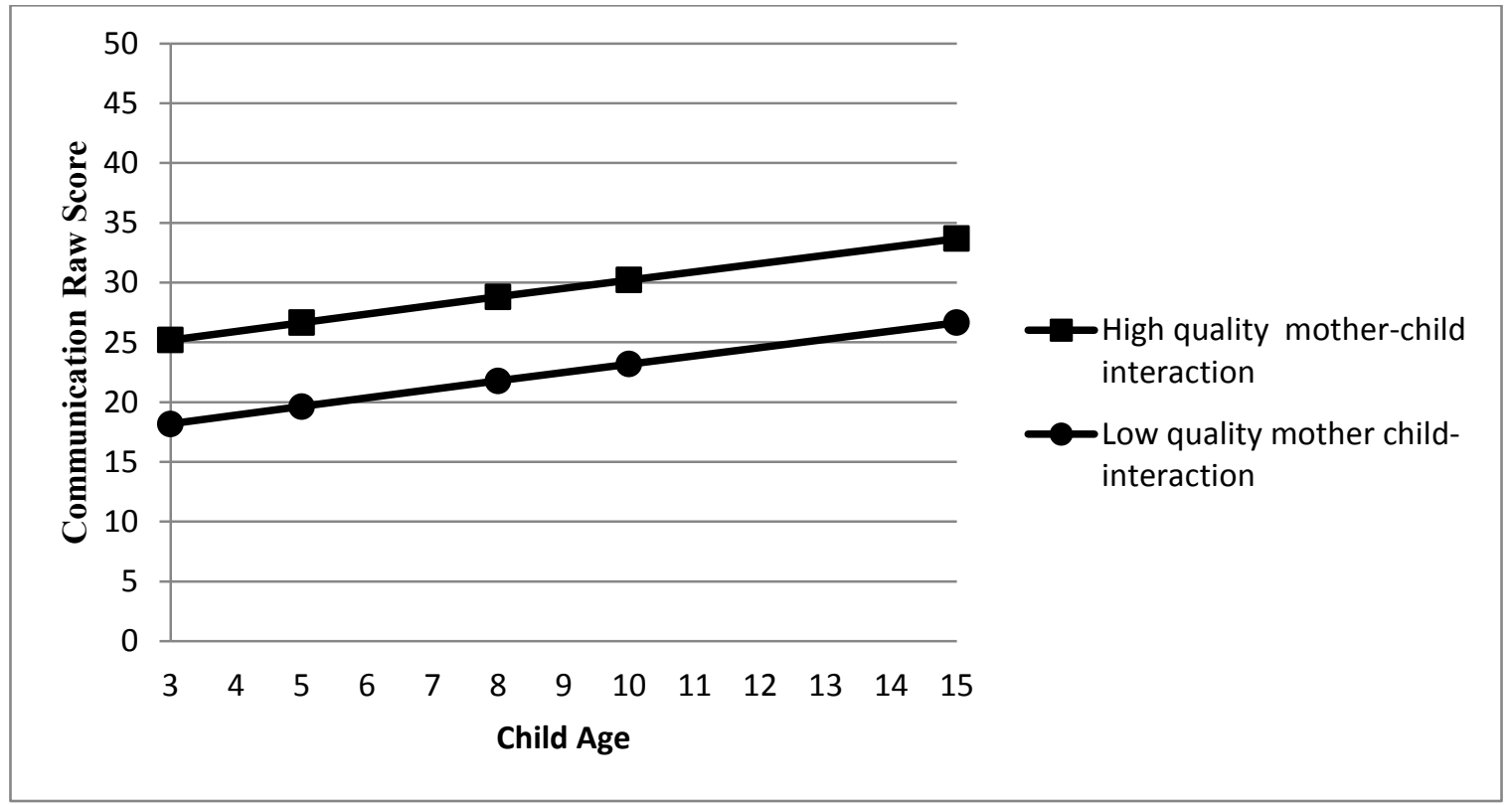


Figure 11. Mother-child interaction as a predictor of communication scores for children with developmental delay of unknown etiology. This figure shows trajectories of communication scores for children with developmental delay of unknown etiology who have mother child-interaction scores equal to one standard above and below the sample mean.

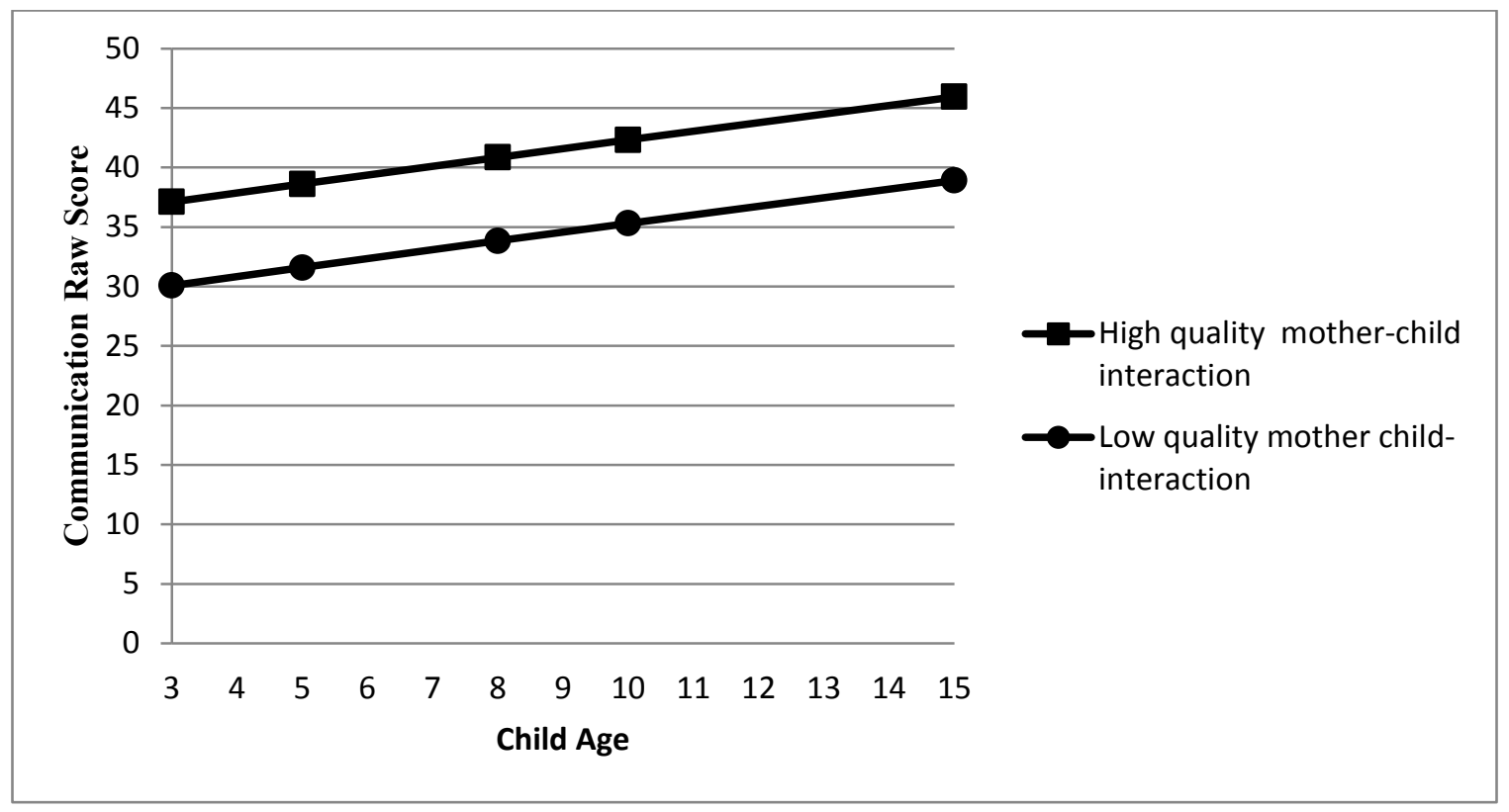

Figure 12. Negative life events as a predictor of communication scores for children with Down syndrome. This figure shows trajectories of communication scores for children with Down syndrome who have experienced numbers of negative life events equal to one standard deviation above and below the sample mean.

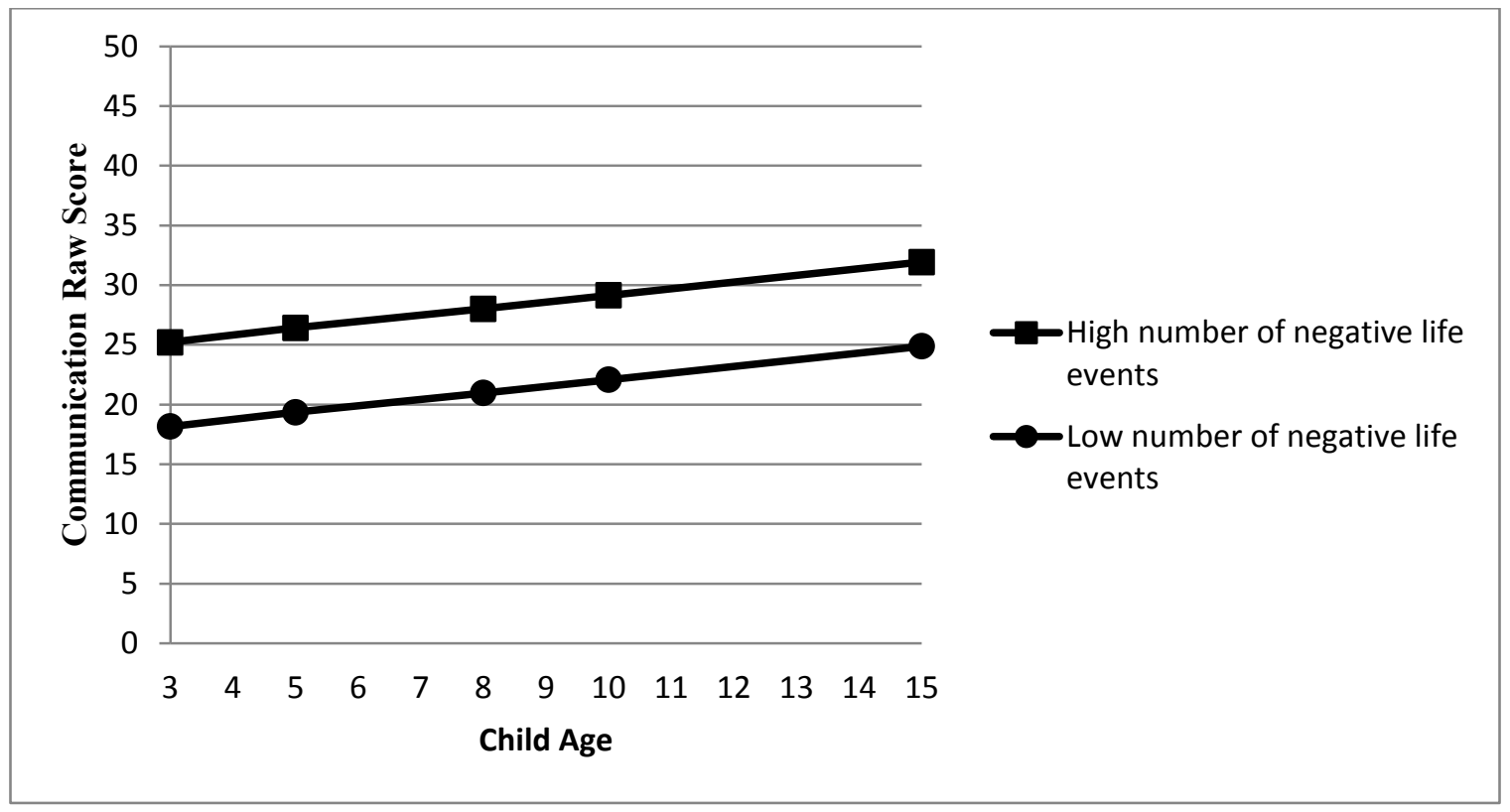


Figure 13. Negative life events as a predictor of communication scores for children with motor impairment. This figure shows trajectories of communication scores for children with motor impairment who have experienced numbers of negative life events equal to one standard deviation above and below the sample mean.

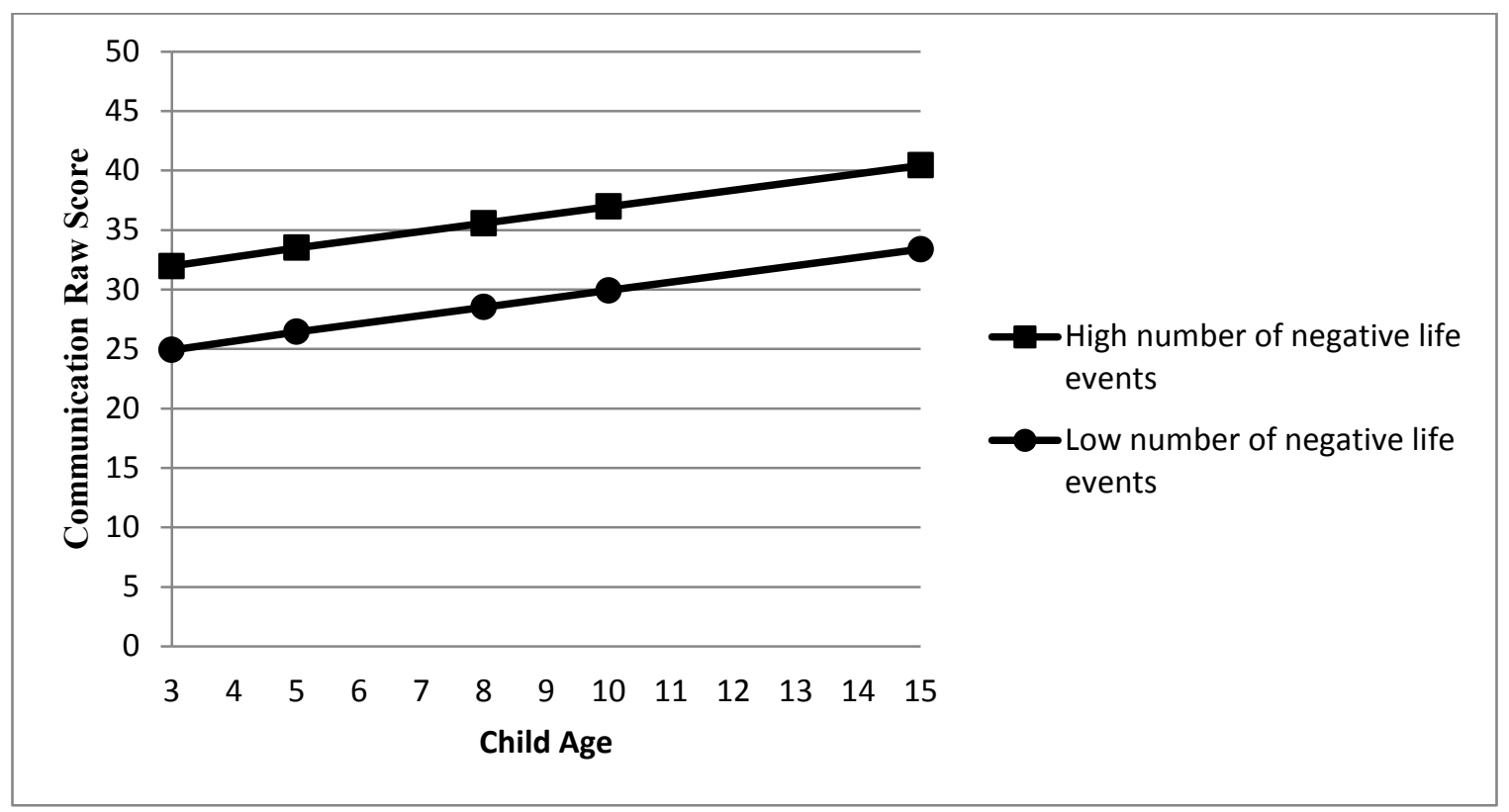

Figure 14. Negative life events as a predictor of communication scores for children with developmental delay of unknown etiology. This figure shows trajectories of communication scores for children with developmental delay of unknown etiology who have experienced numbers of negative life events equal to one standard deviation above and below the sample mean.

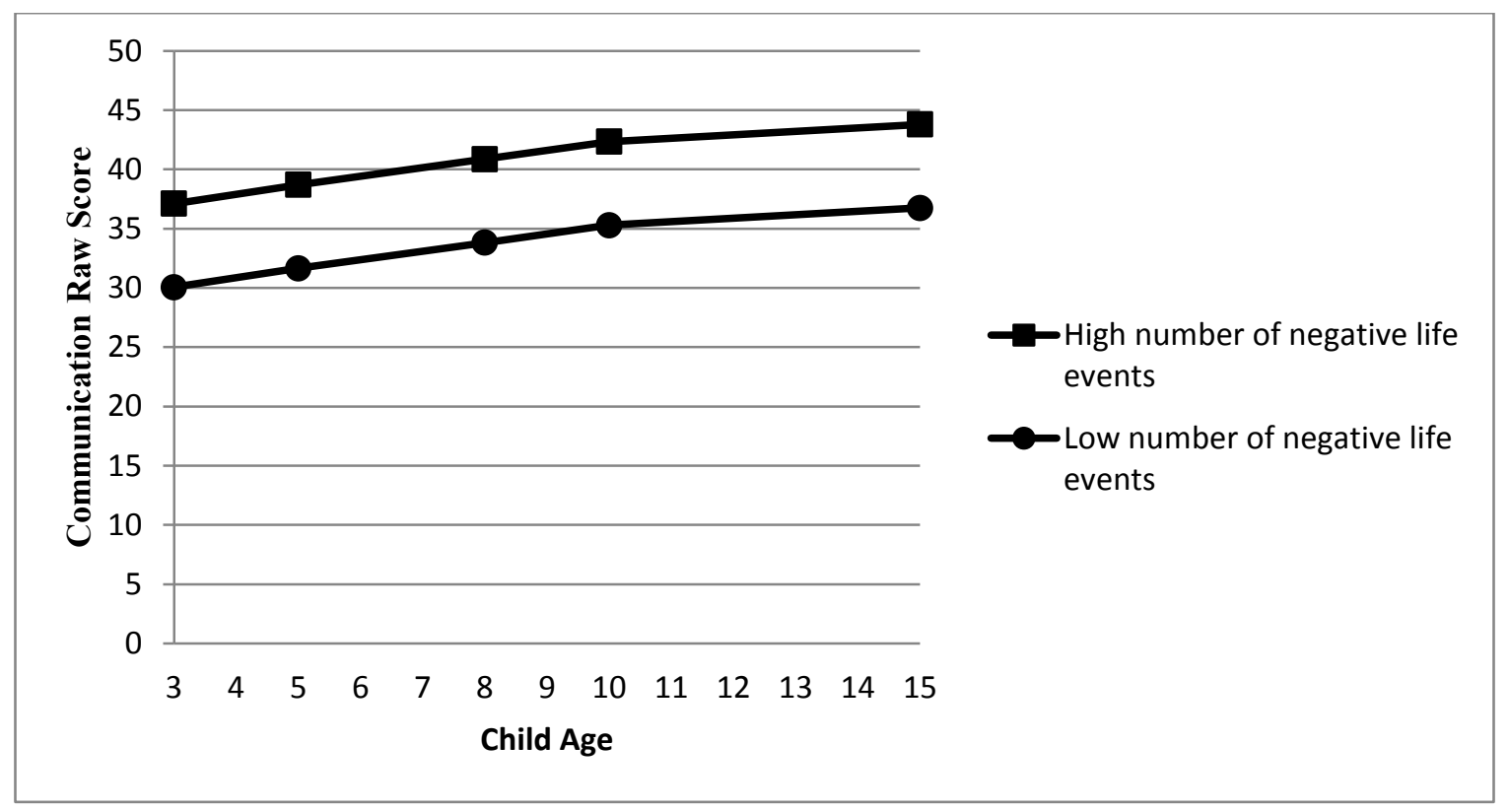


Figure 15. Mother-child interaction as a predictor of daily living scores for children with Down syndrome. This figure shows trajectories of daily living scores for children with Down syndrome who have mother-child interaction scores equal to one standard deviation above and below the sample mean.

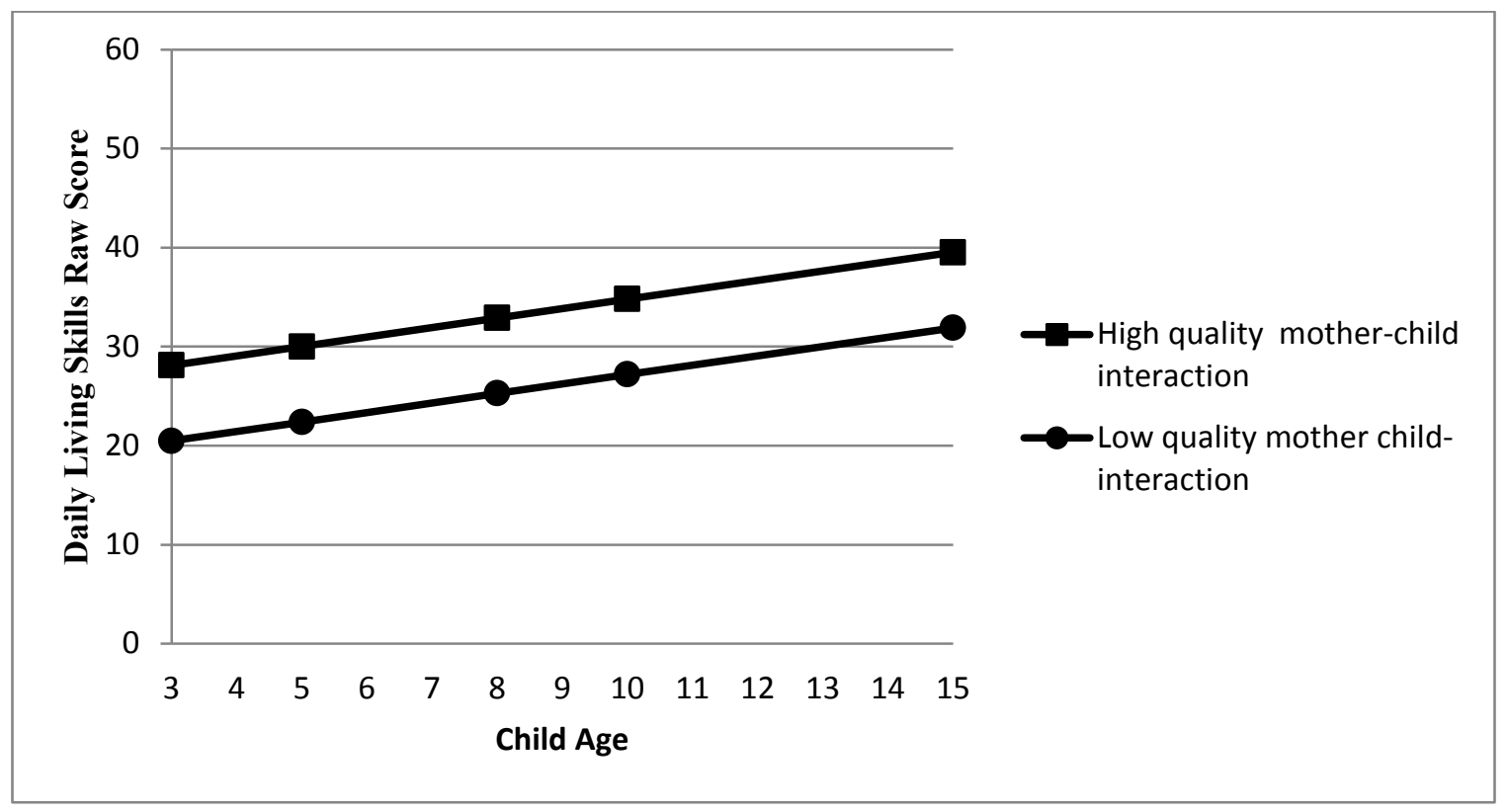

Figure 16. Mother-child interaction as a predictor of daily living scores for children with motor impairment. This figure shows trajectories of daily living scores for children with motor impairment who have mother-child interaction scores equal to one standard deviation above and below the sample mean.

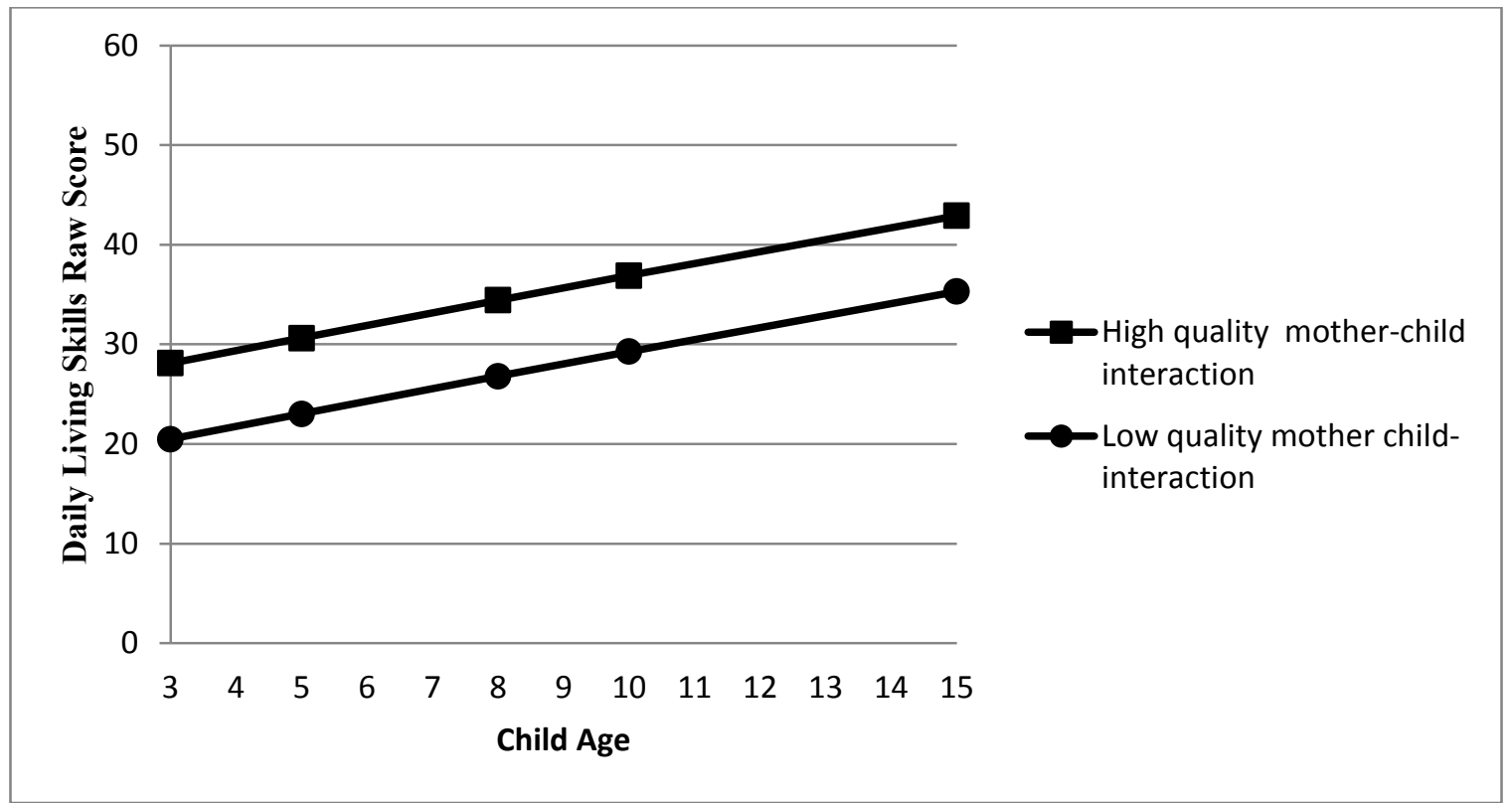


Figure 17. Mother-child interaction as a predictor of daily living scores for children with developmental delay of unknown etiology. This figure shows trajectories of daily living scores for children with developmental delay of unknown etiology who have motherchild interaction scores equal to one standard deviation above and below the sample mean.

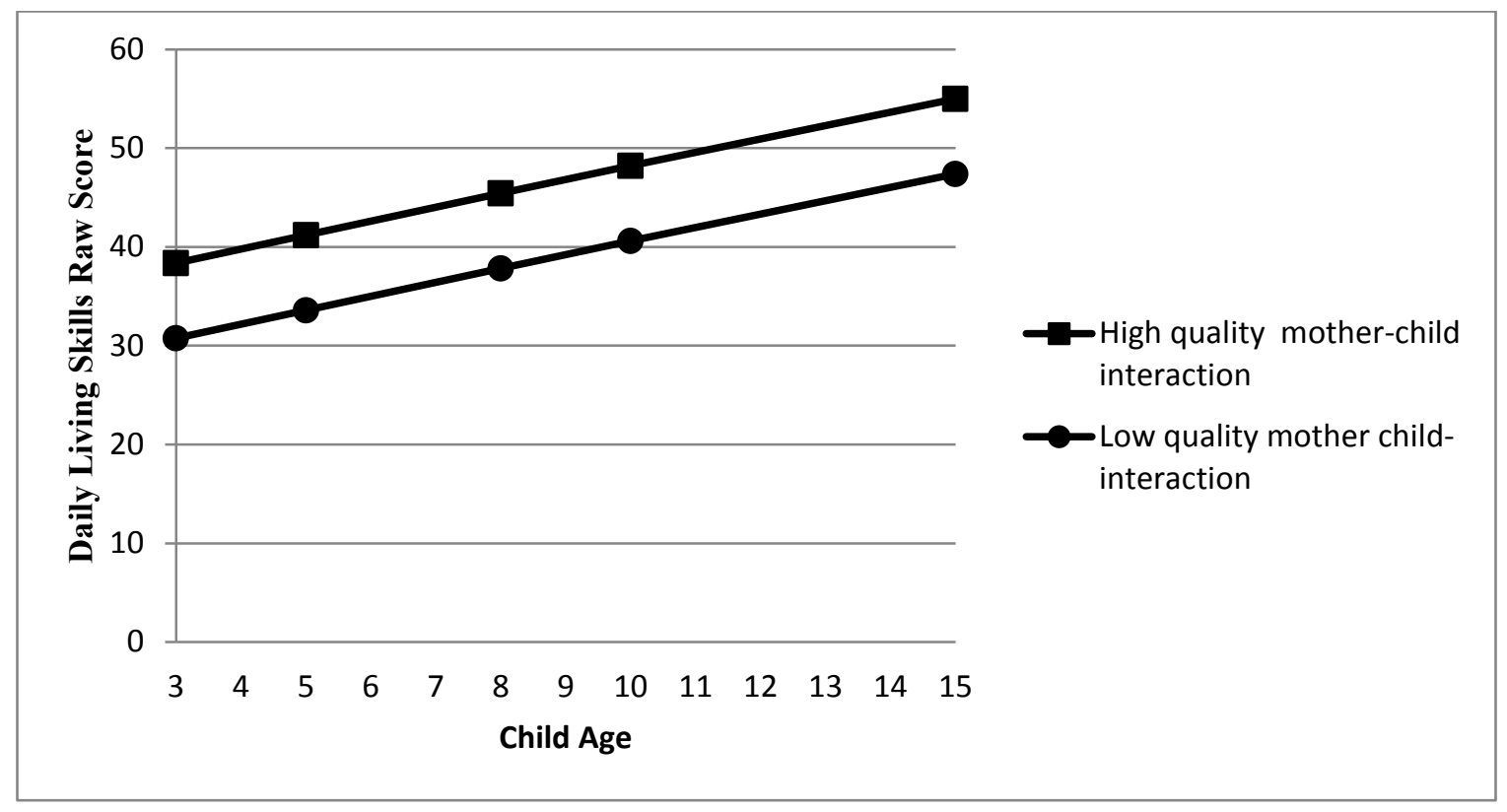

Figure 18. Negative life events as a predictor of daily living scores for children with Down syndrome. This figure shows trajectories of daily scores for children with Down syndrome who have experienced numbers of negative life events equal to one standard deviation above and below the sample mean.

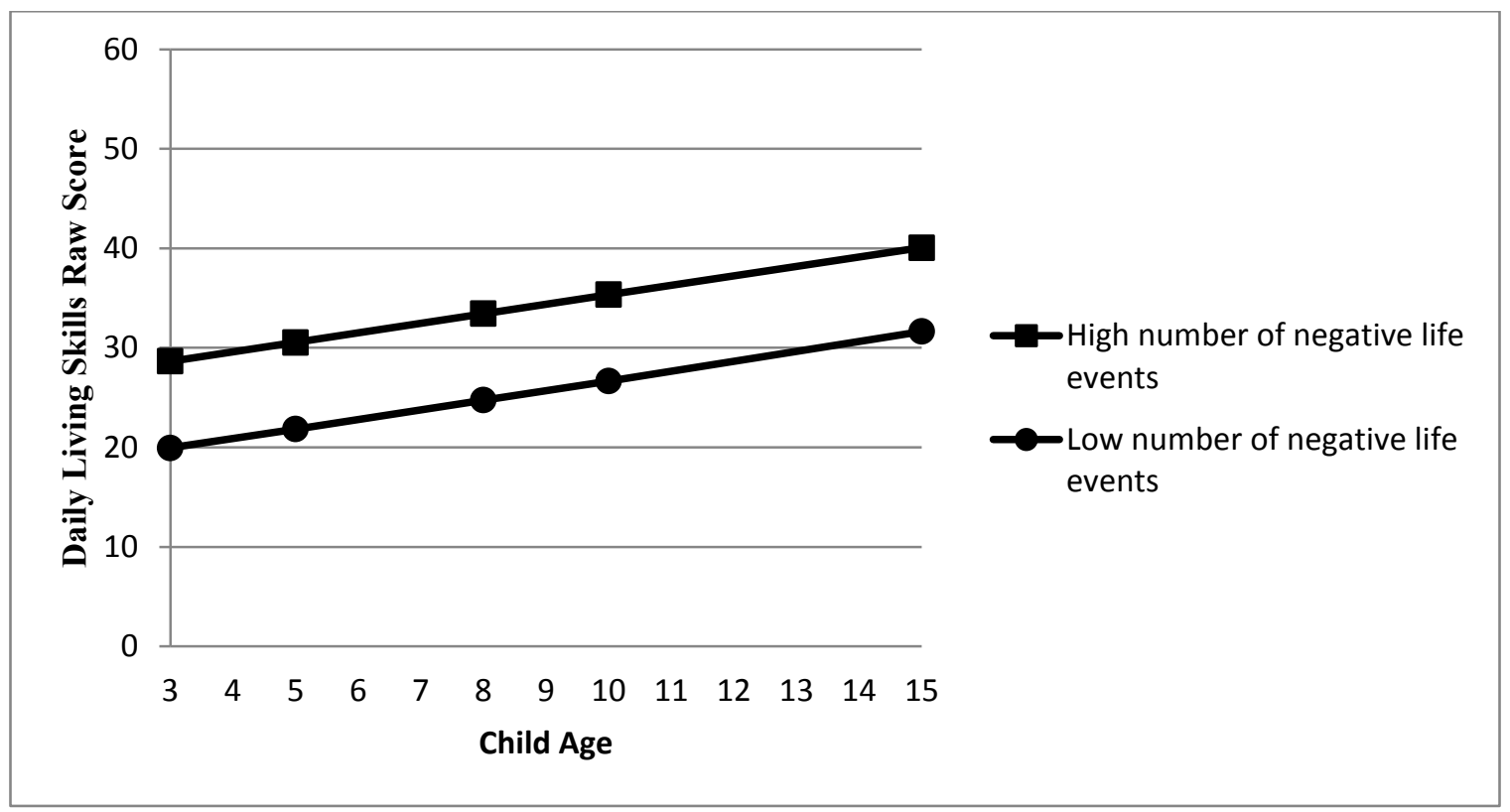


Figure 19. Negative life events as a predictor of daily living scores for children with motor impairment. This figure shows trajectories of daily scores for children with motor impairment who have experienced numbers of negative life events equal to one standard deviation above and below the sample mean.

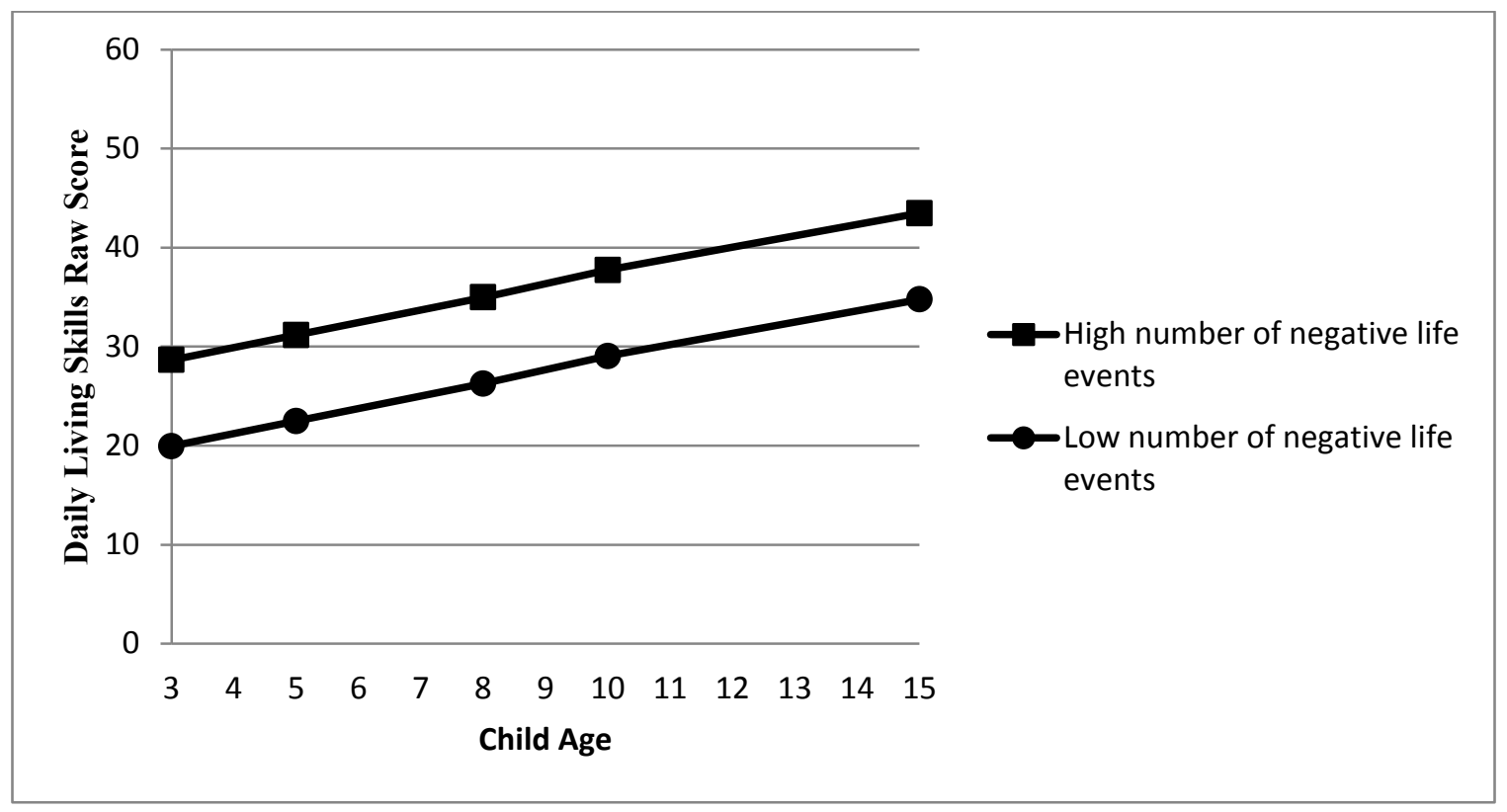

Figure 20. Negative life events as a predictor of daily living scores for children with developmental delay of unknown etiology. This figure shows trajectories of daily scores for children with developmental delay of unknown etiology who have experienced numbers of negative life events equal to one standard deviation above and below the sample mean.

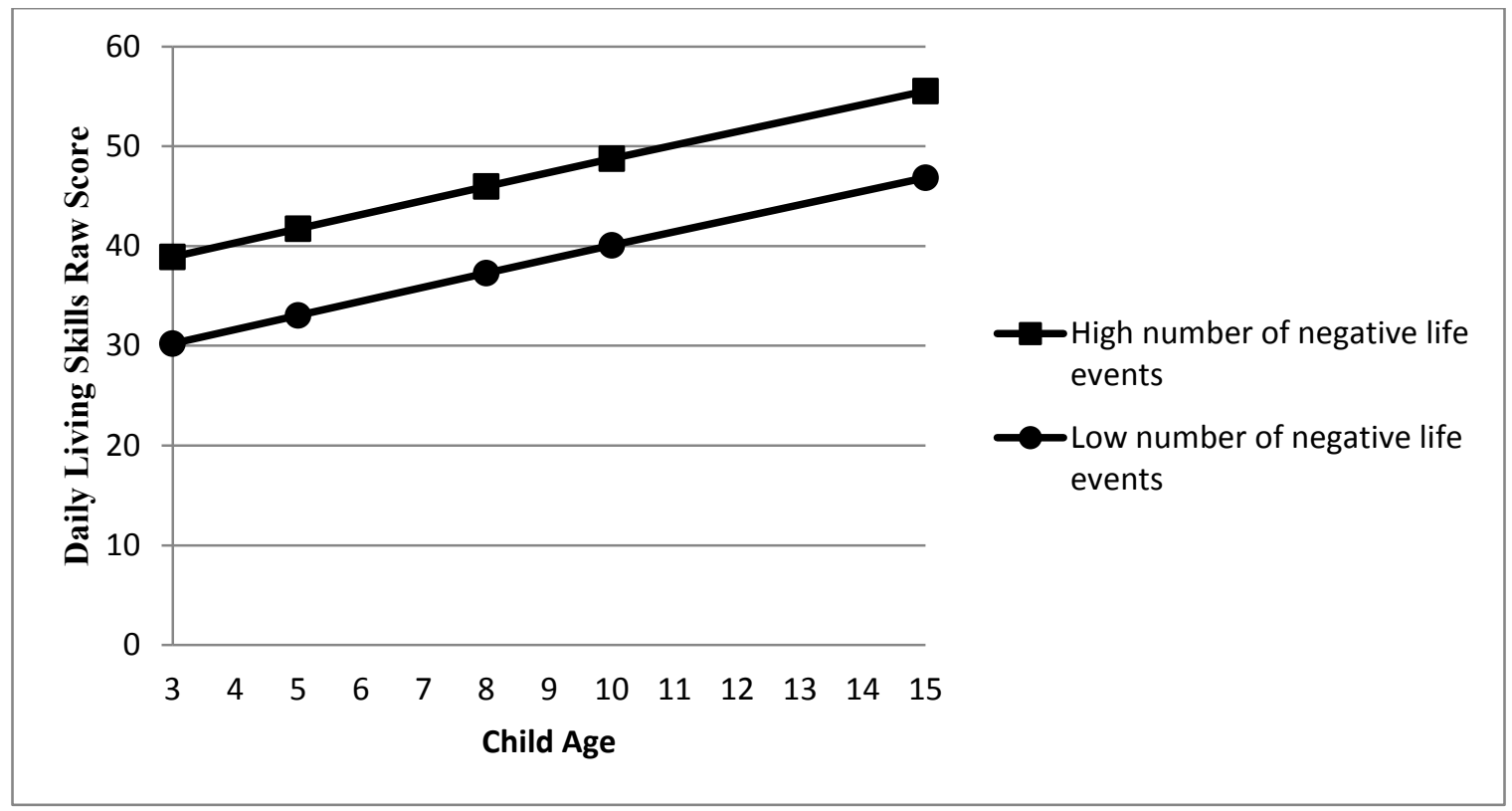


Figure 21. Mother-child interaction as a predictor of total adaptive functioning raw scores for children with Down syndrome. This figure shows trajectories of total adaptive functioning raw scores for children with Down syndrome who have mother-child interaction scores equal to one standard deviation above and below the sample mean.

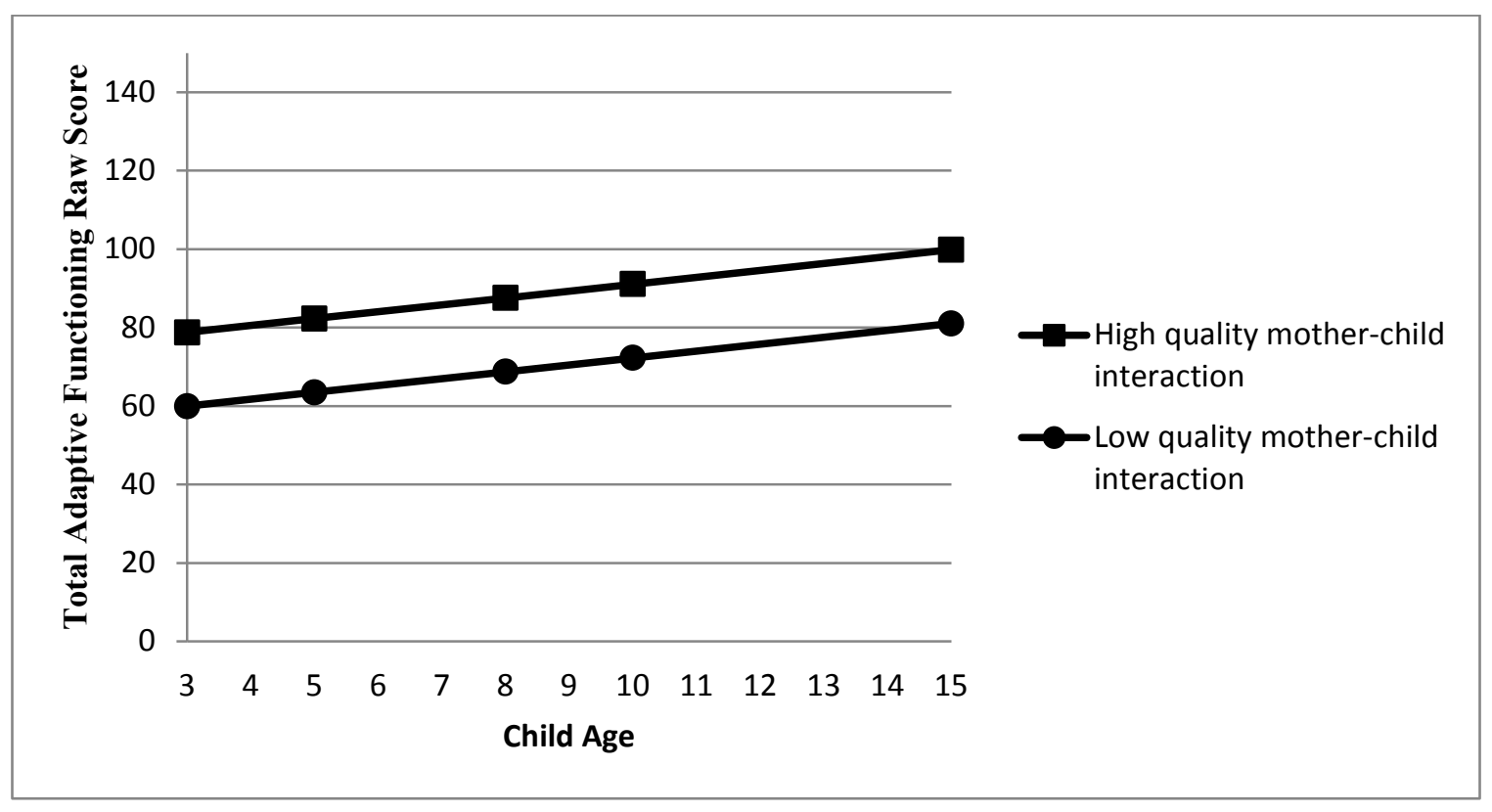

Figure 22. Mother-child interaction as a predictor of total adaptive functioning raw scores for children with motor impairment. This figure shows trajectories of total adaptive functioning raw scores for children with motor impairment who have motherchild interaction scores equal to one standard deviation above and below the sample mean.

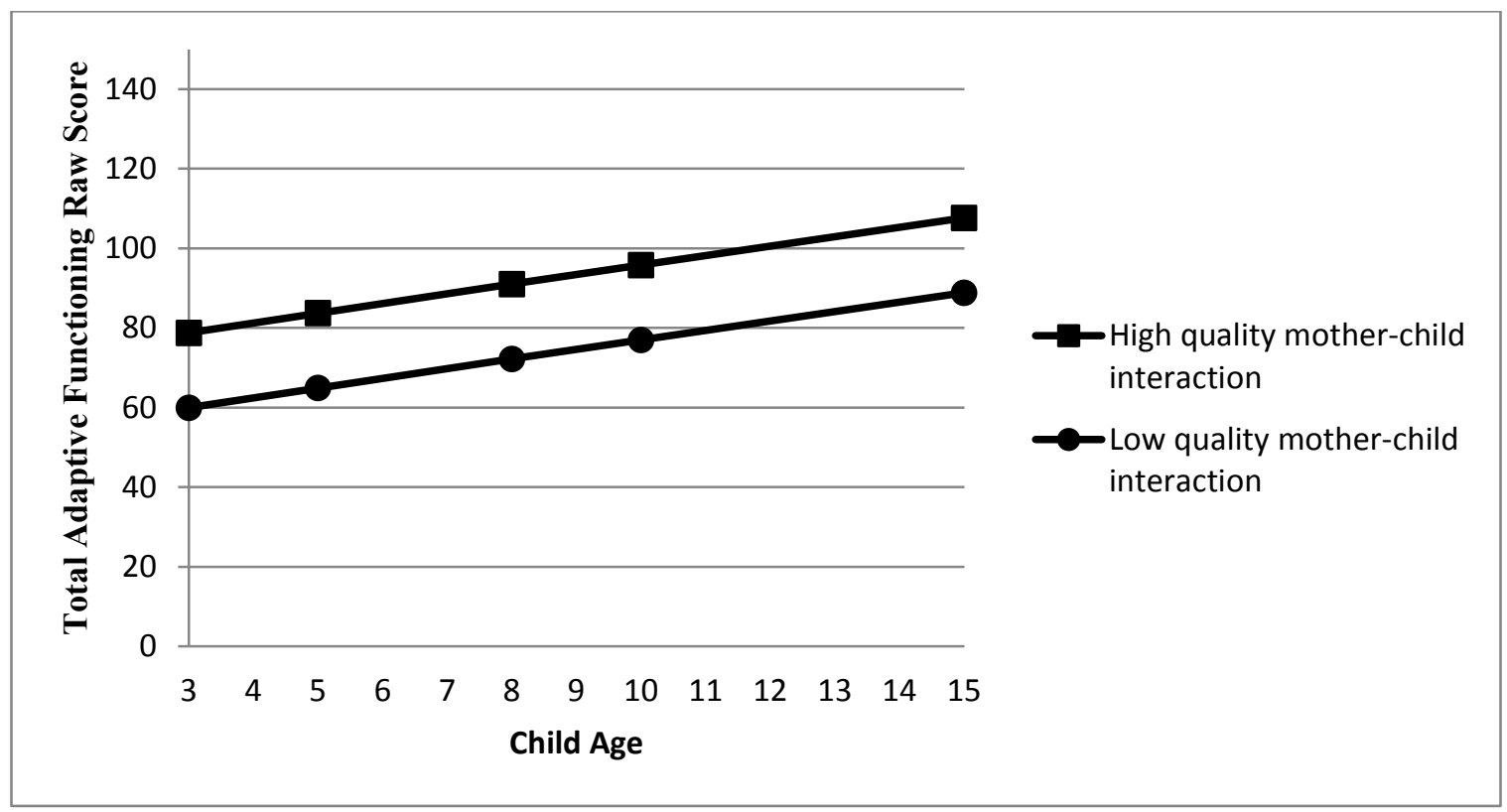


Figure 23. Mother-child interaction as a predictor of total adaptive functioning raw scores for children with developmental delay of unknown etiology. This figure shows trajectories of total adaptive functioning raw scores for children with developmental delay of unknown etiology who have mother-child interaction scores equal to one standard deviation above and below the sample mean.

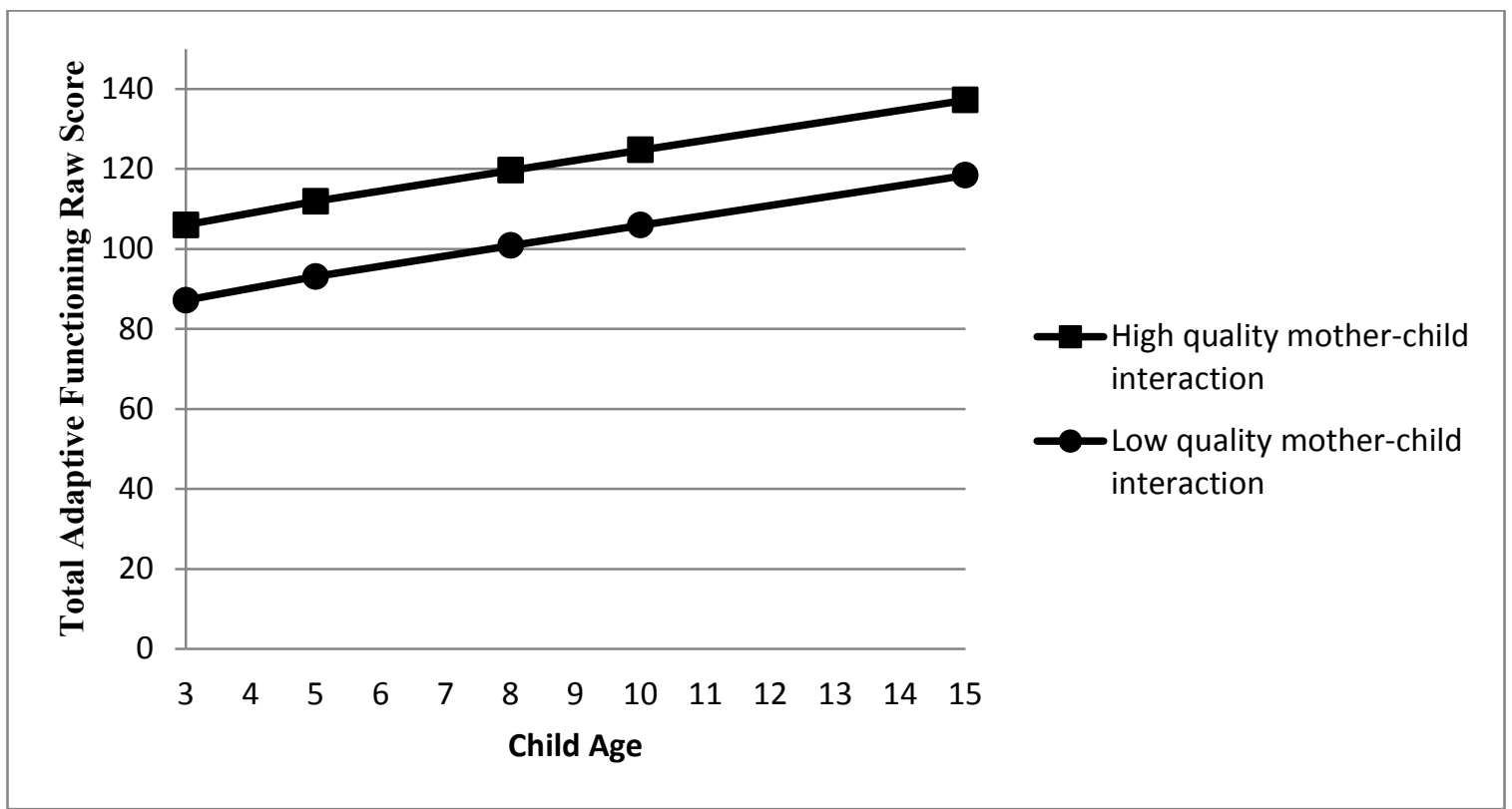

Figure 24. Negative life events as a predictor of total adaptive functioning raw scores for children with Down syndrome. This figure shows trajectories of total adaptive functioning raw scores for children with Down syndrome who have experienced numbers of negative life events equal to one standard deviation above and below the sample mean.

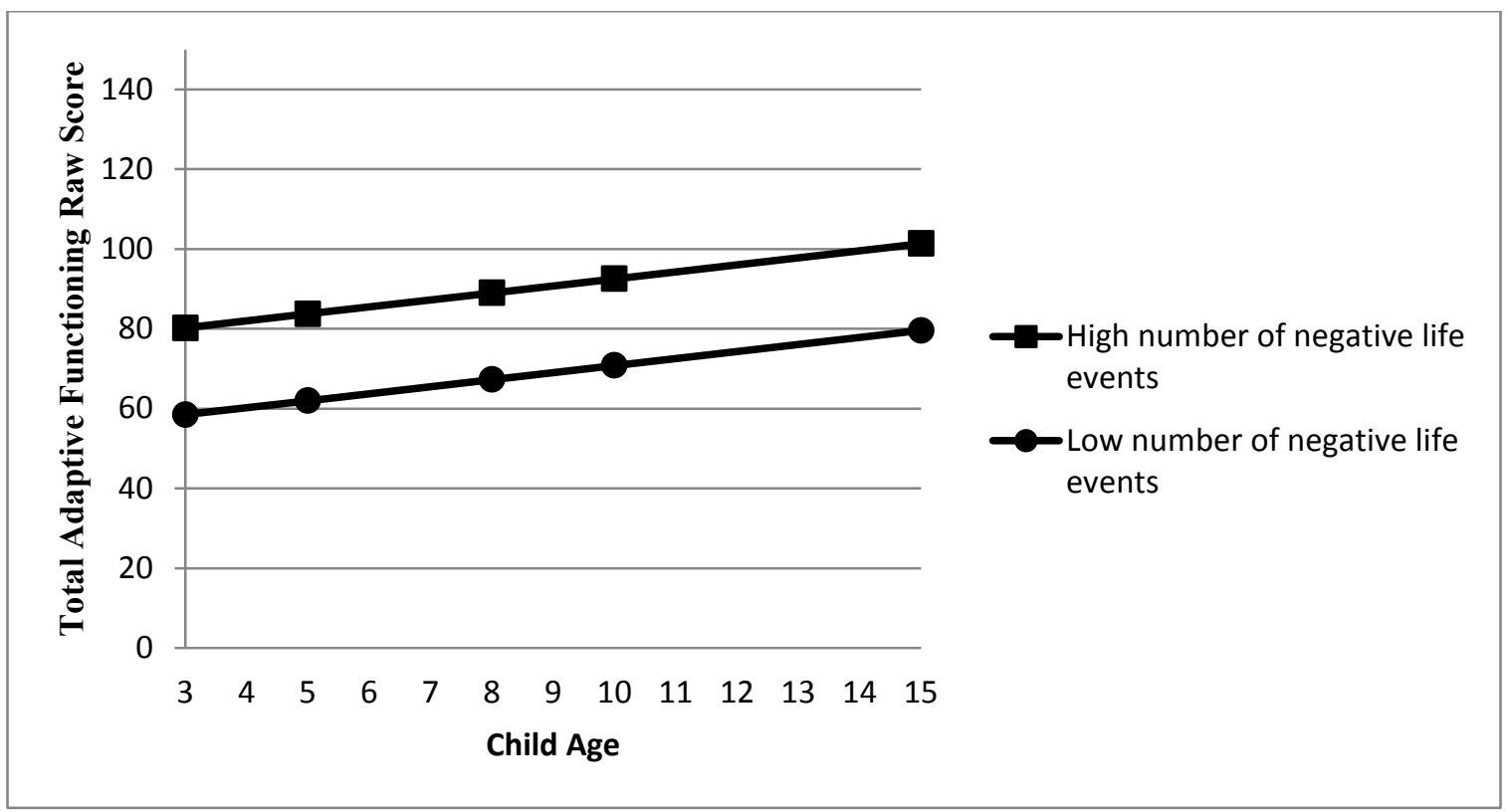


Figure 25. Negative life events as a predictor of total adaptive functioning raw scores for children with motor impairment. This figure shows trajectories of total adaptive functioning raw scores for children with motor impairment who have experienced numbers of negative life events equal to one standard deviation above and below the sample mean.

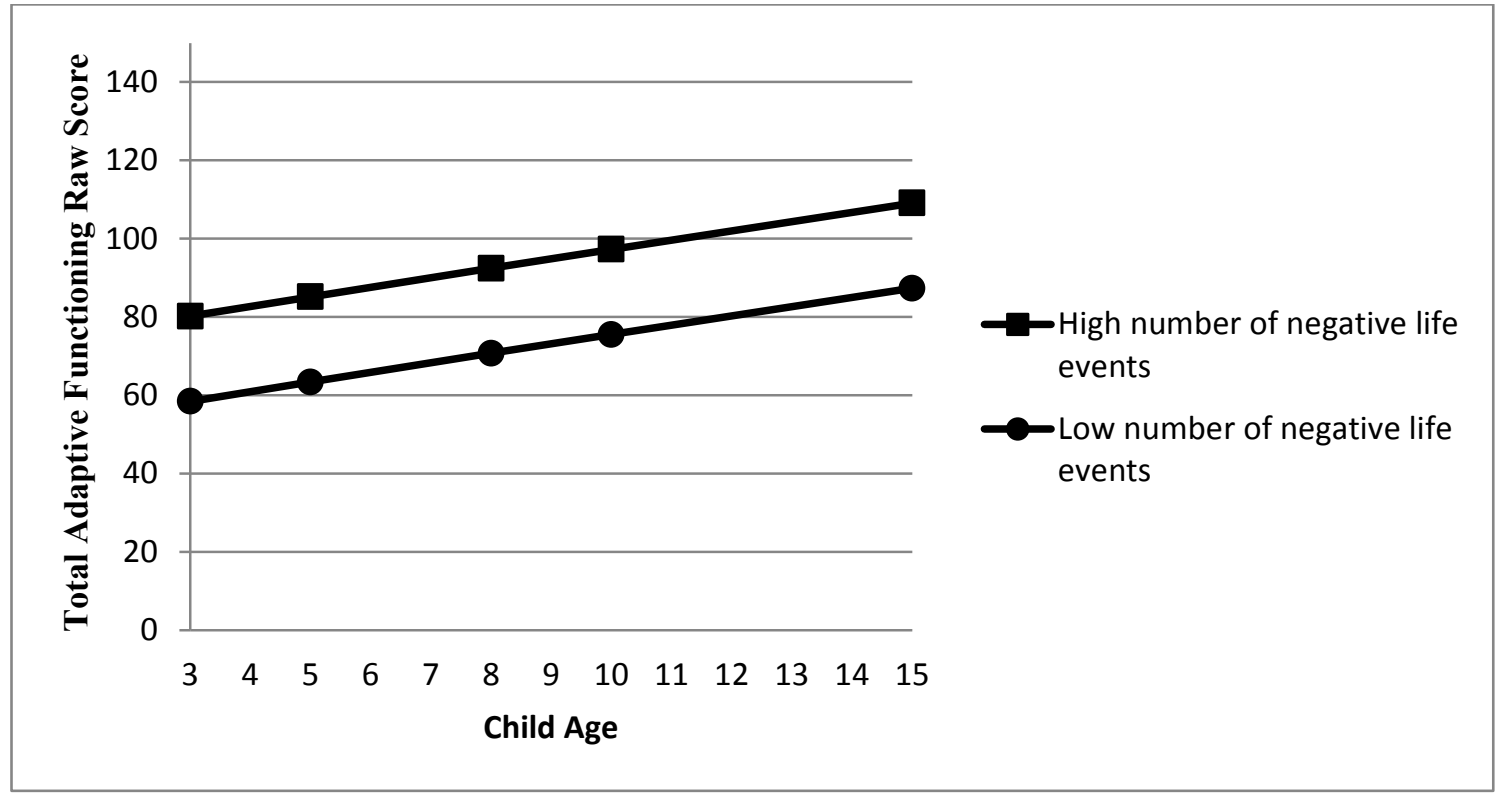

Figure 26. Negative life events as a predictor of total adaptive functioning raw scores for children with developmental delay of unknown etiology. This figure shows trajectories of total adaptive functioning raw scores for children with developmental delay of unknown etiology who have experienced numbers of negative life events equal to one standard deviation above and below the sample mean.

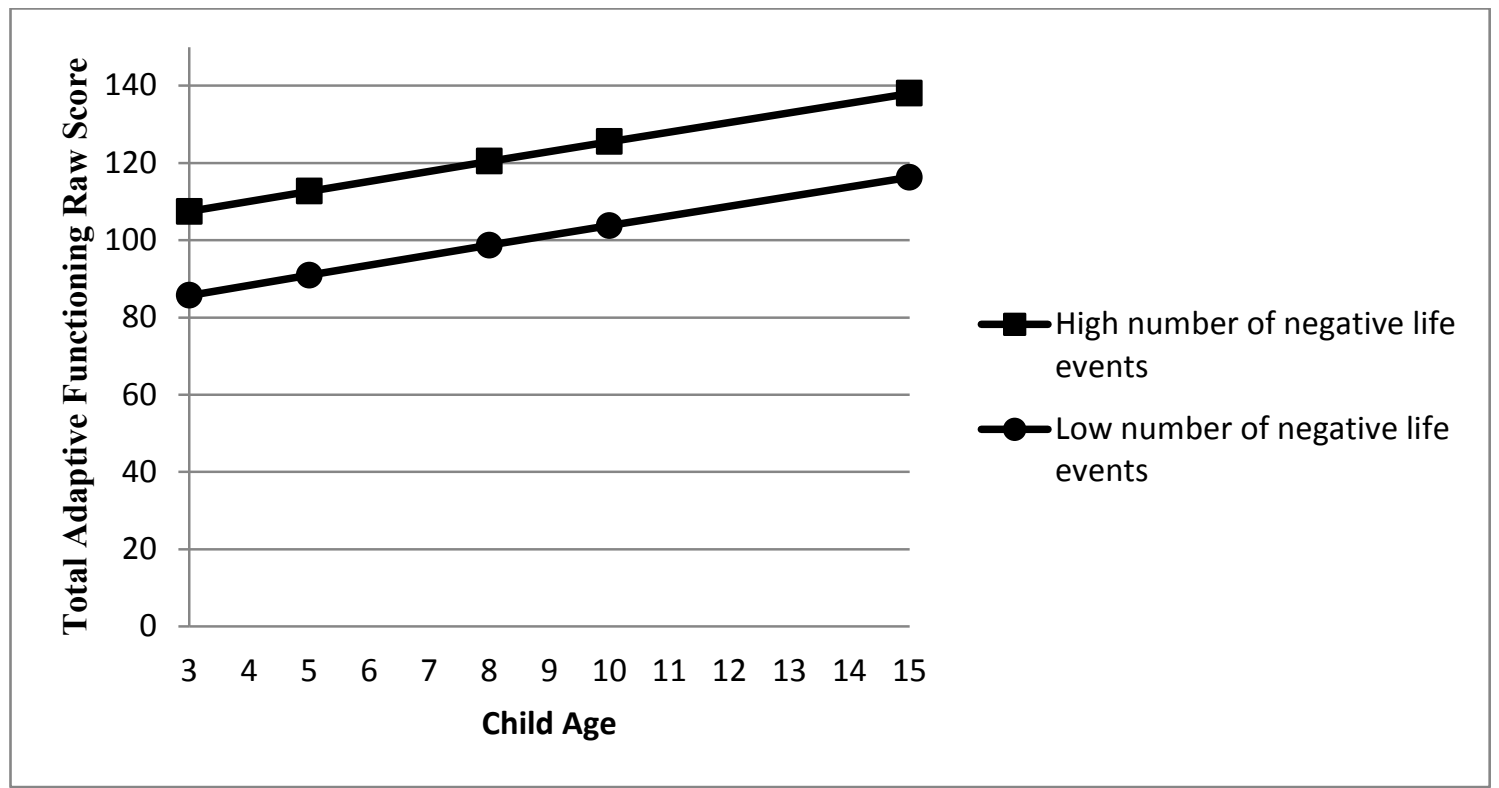

